\title{
Ginawaydaganuc and the Circle of All Nations: The Remarkable Environmental Legacy of Elder William Commanda
}

\author{
by \\ Romola Vasantha Thumbadoo
}

A thesis submitted to the Faculty of Graduate and Postdoctoral Affairs in partial fulfillment of the requirements for the degree of

Doctor of Philosophy

in

Geography

Carleton University

Ottawa, Ontario, Canada

(C) 2017

Romola Vasantha Thumbadoo 


\section{Abstract \\ Ginawaydaganuc and the Circle of All Nations: The Remarkable Environmental Legacy of Elder William Commanda}

This thesis is a case study analysis of the environmental work of the late 97 year old North American Indigenous elder William Commanda (11 November 1913 - 3 August 2011), a widely acknowledged public figure, guardian of three Algonquin wampum belts (sacred mnemonic record-keeping and governance devices including the Seven Fires Prophecy Belt, the Welcoming Belt, and Border Crossing Belt), Officer of the Order of Canada, and recipient of two honorary doctorate degrees.

William Commanda's work was grounded in the fully inclusive concept of Ginawaydagunuc that All is Related in the cosmic world. When the idea of We Are All Connected as people is extrapolated from this Ginawaydaganuc representation or metaphor, the word Anicinabe (Anishinabe in contemporary usage) assumes primacy as the good or real human being, and as such then includes Indigenous and non-Indigenous peoples. Those who align with his energetic web contribute to animate William Commanda's informal global eco-peace Circle of All Nations community.

I argue that the Circle of All Nations was conceptualized by William Commanda as a bridge-building mechanism and interface to speak to the incommensurability between Indigenous and non-Indigenous knowledge systems on environment, relationships and critical social justice and peace issues. The thesis incorporates and examines the pedagogical framework of this discourse.

The focus of the study is both abstract (whether and how his discourse impacts approaches to environment and related socio-ecological issues); and action oriented (regarding the continued relevance, animation and emergence of the discourse); and it employs a medicine 
wheel conceptual framework, and four logics of inquiry: performance mapping, historical dialectical, phenomenological and cybercartographic.

It explores how narrative, geo-narrative, critical reflexivity and graphic, cybercartographic geo-narrative, digital atlas and social media methodological tools can be justified and employed to support knowledge generation on environment, relationships and related matters in contemporary times. It also explores how such research and scholarship might impact social change and environmental studies. 


\section{Acknowledgements}

In our book, Learning from a Kindergarten Dropout: William Commanda - Ojigkwanong, Cultural Sharings and Reflections, by Romola V asantha Thumbadoo, we wrote "Romola and I are happy to share this book with you. We dedicate it to the spirit of the ancestors who have guided our efforts, to the incredible circle of people who have enriched our lives, and to the children of the future who shall create a culture of peace". Now I am happy to share his thesis in the academic world, and reiterate the same message.

My journey with William and his Indigenous story began twenty years ago, and it has been an incredible learning and growing experience. It has taught me so much personally and professionally. The journey has not been without sacrifice, particularly to my mother, and my family in South Africa, and my family in Canada and the United States. I acknowledge this with tears, humility and deep gratitude. I come from a family of teachers, and this thesis enables me to honour that heritage now. My sisters have each played a role in my studies. When I was struggling to write my thesis proposal, my librarian sister Camilla put the whole framework in order over the course of a telephone conversation. Zeni's passionate Isibindi child and youth care work, which Elder Commanda himself blessed in South Africa, has inspired my studies and Carleton University has enabled me to integrate common themes in my academic thinking and Circle of All Nations outreach. Beulah, a social entrepreneur, literacy, environmental and animal rights activist, introduced me to Aldo Leopold, and this eventually brought me to the Department of Geography and Environmental Studies. I am grateful for what I have learned from William Commanda's family and community, and in particular, I thank his daughter, Evelyn Commanda Dewache, sister Mary Commanda, and nieces Mary Daisy Commanda Jacko and Lillian Dowling for all they have shared with me over the years. I thank our incredible Circle of All Nations 
community for giving life to William Commanda's teachings and outreach, and for their ongoing efforts to animate his work and their inspiration during the years of challenge since William's passing. They constitute the heart and hope of this thesis, and I see his seeds sprouting everywhere. Dean, Ann, Fred, Paula, Karen, Sunbow, Carolyn and Rob have helped me grapple with many ideas over the years, and have played unique roles in shaping and strengthening my thinking.

I am most grateful for the support of the Department of Geography and Environmental Studies, especially the guidance from Gita Ljubicic, and of the Geomatics and Cartographic Research Centre team, including Stephanie Pyne. Professor Scott Simon and the University of Ottawa have helped to introduce William Commanda to the local and international academic world. My Indigenous thesis committee members Douglas J. Cardinal, Architect, and Simon Brascoupe, Algonquin Artist and Professor, have both played very important roles to help me share William's messages with courage.

It has been a challenge returning to the academy as an adult, after years of entrenchment in bureaucracy, and then grass roots work. I know that I cannot express my gratitude to my supervisor, Fraser Taylor, adequately. William and I met him ten years ago, and we both knew that we wanted to share Elder Commanda's land-based story on the innovative digital atlas platform that Professor D. R. Fraser Taylor was pioneering. Dr. Taylor's passion, focus and drive are inspirational. He has taken me through every step of my academic journey tirelessly, and he has enabled me to present William Commanda's teachings with integrity, to build bridges in the world of spiraling cybernetic loops, and ensure that he remains Encore Vivant! I have learned so much over these past five years at Carleton University, and am strengthened for the journey ahead.

\section{Megwetch! Merci! Ginawaydaganuc!}




\section{Contents}

Title Page $\quad$ i

Abstract

Acknowledgements iv

Table of Contents vi

$\begin{array}{ll}\text { List of Figures } & \text { ix }\end{array}$

Chapter 1

Introduction $\quad 1$

1.1 William Commanda and his Academic Interests 1

1.2 William Commanda's Background 3

1.3 A Mini-Biography 3

1.4 Guardian of Sacred Wampum Heritage 5

$\begin{array}{lll}1.5 & \text { Situating Myself } & 13\end{array}$

1.6 The Thesis Presentation 14

\section{Chapter 2}

Theory

2.1 Theoretical Framework 16

2.1.1 Locating the Research in Geography, Cartography and

Cybercartography with Linkage to Environment 16

2.1.2 The Research Question 16

2.1.3 Circle of All Nations Logo as Theoretical Frame 19

2.1.4 Thematic Context 21

2.1.5 The Logics of Inquiry 22

2.1.6 Discourse Analysis Approach 24

2.1.7 Case Study 26

2.2 Structural Parameters 29

2.2.1 Geography, Cartography, Environment and Mother Earth 29

2.2.2 The Foundational Concept: Ginawaydaganuc All is Related 32

2.2.3 Syntropy - An Emerging Concept to complement an Ancient One, Ginawaydaganuc 34

$\begin{array}{lll}2.2 .4 & \text { Pentimento } & 37\end{array}$

$\begin{array}{lll}2.2 .5 & \text { Storytelling } & 38\end{array}$ 


\section{Chapter 3}

3.1 Overview 43

3.1.1 The Undergrowth 43

3.1.2 The Methodological Approach 47

3.2 The Four Logics of Inquiry 51

3.2.1 Conceptual Medicine Wheel Orientation Map 52

3.2.2 Performance Mapping, and the Geo-narrative Ideological Foundation 52

3.2.3 Historical/Dialectical Approach and Narrative Exploration 53

3.2.4 Phenomenology, and Auto-Ethnography and Critical Reflexivity 54

3.2.5 Cybercartography, and the Cybercartographic Digital Atlas and
Social Media

3.3 Data Analysis $\quad 57$

\section{Chapter 4}

Theoretical Lens: Performance Mapping and Geo-Narrative Mamiwinini Trail Marking - and Syntropic Agency of the Seven Fires Prophecy Wampum Belt

4.1 Orientation into the Performance Mapping Logic of Inquiry Lens 59

4.2 Theoretical Frame, Methodology and Tools: Performance Mapping Geo-narrative 63

4.3 Conceptual Medicine Wheel Orientation Map 75

4.4 Discussion, Analysis and Observations 75

4.4.1 Special Rock Features of the Ottawa River Valley and Algonquin Heritage 75

4.4.2 Language, Linguistics, Semantics, Semiotics and Performative Technologies 84

4.4.3 Algonquin Ideology and Knowledge Generating Practices as Embedded in
Rock Art and Animated in Wampum Agency

4.4.4 Spirit Energy of the Land 104

4.5 Concluding Remarks 111

4.6 Summary of Findings 115

4.7 A William Commanda Mapping Exemplar 117

\section{Chapter 5}

Theoretical Lens: Historical Dialectical Logic of Inquiry The Quebec Welcome Kwe Beckian! Hello Stranger! in accordance with the integrity of the Three Figure Welcoming Wampum Belt

5.1 Orientation into the Historical Dialectical Logic of Inquiry Lens 119

5.2 Theoretical Frame, Methodology and Tools:

Historical/Dialectical 129

$\begin{array}{ll}\text { 5.3 Conceptual Medicine Wheel Orientation Map } & 137\end{array}$

$\begin{array}{lll}5.4 & \text { Discussion, Analysis and Observations } & 138\end{array}$

5.4.1 The First Narrative - Maudit sauvage 138

5.4.2 Second Narrative - Indigenous Visibility and Leadership Presence 152

5.4.3 Third Narrative - A William Commanda Lament 155

5.4.4 Fourth Narrative - A Sampling of William Commanda's Teaching Tools 162 
5.5 Concluding Remarks 181

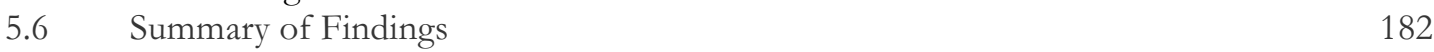

5.7 A William Commanda Historical Narrative Exemplar 184

\section{Chapter 6}

Theoretical Lens: Phenomenological Logic of Inquiry Seeding Negentropy and Becoming Good Enough for Two The Border Crossing Wampum Belt and the Manashkadosh Exemplar

6.1 Orientation into the Phenomenological Logic of Inquiry Lens

6.2 Theoretical Frame, Methodology and Tools: Phenomenology, Auto-ethnology and Reflexivity

6.3 Conceptual Medicine Wheel Orientation Map 205

6.4 Discussion, Analysis and Observations 205

6.4.1 Artistic Expression, Creativity and Reflexivity 205

6.4.2 Beyond Borders 214

6.4.3 Boreal Rendezvous 218

6.4.4 Canoe as Epistemological, Ontological and Ontogenetic Tool 225

6.5 Concluding Remarks 230

6.6 Summary of Findings 231

6.7 A William Commanda Phenomenological Exemplar 232

\section{Chapter 7}

\section{Theoretical Lens: Cybercartographic Geo-Narrative} Encore Vivant and Cyber Steering - Potential and Volition inspired by the Missing

7.1 Orientation into the Cybercartographic Geo-Narrative Logic of Inquiry Lens

7.2 Theoretical Frame, Methodology and Tools: Cybercartography, Auto-Ethnography and Critical Reflexivity

7.3 Conceptual Medicine Wheel Orientation Map 245

7.4 Discussion, Analysis and Observations 246

$\begin{array}{lll}\text { 7.4.1 Cybernetics } & 246\end{array}$

$\begin{array}{lll}\text { 7.4.2 Agency of Animals } & 249\end{array}$

7.4.3 Asinabka Eco-Peace Think Tank 258

7.4.4 Contemporary Work Generated as William Commanda’s Legacy 262

7.5 Concluding Remarks 265

7.6 Summary of Findings 266

7.7 A William Commanda Cyber Exemplar $\quad 272$

\section{Chapter 8}

Conclusion 


\section{List of Figures}

F.1 William Commanda receiving his Honorary Doctorate Degree at the University of Ottawa's October 2006 Convocation, and filing application for doctoral studies 2009

F.2 Circle of All Nations Logo

F.3 Conceptual Map of Circle of All Nations Logics of Inquiry

F.4 Conceptual map of Circle of All Nations Performance MappingGeonarrative Lens into Thesis

F.5 Conceptual Map of Circle of All Nations Historical / Dialectical Lens into Thesis

F.6 Circle of All Nations Conceptual Medicine Wheel (1a)

F.7 Examples of Circle of All Nations use of the Conceptual Medicine Wheel (1b)

$\begin{array}{lll}\text { F.8 The Frog CAN Pedagogical Tool } & 172\end{array}$

$\begin{array}{lll}\text { F.9 The Foundations Transparency } & 173\end{array}$

$\begin{array}{lll}\text { F.10 The Results Transparency } & 174\end{array}$

$\begin{array}{lll}\text { F.11 The Opportunities Transparency } & 175\end{array}$

F.12 The Goals Transparency 176

$\begin{array}{lll}\text { F.13 Layering of Transparencies and Issues } & 177\end{array}$

F.14 Superimposition of Law of Nature Energy and Values 178

$\begin{array}{ll}\text { F.15 Ideological Differences } & 179\end{array}$

F.16 Conceptual Map of Circle of All Nations Phenomenological, Auto-Ethnographic Reflexive Lens into Thesis 205

F.17 Good Enough for Two Documentary 231

F.18 Conceptual Map of Circle of All Nations Cybercartographic Lens into Thesis 245

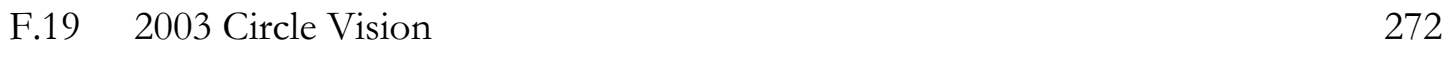


F.20 2007 Asinabka Website 273

F.21 Grandfather's Prayer at the Chaudiere 273

F.22 Asinabka National Indigenous Centre Brochure (English) 274

F.23 Asinabka National Indigenous Centre Brochure (French) 274

F.24 Asinabka National Indigenous Centre Brochure - Details (English) 275

F.25 Asinabka National Indigenous Centre Brochure - Details (French) 275

F.26 William Commanda Collage 276

F. 27 Artistic Depiction of William Commanda at Chaudiere 277 


\section{Chapter 1 Introduction}

\subsection{William Commanda and his Academic Interests}

The thesis examines the discourse and legacy of late Indigenous elder, William Commanda. It focuses on his approach to Mother Earth, environment and relational matters. For forty years, he was the carrier of three Algonquin wampum belts, critically important sacred and historical mnemonic teaching and record keeping devices, and was the longest known guardian of such an Indigenous heritage and responsibility. He was awarded two doctoral degrees, one (doctor of philosophy) by the University of Ottawa in 2006, and the other (doctorate honorifique causa) by l'université du Québec-Outaouais in 2011. He was appointed Officer of the Order of Canada in 2008, this country's penultimate honour (Desiderantes Meliorem Patriam). Carleton University named the Aboriginal Resources Centre Ojigkwanong after him in 2013, and the University of Ottawa created the Dr. William Commanda Hall in 2014, in acknowledgment of his contributions to the academic world.

William Commanda's Circle of All Nations work was initiated with a gathering of Indigenous and non-Indigenous peoples from across North America in 1967, the year of Canada's $100^{\text {th }}$ birthday. He sought to integrate Indigenous thinking, environmentalism, activism, social justice and peace building, in order to both challenge agendas of colonial domination and environmental degradation, and also advance dialogue, reconciliation, healing and social change engagement amongst diverse players. William Commanda remains very much present in cyberspace to this day, six years after his death. Multiple diverse voices from Canada and abroad demonstrate ongoing commitment to ensuring that he remains encore vivant, through their inclusion of him in considerations of critical issues of the day, in academic research, activism and social media communications. 
In his formal acceptance of the Honorary Doctorate Degree at the October 30, 2005

University of Ottawa Convocation, William Commanda said:

Today, I become a doctor - but really, I don't know if I am that smart - I have been suffering with shingles for the past three weeks, and I still have not been able to cure myself!

But despite the pain, I am very happy to be here with you.

I accept this honour with great gratitude, and really, I accept it on behalf of my Indigenous ancestors and children. For a long time we have been ashamed of our heritage; there were dark moments when I myself felt it was a shame to be born Indian and wished I were dead. But a deeper strength of my ancestors kept me alive, and taught me to work for the two important things they believed in:

First, absolute respect for Mother Earth and the Waters, Air and Fire, and the Animal Teachers. Today, we see where our collective disrespect for Mother Earth has brought us: to the destruction of life as we have known it. Today we worry daily about natural disasters, cancers and new diseases.

My ancestors also taught that we were all related, and so we learned to treat everyone and everything on the Earth with respect, generosity and love: as a relative. As we moved away from those values, exploitation, hatred, violence and crime poisoned our lives and destroyed our world.

As I learned to find my path back to the values of my ancestors, so I gradually built my world around the vision of a Circle of All Nations, a Culture of Peace.

I am deeply moved that the University of Ottawa recognizes this effort with this honorary degree to a kindergarten dropout!

I hope it encourages Indigenous Peoples to recognize and realize the strengths and wisdom within our heritage; and that it also encourages others to learn and value the teachings of Turtle Island, the place they all now call home.

I thank my many friends who have travelled here to support me today. It is their support that has made this honour possible.

Megwetch, Merci (William Commanda Personal Archives)

Years later, this is how he viewed his convocation: "They brought me to the University of Ottawa and they dressed me up and made me a doctor - just because I work with all colours, all races! Kindergarten Dropout, that's me!” (Personal Conversation 2010). It was his own recognition of the importance of the Indigenous voice to Canada's 
increasingly diverse population.
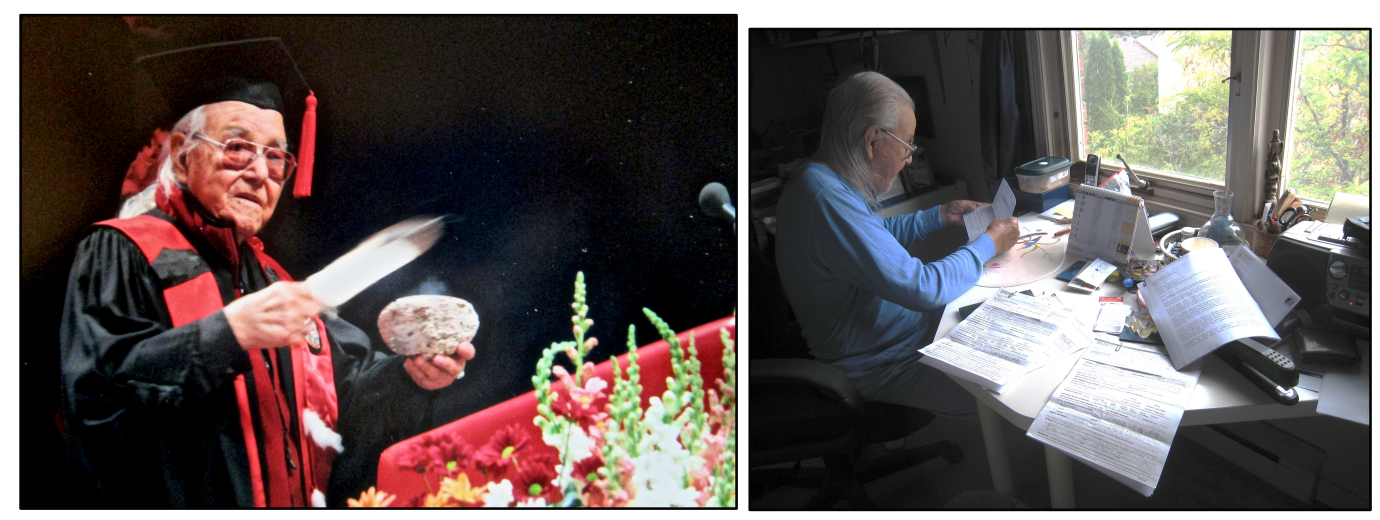

Figure 1. William Commanda receiving his Honorary Doctorate Degree at the University of Ottawa's October 2006 Convocation (left), and filing application for doctoral studies in 2009 (right)

The following photographs capture the 1 October 2009 applications for admission to university. Note that we filed individual applications and paid our own application fees.

\subsection{William Commanda's Background}

The late Indigenous elder, William Commanda, carrier of sacred Algonquin wampum belts, and founder of the Circle of All Nations is a widely acknowledged public figure. His lifetime spanned the last century (11 November 1913 - 3 August 2011). He engaged actively with others to impact and contribute to the larger world, particularly with respect to Mother Earth, peace and environmental issues.

\subsection{A Mini-Biography}

The following is his mini-biography from the Circle of All Nations website:

A Mini-Biography of Dr. William Commanda, OC, Algonquin Elder, Founder of A Circle of All Nations

11-11-1913 to 03-08-2011

Algonquin Elder William Commanda from Kitigan Zibi Anishinabeg, Maniwaki, Quebec was born on November 11, 1913 under the bright light of the Morning Star, so his mother named him Ojigkwanong; thus the larger universe figured in his 
personal story from the very beginning. Today, he is seen by many as the symbol of light emerging from the darkness of the First World War, illuminating a path to a new world with his vision for a Circle of All Nations, A Culture of Peace.

He was a respected spokesman and spiritual leader at many conferences, participated in United Nations peace and spiritual vigils, and his work is acknowledged nationally and internationally. Fully trilingual, he shared his words and prayers in Algonquin, and translated them into English and French. Central to Elder Commanda's teachings are the concepts of equality, balance, respect and responsibility for Mother Earth, for all life forms and for people of all racial and cultural backgrounds, and he worked ceaselessly, alone and entirely without an organization, staff, structure, formal or financial support to animate the Circle of All Nations.

A most senior representative of the Algonquins of the Ottawa River Watershed, he was the great, great grandson of the legendry Pakinawatik, the Algonquin chief who, in the mid eighteen hundreds, led his people from their lands at Oka on the Lake of Two Mountains to traditional hunting and trapping grounds at the confluence of the Desert and Gatineau rivers. William Commanda was the carrier of three sacred Wampum Belts of historic and spiritual importance, originally held by Pakinawatik: the ancient Seven Fires Prophecy Belt about choice; the 1700s Welcoming Belt about sharing the grand natural resources and values of the original peoples with the newcomers; and the Jay Treaty Border Crossing Belt which recognized Turtle Island as a coherent entity. His ancestors inscribed their legends, prophecies and agreements in these carefully crafted items over many centuries. He is viewed by many as the carrier of the Seven Fires Prophecy Belt at the time of the unfolding of its final message; and the messages of all these ancient artifacts are as deeply relevant today, as they were in the past.

He was acclaimed chief of the Kitigan Zibi Anishinabeg for over nineteen years, though he himself never participated in the elections. He also worked as a guide, trapper and woodsman for much of his life. He was a birch bark canoe maker and craftsman of international renown, and there is a special display dedicated to his work at the Canadian Canoe Museum of Peterborough. He built a canoe for Queen Margrethe of Denmark, and he helped Pierre Trudeau repair his famous birch bark canoe. At the age of 90, he shared his canoe making skills and philosophy in Valerie Pouyanne's documentary, Good Enough for Two.

He promoted environmental stewardship and respect for Mother Earth passionately for many decades. He conducted pipe ceremonies for the Pre-Rio Earth Summit Conference hosted by President Mitterand of France in 1991, and his prayers lie behind Agenda 21. He participated in the United Nations first Indigenous Cry of the Earth conference in 1993. He served as spiritual guide to the 1995 seven and a half month Sunbow Five $W$ alk from the Atlantic Coast to the Pacific, to raise awareness of the growing environmental crisis; received the Bill Mason River Conservation Award in 2004; hosted workshops on water stewardship in 2004 and 2006, and 2009; was honorary chair of the Ottawa Heritage River Designation Committee; and offered interventions on current environmental issues such as the identification of the American Eel as a Species at Risk, test drilling for uranium, the building of a mega 
dump on Danford Lake and the Navigable Waters Act.

He was the recipient of numerous awards and acknowledgements of his works and talent: the Wolf Project and Harmony Awards for his efforts to foster racial harmony and peace building through the creation of a Circle of All Nations (one very well received example of this commitment being the annual international gathering he hosted at his home during the first weekend of August - the 2001 Gathering is presented in the Circle of All Nations documentary); a Justice Award from the University of Ottawa and a Peace Award from Friends for Peace. He promoted restorative justice and forgiveness, and his outreach to prisoners is captured in Lucie Ouimet's National Film Board Documentary, Ojigkwanong: Encounter with an Algonquin Seer.

Recently, his efforts were acknowledged in Ottawa with two special recognitions: in 2005, with an Honorary Doctorate Degree from the University of Ottawa, shortly after his book, Learning from a Kindergarten Dropout (Thumbadoo, 2005), was published; and in 2006, with the Key to the City of Ottawa, a singular honour for an Indigenous person from a reserve in Quebec. This was presented on Victoria Island, where the tireless then ninety five year old animated his vision for a National Indigenous Centre, for the restoration and development of the Sacred Chaudière Site as a special national historic centre, and as a think tank for environmental stewardship and peace building of national and global relevance. Two other books, Learning from a Kindergarten Dropout Book Two (Thumbadoo 2008), and Passionate Waters- Butterfly Kisses (Thumbadoo 2008) include further reflections on his work and ideology.

In December 2008, he was appointed Officer of the Order of Canada, for his leadership as an elder who promoted intercultural understanding and raised awareness of the traditions and legacies of Indigenous Peoples. Elder Commanda was deeply honoured to witness this recognition of the relevance of Indigenous wisdom to this country.

In November 2009, the National Aboriginal Achievement Awards Foundation announced his selection as 2010 Lifetime Achievement Award recipient. In January, 2010 Willis College announced the Dr. William Commanda Scholarship! In November 2011, he was presented with a doctorate honorifique by l'université du Québec-Outaouais posthumously.

The following 3.52 minute video clip of William Commanda, created by his Circle of All Nations colleague SunBow, Iron Wood Log Project, entitled Tribute to the legacy of a legendry peacekeeper offers a mini resume of his work https://youtu.be/Psax2u_rDO4

\subsection{Guardian of Sacred Wampum Heritage}

William Commanda became carrier of three sacred wampum belts of spiritual, historical and political significance in 1970. The following excerpt from our book, Learning 
from a Kindergarten Dropout, provides a contextual description:

From the earliest of times, William's ancestors along the eastern seaboard of North America created belts woven with beads made of the purple and white shells of the marine sea creatures. The word wampum emerges from the Algonquin word wampumpeage which described white shells and or strings of shells; its roots link also with the words waban and wabanpaog, referring to the dawn or the first light of the east, and the eastern people. The belts that William carries are predominantly purple; the beads were made from the shell of the quahog. It is not clear how they fashioned the thousands of tiny beads before the age of industrialization, but it is well known that they created many beautiful belts that served as devices to record their stories, legends, prophecies and agreements. These served both as a living record of a commitment and also a means to recall the messages (Thumbadoo 2005 49).

The wampum belts were the mnemonic devices or memory awakeners, ideographic rather than phonetic. They complemented language and oral delivery in communications.

With reference to bark scrolls, the 1800s anthropologist Schoolcraft, writer of Historical and Statistical Information Respecting the History, Condition, and Prospects of the Indian Tribes of the United States (1851-1857), (who learned Algonkian, married the daughter of an Ojibwa chief, and was admitted as member of the Midewiwin medicine fraternity), offers an explanation of pictography, that can be applied also to an understanding of wampum devices, as:

"the mode of communicating all classes of ideas commonly entertained by them. So considered, it reveals a new and unsuspected mode of obtaining light on their opinions of a deity, of the structure or cosmogony of the globe, of astronomy, of the various classes of natural objects, their ideas of immortality and a future state, and the prevalent notions of the union of spiritual and material matter. So wide and varied, indeed, is the range opened by pictography, that we may consider the Indian system of figure-writing as the thread which ties up the scroll of the Red Mans' views of life and death; that it reveals the true theory of his hopes and fears, and denotes the relation he bears, in the secret chambers of his own thoughts, to his Maker... These figures represent ideas - whole ideas, and their juxtaposition or relation on a scroll of bark, a tree, or a rock discloses a continuity of ideas. This is the basis of the system (Schoolcraft in Vastokas 1973 43).

After the arrival of the newcomers, the Europeans manufactured beads for trade, and the belts became used as items of trade and commerce by other tribes, most notably the Iroquois. Most of the old belts, the original ones and the newer creations, have been 
expropriated to museums and churches, and their absence is a sad, destructive and shameful statement in a country that celebrates the heritage of the many newcomer cultures on the land.

The three (Algonquin) belts, together with another one that disappeared many decades ago, and several strings, were held by WC's great, great grandfather, Pakinawatik, their oldest known guardian, and they carry testimony of significant prophecies, agreements and understandings which guided the Anicinabe peoples for centuries. They inspired WC's work for over forty years. In 1987, he began sharing the messages of the wampum belts publicly during the constitutional debates in Canada, and he continued to do so till his death. He stated repeatedly that the three belts he carried were living entities because they were not housed in museums, and during the latter period of his life, he shared their messages with increasing urgency. This is consistent with the prophecies circulating in the eighties that spoke of the return of the voice of Indigenous Peoples.

WC was carrier of the Algonquin Seven Fires Prophecy Wampum Belt, an epic oral story that spans space, time and metaphoric domains. The general authenticity of oral stories can resonate across time and space; when WC met Zulu Shaman and writer, Credo Mutwa in South Africa, he stated that the same prophecy existed with his peoples too. However, there are different pathways into the oral story thread, and WC came to his understanding and knowledge of this pivotal prophecy via his parentage from both his father's and mother's direct family lines, and from his animative relationship with them, this being aligned with nature-spirit energy that he affirmed.

The core ideographic messages of the three Wampum Belts, excerpted from Learning from a Kindergarten Dropout are shared here:

The Seven Fires Prophecy Belt 
Elder Commanda is believed to be the keeper of this ancient sacred belt (relating events) dating back to the late 1400s at the time of the unfolding of its final message - the message of CHOICE - about our relationships with each other and with all creations of Mother Earth. Will we be guided by values of sharing, balance and harmonious co-existence?

\section{The 1700 s Welcoming Belt}

In this three figure wampum belt about equitable SHARING, William Commanda's ancestors inscribe their understanding about sharing the resources of their native land and their values and ideology with the newcomers, the French and the English, in the spirit of a confederacy. The inherent value of sharing remains the elusive quest of our times.

\section{The Jay Treaty Border Crossing Belt}

This belt underscores the fundamental spiritual message of Indigenous peoples about Borderlessness: the Elder's people, the Mamiwinini, the nomads, belong to North America, and as such they retain a sacred connection and responsibility to the land they are born to. As Elder Commanda puts it, "My territory is as the river flows, as the bird flies, and as the wind blows."

[This belt was created in the 1790s during the time of the negotiation of the Jay Treaty and affirms the right of Indigenous Peoples to cross the border 'without molestation' (William Commanda's words); he stated that the inclusion of artificial white Hudson Bay beads asserted the conviction of his ancestors that borders were an artificial creation and would eventually disappear; WC worked to animate the Indigenous values represented in the backdrop of the purple beads across both Canada and the United States] (Thumbadoo 2005 50).

He brought the belts out publicly for the first time in 1987, thirty years ago, asserting

Indigenous law, governance and Law of Nature mandates, to launch the fourth of the

Aboriginal Constitutional Conferences hosted by Prime Minister Brian Mulroney. This had been committed to in 1982, when Canada and Prime Minister Trudeau brought the Constitution home, enacted the Constitution Act and enshrined the Charter of Rights. Thereafter, WC delivered their messages at the first United Nations Conference of the World's Indigenous Peoples (1993), called, pointedly, Cry of the Earth; at the Circle of All Nations International Millennium Peace Gathering; at his annual international Circle of All Nations gatherings; at federal government meetings on critical policy and program initiatives 
or events; and at hundreds other meetings with individuals and groups. Thus, he singlehandedly ignited a relationship with this tangible and intangible heritage of his ancestors for a vast number of peoples and institutions. A critical agentive discursive element in his outreach, its impact is evident at multiple levels today.

The following video of the 1999 Prayer Vigil in Washington DC provides a brief overview of his wampum teachings (Wittenberg Centre, Woodstock, New York): https://www.youtube.com/watch?v=h0Vs8OmxLcA

The following list itemizes some of the acknowledgments of his knowledge and wisdom:

- Carrier of Sacred W ampum Belts

- Nineteen years of service as chief by acclamation

- Supreme Chief North American Indigenous Nations Government

- Receipt of two Honorary Doctorate Degrees

- Appointment as Officer of the Order of Canada

- Master Canoe Builder Award

- Wolf Project and Harmony Awards for promotion of racial harmony

- Friends of Peace and Universal Peace Federation peace awards

- Martin Luther King Dreamkeeper Award

- Bill Mason River Conservation Award

- Bernard Asiniwi Indigenous Leadership Award

- $8^{\text {th }}$ Fires/Turtle Lodge Indigenous Elder Award

- National Aboriginal Achievement Lifetime Award

- Key to the City of Ottawa

- Remise du prix Pacifiste de l'anne e au chef Algonquin W. Commanda, Montreal

- Willis College Dr. William Commanda Scholarship

- University of Ottawa Medical School Dr. William Commanda Scholarship, and

- City of Ottawa's Reconciliation Day acknowledgement (post humous)

These and other formal and informal acknowledgments of his leadership and reconciliation work attest collectively to his engagement and achievements on a range of contemporary issues.

He is considered by many to have impacted significant shifts in thinking on environment and relationship issues at local, national (Canada), global and political levels. 
During the last two decades of his life he was a prominent participant in government and UNESCO meetings and international conferences. Significant contributions in this regard include his impact on

- North American Indian Nations Government from the 1940s

- 1987 Constitutional Debates

- 1990 United Nations Pre-Earth Summit Conference in France

- 1993 United Nations Cry of the Earth Conference of Indigenous Peoples

- Annual Peace Vigils in Washington DC

- Funai Earth Conference, Japan

- 2000 UN Peace Conference

- 2001 United Nations Conference for the Elimination of Racism and Xenophobia, South Africa

- 2001 Systemic Constellation Conference, Germany

- Shivananda Yoga Centre Peace Conference, Bahamas

- 2004 Launch and Federal Roundtables on Species at Risk. Act

He led important local campaigns on environmental issues

- served as spiritual guide to the 1995/6 Sunbow Five Walk across America to raise awareness of environmental issues, historical injustices, racial harmony and peace building

- challenged contamination of the Ottawa River (Waterlife Workshop 2006)

- advocated for registration of the American Eel on the Endangered Species list (2007)

- challenged expansion of hydro development at the Sacred Chaudiere Falls (2007)

- challenged uranium drilling impacting the Ottawa River Watershed (2008)

- challenged the creation of a megadump at Danford Lake, Quebec (2008)

- organized two workshops on Sustainable Relationships on Victoria Island (2009)

- challenged the Navigable Waters Act amendment

The following brief listing of WC's engagement in environmental issues over the years attests to his passionate relationship with Mother Earth, and his commitment to asserting his views and inspiring or instigating shift on national and international platforms with multiple partners, both Indigenous and non-Indigenous.

Chronology of Key Activities Organized to Advance Stewardship of Mother Earth:

1945 - Precursor - Indigenous Petition on Environment to the UN

1967- First Indigenous Gathering - at Eganville, Ontario

1969 - Gathering of Nations in Maniwaki 
1987 - Presentation of $W$ ampum Belts at Constitutional Talks

1991 - Pipe Ceremony at Pre Rio Preparations in France

1992 - Message to Earth Summit in Rio - Agenda 21

1993 - Cry of the Earth Conference at UN

1995 - Spiritual Guide of the Sunbow Five Walk for Mother Earth

1996- Release of the Royal Commission on Aboriginal Peoples Report at Victoria

Island (where he raised concerns about the pollution of the Ottawa River)

2004 - Honorary Elder - Boreal Rendezvous

2004 - Heritage Rivers Bill Mason Conservation Award

2006 - Honorary Chair - Ottawa Heritage River Designation

2006 - WaterLife Workshop, Ottawa, Ontario

2007 - The Awakening Gathering, Perth, Ontario

- Annual Circle of All Nations Gatherings: environmental stewardship;

2002 and 2004 Gatherings focussed on Water, Kitigan Zibi, Quebec

- Annual Gathering of Nations Pipe Ceremonies at Victoria Island, Ottawa

- Interventions on the Proposed Megadump at Danford Lake,

- Sacred Chaudiere Site Legacy Vision: Indigenous Eco Peace Think Tank

- American Eel as a Species at Risk

- Challenge of Test Drilling for Uranium

What was innovative and ground-breaking in WC's approach was his engagement of all, (Algonquins, First Nations, Inuit, Metis, Status and Non-Status, On- and Off-Reserve and non-Indigenous peoples), in his Circle of All Nations work. His efforts may be viewed as a determined counter-discourse to the othering Edward Said had exposed in his influential book, Orientalism. Said had argued that the Oriental was a deliberate creation of the West in its scholarly and imaginative writings, to maneuver imperial designs (Said 1978). William Commanda initiated his bridge-building strategy formally with his first gathering hosted in Eganville, Ontario in 1967, fifty years ago, on the occasion of Canada's hundredth birthday. In 1969, and thereafter, demonstrating conscious agency, he hosted gatherings at his home in Kitigan Zibi Anishinabeg (then River Desert Reserve); after 1995, these attracted thousands of people from across the globe each year. He hosted four major and influential conferences in the Ottawa area (1998 Wolf Project Workshop Lighting Candles for a Culture of Peace, 2000 Circle of All Nations International Millennium Peace Gathering, 2006 Waterlife Workshop, and 2007 Awakening Conference). He held several pipe ceremonies, paddle for 
peace events and sustainable relationship workshops each year on Victoria Island, from 1997 till his death. He also supported all events as requested by others, irrespective of how humble or highly profiled. In this process, he challenged the underlying structures of power, inclusion and exclusion in colonial and imperial social networks, including by shifting power at a 1990s meeting of the British Commonwealth Society in the capital city with his emphatically Indigenous presence. His emergent presence is tangible today in the cyber world and social media, six years after his death, in diverse discourse on environment and peace.

He advanced new ideas and thinking on Indigenous environmental stewardship and relationship, still deemed relevant by many today. WC located his discourse in Law of Nature semantics, around a conceptual Circle of All Nations version of a medicine wheel, presented graphically to the public at large since 2000. WC's work is an important representation of the Indigenous thinking of his ancestors, and provides a comprehensive example of such thinking. However, it is not representative of all Indigenous thinking on the issues examined. Comprehensive records of his work are held in the Circle of All Nations and Asinabka website archives (www.circleofallnations.ca; www.asinabka.com), and have been on-line since 2000. (Note: all material in the websites, with the exception of those documents credited to specific individuals, have been authored by me, since 1997, under the names Thumbadoo and Trebilcock, in collaboration with William Commanda until his death on 3 August, 2011, and alone since then). WC and his Circle of All Nations work are also a vibrant presence on Facebook social media, (where I host the following Facebook pages: William Commanda, William Commanda Legacy - Asinabka, Circle of All Nations, and Romola's Nature CAN TEACH), and in the web at large, as input by others.

The extensiveness of this outreach and his voice through his Circle of All Nations 
work sets the context for the central question about his discourse and its relevance to the academy. What this thesis seeks to determine is whether there is potential and genuine relevance for the inclusion of William Commanda's thinking, knowledge and knowledge generation approaches in academic literature on the environment and relational issues.

\subsection{Situating Myself}

I now explain briefly why I am in a unique position to research and document William Commanda's ideas. I met William Commanda in 1997, in the context of my work as a federal public servant responsible for national federal/provincial/community initiatives in Aboriginal justice, policing, corrections and restorative justice. This was during the time of the Oka Crisis, the Donald Marshall Junior and Manitoba Aboriginal Justice Inquiries and the Task Force on Federally Sentenced Women, a time of national awakening to the systemic and overt racism and injustice impacting the lives of First Peoples. It was also a time of global awakening to the looming environmental crisis, with the United Nations introducing the idea of sustainable development to the world. When I understood the scope, context and urgency of William Commanda's work, I took early retirement to support his unfunded Circle of All Nations initiative pro bono. I interacted closely with him on a personal level from April 1997 until his death in August 2011. This engagement with all aspects of his life and work, and his repeated iterations of his priorities and memories directly to me over a fourteen year period presented me a unique opportunity to understand his thinking, as well as a responsibility to record and share it. The past five years have been committed to the scrutiny and research of his ideas and work.

In 2005, I wrote a book entitled, Learning from a Kindergarten Dropout (Thumbadoo 2005), discussing his key teachings. William Commanda was an avid student and a firm believer in lifelong learning; he was also a determined teacher. The idea of recording his 
thinking and knowledge in a more formal way began to register in our consciousness a decade ago. In 2007, we participated in the launch of the Lake Huron Treaty Digital Atlas project at the Geomatics and Cartographic Research Centre (GCRC) at Carleton University. Both of us noted that the digital platform would be an exciting way to present his story. William Commanda was already well known as the dot.com elder by then. He was 94 years old at the time. We engaged in brief discussions with Dr. D. R. F. Taylor, Director of the Centre, and the internationally acknowledged father of cybercartography. In 2009, we engaged in exploration of a potential digital atlas research project, and then followed this with formal application to doctoral studies by both of us. Unfortunately, this did not materialize at that time. After William Commanda's death in 2011, I, by chance, attended another event at the GCRC, and the plan to locate his knowledge more formally within the academy arose again. Quite literally at the eleventh hour in September 2012, I applied for admission to the Department of Geography, to pursue this thesis research, which William Commanda had himself hoped to undertake earlier.

\subsection{Thesis Presentation}

The thesis is presented in the following way:

Chapter 1 provides a brief introduction of William Commanda's background, including his academic interests, my situatedness in the research, and this overview of the Thesis Presentation;

Chapter 2 discusses the Theoretical Framework and Structural Parameters of the thesis;

Chapter 3 discusses the Methodological Tool Kit;

Chapter 4 examines the discourse from the first logic of inquiry: performance mapping geo-narrative;

Chapter 5 examines the discourse from the second logic of inquiry: historicaldialectical narrative; 
Chapter 6 examines the discourse from the third logic of inquiry: phenomenological reflective auto-ethnography;

Chapter 7 examines the discourse from the fourth logic of inquiry: cybercartographic narrative; and,

Chapter 8 presents conclusions, and discusses emergent questions and projections for future research.

Chapters Two and Three on theory and methodology provide general overviews of the four logics of inquiry selected to undertake the research. Each of the theoretical entry points and the associated methodological tools are examined in much greater detail in Chapters Two, Three, Four, Five and Six, to accommodate the challenges of writing abstractively while embedded in a emergent oral narrative. The extensive discussion provides an optimal entry into the Indigenous discourse of William Commanda. It is designed to substantiate the subsequent detailed examination of the pedagogical framework of his cyclical narrative teaching style and assess its bridge-building and integrative potential. In this way, I strive to integrate William Commanda's theoretical position which entails looking at the world wholistically, grounded in experience with the natural world, revitalized through ceremonial reflectivity and incorporated into the spiritual dimension of his methodology.

Each of Chapters Three, Four, Five and Six include introductory remarks, discussion of the selected theoretical lens and the methodological tools, a conceptual map, discussion, analysis and observations on four key themes, conclusions, summary of findings and a related Commanda exemplar.

\section{Notes}

1. I refer to William Commanda as William Commanda or WC in the thesis.

2. I use the words Algonquin, Algonkin, Algonkian and Algonquian, and different names for Indigenous Peoples, to be consistent with the literature, issues and time periods under examination in different parts of the thesis.

3. William Commanda used the term Mother Earth in his discussions. I examine the word environment on page 45, noting that while it does not emcompass the thinking of WC, it is used in the thesis as a bridge to approximate, negotiate and explore a field of common interest. 


\section{Chapter 2 \\ Theory}

\subsection{Theoretical Framework}

\subsubsection{Locating the Research in Geography, Cartography and Cybercartography with Linkage to Environment}

This research is being undertaken in the Department of Geography and Environmental Studies, with direct links to the Geomatics and Cartographic Research Centre and to cybercartography. It seeks to understand William Commanda's relationship with land, mapping, environment and certain contemporary socio-ecological issues. A discussion of interrelated geography, cartography, cybercartography and environment themes inform this research.

Respect for and relationship with the environment and people is central to the work and Anicinabe teachings of William Commanda and other Indigenous Peoples. This approach is grounded in an empirical, experiential, collaborative and observational scrutiny of the Laws of Nature and diversity, with knowledge generation emanating from this source.

A deeper understanding of the earth-connected foundational approach of people like William Commanda can serve to strengthen linkages and integration of Indigenous approaches in mainstream academic discourse. It can also contribute to "the reflexive analysis and discussion of values and interests, which is the prerequisite for enlightened political, economic and cultural development in any society" (Flyjvberg 2011 5), and can impact environmental studies productively.

\subsubsection{The Research Question}

The central research question that this thesis examines is William Commanda's thoughts on Mother Earth, environmental and relational issues, how they have informed contemporary debates on Indigenous and 
non-Indigenous relations and thinking, and whether they can contribute to a greater contemporary integration of his Anicinabe (Indigenous) approach to knowledge generation in academic discourse.

William Commanda created a Circle of All Nations, an informal global eco-peace community, over the course of the past fifty years. It is premised on the Indigenous integrative principles of a cosmic and ecologically bio-diverse, hybrid, emergent worldview, as animated by him. His Circle of All Nations is a zone of inter-connection, permeability, reciprocity, exchange, transference, transformation and co-creation, wrought in a field of contraries and friction, repeatedly negotiated and re-birthed via the assertion of relationality: Ginawaydaganuc. This was his word to describe the concept of a multi-dimensional relationality inclusive of a biotic relationship with a living and constantly evolving Mother Earth. I argue that he created the Circle of All Nations deliberately as a bridge to encourage new approaches to environment and relationships in a world in severe crisis, in view of the global escalation of environmental degradation, racism and war. He observed the interrelationship between these destructive forces and responded to this in a variety of active ways from the mid-nineteen forties, from localized interventions to outreach at the global (United Nations) level.

The focus of the study is both theoretical (whether and how William Commanda's discourse intersects with approaches to environment and historical and contemporary issues of imperialism, colonialism, land appropriation and degradation, racism and justice); and action oriented (regarding the continued relevance, animation and emergence of the discourse through agency and praxis to advance environmental stewardship and peacebuilding), in its examination of his animation of Ginawaydaganuc and relationality at multiple levels to influence change. Linda Tuhiwai Smith writes that "Systemic change requires capability, leadership, support, time, courage, reflexivity, determination and compassion. It is 
hard work and the outcome often seems a distant vision. Paulo Freire referred to this as praxis: theory, action and reflection; Graham Smith has called it indigenous transforming praxis" (Tuhiwai Smith 2012 xiii). WC's strategic approach was guided by both his lived experiences and his understanding of his responsibilities as carrier of the ancient sacred wampum heritage of his ancestors. Consistent with WC's own storytelling style, derived from Indigenous oral tradition, and his use of this approach to teach and educate peoples of diverse backgrounds, including many academics, I undertake a theoretical analysis of a singular geonarrative storytelling case study deeply embedded in time and space.

Over the past two decades, Circle of All Nations has used its version of a conceptual medicine wheel to advance the cross-cultural bridge-building teachings of WC, in the development of its own projects and activities, in its written materials and publications, in its website design, its graphic presentations, and in formal and informal academic/educational events, conferences and workshops. In essence, it employs a cyclical planning and activation model that moves from visioning, to animation, to refining, to dissemination of ideas. The thesis employs this Circle of All Nations medicine wheel conceptual framework aligned with four logics of inquiry to undertake the research:

- Performance mapping: to explore the ideological framework, Anicinabe underpinnings and Indigenous epistemology; in essence, this is the vision matrix, and the research explores the backdrop that informed William Commanda's thinking;

- Historical/dialectical: to ground the analysis of his agency in poststructuralism and Indigeneity and examine its inseparability from colonial history, injustice and inequality, as well as the challenge to provoke change; this is the zone of activation, and action, and the research examines the William Commanda's work and outreach from this perspective;

- Phenomenological: to substantiate an interpretative approach to analysis, inclusive of auto-ethnography, critical reflexivity, and reciprocity; this involves review and refinement; the research traces the processes of attentive reflexivity that ensure an evolving dynamism in his approach;

- Cybercartographic: to examine multi-dimensional reporting, archival, graphic and contemporary emergent elements; this involves the dissemination of knowledge, information and energy to stimulate new ideas and thinking. 
I use geo-narrative, narrative, reflexivity and cybercartographic methodological tools used by him to probe WC's approach to knowledge generation in contemporary times, and to explore whether and how such research and scholarship might contribute to the creation of new pedagogic approaches and impact academic thinking on challenging, contemporary socio-ecological issues.

\subsubsection{Circle of All Nations Logo as Theoretical Frame}

The Circle of All Nations logo, formally utilized as an epistemological tool since its creation in 1997, serves as illustration of the general thesis framework. The tree was photographed by William Commanda; the turtle was a live turtle photographed by Claude Latour, and the logo was co-created by Romola V. Thumbadoo, William Commanda and Claude Latour.

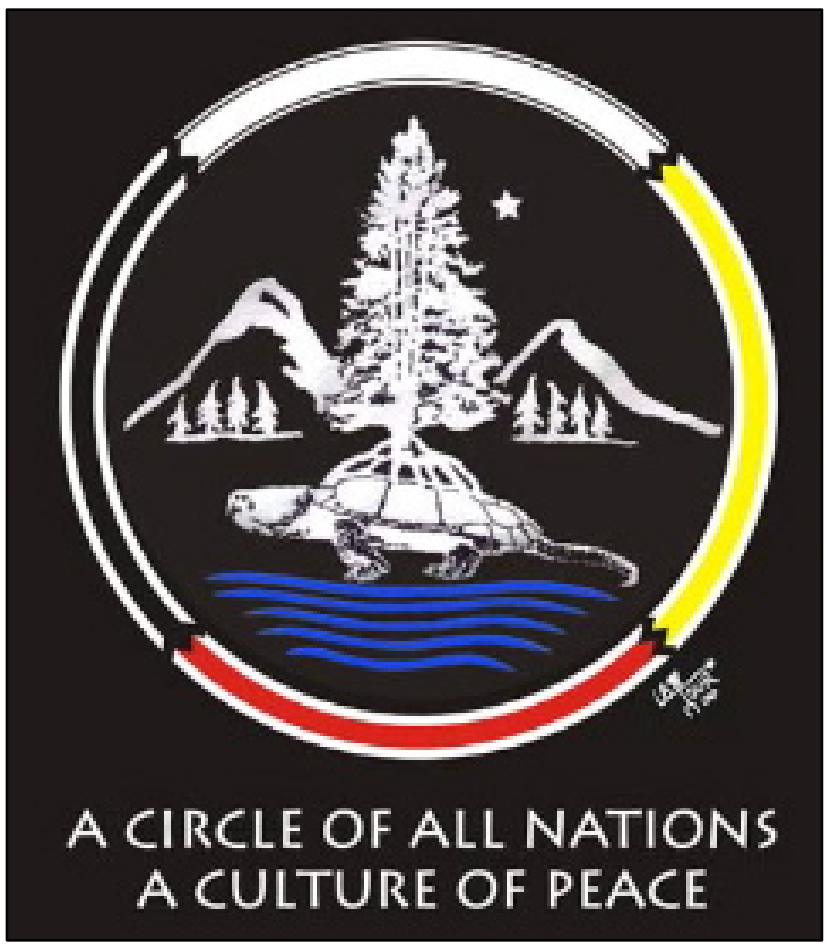

Figure 2. Circle of All Nations Logo. 
The Circle of All Nations Logo, as articulated in 1997, offered the following description.

This special logo incorporates many symbols of special significance to Aboriginal and non-Aboriginal peoples and to William Commanda himself.

The four colours of the Sacred Circle, at the simplest level, represent the four symbolic races of man, the Yellow, Red, Black and White peoples; the four cardinal directions, East, South, West and North; and the four elements, Fire, Earth, Water and Air. The symbolism and teachings of the Sacred Medicine Wheel are profound.

The Turtle, here representing Turtle Island, North America, is described similarly in the mythology and legends of Aboriginal peoples of the West, the Hindus of the East and the Zulus of the South, amongst others, as being the animal that sacrificed its life to create Earth in the expanse of Sea and Sky; it endures as a symbol of sacrifice, creativity and fertility. The Western story of the Tortoise and the Hare stresses the importance of perseverance in following dreams and achieving goals.

The Sacred Tree is a Spruce Tree photographed by William Commanda. Four separate trunks emerge from one base of the 75 foot tree. The seven roots of this tree, which reaches into the Sky, grow through the Turtle into Mother Earth and the Sea, connecting all. It represents the Seven Fires Prophecy of the sacred wampum belt William Commanda carried for the people for over forty years.

The other trees remind us of the importance of the trees to Mother Earth and to our lives. As he watched with pain the endless passage of logging trucks through his family's traditional lands, William Commanda said, "It feels like a needle piercing my eye".

The Mountains represent The Lake of Two Mountains, the traditional homeland of William Commanda's ancestors. They also represent the climb up the Sacred Mountain in the search for wisdom.

The Morning Star is the symbol of enlightenment and vision, and because it shone so brightly when he was born, his mother named William Commanda "Ojigkwanong". (Thumbadoo 2005 54)

With specific reference to this thesis, I articulate the significance of the logo as follows:

- The Morning Star bridging between night and day, asserts the energy of emergence, enlightenment and knowledge, of the aspirational, and of volition and willpower;

- The image backdrop denotes a cosmic stellar world inextricably linked with sacred earth, Turtle Island, with semiotic messages of water, turtle and prophecy roots performance mapping the ideological foundation, cartographical geo-narrative, reflexivity and cognitive mapping themes of the thesis;

- The cosmic spruce tree (photographed by William Commanda) is used in the creation of the iconic Algonquin birch bark canoe. It also reflects the relational/inter-connectedness/adhesive theme of Ginawaydaganuc, around which the 
entire thesis spins;

- The territorial referential of two mountains underpins the historical dialectical spatial narrative; the cosmic tree links elemental creation stories with historical references (Lake of Two Mountains at confluence of Ottawa and St Lawrence Rivers); and the special biodiversity/environmental importance of the area and work is anchored by trees;

- The four separate trunks are aligned with the four branches in the ways of knowing examined in the thesis: empirical, animative, reflexive and dialectic. Analysis of research materials in the context of the centrality of the Ginawaydaganuc/relational theme is conducted in part via the digital atlas methodological trunk tool; and the tree branches epitomize the cybercartographic and social media refractions and voices.

- The circle outline demarcates the constructed circular storytelling epistemological approach which, with distinct association with colour (from [en]lightenment of the yellow of the East, to the passionate animation of the red of the South, to the reflexive zone of the black of the West, to the wisdom articulation in the white of the North) projects movement and flow, the underlying regenerative theme of the thesis. A further examination of the circular Medicine Wheel flow reveals the movement from visioning and planning, to animation and action, to reflection and refinement, to articulation and dissemination as wisdom.

- As intimated and articulated, the Circle of All Nations projects the ultimate vision of balance and harmony, emergence and evolution to be achieved in the motion of the striving for a Culture of Peace.

\subsubsection{Thematic Context}

The thesis explores the following themes:

1. The interplay of William Commanda Law of Nature/way of life indoctrination. This was informed by his land-based language, culture and heritage, and articulated ideologically and epistemologically through rock art, wampum belt agency, and ceremonial and ritual practices. It was manifested in his absolute adherence to a central principle of interrelatedness and oneness seen within an infinitude of diversity, conceptualized around Ginawaydaganuc (that all, including humans, are related in the geo-cosmic world, and therefore one);

2. His contemporary locatedness in a colonized Europeanized world rendered paradoxically both globally connected and multiply divided. From the time of his birth on the eve of the First World War, his world was dominated by ideologies of duality manifested in a zeitgeist very different from his foundational embeddedness in inter-connection. Yet, ironically, over the course of his own lifetime, it has also become a globalized world, one that is now grappling desperately to come to terms with diversity;

3. His Indigenous understanding and animation of syntropy and negentropy, consistent with the cosmic principles of space/time, movement, causality, energy, life, creation, entropy, retrocausality, emergence and evolution. He consciously, deliberately, and determinedly animated of his own theoretical platform and research project in the creation of his Circle of All Nations global eco-community as a bridging device negotiating between differing worldviews. Here he worked to reconstitute and 
reconstruct relationships consistent with the laws and intelligence of nature, and with principles of creativity, resuscitation (restoring consciousness) and reciprocity (exchange); and

4. The positioning of his Law of Nature approach to knowledge generation on environment and related matters. He engaged in knowledge creation on multiple platforms, local, regional, continental and global, and utilized traditional and evolving technologies to generate engagement in and further animation of his project, which he considered of critical importance to Mother Earth and all creation.

\subsubsection{The Logics of Inquiry}

The examination of William Commanda's discourse requires scrutiny from a variety of interpretative and methodological lenses to ensure that the research addresses both the objectives of adherence to its Indigenous integrity, as well as the exacting requirements for credibility and relevance in the western academic regime, in determining a possible place of integration. Michael Watts, in The Holy Grail: In Pursuit of the Dissertation Proposal, addresses the importance and difficulties of formulating a coherent, conceptually integrated and empirically grounded research question that commits the scholar to mastering literatures, identifying with a theory, and working with data (Watts 2001 7). The crux of the challenge at hand lies in bridging Western academic and theoretically grounded approaches with empirically and esoterically grounded Indigenous knowledge, and identifying any zones of interface. Watts points to the critical importance of selecting the appropriate entry point or logic of inquiry in order to link theory and evidence. He notes further that "in practice a research project may deploy two or more ... logics of inquiry - great works typically do and a research program may indeed be involved in using specific data in a variety of ways (if possible) to make it appropriate for different types of analysis” (Watts 2001 10).

I explored a range of academic material that has impacted geographic and cartographic studies since the launch of the History of Cartography encyclopedia series in 1987. These dispatched me on a trail over conceptual space and time from the ancient expressions of Indigenous cartography to the emergent zone of the cyber atlas, to track the signposts 
that would enable me to identify appropriate tools to address my research question. I liken the experience to a canoe journey over uncharted territory. I have had to ignore or abandon many tributaries in this journey. The ones that the current guided brought me to this crossroad and configuration of theoretical tools.

I offer some further discussion of the methods selected for this research project, demonstrative also of the cyclical approach. I employ four proposed logics of inquiry and selected associated methodologies as follows:

- performance mapping, and geo-narrative

- historical/dialectical approach, and narrative

- phenomenology, and auto-ethnography and critical reflexivity

- cybercartography, and cybercartographic digital atlas and social media

I approach the logics of inquiry in a cyclical fashion, moving from one lens to the next. The first lens of performance mapping explores the grounding Law of Nature worldview, and examines the agency of earth, rock and river, oral, language, text, semantics, symbol and semiotics, as well as wampum, rock art and iconography. The second involves a historical/dialectical approach in the examination of the Indigenous and textual narrative. Here I study WC's life and narrative embedded in the web of inseparable, conjunctures, contextualities, contingencies and contradictions from historical times of contact into the present. The third lens positions our respective interpretive focalization and the perspectives through which the narrative is presented in a phenomenological logic of inquiry. Here I rely on auto-ethnography and critical reflexivity, field and participant observation, nominalization, positionality and cultural interpretation. Finally, the fourth lens accesses cybercartography, reflecting the interplay of space and time, groundedness in the land, travel and movement, physical and sensory data, and individual and multiple perspectives, accessed in textual, photo/video/audio, and social media material both produced and emergent in a processoriented contemporary discourse. 
The associated methods follow the same cyclical flow. Firstly, the geo-narrative addresses the 'Law of Nature' framework, emergent from land, language and associated ways of knowing, some re-constituted in wampum agentive law, logistics and animation. Secondly, the narrative explores specific components of the WC's discourse and his deconstructive and decolonizing work from the historical/dialectical lens. His long life, library and archives of a century of tremendous global change provide a rich record for research and analysis. Thirdly, the parameters of auto-ethnography and critical reflexivity permit the unique interpretative analysis of the discourse. I study WC's own stories, messages and cyclical repetitions, iterations and reflexivity in video, audio and written material, including my own handwritten and typed notes of conversations with him. Then I analyze critically my intensive engagement in Indigenous issues for over thirty years, and in WC's work for over twenty years, my academic background in literature and study of language, my unique insider/outsider status in his work, and my articulated reflexivity in photos, creative writing and video materials. Finally, fourthly, the creation of a cybercartographic atlas draws on digital mapping and social media to examine the multiple voices/player/actants, engagement and emergence evident over space and time in the William Commanda/Circle of All Nations discourse. This contemporary expression of performance mapping propels the cyclical research project to the emergent spiral in the conceptual Circle of All Nations medicine-wheelin-motion. For the purposes of the thesis research, only key elements of Commanda discourse pertaining to his relationship to sacred earth will be examined here.

\subsubsection{Discourse Analysis Approach}

French philosopher Michel Foucault created a tremendous surge of new academic analysis in the social sciences around the term discourse, contextualized in poststructural thinking of the seventies. As Cresswell (2009 211) points out, at one level, discourse is the 
term used to refer to spoken or written text or communication about a particular topic. Barrett's discussion of textuality in The Politics of Truth deepens this consideration. She points to contemporary acceptance of language as the producer of knowledge, with the Anglo tradition further establishing written language, in its silent manifestation, as the vehicle for the clearest, most truthful, expression of meaning. Written tradition undercuts the oral, as well as other performative means of creating and communicating meaning. Further, both the written and oral text of literary cultures is contingent and vulnerable to manipulation, hence alertness to contextuality is critical (Barrett 1991 124-125).

Foucault, however, explored something larger in his construct of discourse, in the process challenging many previously established theoretical frames. These ideas dislodge theories about ideology, social formation and class, etc., and set up discourse analysis as a more reliable means of studying social phenomena. Hence the tremendous interest in this approach. It is interesting to note that the word itself, in its Latin roots, discursus, running to and fro (Collins Westminister Dictionary, 1960), appears to anticipate Foucault's presentation of discourse as dynamic.

In essence, Foucault pointed to an analysis of human/social situations in the interplay of power, knowledge, knowledge creation and presentation of truth. This is manifest in ideas, interpretations, relationships and technologies that funnel a momentum towards an emergent reality. This co-creates a reality of its own, within which the created knowledge becomes dominant and common sense. This is Foucauldian discourse (Waite in Hay 2010; Cresswell 2009). As Barrett elaborates, Foucault presented a discursive field characterized by systems of dispersions and he examined the systematic presence of these dispersions within the discursive structures (Barrett 1991). The more 'true' the contributing factors are interpreted as or accepted as being, so they inform and stabilize the creation of 
the strengths of the discourse. Thus, in his work, Foucault took the conventional linguistic definition of discourse beyond the confines of the writing of speech to an exploration of the creation, production, authentication and circulation of knowledge, and presentation of truth.

In Making Social Science Matter, Flyvbjerg discusses Foucauldian ideas of power, knowledge and discourse, and he points out that one ought not view the universe as divided into accepted and excluded discourse, dominant and dominated discourses, but rather, one must note that all societies have their regimes of truth. Thus, one must also note the multiplicity of discursive elements put into operation in various strategies. It is the distribution of this multiplicity of discursive elements that must be deconstructed and reconstructed in the study of power, noting what is articulated and what is invisible and what relations are inherent in the field (Flyvbjerg 2011).

I argue that in his creation of the Circle of All Nations as bridge between Indigenous thinking and diverse other societies with their regimes of truth, WC animated a new discourse and his regime of truth was grounded in the Laws of Nature and relationality with Mother Earth. Consistent with these ideas, the approach used for the study of WC's work is via a discourse analysis, through which the central argument of this thesis is examined and around which the research is structured. Further, peripherally, it touches on Foucauldian genealogy in its examination of the emergent earth-based origins of WC's discourse. WC's discourse is deconstructed. Then its potential relevance to the academy is examined in its reconstruction as a conceptual Medicine Wheel. This research is not a detailed examination of Foucault's analysis of discourse.

\subsubsection{Case Study}

It is interesting to note that in Germanic languages, science means literally, to create knowledge. In Making Social Science Matter, Flyvbjerg notes that the formal generalization 
associated with western scientific research is only one of many ways people create and accumulate knowledge (Flyvbjerg 2011). There are also the approaches that inform Indigenous ways of knowing. They include, for example, the experiential and empirical, the individual and collective process of observation, knowledge accumulation, collaboration and confirmation, and the ceremonial and relational, all consistent with the Laws of Nature. Since such knowledge was wrestled by WC's ancestors in the harsh and changing conditions of glacial retreat, and critical to life and survival, it was meticulous, Indigenous Law of Nature science. However, the thinking, ontologies and epistemologies of Indigenous peoples on environment and related issues have not been effectively integrated into the academic discourse on these topics. Despite rhetoric to the contrary, Indigenous thinking exists largely outside the current dominant discourse for a number of reasons, including because of the complex semantics and interplay of language, symbols, dream and logic. Escalating environmental degradation preoccupied WC (and countless other Indigenous peoples) for close to a century. These times of global crisis and extreme weather call urgently for heightened respect, understanding and integration of Indigenous science, knowledge and ways of understanding environment and relationship.

Drawing on the perspective of a poststructuralist school of thought, which links language, subjectivity, social organization and power in the study of phenomena, I use the case study approach as a means to explore whether integration of Indigenous thinking in western academic frameworks is a viable option. Jamie Baxter argues that "case study research involves the study of a single instance or a small number of instances of a phenomenon in order to explore in-depth nuances of the phenomenon and the contextual influences on and explanations of that phenomenon" (Baxter in Hay 2010 81). In that vein, it would be more rigorous to examine in detail an exemplification of a phenomenon. The 
body of Indigenous knowledge is vast and diversified, and would be extremely difficult to test in this context. WC was actively engaged in environmental stewardship. Further, with his Circle of All Nations work, he engaged beyond Indigenous partners to advance his objectives and work. It is recognized that WC commenced his project under earlier historical circumstances, that he launched his intensive decolonizing project in the mid nineteen forties, and that his objective was to mobilize and animate others to respect his Mother Earth. The proposed hybrid research approach, then, need not constrain emerging Indigenous scholarship that challenges location within a contemporary western paradigm. However, an analysis of his approach through a case study could help contribute to or advance such investigations.

Flyvbjerg examines the power of examples, and argues that "a discipline without a large number of thoroughly executed case studies is a discipline without systematic production of exemplars, and a discipline without exemplars is an ineffective one. (Further, he adds), (i)n social sciences, especially those that find themselves to be weak, more good case studies could help remedy this situation” (Flyvbjerg 2011 87). Can Flyvbjerg's call for exemplars to strengthen the discipline of social science be broadened to include Indigenous examples? I query whether the relational, integrative Indigenous approach can contribute to reconciliation of segmentation of the physical and human in studies in geography.

I use the example of WC's extensive environmental work and archival materials to justify this case study. An examination of his discourse is required and this includes all facets of his outreach, not just the written words. The evidence available for such a case study is extensive. The Circle of All Nations discourse is animated and co-created with a diverse range of players. It appears to cohere around some inherent integrative principle, and six years after his death, evidence of its presence may still be observed in ongoing activities and cyber- 
space communications. In my research, I study its past manifestations in textual/photo/video/audio archival material and examine contemporary evidence illustrative of any continuing emergent interplay of multiple and diverse actors and actants in this discourse.

Contemporary reference to his work and legacy will lead to conclusions about the possible integration of the William Commanda approach to knowledge generation on environment and relational issues within the academy.

\subsection{Structural Parameters}

\subsubsection{Geography, Cartography, Environment and Mother Earth}

Geography, derived from the Greek word, geographia: earth description, studies the lands, features, inhabitants and phenomena of the earth. Modern geography, while rooted in the work of geographical societies, expansion, colonialism and imperialism (Holt-Jenson 2008) is an all-encompassing and continuously developing discipline that seeks to understand the earth and its human and natural dimensions, dynamics and complexities. Geographers study spatial and temporal nature, and the distribution of phenomena, features, processes, and interactions, animate and inanimate, of the world we live in. Geography has typically been divided into two main branches, physical and human geography. Physical geography examines the natural environment and how organisms, climate, soil water and landforms produce and interact; it aims to understand the lithosphere, hydrosphere, atmosphere, pedosphere and biosphere (global flora and fauna patterns). Human geography is focused on how humans create, view, manage and influence space and environment; it also examines dynamics emerging from spaces that shape human society, including human, political, cultural, social, psychological and economic dimensions, relationships, patterns and processes. Historically, there were some schools of thought that emphasized linkages 
between the two domains.

Since the late sixties, with heightening awareness of pollution and excessive and uncontrolled exploitation of the resources of the earth, and their impacts, the environment leaped into the domain. Today, the field of Geography incorporates and comprises each of the time located preoccupations of the centuries: cartography, physical geography, human geography, environmental studies and emerging cybercartographies of the digital age; and now GeoHumanities and GeoPolitical publications examine links between spaces, places, humanities and politics.

WC's word for environment was Mother Earth: Kitchi Jojo Aki, expressive of her power, creativity, provisional capacity and responsibility for all; the critical thing for him was the nurturing of relationship with the primal and penultimate life-force. I came to realize that every aspect of his work was tied into awakening others to this understanding of environment, mindful as he was that in the colonized realities of his homeland, the newcomers did not possess this natural connection to his Mother. In this sense then, his thinking about environment is also a reflection of organic connection and emergent evolving relationship, as compared to notion of environment, as external the conditions which can determine modifications in the development of organic life.

WC embodied human as totally emergent of a cosmic and global world, and projected this ontology and epistemology on the local, national and global stage in continuums of fluidity, as well as in multiple-point-in-time eruptions. I liken these to volcanic flows that create new ground and nurture life, for example with birds laying eggs in hot ashes. Here, life is generated and nurtured in environments that are labeled hostile by others. It was in a parallel violent dance with the ice and rock that life originally evolved in the watershed of the Mighty Kichisippi, and this approach is part of his syntropic project. 
But an understanding of William Commanda's earth-based thinking and its relevance to academic research in Geography necessitates a grasp of the web of knowledge informing his ideology and comparing this with the philosophy that informed other approaches.

At the commencement of the Elizabethan Era, 1558 (sandwiched between the battles between the Protestants and Catholics, and Parliament and the Monarchy, and economically underpinned by Atlantic trade and slavery, and the time of Shakespeare (1564 1616), philosophy was moving from a spiritual to a wholly materialist conception of the world. I argue that when virtue and morals became associated with philosophy, the earliest expressions of western philosophy deviated from geographically grounded earth description that is consistent with the earth-based web of knowledge upon which WC's approach to environment and related matters is grounded. I have noted that, from a WC perspective, there are limitations to the scope of ethically motivated environmental efforts, no matter how well intentioned or helpful they might be; the required paradigmatic shift for him is the recognition and embracing of relationship with Earth; even more, emergence from Earth.

Entrenchment as human animal transforms the playing field, says Ian McCallum, who positions his findings as ecological intelligence (McCallum, 2008), and who introduces this dimension of thought into philosophical contemplations, following his immersion into the lives of the San Indigenous Peoples of Southern Africa. This dimension of thought challenges notions of religiously inspired dominion over earth.

The thoughts and conclusions of philosophers have had profound effects on the global stage over the past five hundred years. The history of social, political and technological change is inextricably bound to the history of thought. The Indigenous voice has been decimated, marginalized and rendered invisible over these past centuries, yet it persists, despite the minuscule nature of its presence at this time. William Commanda strove 
to inject his Indigenous reflections on the profound questions of life on the world stage in his lifetime of effort, striving to reach not just the specialists, but also everyman/woman/child. He wished to generate the desire and capacity for such earth based philosophical inquiry that he deemed indispensable to the individual and communal journey through life, a journey consistent with the Laws of Nature. The earth-based underpinnings of his thoughts informed his outreach and his global eco-community, the Circle of All Nations. The thesis examines its ongoing relevance and presence, six years after his death in his articulation of Ginawaydagunuc - All is Related.

\subsubsection{The Foundational Concept: Ginawaydaganuc All is Related}

William Commanda's approach to knowledge is predominantly empirical, grounded around the concept of relationality. Immediately, challenges present with the concept of empirical. I note that the dictionary associates empirical with observation and experience, and also with the idea of quackery. Thus, it compares unfavourably with a theoretic approach to research based on the prior development of hypotheses, with suitable experimentation to lead to scientific conclusions. However, when you approach the concept of Ginawaydaganuc as a Law of Nature, the theories that underpin it can be observed, tested and proven. This is what I explore in WC's empirical and experiential process of knowledge generation.

I use the foundational idea of Ginawadaganuc, All is Related, as the central earthemergent $\operatorname{cog}$ around which to assess each of four logics of inquiry to probe WC's knowledge. I use the associated methods and tools of the Circle of All Nations conceptual medicine wheel to structure my research, and to critically examine and justify its potential use as a scholarly exemplar. Its epistemological potential for bridging with the academy is explored by examining the assemblages of elements in the empirically grounded discourse. 
Over the past two decades, over the course of countless journeys and constant exposure to oral and written materials, I have become attuned to the very sophisticated and contemporarily relevant nature of WC's spatial and temporal knowledge and thinking. I have learned that the speech of oral traditions is quite different from the spoken language of literary traditions, and that multiple elements besides words constitute language and meaning. William Commanda's map is integrated in his very definition of himself as Mamiwinini, or Nomad. Fundamental to this naming of self is the practice being in motion mindfully, experientially and empirically.

Thus, for him, spatial understanding was determined by and emergent from his name for his people, Mamininini - Nomads. These were the people who were in motion, and who moved with great regularity; motionality was inextricably intermeshed with the temporal. $\mathrm{He}$ lived over the course of a century of tremendous transition from the industrial age to the information age, and his spatial understanding incorporated elements of environmental knowledge, journey and transformation of self and others on the land. In this sense, then, his mapping was not merely traditional with associations of primitive performance mapping; in fact it resembles and reflects the emergent mapping of Turnbull's understanding of the cyberworld (Turnbull 2007).

Our imagistic spatial/temporal explorations reveal parallels with key Indigenous words and ideas pertaining to cosmic, evolutionary, ancestral, bio-diverse, trickster (chance/uncertainty) and motional themes in the production of his Indigenous Law of Nature discourse. Knowledge creation was sensory, experiential, experimental, observational, collaborative, cognitive and generative, and emergent from time/space, movement, language and the Laws of Nature.

A joke that William Commanda liked to share can serve to transition us to an 
analysis of the empirical in his discourse.

The tourist asked the guide, do you know where we are? No, said the guide. Well, you are no good, said the tourist. Well, I am not lost! replied the guide.

\subsubsection{Syntropy - An Emerging Concept to complement an Ancient One, Ginawaydaganuc}

I introduce two new conceptual ideas that are gaining attention in Western academic thought, to throw light on the Law of Nature foundational knowledge base of Indigenous peoples. They are linked with entropy and ideas about time, causality, and energy: syntropy and negentropy. These concepts are relevant to the discussion of each of the four logics of inquiry.

I happened upon the term syntropy quite serendipitously. No physicist or mathematician myself, I set about to understand this earth grounded concept, to ascertain whether it would shed any light on the William Commanda discourse. Understanding syntropy helped me draw together key threads emanating from the multiple levels of WC's discourse. Some elements were inaccessible to me initially in my inability to understand his vast language. But they were rendered visible nonetheless, via this new Law of Nature lens. Syntropy resonates with everything WC tried to explain to me about his, and even more particularly, his father Alonzo Commanda's, conscious and informed Law of Nature knowledge acquisition, epistemology, ontological and ontogenetic approach to life lived as cosmic being. The Saturday Evening Post writer John Durant described Alonzo Commanda as The Best Guide in the World in his December 23, 1944 article with good reason.

I draw on the work of Ulisse Di Corpo and Antonelli Vannini, to explain the basic parameters. Their book Origins of Life (Di Corpo, Vannini 2011) and several video presentations explain the concept of syntropy. In his innovative research, which commenced in the 1970s, Ulisse Di Corpo examined the compelling geo-question about causality and our

universe, and introduced the concepts of retrocausality and supercausality. I suggest that Di 
Corpo tracks how western science was gradually acquiring the knowledge that WC's ancestors had already secured, as they learned how to live in the American North East as the Wisconsin Glacier retreated and during the post glacial period. This foundational knowledge was forcibly eradicated by vicious practices of colonization, conquest, and germ warfare, with near genocide, stifling of movement, destruction of relationship with land, food source, earth-based spirituality, imposition of religions, destruction of social structures, elimination of language and associated ways of being Indigenous, as well as by sexual exploitation and introduction of disease and alcohol.

In the following discussion, drawing on Di Corpo's timeline research into the scientific investigation of this phenomenon, I provide a brief resume of syntropy to provide a conceptual understanding of WC's epistemological project.

In the language of physics, within in our solar system, radiation and heat (thermo dynamics) from the sun to the earth transmit energy in the form of light to bring life into this our (closed) system; this becomes a paradox as living systems are not closed. Entropy, the second law of thermo dynamics, affirms that everything in the universe tends towards the dissipation of energy, randomness and chaos, till at some point everything in the system is at the temperature of the surrounding space, with no life generating thermo dynamic.

Causality is linked with the concept of entropy and the tendency of energy to dissipate; at the same time, properties of life persist in evidence of energy concentration, increase in differentiation, increase in complexity and growth of structures; this introduces the critical concept of retrocausality. Retrocausality is linked with the concept of syntropy, the tendency to converge, not dissipate. Effects propagate backwards in time consistent with the properties of life. Energy, then, is derived from causality as well as from retrocausality, and its effects propagate forwards and backwards.

Now, syntropy is not available in the macro level in which we live but is available in the quantum, sub-atomic level; life is the process of the extracting of syntropy from the quantum level and making it available in the macro. What allows one to see syntropy and its move from the quantum to macro level is presented in a particular law of the universe: the one at play in a hydrogen atom of a water molecule. Water is a critical living entity, and quite different from other liquids. According to the language of syntropy, water absorbs syntropy from the quantum level and materializes it at the macro level. Water shows cohesive and absorbing properties which defy the law of entropy, and shows growth of structures and complexities. 
Thus, the critical law of the universe, a Law of Nature, the law of syntropy, can only be seen operational in the presence of water: it affirms retrocausality, the future to past cause of effects because it demonstrates life force energy in its complex, cohesive structure, quite different from the entropic energetic dissipation of past to future, cause to effect, causality.

The opposite of entropy is the operation of negentropy within living systems; this is a temporary condition in which certain things are hotter than the surrounding space; they also become more highly organized in structure and function; these are the dynamics of living systems. Life is linked to negentropy because this dynamic takes things that have less order, like dead food, and turns them into things with more order, like cells in the body, tissues and organs, at the same time generating heat. Drawing on the entropy dissipation, negentropy activates life-generating processes. With respect to systems, within a vast perceived closed universe system, the solar system functions as an open system, with dual processes operational within a vast time span - what is sometimes called deep time. The syntropic movement activates negentropy.

There are three levels of time: causal time characterizes diverging systems (e.g., in an expanding universe, cause precedes effects, entropy increases, and time moves forward from past to future; with retrocausal time, energy converges, effects precede causes, entropy diminishes, but syntropy increases and time moves backward from future to past; supercausal time characterizes systems balanced between diverging and converging force, as with atoms: in these systems, causality and retrocausality coexist and time is unitary - past, present and future co-exist (Thumbadoo 2017 Personal Journal).

This is a complex discussion and videos on line can strengthen understanding of this flow of logic. I theorize now that the metaphoric language WC often used was indicative of this syntropic Law of Nature knowledge acquired by his ancestors over centuries of wrestling for life in the shifting waters and rocks of the post-glacial world. While the knowledges of all Indigenous peoples were irrevocably changed by colonization and assimilation, the wampum prophecy heritage, language, and his father Alonzo Commanda's detailed knowledge of the stars, seasons, waterways, fire and survival, relearned in the most exacting conditions of the natural and colonized world, left clues about this knowledge in WC's storytelling and language. Further, he reiterated the critical importance of water repeatedly; he could see the fundamental and critical Law of Nature itself under unceasing and destructive assault by the colonial will. The language of syntropy, negentropy, causality and retrocausality enabled me 
to understand this more deeply. I call this animation; this negentropic animation is what WC abstracted from the Laws of Nature and applied to, reiterated and made integral to his discourse and Circle of All Nations work.

I suggest that Indigenous Peoples had their own words for this phenomenon. I apply this syntropic lens in alignment with Ginawaydaganuc to interpret each of the parts of the thesis.

\subsubsection{Pentimento}

In addition to the cyclical flow of the thesis, each of the segments may be layered one upon each other, conveying depth in a pentimento fashion, where bottommost layers retain energetic presence and agency comparable to the ones on top. Bridging cultural divides is extremely challenging and crucially important in a globalized reality. One needs to be concise and succinct to express complexity, and sometimes it can be helpful to use one word to express that complexity and then to deconstruct it. Pentimento is an Italian word that refers to a visible trace of earlier paintings beneath a layer or layers of paint on a canvas. It is the reemergence an older image that has been painted over. Its literal meaning (intriguingly) is 'repentance' (from the Italian, pentire, to repent, derived from Latin paenitere, to regret). An understanding of pentimento can contribute to an understanding of the vital presence of past in Indigenous oral knowledge structure and communications; this is an obvious challenge for many non-Indigenous thinkers. The pentimento idiom has been animated repeatedly in Circle of All Nations peace building workshops (and articulated, for example, as the FROG principle, in our book, Learning from a Kindergarten Dropout (Thumbadoo 2005 57-67). Both William Commanda and I also engaged with systemic constellation work, and this field of exploration reveals resonance, reverberation, layering and relationship in zones or systems of difference or conflict. Thus, in pentimento fashion, 
for example, one can see a layering of colonization leading to land and resource depletion, to water contamination and to health crises, with each of the preceding actions still performing and exercising agency. At this point, suffice it to present the concept of pentimento as layered visualization of the four segmented components of the Circle of All Nations medicine wheel conceptual tool. Further, while each component complements and builds on the others, each also holds an integrity that can function autonomously.

\subsubsection{Storytelling}

I employ both a storytelling approach and theoretical analysis to ensure an authentic exposition of William Commanda's singular discourse, and the objectives and technology of his constructed bridging approach, in animating his epistemology.

An evolutionary, distinctly human, capacity for storytelling has re-presented itself on the global stage in a massive social explosion. Digital technologies are magnifying its reach, upsetting dominant paradigms and knowledge bases. In outpourings of volcanic proportion, storytelling is creating new ground for the presentation and performance of an infinite number of joint or networked dormant and emergent narratives.

It is intriguing to note that some interesting episodic frames in the human evolutionary story appeared on the global screen in 1981, with the epic movie Quest for Fire. It commences at the campfire, where land and territory are pitched together in the quest for fire for survival at physical and metaphorical levels. The movie includes graphic presentation of critical sensory acuity in the fight for survival in the contextualized and expansive zones of space and place, time and seasons. It hypothesizes the move from gesture and sound-making to language evolution and examines threshold, physical and negotiated relationship interface with elements of non-human nature. It portrays an evolving emotional repertoire of sensory and ultra sensory hyper-attentiveness. Fear, fight, violence, 
rage, othering, humour, patience and love are sequenced against a cosmic calendar, and a backdrop of rock, cave, mountain, waterfall, tree, moss, fog, quicksand, and animal, underpinned by movement and deep-seated cartographic knowledge. The movie concludes with Human the Storyteller. S/he has journeyed, circled across earth, and is back at the campfire, at a new stage in an ever-spiraling evolutionary epic, storytelling the experience.

This was followed by a new age notion of convergence in 1987, and interestingly, Indigenous presence entered the global stage with National Geographic videos of the songline territorial map imprinting of the Aborigines of Australia; Maori approaches to gesturing (nose rubbing); and African storytelling and healing templates presented by African writers like Credo Mutwa (Indaba My Children 1964, The Song of the Stars 1966, Isilwani The Animal 1996) and Malidoma Patrice Some (Of Water and the Spirit 1995, The Healing Wisdom of Africa 1999). This also included the restorative and sustainable relationship-based environmental stewardship priorities of the North American Indigenous Peoples, as voiced through William Commanda Algonquin, and his colleagues Martin Gashwaseoma Hopi, Martin Martinez Navejo, Peter Ocheese Cree, Albert Lightning, Joe Cupp Ojibway, Arvol Looking Horse Lakota, Phil Lane Jr. Lakota, Don Alexandro Mayan, Hum Butz Man Mayan, Kogi Shaman from Columbia, and countless others from across North America. I met most of these elders, and learned from them and their stories myself. I suggest that it is critically important to note these projections beyond colonial cartographic representation, and to acknowledge and understand the reassertion of multilayered temporal and spatial dimensionality in inter-connection grounded in earth-emergent ideological convictions. Its growing presence on the global stage attests to its impetus in alignment with escalating concurrent earth change processes. 
A growing body of neurological and cognitive research aligns the storytelling trajectory with the evolution of the human being and suggests that the brain is hardwired to storytelling. The research also suggest the brain is hardwired to movement.

In Research is Ceremony: Indigenous Research Methods (2008), Shawn Wilson discusses relationality, respect, ceremony, reciprocity and storytelling and Indigenous research paradigms. He references Tafoya on storytelling: “Stories go in circles. They don't go in straight lines. It helps if you listen in circles because there are stories inside and between stories, and finding your way through them is as easy and as hard as finding your way home. Part of the finding is getting lost, and when you are lost you start to open up and listen" (Tafoya, 1995, in Wilson 2008 17). Wilson argues that "Stories and metaphors are often used in Indigenous societies as teaching tools. Stories allow listeners to draw their own conclusions and to gain life lessons from a more personal perspective. By getting away from abstractions and rules, stories allow us to see others' life experiences through our own eyes. This information may then be internalized in a way that is difficult for abstract discussions to achieve" (Wilson 2008 17). Further, he adds that "When listeners know where the storyteller is coming from and how the story fits into the storyteller's life, it makes the absorption of the knowledge that much easier" (Wilson 2008 32).

Wilson also introduces us to Tafaya's principle of uncertainty: that it is not possible to know both the context and definition of an idea at the same time. The closer your get to defining or explaining an idea, the more it loses its context. At the same time, the more the context of an idea is explained, the further you get from its definition or focus.... It is only through realizing both the context and content/analysis that you will reach a deeper understanding of what the chapter is all about (Tafoya, 1995 in Wilson 2008 99). This is the essence of relationality (Wilson 2008). Thus, Wilson goes on later, with respect to 
ceremonial knowledge, Elders would be "comparing information from the dream work, and they'd start to realize that the various symbols were being dictated to them from a different part of their being. So, we're starting to see something more. And suddenly they'd come up with an answer. 'Well that's what we're missing because there's a certain thing being hidden here" "(Wilson 2008 113). These thoughts raise the flags about the incommensurability of knowledges, and the need for bridging mechanisms to create spaces for meaningful exchange.

Repeatedly, WC expressed great pain and frustration that Indigenous views on environment, (what he asserted as his Mother Earth on fire, on the proverbial hell fire of destruction), were either victim to colonialism, industrialization, greed, deceit and ignorance, were appropriated, misrepresented or dismissed, or grounded in erroneous/alternative science, and thus not fully understood. He hosted gatherings on the land to remedy this, and facilitate the exchange of ideas.

I add this background information, before we leave the storytelling lens into the research. I commenced working in correctional system in the seventies, a new graduate of literature, and an avid journal writer. Storytelling emerged as my most powerful tool in the counseling and case management of federal offenders in parole and in maximum and minimum-security prisons across the country over a twenty-five year period. It was my most effective strategy to optimize rehabilitation and public safety and security. When I met WC, it was in the context of my work organizing Aboriginal justice conferences, where we relied heavily on oral storytelling and talking circles to advance cross-cultural understanding, bridge-building, information sharing and relational change. The recent Truth and Reconciliation Commission work in Canada utilized similar storytelling to retrace history. Storytelling was also a technology used by WC in his own work. It became a critical part of 
our Circle of All Nations work, in writing as well as in gatherings and events. The past, present and emergent are now evident in today's rich and complex digital storytelling field. In many respects, this contains elements of oral storytelling, both frozen and emergent, and this is a dynamic field that warrants serious exploration with respect to its role in WC's environmental project. 


\section{Chapter 3 \\ Methodological Tool Kit}

\subsection{Overview}

\subsubsection{The Undergrowth}

I address language, philosophies, constraints, complexities, accommodation and opportunities in this cross-cultural, trans-cultural conversation briefly to situate the Methodological Tool Kit for this thesis. It is of the critical importance to be attuned to philosophical parameters and linguistic nuances in any examination of Indigenous ways from both the non-Indigenous and Indigenous starting points. It is an exploration that can

only be ventured upon with great care and respect, because the trajectory to bridging ways of knowing and understanding is potentially fraught with significant challenges.

Geographical research, like research in other disciplines, is based on philosophical assumptions or choices: "Philosophy is to research as grammar is to language ... just as we cannot speak a language without certain grammatical rules, so we cannot conduct a successful piece of research without making certain philosophical choices," says Elspeth Graham, in Methods in Human Geography, edited by Flowerdew and Martin (2005 10).

Thus, considering the parameters of any philosophical framework is essential to determining the most viable research design. In addition, the researcher must learn the appropriate vocabulary, terms, grammar and syntax of the community being studied. As Aitkin and Valentine note (2012), there are systems of meaning embedded in language, which require a serious researcher to engage in the associated cultures and practices: the more we know about the philosophical underpinnings the better we appreciate how influential they are to the research work. Further, different schools of thought have language rules that may make them incommensurable with others. There are challenging overlaps, 
complexities and interplay in this philosophical smorgasbord, particularly in research on Indigenous issues. It is a passage that can only be ventured upon with great care, because fundamental differences are potentially so daunting.

Viewed through a postcolonial lens, the discipline of Geography and study of nature emerges from Anthropocentricism (human-centeredness). This orientation is contrary to William Commanda's perspective as First Person on Turtle Island (North America) and to his understanding of and use of the term Law of Nature. His views emerged from his knowledge of the natural world: the cosmos, the four elements (fire, earth, water and air), the rocks and minerals, plants and animals, acquired through a range of observational, experiential, experimental, collaborative and spiritual/ritual/energetic techniques. He considered this Indigenous science.

The Collins Westminster Dictionary (1965) notes that science is the systematic knowledge of natural or physical phenomena; truth ascertained by observation, experiment and induction; ordered arrangement of facts known under classes or heads; theoretical knowledge as distinguished from practical; knowledge of principles and rules of invention, construction, mechanism, etc. as distinguished from art. Now, here, language and meaning present interpretation dilemmas because WC appropriates the words law and science; but he does not intend this to imply acceptance of the limitations of the positivist rule-based scientific method. Rather, it is to establish that his knowledge of nature and its rules of operation are grounded in evidence secured via extensive observation, experiment, experience, collection of data, collaboration and corroboration; also complemented by other ways of knowing; and acquired, accumulated and held individually and collectively. The point about wholistic knowledge being held by all is critical: Indigenous nature-based knowledge was critical to survival and was held and integrated by all, and not subject to 
social segmentation and the dualistic/ binary thinking that evolved elsewhere. This then takes the search for a philosophical framework in bridging cultural barriers to the domain of hermeneutics and interpretation. Here, flexibility and creativity as well as a dismantling of the dominant discourse in the accessing of a common language has to be ventured, even in the search for the tools for adequate research design.

Respect for the environment implies a philosophical value-based position and places discussion in an ethics rubric. Environment, according to the New Webster Encyclopedic Dictionary of the English Language (1971) means act of surrounding; state of being environed; that which environs; environ comes from Old French, vironner, to environ and virer, to veer: to surround, encompass or encircle; to hem in; to involve; to envelop. The Collins Westminster Dictionary (1965) adds these explanations: to invest; environment is explained as the external conditions which determined modifications in the development of organic life.

This inherent meaning of environment was fundamentally different from William Commanda's understanding of the earth. His understanding emerged from his language. Algonquin is a land-based language, wherein the land itself, its sounds, its physical, geological and biological realities (rocks, stone, animal, plant, the elements) evolved with the language. William Commanda's word for environment was Mother Earth: Kitchi Jojo Aki, expressive of her power, creativity, provisional capacity and responsibility for all; the critical thing for him was the nurturing of relationship, personal and familial relationship, with the penultimate life-force. I came to realize that every aspect of his work was tied into awakening others to this understanding of environment, mindful as he was that in the colonized realities of his homeland, the newcomers did not possess a natural connection with his Mother Earth. In this sense, then, his thinking about environment was a reflection of organic bio-diverse 
interconnection and emergent evolving relationship, as compared to environment explained above as the external conditions and surroundings that determined modifications in the development of organic life. Having drawn attention to this distinction, please note that, for the purposes of this thesis, the use of the word environment will be associated with its common use in environmental studies in the context of environmental challenges and stewardship.

Kitchin and Tate (2000) note that geography means different things to different peoples in different times and different places. They quote the Geography Working Group's Interim Report (1990):

- Geography explores the relationship between the earth and its peoples through the study of place, space, and environment. Geographers ask questions where and what; also how and why.

- The study of place seeks to describe and understand not only the location of the physical and human features not only of the Earth, but also the processes, systems, and interrelationships that create or influence those features.

- The study of space seeks to explore the relationships between places and patterns of activities arising from the use people make of the physical settings where they live and work.

- The study of the environment embraces both its physical and human dimensions. Thus it addresses the resources, sometimes scarce and fragile, that the Earth provides and on which all life depends; the impact on those resources of human activities; and wider social, economic, political and cultural consequences of the interrelationship between the two (Kitchin and Tate 2000 4).

While noting that defining geography is fraught with difficulties, they identify human geography as the study of society in relation to space and place, and segregate physical geography. They present critical rationale to substantiate this approach to research: the study of people and human-made objects requires different research techniques from the study of natural phenomena; further, there are clear philosophical and methodological differences between human and physical geography research (Kitchin and Tate 2000). However, the items listed under physical geography - biogeography, climatology, geomorphology, hydrology, meteorology, quaternary environments, soils were precisely the items that 
preoccupied William Commanda in his understanding of the Law of Nature, and with the recent attention to environment crises attest to their profound relevance to the postcolonial study of society in relation to space and place. Researchers have recently begun to call ours the age of the Anthropocene, in view of human impact on environment and its physicality; this has been an urgent concern of WC's since his childhood, and throughout his adult life. It is only by straddling the domains of physical and human geography that one can situate William Commanda's approach to the study of the environment in the context of theoretical approaches.

Is this at all viable, given the multiple cultural divides? The credibility of the research is dependent on identifying and locating it within a viable philosophical framework, and identifying and applying appropriate methodological tools to conduct an analysis of his epistemology and practices.

\subsubsection{The Methodological Approach}

I am examining the contemporary relevance of the legacy of Indigenous Elder William Commanda, grounded in his earth-based Law of Nature point of reference. I probe for evidence that indicates that William Commanda created his Circle of All Nations global eco-peace community as a bridging mechanism into his Indigenous worldview. I argue that this work evolves around his constant prayer, statement and performative exhortation, Ginawaydaganuc - We Are All Connected, All is Related, with Earth animate, inanimate and unseen forces, and with each other, beyond the realm and scope of the Anthropocene. I theorize that the research offers opportunities for integration and knowledge generation in academic discourse on related eco-peace themes. I also argue that such integration is of critical importance in contemporary times of accelerating climate change, extreme weather and global conflict. 
The research explores the integration of this relational approach to environmental thinking in the academic world. I use the foundational idea of Ginawaydaganuc, All is Related, as the central cog around which to assess each of the respective logics of inquiry, associated methodologies and the tools of the Circle of All Nations conceptual medicine wheel, both to structure my research, and to critically examine and justify its potential use as a scholarly exemplar. Its potential for bridging with the western academy will be explored by exercising meticulous rigour in probing the assemblages of elements in the empirically grounded discourse, using key contemporary methodological tools, including storytelling, documentaries, text and photographs to examine William Commanda's human and nonhuman community, employing reflexivity and NVIVO for data analysis of the core themes, frequency, coherence and refractions of the discourse, and deploying the cybercartographic atlas and social media to track themes of relationality, fragmentation, regeneration and emergence across space and time.

The specific methods associated with the four proposed logics of inquiry or entry points into the research of performance mapping, historical/dialectical, phenomenology, and cybercartography, are respectively, geo-narrative, narrative, auto-ethnography and critical reflexivity, and cybercartographic digital atlas/social media analysis.

It has been challenging to reduce examination of WC's approach to environment, in view of its scope and interrelatedness. I considered a variety of theoretical approaches, including critical, postcolonial, decolonizing and Indigenous platforms, but determined they would limit access to his discourse. With each of the approaches selected, I considered a range of methodologies, and eliminated many with illuminatory potential, in order to select tools from his repertoire that would intersect with academic thinking and could contribute to integration on environmental issues and pedagogy. It might have been useful to examine in 
detail one aspect of his work, for example the Asinabka Sacred Chandiere Site challenge (www.asinabka.com), since it embodies rich material for human geography research with issues of contemporary neo-colonial, neo-liberal corporatization, decolonialism and Indigenizing, settler/ally relationality, power and control, creation of truths and truth effects at the country's central geopolitical site playing out against an unprecedented and intractable trajectorial backdrop of Indigeneity in the forefront of daily government, national media and social media. However, I determined that such a focus would have limited the understanding of WC's approach. The contemporary land-based struggle, where corporate power and the establishment continues to be challenged by multiple grass roots fires is a David and Goliath story, and is in part a manifestation of the vitality and reach of the $\mathrm{WC}$ discourse six years after his death. The thesis is focused on amplification of his approach to accelerate possible impact on the intractable geopolitical environmental challenges.

I also undertook an extensive review of western philosophy to grapple with the research question. While it is not included in the thesis write-up, it contributed deeply to my understanding of WC's approach. Likewise, my personal exploration of literature and photography are informal methodological tools that informed the analysis. Discussing metaphoric earth writing in geopoetic research terms, Springer advocates for the "undisciplining of geography to open up theoretical space for voice, where a material space of emancipation might follow" (Springer 2017 1), and reflects on de Certeau's challenge of perceived correct ways to do geography, noting that "the parallels with colonialism are hard to ignore, as its gaze constitutes a strategy of embodying disciplinary mechanisms wherein power is relayed through various nodes that legitimize particular ways of seeing and doing (de Certeau 2000 in Springer 2017 5), and he points to "making meaningful changes through the more hands-on approach of scholar-activism where theory and practice 
converge as praxis (Chatterton 2008 in Springer 2017 8), and where "committing human geography to a progressive, inclusive, and emancipatory agenda necessitates a negation of the ivory tower and the false dichotomy between the academy as a space of knowledge production and the wider society as the domain of social" (Pickerill 2008 in Springer 2017 9). My photographs of WC's work of over the years constitute another powerful investigative tool that has informed the research profoundly, and could constitute a WC Thesis in Photographs', but is minimally reflected in this thesis.

In addition to the academic research, I have examined and reflected critically on the extensive archives, books and documentaries in WC's archives. Further, after his death, Circle of All Nations colleague Sunbow (Iron Wood Log Project) compiled a listing of on-line references and audio/visual clips on WC. A separate bibliography of a portion of WC's personal library and the Sunbow's list are included in the appendix, but it is noted that not all materials may be readily accessible since this includes old materials, and many grass roots video capture and documentation. They attest to WC's presence in the cyber world over time and space, and are included to illustrate the nature of his discursive reach. Since some of the grass roots material may not be available in academic journals, links to on-line references are included to illustrate key points. Specific Circle of All Nations materials have been examined in some depth as they reveal and expose WC's methodology and tools (e.g. through wampum and canoe). In addition to the extensive documentation since 2000 in the websites www.circleofallnations.ca and www.asinabka.com, they include the following books and documentaries:

The Indian Crafts of William and Mary Commanda David Gidmark Grandfather William Commanda A Few Reflections on the Very Special Occasion of your Ninetieth Birthday 2003 Circle of All Nations Colleagues Learning from a Kindergarten Dropout 2005 R.V. Thumbadoo Learning from a Kindergarten Dropout Book Two 2008 R.V. Thumbadoo Passionate Waters Butterfly Kisses Kayak Photo Journal 2008 R.V. Thumbadoo 
William Commanda Biography in Up the Gatineau Vol 40 historical publication, 2014

Ojijkwanong Encounter with an Algonquin Sage, Lucie Ouimet 2002 NFB documentary Circle of All Nations Gathering 2001 documentary

Good Enough for Two canoe making documentary, 2003 Valerie Pouyanne, Loon Productions

The Awakening, 2007 Jeremy Wright

Mishomis William Commanda, 2008 Ironwood Log Project SunBow

Portage of Wisdom, 2009 Patrick Gravel

Inner Healing Indigenous Trees of Wisdom, 2014 Adrien Esposito documentary

In addition, since 2009, Circle of All Nations has also been a presence in social media via several Facebook pages:

William Commanda

William Commanda Legacy Asinabka

Circle of All Nations

Romola's Photo Page Nature CAN TEACH

\subsection{The Four Logics of Inquiry}

The Circle of All Nations' four direction conceptual medicine wheel process operant in WC's performative and processual mapping work moves from inspiration/ignition to activation/animation to reflection/reciprocity to articulation/representation, to spiral again in an emergent, evolving non-static fashion. This presents interesting avenues for further exploration of his power, knowledge, truth and truth effects discourse in the context of Mamiwinini/Nomad movement. With a deeper understanding and appreciation of this Indigenous web of knowledge approach, could a case study of the work, thoughts and influence of WC, and his geo-narrative, inform typical questions of research - who, where, when, what, why, how and whether - and support an understanding of the relevance of WC's knowledge generating base within the western academy? 


\subsubsection{Conceptual Medicine Wheel Orientation Map}

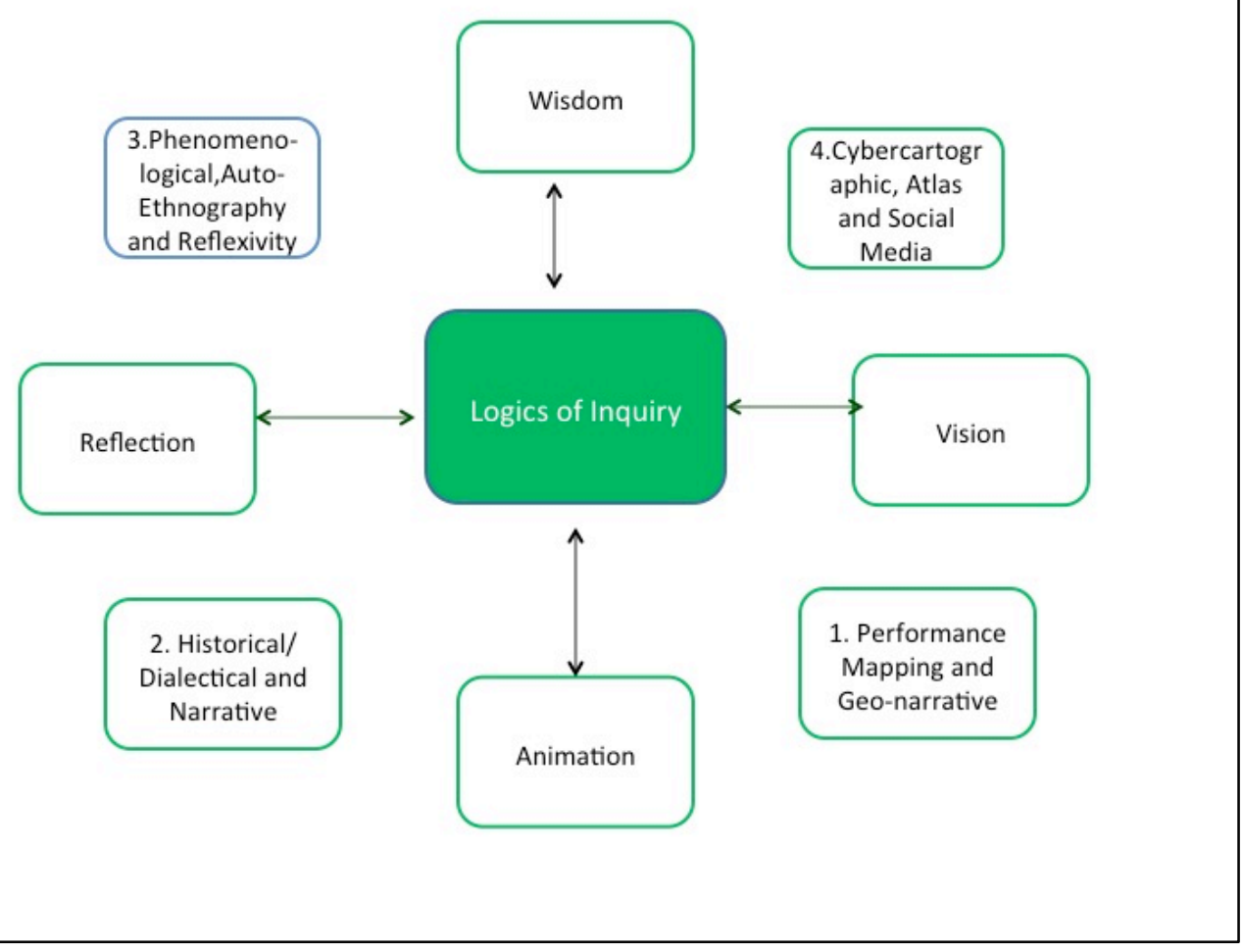

Figure 3. Conceptual Map of Circle of All Nations Logics of Inquiry

\subsubsection{Performance Mapping, and the Geo-narrative Ideological Foundation}

In this section of the thesis, I explore critical aspects of WC's ways of knowing and web of knowledge. This ideographic and ideological background was activated by intense relational interface with the Ottawa River Watershed and the American North East, and informed his ontological way of being as well as his ontogenetic relationship with nature and people and was inscribed in motionality. In William Commanda's case, ideas are incorporated in his language and his name for his people, Mamiwinini - in its basic translation, nomads. We moved all the time, he said. The name itself and the language are the living map. WC re-presented and re-vitalized many components of that heritage, not just for himself, but also for countless others globally, Indigenous and non-Indigenous. These included people from diverse races, cultures, religions, languages, ages, education, occupation, social classes and economics. He ignited for them a relational reconnection with Earth. This was premised on the perpetual articulation and sharing of his prayer Ginawaydaganuc: All is Related, shattering entrenched discourses of duality, difference and separation, as well as Anthropocentric relations with the natural world. 
He animated his worldview through such means (tools, technology and technicity) as

a) an ancient epistemology (living oral learning and teaching processual mechanism) in the language of the Sacred Wampum Shell Belts, ancient spiritual/sacred artifacts and mnemonic devices reconciling the invisible/intangible and material, prophecy and history, the temporal and spatial, infused with agency, and offering direction for environmental and social intercourse;

b) gatherings and relationship-building events with and amongst diverse peoples, privately and publicly, with new technologies of non-verbal communications, formats for communication like talking circles, and textuality;

c) ceremonial, ritual, art and creativity activities, including drumming, singing and dancing, to ignite a legacy of performance (versus material) mapping; and

d) land-based activities fed by the womb of earth herself, to ignite relationships, respect and responsibility to the natural world.

\subsubsection{Historical/Dialectical Approach and Narrative Exploration}

WC consciously engaged in a massive decolonizing and indigenizing project from the mid nineteen forties onwards, before such words or concepts were examined by the academy. The extensive reach of WC's effort can be likened in a small way to Spivak's Can the Subaltern speak? (1988), and A critique of postcolonial reason (1999); Said's Orientalism (1978) and othering, and Fanon's The Wretched of the Earth. He himself quoted Wise and Deloria, and The Red Man in the New World Drama (1971) and this represents his determined counter-discourse to the establishment. WC's own copy of the latter book includes this handwritten quotation by Deloria: “Our ideas will overcome your ideas. We are going to cut the country's whole value system to shreds. It isn't important that there are only 500,000 of us Indians ... What is important is that we have a superior way of life. We Indians will show this country how to act human. Someday this country will revise its constitution, its laws, in terms of human beings instead of property. If Red Power is to be a power in this country it is because it is ideological ... What is the ultimate value of a man's life? that is the question. 1971". His interrogations of the geo-political reached activists and environmentalists first, and are only now entering the academy. In view of WC's prioritization of Mother Earth and the Laws of Nature, I examine the geo-political parameters of his historical narrative, and point to 
opportunity to access the storytelling for further academic analysis and abstraction. Indigenous scholars are now breaking new ground in research, and their emerging and influential work is already impacting academic thinking; some are beginning to study WC. Future research on WC will engage with this evolving body of provocative academic literature.

As noted, extensive collections of textual, archival material, books, audio and video recordings, photographs and artifacts (e.g. canoes) are available for analysis. Four shifting narratives shaping WC's story are of particular interest:

a) Maudit sauvage/Dirty Indian - the historical presentation of the First Peoples of the continent, created by the doctrines of colonization and imperialism, now increasingly challenged and discredited; the digital age, internet, Wikipedia and social media are accelerating the retelling of history;

b) Canoe builder Respected for what I can do with my hands - here the particular discourse begins in 1981 with the building of a canoe in Roskilde, Denmark, and the meeting with Queen Margarethe. I also examine other factors contributing to a new representation of the Indigenous in the Nation's consciousness, as a force to be acknowledged;

c) Noble Savage - the new age revival of the image of the noble savage, produced in the vein of "othering" (Said 1978), presents a romanticized overview delimiting the discourse; but WC's Lament, in the vein of George Grant, rejects this confinement; and d) Commanda Virtuoso Performer - the fourth discourse presents his animation of his own life and work, as one grounded in his relationship with his Mother Earth, and with earth-based ceremonial, spiritual, heritage traditions and associated knowledge acquisition and knowledge generation practices, broadening and animating this further in the co-created William Commanda/Circle of All Nations discourse.

\subsubsection{Phenomenology, and Auto-Ethnography and Critical Reflexivity}

Here, I adopt an interpretive phenomenological approach to this research into the William Commanda discourse, with focalization from his, my and other critical reflective engagement on relevant data. Auto-ethnography is a qualitative method involving the explicit writing of an embodied and situated self into the research. It often entails writing in literary fashion, involving autobiographical narratives, wherein the author/researcher actively reflects on personal choices, emotions and knowledges as a vital part of the construction of 
the research. As I reviewed videos, formal and informal written materials, both WC's and mine, I realized the extensive process of auto-ethnology and critical reflexivity that had created his own discourse; hence, I give primary place to his reflective voice here. However, I also incorporate my own reflexivity into the analysis, and note my own positionality. I have engaged in journaling since the late seventies, and this practice of reflection informed my work with WC, and our joint storytelling. My own voice occupies a crucial platform in this research project, given my engagement with the subject matter, and both critical reflexivity and auto-ethnography can serve as rich qualitative research tools to guide this work. In this context, I iterate that

a), my earlier academic studies fell in the domain of literature, and while not expressed in the language of discourse analysis in the seventies, do in fact, reflect an active engagement in this approach; I have also written three journal designed storytelling books. They have included photographs and images. This graphic-literary background lends itself to operationalization in my current research project; b) critical reflexivity is a process of constant, self-conscious scrutiny of the self as researcher and of the research project. As Dowling notes, "being reflexive means analyzing your own situation as if it were something you were studying. What is happening? What social relations are being enacted? Are they influencing data?" (Dowling in Hay 2010 31). The exhortation to examine ideas and thoughts about the research process, its social context, one's role in it, outsider/insider attributes (inclusive of socio/economic/education/employment, racial, ethnic, religious, and gender differences in my case), positionality, partiality, contextuality, assumptions, power relations, in particular asymmetrical ones, are critical to credible research and intellectual honesty;

c) Dowling also notes that subjectivity involves the insertion of personal opinions and characteristics into research practice and that this is relevant and potentially enriching because qualitative research methods involve social interaction - thus, rapport, communication skills and awareness of the specific social context, semiotics and issues help to decipher text and discourse. Further, d), inter-subjectivity plays out in the meanings and interpretations of the worlds created, confirmed, or disconfirmed as a result of interactions (language and actions) with other peoples within (a range of) specific contexts (Dowling in Hay 2010 35); in the case of the Circle of All Nations, there is a vast range of inter-subjective moments for analysis; in bridging the cross-cultural world, I bring a particular and irreplaceable capacity for reflexivity, given my unique history and relationship with the work over twenty years. 


\subsubsection{Cybercartography, and the Cybercartographic Digital Atlas and Social Media}

Cybercartography is the modern theoretical approach selected to examine the ongoing presence and relevance of WC in contemporary times. The term cybercartography was introduced in 1997 (Taylor 1997) to describe the transformative innovations that were taking place in the domain of cybercartography by the late nineties. He defined cybercartography formally as "the organization, presentation, analysis and communication of spatially referenced information on a wide variety of topics of interest and use to society in an interactive, dynamic, multimedia, multi-sensory format with the use of multimedia and multimodal interfaces", with seven major elements: multimedia, using vision, hearing, touch and eventually smell and taste (Taylor, Pyne 2009 3). Its use demands interactivity; online spaces are cyber spaces for a new kind of mapping. They incorporate some of the multi level processual and performative components of Indigenous mapping. The importance of narrative/storytelling/oral discourse to Indigenous peoples impacted the development of the innovative Nunaliit digital atlas platform by the Geomatics and Cartographic Research Centre (GCRC) at Carleton University (www.gcrc.carleton.ca D. R. Fraser Taylor, Director), registering the shift from a publishing/presentation model in mapping to a participation/processual model, and permitting multiple narratives (Crampton 2001). Circle of All Nations has expressed this as we make the road by walking it. Cybercartography presents tools to explore the Indigenous mapping experience as a multi-dimensional/multileveled/multi-pronged, vibrant, cognitive and sensory interface, much more closely approximating the rhythms and rules of nature, as incorporated in the Indigenous animation of mapping.

The cybercartographic digital atlas and social media, in particular Facebook, constitute key technical and processual tools to interrogate WC timeless moccasin-telegraph 
performative mapping. The specific approach involves a time-based lens scrutinizing aspects of his discourse over the course of his 98 years, and then its ongoing presence since his death in 2011, in particular his assertion of intangible, sacred relationship with land in his Circle of All Nations Asinabka/Sacred Chaudiere Site Legacy Vision. Circle of All Nations has managed several Facebook pages to animate William Commanda's discourse since 2009.

The cyber tools permit a new means to scrutinize the ongoing relevance of the Commanda approach to environment, and related matters, such as

a) land based language and symbolic imagery (petroglyphs, pictographs, canoe motifs);

b) William Commanda's use of the Circle of All Nations as a zone of encounter and permeability to address incommensurability;

c) photographic reflection on the intangible yet compelling voice of space and place, giving Laws of Nature their esoteric presence in academic study; and

d) contemporary social media engagement with William Commanda and his ideas.

Each of these theoretical entry points and the associated methodological tools are contextualized and examined in much greater detail in Chapters Four, Five, Six and Seven, in order to optimize entry into the singular and complex discourse of William Commanda, and to examine the bridging pedagogical framework of his teaching style.

Each of Chapters Four, Five, Six and Seven include (1) contextual orientation into the logic of inquiry, (2) presentation of the theoretical frame, methodology and tools, (3) conceptual medicine wheel orientation map, (4) discussion, analysis and observations regarding the WC narrative discourse, (5) concluding remarks, (6) summary of findings, and (7) a William Commanda exemplar of the logic of inquiry.

\subsection{Data Analysis}

William Commanda was popularly known as the techno elder. Computer-assisted qualitative data analysis software (CAQDAS) are now presenting researchers with both new tools for data analysis as well as innovative ways to think about data analysis. As Peace and 
Van Hoven note in the paper entitled Computers, Qualitative Data, and Geographic Research, geographers have already been employing computer-based tools for statistical analysis, modeling, graphics, cartography, image processing, visual imagery, and remote sensing. Now geographers and other social scientists are looking to computer support for analyzing unstructured qualitative data, and "the range of geographical research activities has been extended to include word searching, data coding, data storage and retrieval, memoing, graphic mapping, hierarchical tree-building, concept building, and reflexive report-writing (Peace and Van Hoven in Hay, 2010 297). A wide range of voices is represented in the Circle of All Nations web sites, archival blogs, photo files and current face-book pages. The associated material constitutes rich data for examination and analysis of multiple perspectives on its discourse, beyond that of William Commanda, as virtuoso, and myself, as researcher. I engage in preliminary use of Nvivo research software in this research project. I suggest that this qualitative research tool can serve to examine this Anicinabe approach to knowledge generation on environment, relationships and related issues and reveal its potential for integration in academic research; it also informs and complements the discourse analysis approach. 


\section{Chapter 4 \\ Theoretical Lens: Performance Mapping and Geo-Narrative Mamiwinini Trail Marking - and Syntropic Agency of the Seven Fires Prophecy Wampum Belt}

\subsection{Orientation into the Performance Mapping Logic of Inquiry Lens}

This chapter explores the geonarrative roots of WC's performance mapping, a nonmaterial expression of spatial relationality. I examine four foundational influences that informed his ideological worldview, (i) a spiritual dimension, (ii) language, (iii) river, rock and land, and (iv) creative expression. These preliminary ideas are provided as an entry into the web of knowledge that informed his interpretation of the Laws of Nature. As Casti notes, "It has ... become evident that each society produces particular views of its territory, according to the specific relationship established with it and the practices it is invested with... Scholars agree that symbolic apparatus used to represent the world derives from the values on which a given society is based, values according to which societal knowledge will be organized..." (Casti 2014).

William Commanda and his ancestors state that they have lived on Turtle Island, the continent of North America, since time immemorial. It is significant that the physical map of the continent reveals the shape of Turtle. How did the tribes that spanned the vast contintent know this and name her so? How did they know that the massive landmass was an island, surrounded by water from sea to sea to sea? It is interesting that Turtle lives in the ocean but spawns on the land, bridging domains. Turtle energy is important to Indigenous Peoples, and is a critical symbolic element in the Circle of All Nations work, books and in the Seven Fires Prophecy journey narrative.

With the retreat of the Wisconsin Glacier, WC's ancient ancestors, the Paleo Indians, 
moved into the American North East, leaving artifactual and mythic evidence of their presence on the land during the last twelve thousand years. These ancient peoples were hunters, as evidenced in their stone tools, and they followed the animals that followed the plants that evolved in land newly released from ice. They studied the geographical and cosmological features of the natural world and they registered detailed knowledge of a range of geo-cosmic space/time realities in markers that confirm such knowledge. WC believed that this thread of knowledge remained alive with his direct ancestors and that language was one of the repositories and transmitters of this knowledge. The language of the people of the Ottawa River Watershed forms the generic root of the Algonquian/Algonkian dialects of the eight four nations that span the continent. As Hessel notes, the "Algonkins of the Ottawa River Watershed form the linguistic prototype ... of the Algonkian family of languages" (Hessel 1987 2).

WC speculated about the sources of the knowledge of his people, realizing in the evidence of his language, and their orality, that they had had significant geo-cosmic knowledge, the threads to which had been ruptured by colonization. He retained the motional pathway to this knowledge throughout his life in part by being in perpetual motion. As a child, he moved regularly between the reserve and bush and his family's hunting and trapping grounds, as a guide, he travelled further around the province of Quebec, then during his working life he travelled extensively across the Atlantic Provinces and Eastern Seaboard of the United States, and later he travelled globally. In this, he, unlike many other Indigenous Peoples, was not confined to the reserve, residential schools or other imprisoning borders. WC was also an artist and craftsman. "Works of art and the artistic process are ... central to the interpretation and understanding of a given way of life" (Vastokas 1973 2) and serve much like the underlying rules of grammatical structures in 
language, to help make sense of ideographic world views; thus this capacity contributed to his knowledge.

Motional spatial understanding was determined by and emergent from the name of WC's people, Mamiwinini, and his identity was commensurate with the idea of map in motion. Nomad is a limited translation of the word. WC's temporal and spatial understanding incorporated elemental and motional Law of Nature knowledge. His performance mapping was embedded in and emergent from orality and narrative. WC's storytelling mapping included his wampum heritage and the Seven Fires Prophecy. The migration motif of the prophecy resembles and articulates the epic and odesseyan. The prophecy traces the journey of his peoples over time and space, as charted in metaphor and physicality, and it also articulates connectivity at multiple levels, including with turtle shaped islands. This is consistent with Turnbull argument that "What nearly every culture seems to share, in one form or another, is the recognition and celebration of the hodological or topokinetic in their mythologies, ontologies or epistemologies, and especially in their stories, songs and maps. This commonality is based in the connectivity of trails - the creation of meaning through marking and linking” (Turnbull 2001 143).

I extrapolated information about the cosmic spatial/temporal knowledge of WC and his ancestors myself from my examination of his language and stories about his father. With respect to time, the three primary cosmic bodies, Moon, Sun and Earth are named as time markers in Algonquin and their names reveal the profound temporal knowledge of the people from ancient times. WC's Algonquin word for Moon is Tibi Kizis; Sun is Kitchi Tibi Kizis. Moon demonstrates the smaller monthly time cycles in her being, thirteen of which constitute the big time cycle. Sun controls the big yearly time cycle. Sun takes identity from Moon and is the Big Moon. It is Moon's magnetic connection with Earth that renders interconnection with cosmic most significant, and it also informs the twenty-four hour time cycle. Earth manages the twenty-four hour cycle; Night was Tibikut, and Day, Kijikut. There are further refinements in the temporal knowledge, which simultaneously demonstrates cosmic spatial knowledge. For example, in the vast sea of stars, Enogosuk, some are the winter stars, Adawomok, only visible during specific months of the year. WC's own name is linked with the planet, and reveals the ancient knowledge of the differences between planets and stars. 
Likewise, constellations were studied, named and embedded in legends. ${ }^{1}$

The naming of the continent Turtle Island is a semiotic attestation of both spatial/temporal knowledge and of the relational associations of Turtle and Moon. As noted, Turtle is a powerful symbol that most tribes across North America use to associate with their homeland, and the image is included in rock art across the continent. Turtle's shell markings align relationally with the traditional Algonquin calendar of thirteen Moon cycles each year, and approximates the days of a moon cycle. Moon's magnetic powers impact Earth's magnetic core, the force of gravity, tidal movement, spawning of fish, plant growth cycles, and the migrational cycles of many creatures, including the Turtle. For a people whose lives were charted by water, from the time of the retreat of the Wisconsin glacier and the formation of the Great Lakes and rivers, the Moon was a most significant cosmic entity. 2

Ginawaydaganuc is the term used by WC to discuss the interconnection of all things in his world. It incorporates a vast conceptual notion that serves as a template for understanding person-to-person connection and interrelatedness, with associated implications of inextricable emeshment. However, it also embraces relationship with the animate and inanimate geo-cosmic world at multiple levels, and thus it provides the

\footnotetext{
${ }^{1}$ I just learned that WC's daughter, Evelyn Commanda Dewache, has come to notice the winter stars herself, through intense observation (Personal Conversation, October 4, 2017). She realized that the night sky was different than it had been during the summer. This is most fascinating to me, since I have never heard her articulate this during the past twenty years that I have known her. Yet as a child, she was both raised in Algonquin, the language that registers geo-cosmic knowledge, and she was acutely aware of her grandfather's (Alonzo Commanda's) preoccupation with stars; now, in her seventies, she was reclaiming direct access to her ancestors' ways of knowledge acquisition and knowledge generation. She had obviously been observing the night skies for some time; but she was suddenly noting the significance of its reality in an epiphanic manner. I noted that my accessing of the same knowledge was acquired differently. Some of the knowledge of her ancestors is only being retrieved, understood and acknowledged now, for example, as revealed in the documentary Before the Lake was Champlain (Timrick 2005), and in CBC's 2005 series of documentaries on geology, where Indigenous knowledge held in stories and myth is now being reported. In fact, this Indigenous knowledge has lead modern science to hypothesis and verification processes now made possible by technology.

2 The ancient Anasazi Peoples of the American Mid West created the structures at Chaco Canyon. A research group entitled the Solstice Project discovered the solar solstice marked in a petroglyph at Pueblo Bonito. The Hopi informed them that this was a critically important feature. What is even more significant is that at Chaco Canyon, buildings are also aligned to the Moon's nineteen year cycle, as it circuits closer and then farther away from Earth every nine and a half months. This is the only architecture in the world that reflects such knowledge of the lunar movement. These people visited the Algonquins, according to WC's friend Bernice Wagoush McGregor, with stories passed down describing them as the Ancient Ones (Thumbadoo/Commanda Unpublished Joint Journal Notes, 2007). Moon is also associated with a woman's twenty eight day menstrual cycle. WC himself was give a platform stone pipe retrieved from the Ohio River: it has 28 lines etched along the rim. These interrelated issues, and especially the interconnection with the cosmic, was of tremendous interest to WC.
} 
conceptual entry into his performance mapping and geo-narrative.

While material maps were originally seen as mirror geo-reflections and then later as socially constructed, the introduction of the concept of performance mapping opened a door into a new world of non-western mapping. In 1988, Woodward and Lewis noted that a performance may take the form of a non-material oral, visual, or kinesthetic social act, such as a gesture, ritual, chant, procession, dance, poem, story, or an ephemeral demonstration such as a drawing or model in the sand, or other means of expression or communication and have the primary purpose of defining or explaining spatial knowledge or practice (Woodward and Lewis 1988 4). Harley demonstrated in Deconstructing the Map (Harley 1989), that the Eurocentric practice of material mapping failed to incorporate the spatial, performative and ethereal understanding of the peoples of traditional societies. The articulation of performance mapping permitted a legitimization of Indigenous pathways to knowledge in cartography.

\subsection{Theoretical Frame, Methodology and Tools: Performance Mapping Geo-narrative}

The following examination of academic thinking in contemporary cartography over the last twenty-five years sets the parameters for the focusing of this theoretical lens on an examination of WC's distinctly Indigenous cosmic web of cartographic knowledge, his performance mapping orientation and the land-based geo-narrative. This discussion follows the specific western mapping trail that points to possible integration with WC's understanding of land. I highlight insights that contextualize, permit and inform crosscultural relational bridging into this Indigenous knowledge stream.

Harley is the pivotal geographer who drew on poststructural thinking to challenge to the Eurocentric presentation of maps as scientific and objective. He examines the 
implications of the efforts of the British Cartographic Society to reframe cartography as the "science and technology of analyzing and interpreting geographic relationships, and communicating the results by means of maps" (Harley 1989 2). Integrating these ideas with Foucault's work on texts (discourse, knowledge and power, and the inherent rules of engagement) and Derrida's on textuality (particularly his discussion of metaphor and rhetoric), Harley embarks on a search for social forces that have structured cartography, seeking to locate the presence of power and its effects in map knowledge. He finds that western maps are sanitized of art/creativity, because they are grounded in measurements and standardization and are therefore deemed to present reality untainted by social factors. But the social rules of mapping, including the invisible ones, are influenced by other rules of cultural production, including social hierarchy. These inform the map that "hides and denies its social dimensions at the same time as it legitimates" it (Harley 1989 7). He also notes that text is not limited to word, and, as such then, maps are graphic cartographical text. Thus, Harley deconstructs maps, both re-inscribing and re-situating meanings, events and objects within broader movements and structures, and seeking out the blind spots, the margins, the inter-textual zones, where the text involuntarily reveals tensions between what it means to say and what it is constrained to mean.

Harley also examines two representations of power in cartography, external power located in the individuals/institutions that commission mapping and exert power over it, and internal power emanating from the creation of the map. Here, Harley is incorporating the Foucauldian position on the omnipresence of power, which comes from everywhere and which the cartographer channels into the map as knowledge or information. The map serves both as the arbiter of the power and as an embedded social vision. Thus, he observes that "context is stripped away, and place is no longer important. On the other hand, the maps 
reveal the ambivalence of all stereotypes. Their silences are also inscribed on the page: where, on the page, is the variety of nature, where is the history of the landscape, and where is the space-time of human experience in such anonymized maps?” (Harley 1989 14).

Harley's footnoted reference to Roszak, outside his formal paper, elaborates on the idea of the anonymized map: "We forfeit the whole value of a map if we forget that it is not the landscape itself or anything remotely like an exhaustive depiction of it. If we do forget, we grow rigid as a robot obeying a computer program; we lose the intelligent plasticity and intuitive judgment that every wayfarer must preserve. We may then know the map in fine detail, but our knowledge will be purely academic, inexperienced, shallow" (Roszak in Harley 1989 19).

Harley's deconstructive analysis presented the pivotal platform not only for interrogating the European map but for exposing the type of power inscribed in its structure and released from the mapping process. It also refocused the lens for peering anew at the mapping of Indigenous peoples. During the depression years, WC worked for a logging company, using his dog team to move food for loggers from campsite to campsite; he observed the use of compasses and other equipment to survey and then slash his territory. It was a harsh, aggressive, invasive and indifferent style of mapping land. Even though colonization and the reserve boundaries had severely restricted the movement of his peoples, still he retained the relational knowledge of place finding. (His father was known to see and travel in the dark, and Highway 317 in Quebec is known informally as the Gabriel Commanda trail, after his uncle). Initially, when he pointed to boundaries created by beaver engineering while we drove the highways following the Ottawa River, I could not see this demarcation of nature that was so visible to him. After the 2013 flooding in Calgary, when the Bow River reclaimed its nature-determined pathway, I understood his mapping much 
better. In colloquial Indigenous language today, it is said that eight rivers returned to their beds with the 2017 flooding in the Ottawa River Watershed. I also realize now that it was that manner of approaching and understanding the voice of the land that WC wanted to teach me when he insisted on getting me a kayak. The observational, experiential and empirical door into the contextual was Nature's instructive pathway to deep knowledge of her features and laws.

Rundstrom introduces two interesting insights into non-western maps. One is the incorporation of cultural practice in oral communications. These incorporate the intangible. The other is the employment of performance-based modes to transmit meaningful information. The actions produced are more meaningful than any objects produced. Inscription focused cultures (western) hold and fix meaningful information, typically through objects (Rundstrom 1991).

Rundstrom discusses process cartography as comprising two concentric ideas, situating the map artifact within the map-making process, and placing the entire map-making process within the context of intra-cultural and inter-cultural dialogues occurring over a much longer span of time. The processual view, he posits, makes cartography less exclusive because it is open ended and dynamic, with meaning contingent on process, and where acts (or action) empower artifacts (Rundstrom 1991). These are critical insights for understanding Indigenous mapping. Both body and mind are in constant motion and inform the knowledge generation. Process is more important than product, and therefore honed in for evolving. The dominance of verbs in WC's speech, and the regular use of the present tense reinforce the perpetually alert anticipatory knowing predilection. These also suggest the anticipatory alignment with cybercartography. This is of course quite different from the dominating power of Google Maps that inscribe and control pathways to places today. I 
learned how unfit WC's mapping training had rendered me for use of Google Maps during a 2013 road trip; while I had never gotten lost in over thirty decades of driving across the continent, I found that I was getting lost constantly with the prescriptive Google instructions.

Kitchen and Dodge also note that Harley's analysis advanced the postmodern deconstruction of the map. Then they analyse Crampton's focus on the Foucauldian observation that that there is no escaping the entangling of power/knowledge. Crampton challenges the ontological premise inherent to the foundational view that the world can be objectively and fully mapped using scientific techniques that capture and display spatial information. What he focuses on is the being of maps, how maps are conceptually framed to make sense of the world, rather than the contingent and relational products of a history of cartography. Kitchen and Dodge also examine the work of Pickles; he sees the ontological foundation as not simply representing but producing territory. Thus for both Crampton and Pickles, the being ontology is the predominant feature of cartography. Having reviewed this, Kitchin and Dodge identify the ontological insecurity of this analysis of cartography (Kitchen and Dodge 2007 335) and point to its ontogenetic nature, manifest in embodied social and technical practices that are always remade every time they are engaged with, and are also transitory, fleeting, contingent, relational and context-dependent. Ontological security, however, is maintained, they posit, because the underpinning knowledge (recognizing, communicating, interpreting, translating and communicating) is learned and constantly reaffirmed, is a co-constitutive production between inscription (map), individual and world, and is always in motion (Kitchen and Dodge 2007). They also introduce the concepts of transduction (explaining everyday life as sets of practices to solve ongoing relational problems) and technicity (the power of technologies to resolve the problems) to present maps as ontogenetic. Maps emerge through contingent, context-embedded practices 
to solve relational problems, constituting emergent cartography. Maps emerge through practices, and epistemological research focuses on the science of practices, not representations (Kitchen and Dodge 2007 342). The relational movement and process nature of WC's epistemology integrates this ontogenetic orientation at every turn, As well, it 'transducts' empirically, as is evident, for example, in his canoe teaching exemplar, restoring empiricism from 'quackery' in the re-newing Indigenous form of relational knowledge generation. It moves constantly between being and becoming, and furthermore, points to practice-based vehicles for addressing relational incommensurabilities. His canoe-making documentary Good Enough For Two (Pouyanne 2005) illustrates and demonstrates performative mapping extending its reach into the inter-personal and inter-cultural zones.

Pearce adopts a strong decolonizing lens to examine mapping from an Indigenous viewpoint, in Mapping Non-Western (2009). She notes that in western science, the map renders space observable, rational and malleable, presented "through use of the graticule as a spherical coordinate system and grid" (Pearce 2009 374). Thus, culturally constructed and epistemologically driven by the Eurocentric frame, the cartographer's gaze empowers sovereignty, domination and imperialism, and other means of mapping space are made invisible or dismissed as primitive. The cartographic encounter of colonization reveals the power dynamics at play, for example in the stifling of the movement and processual mapping of the Indigenous and the simultaneous dispossession of lands and resources. While this style of mapping still dominates, Pearce turns her examination to non-western mapping, and elaborates on the intrinsic features that are under study now through the lens of emergent mapping (Kitchin and Dodge 2007, Rundstrom 1991, 2009, Crampton, 2007). These include (a) the processual (dance, chant, radiating movement) and performative (ornamentation, dress, inscription, toponomy) versus archival and artifactual (Rundstrom 
1991); (b) boundary versus wayfinding multidimensional spatial awareness; and (c) maps as communication versus visualization. Pearce also notes connectivity between sacred and profane space, integrated via performative ritual, and in the cosmography of spiritual geography with the physical, political and cultural. Here, the coordinating system is radial, with variable and divergent centres, rather than the grid.

Rundstrom (2009) in Counter-Mapping, examines overtly political forces now engaged in the mapping discourse. Postcolonial counter-mapping is now challenging the hegemonic politics inherent to western maps, associated with land dispossession, resource abuse, social and population disruption. Here Indigenous and other marginalized voices challenge the hegemonic capacity to dominate and oppress through coercion, force and also through the tactic consent of those dominated. They also challenge the associated maps, and utilize modern technologies, GIS (geographic information systems, satellite images), GPS (global positioning systems) and the Internet, in this counter-mapping. While there are benefits in the communications, networking and mobilization opportunities of this mapping approach, Rundstrom also warns of dangers. A key one is the use of the language and tools of the dominant society. This can result in the reifying and distorting power of transforming something abstract into material reality, particularly in the case of Indigenous cartography, and of creating new realities based on alien ideas. Rundstrom points out that "mapping back at the state usually requires fixing boundaries and imposing stasis on the land which, in turn, overthrows the old ways and reinvents land use as a territorial claim that can provoke internal conflict" (Rundstrom 2009 317).

I suggest WC sidesteps this danger with adroitness. This is why when others talk about a treaty relationship with First Peoples in Canada, WC instead states that "I am at home, and all others are simply not ... unless they are invited in, they can never be at home." His 
ontological Circle of All Nations project, informed by his wampum heritage, to make the others his relatives, was motivated by four priorities: (a) to assert his rightful place, (b) to enable the others to become grounded in his multi-dimensional world, for them (c) to learn relational respect and responsibility to it, and (d) this, for their own 'well' be-ing. It is ironic to be writing this particular line as global leadership now creates a World Mental Health Day (October 10). In the context of the rains, floods, hurricanes and fires that have dominated and transformed the North American landscape (and beyond) relentlessly through 2017, WC would be noting the processual and performative activity of Mother Earth herself, and the inability of the others to dance, drum and (throat) sing in concert with her, in health and strength, as his ancestors had through her violent evolution over the past fifteen thousand years. He also sometimes despaired of the strengths of Indigenous peoples, sapped of motional lifeblood by confinement to reserves, diminished by reactional mapping back at the state, and by consorting with inscription and the bounded, and thus weakening themselves, with diminishing capacity for negentropic resurgence. Yet, at the same time, he said repeatedly that he was never without hope, aligned with the inexhaustible powers of the Laws of Nature.

From the time of his research in 1991, Rundstrom is already alert to some of dangers of the man-made tools of the state: he astutely notes that disenfranchised cultures may choose to move from being incorporating cultures to inscribed re-presentation, by tapping into the cartographical texts of the enfranchised (Rundstrom 2009). This, I suggest again, is something WC consistently refused to do, challenging national, provincial, reserve and interpersonal boundaries at every level, and going beyond land to challenge spatial/temporal construction at multiple other levels. He believed that life on earth, as we have known it, was at stake, and this fueled his passionate counter-mapping project till the day of his death. 
(There are several land-based struggles currently playing out in his homeland, including at the Asinabka Sacred Chaudiere Site; here it is interesting to take note of the number of his nonIndigenous colleagues now counter-mapping on his behalf).

Continuing in the pathway that Harley opened in research on the social construction of maps, Crampton examines maps as social construction from two perspectives, one to reveal power/knowledge practices in the Foucauldian manner and two, to explore geographical visualizations that reveal patterns. He acknowledges the epistemic lead provided by Arthur Robinson in 1952, examining maps as communication systems and maps as fields of power relations where knowledge is constructed, and goes on to consider two approaches, the Map Communication Model and Maps as Geographical Visualization (Crampton 2001). With the Map Communication Model, four critical features are noted: (a) separation between cartographer and map user; (b) the map is intermediary; (c) the map communicates information from cartographer to user; and (d) the ability of the map user to comprehend, learn and remember information communicated by the map is determined by cognitive and psychophysical capacity or indoctrination, that is awareness of and embeddedness in the force field.

Crampton posits Geographical Visualization as a questioning or sense-making activity, as compared to the Map Communication Model that is answer-delivering. He illustrates this point by drawing on the concept of cartography cubed (MacEachren and Taylor 1994): wherein traditional cartography emphasizes public use, low interactivity and revealing knowns, while visualization emphasizes private use, high interactivity and exploring unknowns.

Following Crampton's discussion of the Map Communication Model, I note that in the context of doctrinal religions, the force-field out of which the map emerges is the 
ideological backdrop created by the god-cartographer; the god-cartographer authorizes the map-priest; the map-priest instructs the map-user; the map user will get it and be responsive depending on how well he is indoctrinated in the ideological force field; so who is the godcartographer who creates the force-field? For example, the biblical creation story does not reflect accurate knowledge of the evolution of the earth. Who is the map-priest who interprets the force-field? The three dominant and related religions, Christianity (including Catholicism and its post 1500 s reactionary off-shoots of Protestantism, Calvinism, Presbyterianism, Puritanism, Lutheranism etc.) Islam and Judaism, are doctrinal. The godcartographer grants man dominion over earth and the map-priest interprets what this means. In WC's Indigenous world, the cosmic force-field was accessed directly by individuals and communities as cosmic beings themselves.

With spatial technology comes the integration of visualization, knowledge discovery in databases and distribution and dissemination via the internet. Distributed maps unlike static maps link information from various sources within a user-defined environment; the information may be present in many forms (maps, images, sounds, video and text) in a multimedia database, and its use demands interactivity. These online spaces are cyber spaces for a new kind of mapping. They incorporate some of the multi-level performative and processual components of Indigenous mapping. Poststructural analysis has deconstructed the power/knowledge contextuality of western colonial maps; and technology offers new mapping opportunities for the recovery of subjugated knowledges. Recent technologies permit new voices to enter the domain of mapping. Spatial and temporal relations are now vibrant and ever-emergent, and new ethical opportunities present (Crampton 2001).

Taylor et al are actively engaged in the creation of hybrid space via Indigenous/digital/geographic informative networks, developing the field of 
cybercartography in the innovative work of the Geomatics and Cartographic Research Centre (GCRC), Carleton University, and integrating critical academic perspectives with Indigenous and modern technology, through the creation of on-line inter-active maps and geo-narrative (Pyne and Taylor 2012). WC's discourse will, in part, be animated and examined using this technology in an interrogation of multi-dimensional digital space-time mapping. WC is also a vibrant presence on Facebook social media, and continues to assert and to challenge in his post bumus performance mapping. This would suggest that foundational ideology is critical to Indigenous mapping.

Methodological Tools

The specific methodological tools that have provided me with entry into WC's performance mapping thinking are drawn from multiple road-trips with him across the American North East and the Eastern Seaboard and throughout the Ottawa River Watershed. Our first major trip in 1998 took us from the heart of the country, the Chaudiere Falls, along the northern shores of Lake Huron, through Sault St. Marie, along Lake Michigan and south to Minneapolis/St. Paul, and Pipestone, Minnesota, then around the entirety of Lake Superior, and back to the capital city. It was an epic eight day journey. Thereafter we made a trip to Nova Scotia; several trips along the Eastern Seaboard to Cape Cod; several trips through the interior and along the shores of Lake Champlain into New York State; multiple trips along the North and South Shores of the St. Lawrence; and several trips following the Ottawa River, and countless trips between Kitigan Zibi and Ottawa. There were also trips into Algonquin Park, and the one to Giant Turtle Rock is discussed later in the thesis. These trips were undertaken throughout the year, in all seasons and in all kinds of weather. I both made notes and internalized the knowledge and information WC imparted, and we both complemented our knowledge with study of the land and waters 
through books and videos. I refer specifically to key Ottawa River books in the discussion. I note that WC also served as Honorary Chair of the Ottawa Heritage River Designation Committee, and that we had access to its extensive research. Documentaries on Chaco Canyon, Mayan Pyramids, The Mound People, calendar rocks of the America North East, and many others complemented our textual sources. WC studied documentaries and literature about the mapping practices of ancestral peoples. For example, he had materials regarding Nazca Lines, Ancient Navigators, Chariots of the Gods, Chaco Canyon, the Mound Builders, and the maritime navigators and marine hunters of the American North East, amongst others like the Vikings, in his library, and he also knew academics (Lee Pennington, Pennington Gallery, Ekstrom Library, University of Louisville; Dr. William Sullivan and Ken Leonard, Massachusetts) who had researched some of this material directly. He travelled across the North American continent for the Sunbow Five journey of 1995/96, and visited the Sun Pyramid of the Mayans during the Summer Solstice of 1996, in his search to retrieve such knowledge (Thumbadoo/Commanda Unpublished Joint Journal Notes and videos; McFadden 2006 http://www.8thfire.net/).

I probe language, semantics and semiotics to negotiate his ideological views. I include in our www.asinabka.com site a personalized geo-narrative of the Ottawa River Watershed, to draw the reader into critical elements of one map location that was of critical importance to WC. I incorporate tangible and intangible information in my analysis, and out of the geo-history, and I abstract elements pertaining to the rock and water history that reemerge in other areas of the WC discourse. I discuss the Vastokas book on the iconography of the Peterborough Petroglyph site, to process the performance mapping and reflexive knowledge generation practices of his ancestors. Together, these constitute part of the web of knowledge that informed his work and teachings. 
Over the course of his journey over the land, WC offered many prayers for the continent of North America. This included placing the sacred tobacco on the land and in the waters, as a conciliatory gesture to heal wounds inflicted on it, and also to acknowledge the presence of the ancestors and avert the looming environmental catastrophe he saw emergent from the historical period.

\subsection{Conceptual Medicine Wheel Orientation Map}

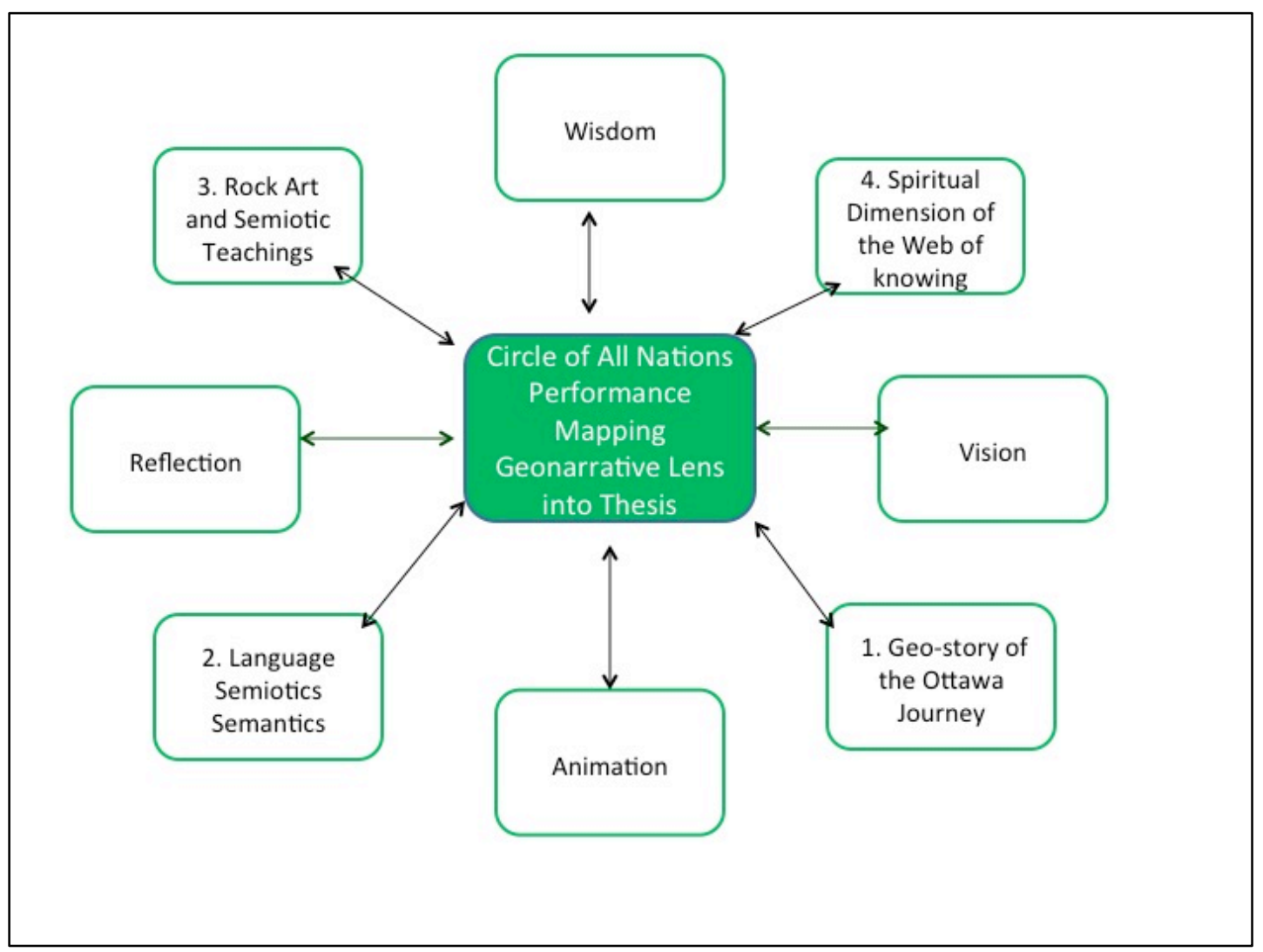

Figure 4: Conceptual map of Circle of All Nations Performance Mapping - Geonarrative Lens into Thesis

\subsection{Discussion, Analysis and Observations}

\subsubsection{Special Rock Features of the Ottawa River Valley and Algonquin Heritage}

(a priori - prior to experience; a posteriori - derived from experience; a fortiori - even 
more so; a fortiori arguments are strong arguments or premises from which all other arguments pertaining to it are derived).

WC and I drove along the Ottawa River many times, and he shared many stories about this river and watershed; this perhaps is the most important river of Canada. We complemented his knowledge and memories with formal research, and developed relevant Ottawa River geo-narrative, commencing with its ancient rock formation history.

The reports of modern day researchers (Kennedy (1970), Legget (1975), Donaldson and Morrison (in WC's Archives) acknowledge that the Ottawa River was an important pathway for First Nations inhabitants for many years. We are told that they utilized its geological resources for thousands of years, and were familiar with its geological character. Our notes attest to the fact that we studied the river repeatedly from multiple angles ourselves. Our detailed annotated document on the geological history of the Ottawa River Watershed is available at www.asinabka.com. The following material establishes the critical importance of four key rock sites over deep time, and gives voice to the geo-narrative of the watershed itself.

The Ottawa River is the only Canadian River that traverses the land of all four of earth's lithosphere creation time periods. The combination of rock and water is of importance at various places along the river, and this combination in part contributes to the special significance of certain areas to WC's peoples. These are associated with the idea of spiritual relationship or the sacred.

The Kichisippi is best known in all historical texts as the Grand River of Canada. It is birthed in Lake Capimichigama, 155 miles due north of Ottawa. That is obviously a very special orientation. The Kichisippi is the largest tributary of the St Lawrence (which is birthed in Lake Superior, the largest of the great fresh water lakes which features the spectacular Sleeping Giant Rock feature), and it is fed by several rivers from the north and south. The river flows from the north in a westerly direction, then turns south to broaden into Lake Timiskaming. Of interest in its passage is the Gowganda Strata Formation bordering Lake Timiskaming, part of an episode of continental glaciation (2.2 billion years ago) from before the last Ice Age, its geological history revealed by the Nipissing diabase igneous rock. This was known to the Algonquins in its topographical presentation on the west shore of Lake Timiskaming as Spirit Rock. WC told me that there was a peculiar way the water moved backwards in this area, and this knowledge was recorded in the Algonquin 
name for the waters. Further down the river, at the critically important Mattawa River, (that led to the interior of the country, and revealed the intimate knowledge of the transforming lands), the ancient people operated a red ochre mine, ochre being very important for their ceremonial and burial practices; note that the Paleo-Indians were known as the Red Paint People; this special quarry became known as Porte L'Enfer/Devil's Warehouse, its identity as a place for generating spiritual knowledge being annihilated by the priests. Ochre is used in burial and resuscitation ceremonies. As WC said to me, they tried in every way to make us forget these things.

The river continues south, and then swings east. Here, there is the Grenville formation, comprised of metamorphic and intrusive rock, presented most dramatically and graphically in Oiseau Rock, (acknowledged as the Eagle Rock site revered by the Algonquins, and marked by sacred paintings and legend) at the metamorphosed limestone (marble) rocks near Allumette, Morrison (a significant site of archaeological artifacts) and Calumet Islands, and Rocher Fendu. The Algonquins knew about uranium and the heavy water at Chalk River and the Joachim Rapids, and tried to keep the newcomers away from the area. Allumette Island is of historical significance and is acknowledged in the 1613 Champlain encounter with Algonquin Chief Tessauot; and was also sought as a reserved sanctuary by William Commanda own great, great grandfather, Pakinawatik, in his efforts to safeguard the ancestral rights of the Algonquin in the Ottawa River Watershed in the mid-1800s. This was another spiritual power place.

The other item of note is the fact that ten thousand years ago, there was a much larger flow of water, and the large glacial lakes of Ontario and the Prairie provinces and the upper Great Lakes all drained into the Ottawa River, and there are many underwater caves, channel and twisting passages under the river bed; (one well known dramatic and visible example is the Bonnechere Caves. WC had the opportunity to walk within the Bonnechere Caves and quite spontaneously, he felt called up to drum in this dark watery basin. It was a powerful reminder that First Peoples knew about both the features and geological transformation of their ancestral waterway, as well as of their spiritual knowledge generation and energetic and sonar relevance. This is consistent with the contemporary explorations of Sheldrakian morphology and the extension of mind over space and performance mapping.

There were two very special passages where the water flowed under the river floor, and the spectacular sounds made were deemed from the womb of the earth herself: these, together with the pounding of the rapids, which could be heard from two furlongs away, drew travellers to the Asinabka/Chaudiere.

Several Algonquin names remain in the area, despite the recent centuries of obliteration of Algonquin presence at the Chaudiere. The entire area is called Asinabka, the place of glare rock, created by the ancient sea floor of 460 million years ago. Asin is the most ancient name by which the people of the Ottawa River Watershed were known. Akikou (mistakenly identified Asticou in historical time) refers to the area around the falls, Akik, referring to the pail or bucket or cauldron shape of the Pautik - rapids or falls. The Akikpautik was the Chaudiere Rapids/Falls. The Algonquin name Akikojiwan referred to the rock caverns through which water 
flowed under the river floor, and rock, water, wind and current combined to express something spectacular in the voice of the Earth; the Ojiwan referred to the water passing through the rocks and the Akik, pointing to where this was happening. Recent place-naming at the Asinabka Sacred Chaudiere Site has created some confusion; however, all the names attest to the draw of dramatic geographical features of the site that were known to the ancient peoples for well over eight centuries. Recent uncovering of archaeological artifacts in the Jacques Cartier Park area of the Gatineau River, as it enters the Ottawa River in the capital city, affirms, for example, the presence of chert from Northern Quebec and New York State, and copper from Lake Superior. (The Vastokas Team concluded that the Algonkians of the watershed were closely aligned with the people north of Superior and its spectacular Sleeping Giant rock, and were Mamiwinini who did their annual cyclical rounds across their territory, as directed by the rhythm of season, water over rock, and the gifts of the earth (Sacred Art of the Algonquin A Study of the Peterborough Petroglyphs Vastokas, J.M. and R.K., 1973). Given the lengthy exploration of the land over time, the translation of Mamiwinini as nomad or traveller reveals its incapacity to express WC's identity adequately).

A friend of WC's, Evan Prichard, Algonquin writer and researcher (Native New Yorkers, 2007, Henry Hudson and the Algonquins of New York, 2009), undertook some unpublished research for WC on waterfalls and islands, and determined that Indigenous meeting places evolved where the sound, energy and power of waterfall, convergence of two or more rivers, and islands were present. State and provincial capitals across the United States and Canada have developed over these areas; for multiple reasons, including the specific features of the Mighty Kichisippi, and its circular waterfalls, one critically important meeting place was the Asinabka/Chaudiere Site, at what has become the capital city; further the area is marked by other significant water falls. The largest of the tributaries feeding Ottawa River are the Tenagatin, that is, the Gatineau, from the north; and the Rideau, from the south. These rivers, feeding into the Ottawa as it moves from west to east, create the form of a natural medicine wheel, centred on the spectacular Akikpantik Chaudiere Falls. Both these rivers feature their own spectacular waterfalls. The name Tenagatin alludes to its upwards elevation orientation, and to the once spectacular Pangan Falls, the "pipe" falls at Low, Quebec. While the name of the Rideau is obscured, it is noted that many French names were translations of Indigenous words, and it is known that Algonquins walked on the rock ledge lying under the Rideau/curtain falls overflow. The name for the Ottawa River Valley area of glare rock, Asinabka, reveals knowledge of the ancient sea of 460 million years ago in the acknowledgement of the bedrock, much like the Ojibway legends of the Great Falls beneath Lake Huron are now being confirmed by modern day sonar testing.

In the Hawkesbury area of the former Long Sault stretch, Legget writes that:

"The great rapids of earlier day did not freeze in winter, though their spray made beautiful ice patterns. Turbulence of this kind can cause the formation of an unusual form of needle-ice, generally known as Frazil ... The most unusual natural phenomena on the river, great ice humps that used to rise every winter almost forty feet above the normal river level in a narrow section just below Greece's Point, some 
seven miles downstream from Hawkesbury"... a narrow gorge in the river bed 301 feet deep and 1,500 feet long, was ... discovered; the slow passage of water through this had allowed the frazil, formed within the Long Sault Rapids, to accumulate beneath the ice cover, creating the humps which always attracted so much local interest" - this had been noted by Chevalier de Troyes in 1668 (Legget 1975 17). With the building of the Carillon Dam, the water level was raised, which destroyed this unusual feature for all time. Here then was the special power rock site on the river, in the approach to the convergence with the St. Lawrence; the area around Rigaud (near the Lake of Two Mountains Algonquin gathering area) was one of the most biodiverse in the region; the land passed from the Algonquins to the missionaries during the early centuries of colonization, and this knowledge was obscured.

As noted, the Ottawa River disappeared into two underwater channels at the area of the Chaudieres, symbolic of the connection with the womb of Mother Earth, a referent of great relevance to many Indigenous peoples. In colonial times, these became called devil's holes; and eventually were also reconfigured and sealed. This was an unpardonable assault on Mother Earth to WC, and in part it contributed to his call to see the Chaudiere Falls undammed to the extent possible. In the same manner of place naming, other sites of tremendous spiritual importance to the Algonquins were renamed for the diable, devil, associated with European notions of blackness and evil, and even the term Windigo acquired fearful, even horrific, overtones for some. (Thumbadoo/Commanda Unpublished Joint Journal Notes 2007).

It was not just the naming that drove Algonquins away in fear from spiritually significant places. People and especially women were attacked and brutalized along the rivers. What used to be places of connection and knowledge acquisition became abandoned. Late Algonquin Elder and language teacher, Bernice Wagoosh Morin McGreggor, told me of many such criminal practices, particularly along the Gatineau. Algonquin artist Janet Kaponician created a moving birch bark painting of one tragic story of the Asinabka Place of Glare Rock perpetrated during the construction on Parliament Hill. A young Algonquin girl was raped and murdered by workers on the hill in the early 1800s, and her body impaled on a tree to make her stand upright to make it seem like she was still alive to paddlers on the river, but her flowing hair, which would normally have been braided or tied back, alerted her people to the tragedy. The Highway of Tears does not only exist in British Columbia and in contemporary times; nor does it only exist in residential schools. It is etched across the 
country and stains the land and all people. At least, that is what WC believed, as he transitioned from intimate acquaintance with the obscured horrors of wars and colonization across North America, and anger over the near genocide of his peoples, to a pathway to peace, in his vision for a healing centre at the Asinabka Sacred Chaudiere Site. He strongly believed the land and its peoples needed energetic cleansing and healing.

This short narrative of the geography and history of the Ottawa River leads me now to these anecdotal points with respect to WC's epistemology centered on Ginawaydaganuc relationality.

One: The key features of this remarkable river, in its original form considered the second wildest river in North America, with the multiple waterfalls and rapids oxygenating the atmosphere, was intimately known to Algonquins of old, and in particular to WC's father, Alonzo Commanda and his knowledge of the river was integrated with the cosmic and stellar knowledge of his ancestors, and their knowledge of the Laws of Nature. This is evidenced in several reports by WC and others; Alonzo Commanda could tell to the day, when the waters in the lake would turn, during the spring and the summer, with warm waters rising and cold descending and vice versa, and with organic material shifting. After multiple years of trying to figure this out, I finally noted one clue, and photographed a particular type of mist bouncing off the lake surface one fall. Another story pertains to the directional flow of the headwaters of the river at Lake Capimichigama. The name translates to Traverse Lake, with the waters flowing in one direction and the winds in the other, and sometimes dangerously so. One winter, WC, a young boy, was trapping up north with his father. They were preparing to head home as the ice was breaking, and presenting a passage, when the wind whipped up, and conditions 
quickly became terrifying for crossing. WC recalls that his father had to remove him from the canoe, then paddle their goods across the wild waters, then come back for him, and do the journey across again. That was when he began to realize how skilled and strong a water man and paddler his father was. Recall that the landscape and weather were already being transformed by logging by this time, this impacting wind velocity and direction. Respect for his knowledge deepened one January, when in the mid-winter cold, his father told him that if he had any cut wood in the bush, he should move it out, because the area would be flooded on February 24 (that would have constituted a very early spring thaw). To the day, his father was right. The 2017 flooding throughout the Ottawa River Watershed immediately brought Grandpa Alonzo Commanda's prediction about the 1974 floods to the minds of WC's son-inlaw, Tommy Dewache, and his daughter, Evelyn Commanda Dewache, and Grandpa Alonzo's granddaughter Daisy Mary Commanda Jacko, separately (Personal Conversations, Spring 2017). In the fall, he had said, next June, the lake will be flooded and you will be going around in canoe. He died in March. In June, the historical 1974 flood came. WC's own log cabin meeting house was swept away and WC did in fact canoe where his home now stands. It is interesting that the dams upstream had contributed to the flooding. What are the implications of paying attention to this Indigenous way of understanding the Laws of Nature? The Government of Canada's website on Environment and Climate Change Canada notes the following: "The Gatineau River, a major tributary of the Ottawa, drains approximately 26000 square kilometers of the Gatineau Hills lying north of OttawaHull. It rises to an elevation of 490 meters above sea level and flows into the Ottawa River at Pointe-Gatineau. The river has been extensively developed for hydroelectric 
power and is also used in log driving operations. Between May 14 and July 2, 1974, the Gatineau River underwent its most serious flooding since the turn of the century".

Two: In Chapter 5, I discuss my kayak learning from nature experience on the land, consistent with the guidance of WC. At this point, I discuss how, during the spring of 2017, the Ottawa River itself alerted me to several features that contribute to my understanding of how Indigenous peoples would have communicated with the waters and land. First, one windy day in late April, I noticed the wild waves at the rapids at Bates Island, over which the Champlain Bridge runs. I have watched paddlers there many times, but this was the first time I noted the massive power of the wave that made it appear as though the river was flowing from east to west. I contemplated the force, then, with which such water would hit at impediments like dams, when additional rushing water would oblige it to follow its preordained path. In fact, within a few weeks, the river assaulted all the dams along its path. It caused unprecedented flooding and damage throughout the watershed. The most ironic one is the overflow at the contested Jacques Cartier Site, where, as noted earlier, artifacts at least 8,000 years old and from as far away as New York State and Lake Superior were uncovered in 2014. Indigenous challenges to protect the special area were not resolved and remain in court. It would appear that the river was weighing in on the side of the protectors of the Paleolithic site during the Spring of 2017.

In the Spring of 2017, I recorded another alert from the river: a strange noise from the bottom of the river, unlike anything I had heard before. I later learned that is was the rolling of rocks on the riverbed, being violently moved by some strong spring underwater current. I recalled remarks about turbidity currents, (noted in 
WC's Heritage River Designation Research documents, in William Commanda's Personal Archives): The wackes commonly display graded bedding indicative of downslope re-sedimentation of unconsolidated sediment in response to earthquake generated underwater landslides. I suggest it was this type of message from nature that Alonzo Commanda was finely attuned to, in a disrupted but none-the-less profound thread of connection with traditional knowledge handed down from time beyond memory, constantly upgraded through intimate motional relationship with land and its language, even despite the massive and irrevocable transformations with the coming of the new people. I suggest here that by trying to understand how WC was teaching, I was gradually learning directly from the river itself.

Three: WC believed that it was critically important that the new people who now occupied his homeland develop a relationship with her. Then, he believed, they would be alerted to her voice and to the urgent environmental messages she had been shouting at him for decades. He believed the unique and special area and the ancient rock in the centre of the country at the Asinabka Sacred Chaudiere Site warranted urgent protection in terms of its critical hydrological and geological history and importance. Its embeddedness in a special landscape of spiritual significance strengthens this claim. The four years of constant challenge of colonial and neoliberal developmental practices at the site, as well as the 2007 public challenge of damming, participation in Circle of All Nations events on Victoria Island, and public engagement in other environmental challenges inspired by WC, suggest that many people are keenly interested to understand this Indigenous pathway to knowledge. The continued growth of his Circle of All Nations attests to the interest of growing numbers of unpracticed players. 
In antiquity, in understanding life on earth, most traditions cohered around the idea of an integrated great chain of being incorporating matter, body, mind, soul and spirit. With modernity, thinking shifted in the western world and cohered around dualities, binaries and segmentation, as well divergent views about religion (versus spiritual means of knowing, still vital in the Indigenous approach). These, amongst other things, impacted human relationship with earth, and today, environmental challenges and climate change urge a critical reconciliation with earth. This, I suggest, was WC's central epistemological project. WC's ancestors learned from the Laws of Nature. Knowledge emerged from relationship, respect and responsibility. He believed newcomers like me could learn this from the Indigenous teachers, their manner of storytelling and the Laws of Nature. He created a virtual school to animate such knowledge generation. To WC, in view of the accelerating global environmental crisis, it was critically important for all to develop relationship with a Mother Earth in keeping with the Laws of Nature.

My argument is that WC's ontology was informed by cartography and physical geography, his global outreach and ontogenesis was driven by human geography and environmental crises and that he animated an epistemology grounded in politics, humanities and Laws of Nature in his Circle of All Nations work.

\subsubsection{Language, Linguistics, Semantics, Semiotics and Performative Technologies}

A comprehensive grasp of the complexities of WC's discourse is critical for an analysis of any ongoing integration of his thoughts. WC's life spanned a century of significant manipulation and evolution of communications, inclusive of the near-annihilation of Indigenous language, the outlawing of custom, ceremony, song, dance, movement, and practice, and the introduction of new languages, radio, television, the camera and the computer. He developed his own repertoire to engage actively in contemporary times. The 
cybercartographic component of this research registers WC's anticipatory use of modern technology in his inter-active and bridge-building discourse, and this may account in part for the sense of him being encore vivant.

Language, linguistics, semantics, semiotics and performative technologies played a critical role in WC's discourse. This was especially evident as he grew older, when it was sometimes difficult to hear his actual voice. WC was born of Indigenous oral heritage. He grew up with the old Algonquin language on his tongue; the generic language emerged with the people of the Ottawa River Watershed. His use of language was precise. I note the following incident, to emphasize how critically important language and discourse was to him. Just before his death, he spoke disparagingly about a version of the Algonquin language reclaimed via the lens of the church after the early period of colonization. He said to me, "What do you think, the colonizers are teaching us our language!" (Thumbadoo Personal Journal 2011). His father and paternal grandfather did not associate with the church and were more distanced from its control and influence. Further they spoke an unadulterated Algonquin which held ancient knowledge. WC examined dictionaries critically (as he did bibles), and there were some that he did not approve of. Still, he did offer some approval of the Larousse Dictionary, which translated Algonquin into French. He understood and spoke most Algonquian dialects, all the way across to the land. He was able to speak to the Chumash people when he went to California in 1996. It is interesting to note that the language of the Chumash people share roots with the language of the Indigenous people of Baja California, Mexico. This attests to the reach of the Algonquian language family across the continent, and supports his assertion that there are eight four Algonquin nations that span the continent. This is the web of connection that informed the energy of Ginawaydaganuc and Mamininini. His second language as a child was French. Later, with 
exposure to American tourists, he strengthened his English. He shared two stories that reflect his facility with language (and his penchant for humour): "One day, this French boy called my dog a son of a bitch, and I pulled up my sleeves, and set to defend my dog. Then I thought, come to think of it, he's right!" This was followed by the WC chuckle. But there was nothing un-purposeful about WC's storytelling. In the seventies he went to France, and was invited to speak at a school; after he finished his speech in French, he asked the teacher if his presentation was all right. The teacher responded, "I don't know. We could not understand a word you said!" This time WC's retelling of the story and his chuckle were about both the discrepancies between the Quebecois French he had learned and Parisian French, as well as about the general colloquial Maniwaki/Quebecois French and Lac St Jean area class-conscious elitist French, and the associated rivalries and political conflict. On a more poignant note, I remember also that WC used language to manage pain. Though I did not understand much of his language, I knew its general rhythm, sounds and patterns. When he was in great pain close to his death, he reverted to an older version of Algonquian, to accommodate communication with other forces of life.

I discuss these stories to show why an understanding of WC's semantics is critical to an appreciation of the fineness and precision with which he linked his intentions with his thoughts and with his words. He repeatedly expressed deep pain and regret that he could not speak directly to others, and increasingly to his own people and family, in his own language. He had so much of importance to share and this could not be comprehended fully with thoughts and ideas compressed forcibly into the confines of the English and French languages; further, his language used verbs and not nouns; the name of a tree changed as it aged; sometime he was indifferent to gender words; and more often, he would use the feminine. It took me a while to truly understand the implications of these thoughts. WC was 
passionate about the protection and survival of Indigenous languages, seeing them as libraries of information, knowledge and wisdom that were dying with the passing of each elder. How could one be Algonquin if one could not comprehend through its words? he wondered. He was also cognizant of the irrevocable loss of larger ideas without the words to approach, encompass and interface with them. He saw, and linguists confirm, the relative simplicity of a language like English, when compared to the vastness of his language. With the loss of the words comes a loss of the ideas and conceptual thinking that gave rise to them. I myself studied English language and literature decades ago, and thought I knew words well. Today, I see researchers in human geography use words in new ways, to express new ideas. How much was WC trying to convey, to translate from the vaster stock of words in his Indigenous repertoire? I did not grasp all the implications of his words immediately. However, over the course of fourteen years of intensive dialogue, repetition, reflection, questioning and reframing, followed by the past six years of further review, I have learned to master an understanding of the nuances of the WC discourse, a discourse that was not just confined to a precise articulation of the complexities of the spoken and written language, but wherein other discursive elements were intuitively, consciously and meticulously constructed and performed.

WC used the word vast repeatedly to describe his language; I marveled at the geographical rootedness in this deliberately chosen word. I knew how precisely he used his words. He came to his choice via a brain map that took him from apprehension to Algonquin to French and then to English. The grasp of cosmic vastness was precisely what he intended to convey; but his audience lacked the capacity for comprehension, locked into narrower circuits. I noted this recently in rereading a mini-biography I had written about him for a historical publication. During an edit, unnoticed by me, two words had been edited: 
the vastword, and the descriptor of WC's canoe making craft tool, the crooked knife. Even here, where there was great interest and respect for the elder, there was also the constraining of Indigenous concepts to meet the needs and span of the non-Indigenous audience. The incapacity to stretch beyond reveals and underlines the challenges of the constituted Western discourse. There are limitations and implications to translations of French words to English too. For example, WC used the word pardon a great deal. It is a somewhat old-fashioned word, and it is frequently translated to mean forgiveness. I noted the following two explanations: forgiveness of a serious offence or offender; and (Obsolete): a papal indulgence. Further search on the latter provides this information: Roman Catholic Church: a partial remission of the temporal punishment, especially purgatorial atonement, that is still due for a sin or sins after absolution (www.Dictionary.com). It is interesting to note that the Algonquin word for purgatory is still used in the Algonquin language calendar in his community. One day in the week is the Day of Purgatory. WC had, in his archives, the graphic teaching scroll prepared by Father Lacombe for the easy education of the natives, and Algonquin today still discuss being terrified by it. No matter whether you have been good or bad, you have to pass through the fires of purgatory; and some burn there forever. The fire image of purgatory then takes on a devastating significance of its own. The Catholic understanding of hell fire was in direct contradiction to the original Indigenous attitude to fire. It was one of the four key life-giving elements of the natural world, and no doubt, to a peoples who evolved in a transforming ice world, one of crucial importance. An entire thesis could be written around the papal association of the word, pardon, given the forcible eradication of Indigenous belief systems that came with colonization, residential schools, and the near obliteration of language. WC's use of it conveyed particular messages to the French and Catholic peoples in his audiences that were perhaps lost on others. I note that his daughter, Evelyn Commanda Dewache 
frequently describes a difference in the tone of elderly French/Catholic folk compared to the English. She believes that they are more fearful and distraught as they approach their graves, being more deeply indoctrinated in Catholic ideology.

I also suggest that beyond word meanings, we need to try to comprehend the awareness that we have no words for in English or French, but that WC nonetheless appears to have awakened in diverse peoples. Further, there are also European words that the Indigenous had no translations for. We are very interested in a few key English words that are not present in WC's original language. WC's friend, Algonquin writer and researcher, Evan Pritchard, wrote a book, entitled No Word for Time: The Way of the Algonquin People (Pritchard 1997, 2012 Millichap Books). I also learned that there was no word for map in Algonquin. Now, these two concepts and are fundamental to geography, and fundamental to the lives of WC's peoples; by what means beyond the literal translations did they express these, then?

WC's daughter described a lawyer who visited her father as a liar; and in my own work in justice, I learned that the Inuit understood a lawyer as the one who would lie for you. Another important one is the word Justice: I recall Mi'q Maq elder Alec Denney showing me how one word of his language conveyed a page full of meaning and information. Further, he added, "in our languages, we have no word for justice; if someone does something wrong, then it means, either they have not been taught well, or they are sick: our traditional structures were focused on teaching our children, and ensuring health and balance" (Denny Personal Communication 1996). In my twenty-five years of work in the Canadian criminal justice system, I have noted the same with wrongdoing. Bert Hellinger, a former Catholic priest who had spent sixteen years working amongst the Zulu people in South Africa and creator of a powerful new systemic constellations therapeutic approach to address multi-generational 
stress and physical and emotional ill-health, (www.hellingerinstitute.com) remarked to me, Just imagine the implications of a society that has no word for justice! WC conducted a pipe ceremony at the first ground-breaking systemic constellation conference in Germany in 2001.

Pattern and rhythm are employed in complex ways in communications, and sound introduces new dimensions to words. WC's use of words emerges from the oral, and the physicality of speech and connection with breath and oxygen reveal themselves as inherent parts of communication; the critical importance of breath, sound, words and communications to the survival of peoples living in cold regions of the world is still alluded to and animated in the throat singing of the Inuit.

A close examination of WC's public practice, performance mapped at multiple levels, over the course of several decades, and more intensively over the past forty, reveals power generation via interaction with the unseen force. His chosen word for this unknown force is Creator. Here he asserts connection with the procreative, evolving, emergent, and multidirectional, temporal dynamic.

Song, interwoven with dance, constitutes the critical components of communications. Both were employed in the first encounter of Samuel de Champlain with WC's ancestral leader, Tessaout, in Tadoussac in 1603. Beyond the scope of this study lies the significance of sub sonar messaging of drum beat. Sound and linkage with the larger elemental world were incorporated and materialized in naming words. WC's great, great grandfather was Pakinawatik, Stick Hit by Lightning/Thunderstick. His oratory skills were legendary, and his power was compounded by the connection with the energizing, electrifying light and sound created in the skies. The same applies to WC's own Indigenous name, Ojigkwanong, Morning Star, inclusive of cosmic space/time knowledge and dark/light sensory referents.

Language was one means of communication in WC's discourse; but he employed 
many more. The actants in his discursive repertoire that typically accompanied all his public communications reveal an agency of force beyond the acknowledged material world. Generally drum song, reflective of the heartbeat of Mother Earth, alerted visible and invisible beings to the activity on hand, and drew the attention of all to the focal point: the doorway into the moment, place and experience. For WC, after the energetic awakening of the drum, the formal ceremony commenced with a smudging ceremony. Here, one of the four sacred herbs, for him generally sage, was burned in a shell, and his feather used to fan the smoke around a person. This gesture of cleansing, protecting, balancing, healing and focusing was accompanied by words in Algonquin. For podium type meetings, he smudged a group from afar. But he generally preferred to have people assembled in a circle, and he liked to have them turn around in a full circle, following the direction of the Sun (east to south to west to north: the heliocentric directional spatial measure). He thereby encompassed them in a circle of groundedness that also emanated temporal, cosmic and intangible connection in the smoke fanned into space by the mobility and temporality of the feather. As individuals drew the smoke to their eyes, ears, mouths and limbs, they were reminded to focus their senses on the matter at hand; thus, intangible, physical, mental and cosmic lines of communication were threaded together in this initial energetic threshold into the communications. This resonated for individuals and groups, irrespective of background, attesting to a reach to some common denominator in the psyche; I have witnessed the impact of WC's smudging ceremony thousands of times with multiple audiences. In addition, there was dress, sometimes traditional and iconic, sometimes not, but always, distinctively WC's. In particular I note his use of his headbands, perhaps implying an authority from above or beyond, and rings, perhaps denoting energetic inter-connections.

When WC spoke formally, he first brushed his lips with his feather, to ensure 
support that only the best words would be spoken and he actually addressed his Creator first. His communications began with his Creator. This Creator was not the Indigenous version of God for him. Rather, Creator appeared to approximate the creative principle of life, and more beyond that. Consistent with his belief that his language evolved with life itself, he frequently noted that it contained no cursing words. He addressed his Creator in his Algonquin language, and then translated himself into English and French.

One may ponder what role the feather played in his discourse, as he commenced his speech by brushing a feather across his lips. It has now become common knowledge that Indigenous Peoples consider the eagle a sacred bird, a direct link with Creator. An eagle feather, gifted to a person, found or acquired, is a symbolic statement that its bearer is a person of power, a person with direct link to Creator, or perhaps needs healing or guidance. WC generally used a Condor feather in public events. What was he asserting? Well, he said it directly many times: "I did not create the Panama Canal". Neither did he create the division of Canada, the United States and Mexico, nor the separation of Quebec and Ontario. Instead, he performance mapped the geographical integrity of the continent by emerging everywhere himself. By affirming this relationality from the personal, he dismissed objectification. But he also moved beyond subjectivity, instead invoking an Indigenous collective. In his use of the feather of the Eagle of the South, he asserted seamless interconnection across the Americas, animated within the context of a semiotic landscape also populated by Indigenous prophecy spanning space and time, in pentimento fashion. This was a reference to the prophecy that foretold the coming together of the Eagle and the Condor, the reunification of the people of North and South America. This manifested when Indigenous leaders of the south presented him with the Condor feather in the nineties. These and other referential points create the unique iconography and deliberated messaging 
of the WC discourse. The fact that certain elders now seek to assert the same attests to the discursive power of his technology. Effective execution, however, depends on the critical interweaving of authentic visible and invisible energetic elements - and therein lies the reach of the virtuoso shaman. WC said,

In his opening remarks at the UNESCO Millennium Conference on Art and Culture,

I am in the earth, wind and waters;

I am as the bird flies; and the wind blows and the water flows.

(Thumbadoo 2005 35)

With his use of his feather, brushed to his lips, he asserted truth and earth emergent authority in his own iconic way, and in a time-space moment of global inter-connection. More than a decade later, National Archives librarian Gwynneth Evans informed me of his impact (Evans Personal Conversation 2012).

While WC was a vibrant and prolific communicator, he became increasingly conscious of ensuring that he reached his diverse audiences with clear messages that they could ponder further. When we became acquainted, following his observation of my work hosting a series of Aboriginal Justice conferences, and the development of our relationship, he wanted formal speeches to be incorporated into his addresses. As he got older, these written speeches had to become increasingly succinct, and eventually only occupy one page of large text - perhaps he was preparing us for Facebook! A comparison of his written speech, and the spontaneous convocation address he delivered when he received his honorary doctorate degree from the University of Ottawa, confirms how aligned the ideas were. I note also a poignant moment, towards the end of his life. At this time, he was growing increasingly incapacitated by kidney disease and dialysis treatment, when treatment sometimes impacted the clarity of his thought and delivery. He was involved in the opening for the revitalized Museum of Nature, and the bright sun prevented him from reading his 
notes. Nature was a passionate concern for him, so he had a lot he wanted to share, and he did. But this was an outdoors launch with the public at large on the street in the hot sun. Later he worried about not delivering all his key points effectively, despite the smudge and feather, prayer and oral words. This in part explains WC's intense focus on his audience. It also helps me justify this admixture of academic and oral storytelling to bridge across vastly different knowledge bases and starting places in exploring his discourse.

WC's understanding of land emerged from his language. Language was critically important to him. Countless people have affirmed how seamlessly Algonquin, French and English languages were used by him and his wife in their daily communications. Algonquin is a land-based language, wherein, according to $\mathrm{WC}$ in his analysis and articulation, the land itself, its sounds, its physical, geological and biological realities (rocks, stone, animal, plant and the elements) shaped and determined the evolution of the language. The generic language which emerged with the Algonquins of the Ottawa River Watershed was transmitted across the country by his nomadic ancestors, and he emphasized that there were 84 Algonquin Nations across North American that spoke dialects of the mother tongue. His Algonquin friend historian, researcher and writer, Evan Pritchard (Native New Yorkers, 2007, Henry Hudson and the Algonquins of New York, 2009) drew him a map of North America identifying these tribes. WC was raised on the ancient Algonquin. While confined to French and English in his communications with others, WC nonetheless performed his own and much vaster understandings of truth and meaning. The import of his words and other dimensions of communications warrant careful examination to grasp an understanding of the depth of his thoughts and by extension, this applies to the understanding of Indigenous peoples and their communication styles.

An exploration of semiotics, textuality and choric expression contributes to an even 
deeper understanding of the Commanda discourse. Semiotics (from the Greek semeio, to mark) is also a study of meaning-making, here privileging signs, sign process and nonlinguistic sign systems as a significant part of communications.

A few key items in the Algonquin semiotic repertoire include petroglyphs, pictographs, feather, drum, tobacco, wampum and canoe, the latter both a pragmatic and iconic motif. These objects served as metaphoric devices, and the wampum belts, his condor feather, rocks and other iconic and bio-semiotic signs of intelligent nature informed WC's thinking and played foundational roles in his discourse, as did reference to animals. Further, I note in our handwritten notes that these quotations from some unknown source preoccupied us 'Train your eye to see, and you will cease to read the world'; and 'readers and see-ers can't relate to each other'. It is interesting that this see-ers word is similar to seers. I came to my knowledge by reading first; and WC by seeing first; then we had to work at aligning our articulation of what resonated as integrative knowledge; in our joint engagement with the public, he would often ask me to explain certain things and initially he would make a gesture of winding me up behind my back, my oral style being hesitant; this entertained his audience. Later, as I acquired greater fluidity in his style of communications, he would sit back and smile.

Another entity inherent to the multi-directional style of WC's discourse is textuality/inter-textuality. Textuality is inclusive of written and spoken words, maps, photographs, sketches, videos, and, I add, song and dance, talking circles and gatherings, divisible into genres (categories organized by style, form or subject matter); and intertextuality, wherein meaning is produced in relationships between texts (Waite 2010). His repertoire was rich and diverse and multiply constituted by his vast Circle of All Nations. 
Casti notes that with the re-reading of conquest maps, cartography took on the unprecedented role of establishing the arena for a geographical decolonization leading to counter mapping challenging hegemony and asymmetries of power by colonized or subjugated voices. This critical cartography requires reflexivity. Reflexivity offers a set of tools not only to understand the past, but to rethink what we do, and provide suggestions for future improvements (Casti, 2016). Casti proposes a heuristic approach, "capable of holding together the outcomes of cartographic and of geographical theories, the artistic hybridizations envisaged by historical cartography, and the possibilities offered by digital technology ...(inclusive of a) semiotic perspective ... (of) the communicative potential of maps" (Casti, 2016 xiv); she terms this chorographic cartography, and it includes the experiential.

These many concepts (and more) are inherent to and complement discourse analysis. They contribute to the understanding of ontological and ontogenetic components of cognitive and material communications and mapping. Indigenous realities are densely communicative at these levels, and it is of critical importance that they are acknowledged and interpreted as accurately and honestly as possible, while their emergent and shape-shifting integrity, inclusive of depth recall, reflexivity, iterative, and immediacy, collapsing past/present, is also recognized and legitimized as knowledge generative. Indigenous respect for this has revitalized the use of the term elder. Knowledge derived from experience is deemed wisdom in much Indigenous thinking, and is highly valued.

\subsubsection{Algonquin Ideology and Knowledge Generating Practices as Embedded in Rock Art and Animated in Wampum Agency}

In the Foreword to the publication, Sacred Art of the Algonquin A Study of the Peterborough Petroglyphs (Vastokas, J.M. and R.K., 1973), Waubageshig, Native Studies Program, University of Trent, wrote: 
"Too often in the past, publications which dealt with native subjects tended to represent the academic preoccupation of their authors which had the effect of obscuring their usefulness to the casual reader, while ignoring the aesthetic and creative facets of native cultures... This study of the Peterborough Petroglyphs is a prime example of the kind of work which is invaluable not only for its scholarly treatment of a part of our native heritage but also for the information and analysis which it provides about an aspect of traditional native culture which, for the most part, has remained a mystery to Europeans since their original 'discovery' by them. The fact that this study is not exclusively academic in scope should make it very acceptable to native researchers as well as all students of Indian culture" (Vastokas 1973 vii-viii).

This introduction suggests the bridge building potential of a study of the most ancient evidence of the art and spiritual thinking of the original peoples of the American North East. The Vastokas team conducted significant research into the Peterborough Petroglyph site during the summer of 1967, fifty years ago, and published their book as a report on the recording and excavation of the site. It also attempted a cultural placement and interpretation, addressing descriptive and interpretative interests in this exceptional monument of prehistoric Canadian art. There are over three hundred identifiable and hundreds more obscure glyphs incised on a single outcropping of crystalline limestone over a considerable period. Many of the petroglyphs bear similarities to images found in other pictograph and petroglyph sites in the Canadian Shield and elsewhere, and they note that "works of art and the artistic process are, in fact, central to the interpretation and understanding of a given way of life" (Vastokas 1973 2). They add that iconographic analysis seeks to identify, describe, and classify pictorial images, subject matter, or recurrent motifs with reference to the tales, myths, or other verbal and literary products of the culture or period during which the work of art is made, with the art, like language, having an inner logic of its own, inclusive of not just the individual elements of the art, but also of their relationship to each other, format, ground and surrounding spatial environment. These serve much like the underlying rules of grammatical structures in language. 
WC had a copy of their book, marked x3, (perhaps they presented him with this copy) from the time of its publication in 1973. Their findings resonated with his understanding of his cultural heritage gleaned from language, canoe making, life 'in the bush', the knowledge he absorbed from his family and from being around older people, and his own research into the history of Indigenous Peoples of the America, from his earliest days. He himself had visited the site in 1952, and he related to me that the waters now cemented off, flowed freely then. In 1954, the site came to the attention of the province and interest led to its transformation from its former obscured state, and to its preservation as an educational and historic site of prime importance. This attention resulted in an awakening to the art of the prehistorical Algonkian peoples, Algonkian being the term used to include all people using the Algonquian language and not just the Algonquins of the Ottawa River Watershed, themselves referred to as Algonkins in this book. It notes that the site lies en route to Georgian Bay, Manitoulin Island and the Great Lakes, all part of the original territory of WC's ancestors. Note that while the book deems the petroglyph research most relevant for the Algonkins of the Ottawa River drainage, and the Ojibwa of the northern shores of Georgian Bay and Lake Superior, our other research informs that Manitoulin Island was the original home of the Algonquins of the Ottawa River Watershed. They travelled the entire route of the mother river, the St. Lawrence, birthed in Lake Superior, travelling through the Great Lakes and then entering the Atlantic Ocean in the Gulf of the St. Lawrence. The Vastokas team notes that the Algonkians living in Ontario and around Lake Superior lived a more or less common way of life, hunting, fishing, and gathering wild foods; they comprised small independent, and amorphous clustering of one to four hundred people, associated with geographical territories that they accessed in the autumn and winter as even smaller family units, subsisting on beaver, moose, caribou, deer, partridge and fish; 
with the hunter, in particular, leaving the family's winter dwelling to return only after a successful hunt. After the winter, they would leave their hunting grounds to reunite as a larger unit near a lake, stream or river where they spent the summer to fall fishing, hunting small game, gathering medicines, berries and wild rice, following the time/space order of nature. This pattern of Mamiwinini movement/seasonal rounds was inherent to the intrinsic motional nature of the people that WC alluded to repeatedly, that was crushed for most part by the reserve system, but that he managed to assert by constant modern day travel, local, regional, national, continental and international, throughout his long life. The Vastokas team notes also that the birch bark canoe was probably the single most important item of Algonkian material culture, rendering accessible the vast network of lakes, rivers and streams in the Great Lakes region. Further, it served as a medium for art (Vastokas, 197330 - 31); this will be examined further in the context of WC's canoe making heritage, epistemological legacy, and emergence, in Chapter Six.

In their efforts to examine the rich pictorial character of the site which allows a glimpse into the non-material, spiritual, and intellectual life of prehistoric Indian culture (there is much relevant discussion on pages (Vastokas, 197332 - 35), the Vastokas team examined the perspective of several scholars, including Paul Radin, (an authority on Algonkian ideology and world view, whose pioneering study was entitled Primitive Man as Philosopher); affirming his findings, they note:

"so called 'primitive people' were profoundly practical, empirically-minded, and realistic in their everyday relationships with nature and in their knowledge of the external world. The 'thinkers' or intellectuals among these hunting societies, moreover, interpreted the empirical world around them in metaphysical terms, systematizing their reflections into sets of cognitive principles, which were often embodied in oral tradition and in religious ritual. As a result of his investigations, Radin... concluded in 1927 that the customary view of the intellectual history of mankind had to be completely revised. The Vastokas team go on to point that this was a conclusion re-emphasised more recently by Irving Hallowell: Human beings in whatever culture are provided with cognitive orientation in a cosmos; there is "order" 
and "reason" rather than chaos. There are basic premises and principles implied, even if these do not happen to be consciously formulated and articulated by the people themselves... If we pursue the problem deeply enough we soon come face to face with a relatively unexplored territory - ethno-metaphysics. They also examine the research of Claude Levi-Strauss, who had noted the extreme familiarity of hunting peoples with their everyday, biological, and physical environment, so much so that the most minute differences among species of flora and fauna are noted, named, and classified; they also acknowledge his conclusion that this knowledge is not limited solely to the practical, economic ends of survival, but that it serves to meet intellectual and philosophical requirements... and that an essential need of man the world over is his desire for system and order and the classification of observed phenomena and details of the environment as a means of introducing order and coherence into the universe. Clifford Geertz, they note further, in his discussion of religion, sees as universal the search for intellectual lucidity, since the strange, the uncanny, and the elusive must be accounted for and placed within a people's known body of knowledge. Moreover, religion allows the projection of these "images of cosmic order" into the everyday, comprehensible plane of human experience, and without this ordering, Geertz tells us, man "would be functionally incomplete ... a kind of formless monster with neither sense of direction nor power of self-control, a chaos of spasmodic impulses and vague emotions" (Vastokas 1973 33).

While I question the latter view, I accept the key point: that religion or like sentiment,

like art and science, functions in part to establish order, to create a meaningful, systematic whole out of sensate experience. I argue though that in WC's narrative, this order is derived from the Laws of Nature.

Based on their research, the Vastokas team identifies the following as critical constitutional aspects of Algonkian world view: the concept of manitou, guardian spirits, vision quest, shamanism and cosmology.

They suggest that to the Algonkians,

"the entire universe was charged with meaning, with power, with manitou. Manitou was the vital force, spirit, or energy in all things, both natural and supernatural. It formed the essence of trees, plants, the sun, bodies of water, stones, and such forces of nature as thunder, lightning, wind, and rain. This world view, however, made little distinction or separation between 'natural" and 'spiritual", nature itself being sacred, and the world being filled with spiritual meaning" (Vastokas 1973 33).

This natural/spiritual link aligns with the WC understanding of things. He also made a huge distinction between spirituality and religion, seeing to a large extent religion as a man 
made tradition of domination, in war against the spirit of nature, operational and destructive in the lives of both his ancestors and Mother Earth, from the time of the coming of the first colonizers.

The Vastokas team go on to note that Algonkian shamans were differentiated into three specialized groups - Jessakkids, Wabanos, and Medas. I note that in the book, The White Chief, written by Bertha Wright Carr-Harris, and affirmed a true report of her father's notes, she makes mention of the Algonquins from Petit Nations (WC's direct ancestors). They challenged Philomen Wright for cutting their sugar bushes at the Chaudiere Falls; then their Jessakkid shamans arrived to conduct a peace-making ceremony (The White Chief of the Ottawa by Bertha Wright Carr-Harris, reporting on 1806-1816 notes of her father Philomen Wright in William Commanda's Personal Archives).

It is chiefly an understanding of Jessakkids and shaman that preoccupy us now. Later in the thesis, I turn to the conceptualization of guardian spirits, vision quests and cosmology to query their place in WC's ideological framework, and his discourse. I add that much as the priests renamed Indigenous spiritual sites as sites of the devil, so to they attacked and rendered negative the meaning of Jessakid, such that in contemporary times, it is often used as a cursing word. They were most resistant to the influence or will of the priests, much like the tribe named Tetes de Boules, or bull-headed by the priests (William Commanda, Personal Conversation).

The Vastokas team, drawing on the research of Hoffmann from 1891, notes that the Jessakkids were specialist prophets, seers, and diviners. They could see into the future and predict outcomes. They generally formed no group and they practiced alone; blessed by the sun, they were assisted directly by Turtle and Thunderbird manitou, of which they would have dreamed in their youth. They conducted Shaking Tent ceremonies, within a ceremonially 
created cylindrical, roofless tent, from within which, as it shook, they would be able to access and interpret messages from beyond about the future.

WC was believed by many to be the carrier of the Seven Fires Prophecy wampum belt at the time of the unfolding of the Seventh Fire; the following is an excerpt from Learning from a

\section{Kindergarten Dropout:}

This morning the Elder wanted to focus on the message of the Seven Fires Prophecy. This is a prophecy that has been carried as a fundamental part of the oral history of his direct ancestors for at least six hundred years; it is also a well-known prophecy amongst the Ojibwe people and many other tribes across Turtle Island. But in fact, we have been told that the prophecy is even more broadly dispersed, including amongst indigenous peoples in Africa. This was brought to our attention most dramatically by Zulu shaman, medicine man and writer, Credo Mutwa, when we visited him in his home in South Africa (just after the 9/11 attack; for WC, this moment was a significant marker in the prophecy). As William started to relate the story of the sacred wampum belt, he said, you hardly need to tell me, I know it already; it is in the legends of my people too.

In essence, the prophecy tells of the future of the Indigenous peoples as it was to evolve over the years following the coming of the new peoples to their land. The prophecy was brought to the people by seven prophets, two of whom appeared together as one. These prophets described the major transformations that were to come into the lives of the people over different epochs of time.

Thus, the first prophet told of the time when the people were living a safe and secure life, the second warned of a danger (new people) that was coming to their lands; the third told how those who had not heeded the warning and taken precautions to move away from this danger would be destroyed; the fourth prophecy was delivered by two prophets, and theirs was the message of duality: they warned that they could also be enemies under the disguise of friendship - again, the people were warned to be on guard. The fifth prophet warned of the false promise that could come under the guise of religion. The sixth prophet told of the pain and turmoil that would devastate the lives of the indigenous peoples who had turned away from their old values and ways of life with the coming of the newcomers. The seventh prophet talked about a time of choice making for all - for continued exploitation of land and peoples, or for a renewed respect for Mother Earth and reconciliation between Indigenous peoples and the newcomers. The double diamond at the centre of this eight diamond belt reflects this hope for unity to emerge out of the duality.

It is a rich, epic prophecy that appears to emphasize the importance of choice (and consequences) throughout - individual and communal choice - and this choice appears to be no more important than at this time.

As noted, William is seen by many to be the carrier of this sacred wampum belt at the 
time of the unfolding of the seventh prophecy. Will we come together to light the eight fire of peace and reconciliation with each other and Mother Earth? He states that on September 11, 2001, the light skinned race were given a choice between the roads to peace and reconciliation or destruction; he notes daily that the path taken by many has plunged the world into ever increasing war and bloodshed - and the balance of life has been irrevocably shifted.

But even as he sees such destructive choices as have been made to date, he still holds out hope for a Circle of All Nations, A Culture of Peace and continues his tireless work to this end, as is his responsibility as the prophecy carrier. For he believes that there is still time for the other face of the prophecy to ignite.

Once I asked William how the story could have been preserved over the centuries well, he reminded me, five or six hundred years is not so long; people used to live a hundred years or more, and people four or five generations apart could have known each other, thus it was possible to retain the intimate connection with messages stories, histories and legends; though made more difficult after the deliberate efforts made to destroy these records of history, and their languages of transmission, the powerful spiritual messages remain strong and compelling. William himself and his older sister Judy both knew their great grandfather, Pete Tenesco, who assumed the care taking of the Wampum Belts after the legendary Pakinawatik died. (Note, their grandmother, Manianne Tenesco Commanda, grand daughter Pakinawatik, was the first daughter of Pete Tenesco through his first marriage to Philomen, daughter of Pakinawatik; WC himself heard stories about the belts directly from her and his father, Alonzo Commanda.)

In the mid-nineteen nineties, William met Eddie Benton Banai, the Ojibwe Elder from Bad River, Minnesota, at a conference in Peterborough, Ontario. The Ojibwe Elder was making a presentation on the Seven Fires Prophecy, and his graphic presentation reflected the key ideas associated with the sacred wampum belt William carries. After the presentation, William showed him the Belt. Is this the Belt you are talking about? he asked. The elder was greatly moved and told how the story of the seven prophets had come to him in a vision. He wrote this vision down. William often shares this written version of the prophecy when he shows the sacred wampum belt, since it captures the oral history so graphically.

But, of course, since it is an oral heritage that the prophecy emerges from, and since it is a live entity, it continues to speak its messages in a vibrant way to its carrier. Thus WC states that 11 September 2001 was the pivotal marker in the present history of the Belt and this lends tremendous urgency to his work (Thumbadoo 2005 51,52).

Alerted by the Laws of Nature, articulated in wampum prophecy (what Zulu shaman, healer, writer artist and mapper, Credo Mutwa, described quite simply and brilliantly as early warning signals), WC presented his counter discourse in pentimento fashion. He exposed 
multiple other ways of knowing, and shone the torch on many festering layers in the time/space history of his land - not, as he said, with boxing gloves to annihilate entropically, but determined to challenge, stir and animate negentropically.

\subsubsection{Spirit Energy of the Land}

The performance mapping logic of inquiry contextualizes this examination of WC's web of knowledge. A challenge for my research project was to determine what such a loaded word as sacred might mean in the context of the WC discourse within the domain of geography.

Sacred: Collins Westminister (1960) dictionary offers: holy; pertaining to worship of God; consecrated to God; divine; religious, as writing, history, etc. dedicated. The New Webster Encyclopedic Dictionary offers: to set apart, to consecrate; Fr. Sacrer, from Latin sacare, from sacer, sacred (seen also in sacrilege, sacrifice, consecrate, desecrate, etc.); same root as in sanction, sanctify) set apart by solemn religious ceremony; dedicated to religious use; holy; not profane or common; relating to religion or the services of religion; not secular; consecrated; dedicated; devoted: with to (sacred to one's memory); entitled to the highest respect or reverence; venerable; not to be profaned or violated; inviolable; inviolate (a secret kept sacred) (Collins Westminister 1960; The New Webster Encyclopedic Dictionary of the English Language Including A Dictionary of Synonyms and Twelve Supplementary Reference Sections, Processing and Books, Inc. (1952 - 1971) Winnipeg, Manitoba).

Geography is concerned with space and time, and I address the where and when questions together. From what I have learned from and about WC, and his distinctly Indigenous approach, space and time are inextricably woven together in cosmic space, and all matter, seen and unseen, is relationally linked in multiple and diverse ways. For him the roots of the human search for life are grounded in earth, and he believed the thread of associated knowledge came down to him from an ancient source: the Paleo Indians who inhabited the American North East from the time of the retreat of the Laurentide Ice Sheet around 12,000 years ago, and who learned to coexist with the Laws of Nature and wrestled knowledge directly from their world. While there are many academic understandings of the term, Laws of Nature, I note that WC used it frequently to express the complex mix of 
conceptual, physical, experiential and intangible knowledge.

WC's Law of Nature ideological framework emerged from land, language and associated ways of knowing, constituted in part by journey, fluidity, wampum agency and multiple inter-active practices. I probe its relevance for contemporary environmental knowledge acquisition, generating and processing. At the same time, not all that can be known can be expressed in words. There were many ceremonial and spiritual practices that were part of WC's approach to life. For example, tobacco, pipe and sweat lodge ceremonies were part of his life and lifework, he shared these with others, and they impacted many people and their evolving relationship with Earth, irrespective of whether they were Indigenous or not. These are only noted if particularly appropriate, to contribute to the understanding of the contextual and embodied discourse, and the integrated spiritual dimension of his Medicine Wheel approach.

WC's family are the earliest known inhabitants of Lac Commandant, now known as Lac Papineau. This Indian Island (which I visited) was the place of his maternal ancestor, Pinokwe Commandant, and she would have been living there in 1760 (the times of Pontiac's uprising and the subsequent creation of the Royal Proclamation of 1763). I recently showed WC's eighty two year old niece, Mary Daisy Commanda Jacko, a map of the lake. She immediately saw a body in motion in the image: Mamiwinini running. Others, non-native, have also seen a spirit man in the outline. The nearby Lac Simon was also a place inhabited by WC's ancestors. In fact, says Ms. Jacko, that lake would have been identified as Lac Chimaan to her ancestors, and the word chiman means canoe: a canoe-building Commanda ancestor is associated with this area. On that lake there is an island, and on the island, there is a lake called Lac des Etoiles. As told by Jean Fournier, and recorded by genealogist and historian, Jean Guy Paquin, (Le pays de Canard Blanc 2004, Au pays des Weskarinis 2014) 
this was a sacred place to the Algonquins; from here, they could see a mirror reflection of the sky and star world. What they observed was the prolific star creation factory of the Milky Way. They were below what we today know (via advanced technology) to be one of the two spiraling arms of our galaxy. This place was a tremendous source of cosmic knowledge acquisition. To this day, the August Persoid meteor showers are acknowledged in ceremony by the Narrangaset Algonquian peoples of the American North East (Harris, D. et al Indigenous American Ceremonial Stone Landscape of the Northeast 2017 Final Draft - in Circle of All Nations archives). August was an important cosmic time marker and WC always hosted his annual Circle of All Nations gatherings in August. WC's father Alonzo Commanda was one of the last people whose path to knowledge was directly via the stars and fire, the land and waters.

Sun was the critical cosmic body of fire that brought life from the east to an earth reclaimed from a retreating ice world, and that enabled the ancient peoples of the American North East to travel and live on the transforming land. Korp's research into the orientation of the Serpent Mounds in Ohio determined that they were not aligned with compass readings, as had been assumed by scientists, but rather with the rising sun (Korp, PHD Earthworks: Shamanism in the Religious Experiences of Contemporary Artists in North American 1991; personal conversation August 2017). The association of the words waban, wampum and wampeoag with the People of the American North East, the Algonquin, links dawn, light and wampum ideology. The significance of key natural features of the cosmic world is revealed in the time/space geo-cosmic knowledge of the peoples of the American North East. What they learned from intense observation of the natural and stellar world was reflected in their survival during transitional times in earth's evolution and in the ideology they developed. This ideology was registered in part in rock art: petroglyphs and pictographs, 
and also in language.

Cosmic knowledge featured dramatically in WC's mind. He told me many times about an old man from Obedjiwan, Quebec who came to join a gathering of elders camped out on Bitobi Lake. This was in 1922, and WC was a little boy of nine at the time. Unobtrusively, he served as water boy, and so learned from the old people in the traditional way: observing, listening, sensing, quietly paying attention to words and energetic nuances. This old man, whose presence was etched powerfully in his mind, talked about the features of the moon, the grey rock, the craters, the granular surface and its other qualities. When man walked on the moon in 1967, WC said he noted that the description of the moon was exactly as the old man had revealed it. When the people woke up the next morning, the old man's tipi was gone; but his memory and message remained encore vivant to WC. The importance of the Moon then was deeply impressed upon him. It is interesting that an Indigenous prophecy of Indigenous elder Peter Ochiese foretold the Landing of the Eagle; Angela Roulette linked that with the 1967 walk on the Moon (Personal Conversation September 2017). 1967 was also the year of WC's first major cross-border gathering and formal assertion of Indigenous presence in North America. Native American lore is alive with anecdotes of heroes like Geronimo and Sitting Bull being like wind on the plains during the Indian Wars. His own uncle Gabriel Commanda carried the famous Grey Owl, who had shot himself in the foot, safely through the battlefield in World War One.

WC was at the Sandton Centre in Pretoria South Africa in 2001, following the failed United Nations Conference on Zenophobia and Racism. Suddenly, the leaves blew around in a formation at the outdoor café, and he said to his companion, something bad has happened to the world. Later, we learned about the $9 / 11$ attack on the Twin Towers in New York. I learned also that a blood relative of his had had a similar experience with leaves, 
this one in North America, and at the same time. Had I not heard this latter story, I might have thought WC's environmental scanning practice was functioning very efficiently. After all, he had just participated in the racism conference, witnessed protests, noted the mounting tensions, watched the news, and determined the likely implications for global politics and escalating tension and violence. But, beyond this, there were other signs he was alert to, and other sources contributed to his awareness and knowledge.

Five other people and I witnessed an intriguing moment at a retreat centre that had been a former concentration camp in South Africa. WC had been asked to do a prayer, and thereafter, there was an audible response. He interpreted that as acknowledgement of energetic healing. I recall an unusual experience when WC was at the Shivananda Yoga Retreat Centre on Paradise Island in the Bahamas in 2001. The then eighty eight year old was very restless and could not sleep. He did a prayer for energetic healing and to regenerate relationship with the land. Later we learned about the first massacre of Indigenous Peoples (Taino and Carib) in the New World more than 500 years earlier. The 1995-96 Sunbow Five Walk across America brought his prayer to land violated and desecrated by warfare and violence (McFadden Odyssey of the $8^{\text {th }}$ Fire http://www.8thfire.net). His father had told him about the special power of an old native man. A mighty tornado was looming, and heading for his home. The old man readied his sacred pipe, and at the critical moment, he lit it and blew his smoke at it. The tornado moved away from his home leaving everything intact. From the time of his arrival in North America, Albert Einstein was in close contact with the Hopi; in fact they made him honorary chief. Pentagon officials consulted Indigenous peoples regularly. WC himself met officials twice. Pauline Joli de Lotbiniere, a researcher in Anthropology and Sociology interviewed WC for her article, Of Wampum and Little People: Historical Narratives regarding the Algonquin Wampum Record (in The Algonquins 
Clement editor 1996). She explores and legitimizes a type of energetic or morphological field of knowledge for the academy in some depth. While it is challenging to justify how stories like these that border on the esoteric configure in geography, they reveal the accessing of multiple other ways of knowing and of relationality, some only now becoming available through technology to modern science.

As a startling example of the frequent prescience of his awareness and its link with environmental challenges, I recall his meeting with the Chairman of the National Capital Commission, Marcel Beaudry, in 2004. Seeing limitation in the bureaucratic discussions about corporate law and constitutional law concerning the Chaudiere Site, which lay at the heart of his traditional territory in Ottawa, he warned of the potentially devastating force of the Law of Nature in reaction to being constantly impeded and thwarted by human actions. The very next day, the power went out across the eastern seaboard; 14 years later, it is still registered as the costliest environmental disaster to date in the United States. What signals was he noting to be able to speak with such certainty about that impending environmental catastrophe?

This spring, the spring of 2017, the Ottawa River itself became an active relational agent in the evolving Commanda discourse, here with reference to the heated Asinabka Sacred Chaudiere Falls land challenge currently underway around which the term sacred spins, in unprecedented flooding and environmental damage; WC's warnings startle again, as they did at the time of the 2004 meeting with the National Capital Commission.

In 2010, and at multiple other moments, WC articulated his understanding of Spirit.

As I have said before, inherent in the prayer of the Indigenous Nations of Turtle Island is the deep knowledge that we are all connected - my people in the east say Ginawaydaganuc. The prayer is a celebration of the profound knowledge that we are connected with the each other, as well as with the chief elements - Mother Earth, Water, Air and Fire - the animate and inanimate, the plants and animals and the larger universe, connected energetically. 
Spirit embraces and unifies us all.

Inherent in the prayer is a deep respect for both Mother Earth, the penultimate provider and nurturer, and all her children. The prayer is a constant reminder to honor this connectedness, and walk gently in the places of differences, for those are the places of co-creation.

But across the globe, our relationship with Mother Earth and each other has been disrupted, and storms and blood despoil our world. When the new peoples arrived in this continent, they were too often motivated by greed, the realities of the day were war and genocide, and the ramifications were environmental exploitation and destruction; my ancestors were nearly all destroyed. The land carries a deep pain.

We are now all feeling the impact of the environmental crisis that has resulted from the reckless exploitation of land, water and air, in escalating climate change; lands and peoples across the world are embroiled in desperate wars; and health is a primary concern across the world, no matter how privileged we might seem to be, for life itself is under attack.

Today, the Law of Nature is revealing herself as the great equalizer on all planes, with ominous implications for us all and for future generations.

Again, may I say our ancestors always knew that all things were connected, and our ancient prophecies told them that one day, all the world would one day come to us to learn this. As I mentioned earlier, I have been the Carrier of the ancient sacred Seven Fires Prophecy $W$ ampum Belt for 40 years. This prophecy, known to many peoples across the world, told of the important choices we would have to make at the time of the Seventh Fire, choices regarding our relationship with Mother Earth and each other, and that time is upon us. We have to choose wisely to ensure a meaningful heritage for all humanity and life forms. We have to choose urgently.

A global shift in our value base is essential. We need to move from greed and corporate domination to generosity and sharing; from fear and war to racial harmony and peace building; and from relentless environmental exploitation to the three indigenous Rs - reverence, respect and responsibility for Mother Earth.

We need to inspire people across the world to embrace these values urgently. This is the great challenge of our times - this is what the Seven Fires Prophecy warned about. It is the task for which we have to strengthen ourselves collectively. The transformation comes from within first; then, recognizing that we are all connected, we reach out to transform our brothers and sisters and leaders. We strive to humanize institutions.

I hold a vision for an Indigenous Centre where we can all come together to animate this future. http://www.asinabka.com/

When we come together with one heart, one love, one mind and one determination, 
we will be creating the pathway to a Circle of All Nations, a Culture of Peace. (William Commanda Archives)

The contemporary meanings of the word sacred attest to general sentiments referenced above. However, I note in WC's words, he generally used the word spirit, and he is preoccupied with a much larger integrated focus on the Laws of Nature, environment and relationality and I argue that this reflective of his fundamental spiritual priorities.

\subsection{Concluding Remarks}

I conclude by noting WC's affirmation of the Law of Nature that was negotiated via several vehicles. These included intense empirical and experiential relationship with cosmic, rock, land and water, four key elements, plant and animal life, ritual and ceremony, movement; with earth emergent language; and with ancestral epistemology mediated by auto ethnographic reflexive engagement with petroglyphs, pictographs and canoe craft. It was articulated metaphorically in his wampum heritage and prophecy, indicative of dynamic perpetual syntropic space-time evolution and renewed emergence of life. He performance mapped and articulated this constantly and insistently.

There are four critical themes observable in William Commanda's epistemology:

1. He repeatedly discussed the Laws of Nature in the context of the intelligence of nature, evidenced in the evolutionary processes fostered by those laws; this intelligence is an essential property of all organisms, of the biosphere and the ecosystems that it holds;

2. A multidirectional understanding of time (through study of the cosmic, stars, and light) impacted the prophetic, visionary knowledge of his ancestors;

3. He was an integral evolutionary component of this world, as, in Ian McCallum words on ecological intelligence, a human animal (McCallum, 2008);

4. This intelligence of nature was being denied by western science and religion, and in contravening the Laws of Nature, they were contributing to irrevocable environmental destruction and devastating loss of species. 
WC asserted an approach to life grounded in relationship with Mother Earth, and as off-spring of Mother Earth; she and all that she was linked with were therefore relations: the cosmic and stellar world, the four chief elements, fire, earth, water and air, the rock and mineral world, plant life, the animal world (inclusive of insect life, four-legged, water and winged creatures), and the unseen world. Some of this world was abrasive; it was challenging and dangerous, but it was not malicious; in the grind against the greatest of challenges, a frozen land in an agonistic process of meltdown, transformation, and violent re-birthing, ancient ancestors learned to spark life themselves; and this was the knowledge passed down. It was this ideological framework that he wanted to introduce to environmentalists. My first memory of WC's teaching pertained to water. He pointed to two containers of water, one clear, the other with 'bibbits' - minute particles; and he asked which I would drink. The clear water was dead water, he said; and thus began to teach me about the organic principle of life generated in water. I explore his extensive work for the protection of water later. His father's knowledge of the stars, seasons, waterways, fire, animals and survival in the natural world, his language and wampum heritage left clues in WC's storytelling of this knowledge of entropy and syntropy, and his dynamic engagement with negentropy. He engaged negentropically to animate regenerative effect: that is to realign cognizantly and empirically, consistent with the Laws of Nature.

The predominant factor impacting WC's way of life was his relationship with and knowledge and understanding of Mother Earth. This understanding of relationality came from knowledge passed down to him from his ancestors in Indigenous epistemologies transmitted in formal and informal communications. A range of approaches contributed to his knowledge base. He learned through language and ceremony, from the ineffable and intangible, and from direct experience and observation, consistent with empirical knowledge 
acquisition practices. He learned from his ceaseless journeys throughout his long life, Mamiwinini body in constant motion. He learned through his passion for communication, oral, multilingual, textual and visual. He learned experientially from physicality and personal, communal and environmental health challenges. He also engaged inter actively with nonIndigenous sources (people, books, documentaries and events). These factors combined to find expression in his Circle of All Nations articulation of a way of being, doing, becoming and asserting, always consistent with the Laws of Nature foundational knowledge.

With the publication of Culture of Nature, Wilson (1991) researchers examined the cultural construction of nature; for example, in discussing nature as tropos (artifact, movement, displacement) Haraway (1992) argued that nature cannot pre-figure its cultural construction through practices. While this challenges mainstream configurations of nature as material and thus available for constitution as commodity and thereby validating capitalist accumulation (Castree 1995), WC was grounded in a totally different orientation into an integrated, emergent relationship with the Laws of Nature. This belonging with cosmic nature, motional ways of seeing and understanding, and earth emergent knowledge generation practices enabled Indigenous Peoles to understand the looming global environmental crisis in a different way; as children of Mother Earth, they themselves were under threat.

I argue that his wampum heritage provides a conceptual lens into the structure of WC epistemological project, and that the trajectory of syntropy affirms the prophetic underpinnings and the motional generation of negentropy to counter entropy in a manner parallel to the law of life evident in water. As noted earlier, in the language of syntropy, water absorbs syntropy from the quantum level and materializes it at the macro level, and that this critical law of the universe, the law of syntropy, a Law of Nature, can only be seen operational in the presence of water. It affirms retrocausality, the future to past cause of effects because 
it demonstrates life force energy in its complex, cohesive regenerative, organizing structure, quite different from the entropic, energetic dissipation of past to future, cause to effect, force of causality. However, if one is alien to this life force of nature, how can one then be impacted by negentropy? WC hosted workshops on relationality, conducted ceremony honouring spirit water and profiled the science of Dr. Masuru Emoto and the Messages of Water (2002), commencing in 2002; this scientist photographed the impact of language and emotion on the physical structure of water crystals; German scientists are now pursuing this research. This understanding aligned with WC's own relational views.

Acutely aware throughout his life of the Anthropocentric destruction of his Mother Earth he sought means to reverse the entropic momentum by means of a counter-spinning of energy. This is consistent with the dynamics of syntropy. With the prophet's capacity to see the early warning signals of the annihilative consequences of entropy, he reconstituted the components of death and decay to animate reorganization and life; he was a virtuoso at this, because he accessed his knowledge directly from relational study of the source of life herself.

From the 1940s, he challenged domination of his Mother Earth as a guide of tourists, and with his work in the lumber industry. He raised concerns nationally at the 1987 Aboriginal Constitutional Debate, at the 1996 release of the Report of the Royal Commission on Aboriginal Peoples on Victoria Island, Ottawa. He spoke internationally at the 1990 Pre-Rio Earth Summit in Paris and in 1993 at the first United Nations Cry of the Earth Indigenous Gathering. He raised his concerns in his Circle of All Nations work and workshops on Sustainable Relations and Waterlife, and at the launch and roundtables of Species at Risk. Act. He advocated for the protection of American Eel, Wolf, Loon and Polar Bear. He challenged the forestry industry (on one occasion refusing to offer a blessing and welcome at a national forestry conference), test drilling for uranium and damming, amongst other 
environmental issues. He also organized educational events and workshops, and was ceaseless in raising his voice for the protection of his Mother Earth. He engaged actively with all the local and national environmental organizations, and most current leadership would acknowledge that he ignited their efforts over the past decades. Preliminary documentation of this massive, unfunded effort by one man, literally till his death at age 97 , is included in the digital atlas, and his websites.

Six years after his death, it is clear from his presence in theses and academic research, and in social media and public events, that he remains encore vivant to many. My research is focused on determining whether his approach to environment and related matters can (or does) contribute to (western) academic discourse.

\subsection{Summary of Findings}

In this chapter I have iterated the critical Law of Nature factors that intermeshed as the matrix from which WC's web of knowledge, way of thinking, way of life and performance mapping emerged. Direct interface with elemental and geo-cosmic forces from birth produced expansive spatial identification and multi-directional temporal perspective. His Algonquin name, Ojigkwanong, Morning Star articulated the direct interconnection with the three predominant time markers and life generators, and drew direct attention to the regularity of emergence from the nightly domain of Tibi Kizis, Moon, to the daily governance of Kitchi Tibi Kizis, Sun, both experientially and empirically manifested and materialized on Kitchi Jojo Aki, Earth, as his living reality. This attachment was to both an expansive and flexible gaseous cosmic net of radiant energy, motional and static stellar bodies, light/time transmission, demonstrating flux, fluidity, mutability, instability, motion, and graduated visibility and associated revelation of colour, as well as, conjointly and materially, to the concretized geo-physicality of terrestrial entrenchment. Together, they constituted the 
ontological and existential backdrop for permeability, interpenetration, transmission, transference and transformation. The magnetism of Moon, Tibi Kiæis, was experienced daily in the gravitational force exerted on the waters that governed the lives of his peoples of the eastern seaboard. This informed the foundational, motional, ontological condition registered in his name for his people, Mamiwinini, the peoples who were always in motion, who moved every day in suit with the cosmic bodies. They were also hunter/gatherers, and the quest for life entailed interface with the animal world. Their principal material artifact was the Watercraft, Canoe. It embodied the concretization of earth emergent product representative of the plant world, as well the motionality, fluidity and instability of the water world. Its motional capacity was governed by wind and current, both invisible, but tangible and evident in interconnection with materialized Earth, in particular, in her first manifestation, Rock, Asin.

Thus the geo-cosmic domain, the four elements of fire, earth, water and wind, the motionally synthesized directions, of east, south, west and north, rocks, plants and animals were the predominant factors that informed his cartographic mapping of self in ontological study of spatial and temporal geography. He and his ancestors studied the Laws of Nature directly, positioned themselves as children emergent from this world, and studied its intelligence in multiple experiential, empirical and collaborative interfacial ways, and iterated and reiterated its implications in intensive rock art episodes of creative exercise, and articulated in a vast, earth-emergent verbal, processual language. They created mnemonic wampum devices to augment the orality that governed their way of thinking. They represented their findings in identification of four key pathways to knowing. These were through emotions or feelings, through empirical, sensual physicality, through a type of intuition or sixth sense articulated as spiritual, and through a cognitive, mental pathway. The English 
words do not do justice to the vastness of the ideas embedded in WC's language. He and others articulated this knowledge in recent years in the form of a conceptual Medicine Wheel. WC concretized the essence of his approach to life, knowledge and knowledge generation, abstracting pivotal features in this teaching tool, to build bridges with a diversity of people with dramatically different world views now occupying his homeland, especially in view of devastating environmental degradation and loss of plant and animal species, accelerating climate change. He embraced and animated the principles of interconnectedness inherent to the philosophy of Ginawaydagunuc, All Are Related in all his thinking, work and outreach and in this manner, manifested the Law of Syntropy to animate negentropy.

\subsection{A William Commanda Mapping Exemplar}

An Example of a William Commanda's Mapping Intervention and its Trail of Influence

I use diverse geo-narrative methodological tools to examine the performance mapping lens into the William Commanda discourse. A passionate desire to connect and communicate lies at the centre of his Circle of All Nations work, and he appears to have reached diverse people on multiple platforms over the course of his long life. A deep desire to contribute to environmental thinking globally animated his urgent outreach. He was interested to register some of his thoughts directly and explicitly within the domain of cartography, geography and environmental studies. He engaged actively in the initiation of this doctoral research project in 2009. He said to me, "I am a just little man, trying to do big things, because life itself on earth is in such danger" (Thumbadoo/Commanda Unpublished Joint Journal 2007).

The core elements of his geo-narrative merged the ceremonial and ritual with ancient and modern technologies. In part, he transmitted the geo-narrative through wampum, drum, canoe; through active land-based animation; through a repertoire of formal communications tools (oral and written language, Algonquin, French and English); and through symbolic non-verbal devices, including the Circle of All Nations medicine wheel motif. These are the elements embedded in and constitutive of the discursive field within which his particular narrative was embodied.

I thread the following map history analysis trail in one example of the WC discourse to illustrate its relevance to understanding his performance mapping approach. WC, in his remapping of relationships, extended performance mapping to the transformational, and in his experiment, Circle of All Nations, he performance mapped the inter-cultural zone of interface, to deconstruct the land-based colonial manifesto and re-constitute it in multi 
directional emergent mapping. Thus, when he was presented with the Key to the City of Ottawa at Victoria Island, Asinabka Sacred Chaudiere Site, on June 21, 2006, he said, "I see three levels of significance that make me happy. The City of Ottawa is acknowledging someone from the City of Maniwaki; the Province of Ontario is reaching across to the Province of Quebec; and the National Capital Region, representing the entire country, is opening its heart to someone seen generally as belonging within the confines of the reserve, the Kitigan Zibi Anishinabeg" (William Commanda's Remarks 2006). He presented his vision for the Sacred Chaudiere Site on a birch bark map. Thus he deconstructed colonial maps (Harley), exercised border crossing discursive power (Foucault), assigned meaning to metaphoric devices like his wampum belts (Rundstrom 1991) and with the acceptance of the Key to the City, empowered act and artifact. With his presentation of his birch bark 'perpetually-live' being map of the Sacred Chaudiere Site (Crampton 2009), he produced territory endowed with ontogenetic potential (Kitchen and Dodge 2007), indicative of emergent mapping (Pearce 2008, 2009), anticipatory counter-mapping (Rundstrom 2007, 2009), interactive visualization (Crampton 2009), and ignited hybrid spaces and interactive networks of cybercartography and social media (Taylor1997, 2003, 2005, 2008, 2009; Taylor and Pyne 2010). This example also serves to illustrate the Law of Syntropy in present day challenges to privatization and development at the Asinabka Sacred Chaudiere Site, precipitated by WC’s 2006 performance mapping. 


\section{Chapter 5 \\ Theoretical Lens: Historical Dialectical Logic of Inquiry \\ The Quebec Welcome Kwe Beckian! Hello Stranger! - in accordance with the integrity of the Three Figure Welcoming Wampum Belt}

\subsection{Orientation into the Historical Dialectical Logic of Inquiry Lens}

Please note that in this section of the thesis, I use the names like Indian, Native and Iroquois to maintain consistency with the historical discussions and terminology used by government, Indian Affairs documents, newspaper coverage and William Commanda himself from 1850 until 1990, when Aboriginal became the new inclusive term for First Peoples. However, I note that the shift in terminology with the poststructural decolonizing and reconstructive advances of the past decades makes some of the older terminology controversial and sometimes unacceptable. Today, one notes that the terms Indian and Aboriginal are now becoming Indigenous in federal government language, consistent with United Nations endorsement of the word. Many people prefer their own tribal/nation names.

Kwe Beckian! Hello Stranger! That, as William Commanda informed countless people, was how his Algonquin ancestors greeted Cartier when he first set foot in North America in 1534. That then is the root for the name Quebec. In his dialectics, WC used his language to assert truths rendered invisible by colonial place naming and history telling. Barrett (1991) points to language as the producer of knowledge. In clarifying the roots of the meaning of the colonial name, WC was both performing his decolonizing intent, and at the same time affirming a welcome.

In view of the dominance of the colonial narrative, it is critical to contextualize this discussion with some preliminary comments about the impact of 1492 exposure to the New World in Europe. WC himself studied this. Philosophers examine the dynamics of space, time, the world, and our place within it, and influential thinkers impact social, political, economical and scientific issues profoundly. All research is guided by philosophical beliefs, philosophical issues permeate every decision in geography. Multiple references to European philosophers dominate the western academy's understanding of the domain today. According to Stokes (2002), the earliest philosophers were preoccupied by theories about 
water (Thales of Miletus); rational and irrational numbers (Pythagoras); natural phenomena (Xenophanes of Colophon); fire, earth water, strife, impermanence and change (Heraclitus); language, thought and reality (Parmenides); atoms; and movement (Zeno of Elea). But by 399 BC, Socrates has become the dominant thinker, and philosophy as influenced by him takes the dramatic turn to ethics and how one should live. He is killed for introducing such contemplations into politics; his student Plato contemplates the just society. In turn, his most influential student, Aristotle, marries ethics/virtues/values/purpose and reason to physics, entrenches the notion that to reason and further, to reason in accordance with virtues, is the natural function of man; at the same time, he introduces ideas about purpose/teleology. In the twelfth century, Thomas d'Aquinas reawakens Aristotle, and he reconciles philosophy with Christianity. A quick glance at history suggests the early preoccupation of western philosophers with earth relations was interrupted by conflict, battle and violence commencing with slavery, battles and gendered strife in Athens and Sparta, and the Greek and Roman wars. Following these times, there were the centuries of the Dark Ages, Christianity, crusades, inquisition and witch-hunts in Europe. There were also the environmental challenges of unclean waters, disease, and the black plague of the mid 1300s. In the incumbent grapplings with the darker expressions of humanity and environment, philosophy becomes preoccupied with morals.

With Copernicus, Earth reemerged in western consciousness and philosophy in the late 1400s. Copernicus was the catalyst for a new thinking grounded in the cosmogeographical preoccupations of the ancient philosophers. He returned philosophy to the investigation of natural phenomena, and the Catholic Church did not take well to his decentre-ing of religion. However, the 'death of innocence' sustained in Western philosophy from the time of Socrates, and the moral imperative (or its eradication, through rational 
science) has preoccupied the domain of philosophy since.

WC and his ancestors asserted belonging to Turtle Island, the continent of North America, since time immemorial, and from the beginnings of the retreat of the Wisconsin Glacier, moved into the American North East, leaving evidence of their presence here for at least twelve thousand years. They were preoccupied by the same original geo-cosmic issues as the early peoples of Europe, and they registered this in language that reveals knowledge of cosmic space-time realities in the heliocentric terms that Copernicus only began to assert in 1500, and Galileo as late as 1633. Unfortunately, a huge percentage of the peoples of the Americas was decimated by European diseases by the time of Cortez (1485 - 15470). Charles C. Mann projects 90 percent died by 1491 in his book 1491 New Revelations of the Americas Before Columbus; I read books like this one and its sequel (Mann 1493 Uncovering The New World Columbus Created 2011) to WC and our copies are annotated with his comments.

Our preliminary review of early maps, reports of early voyages to North America and related observations suggested that Indigenous knowledge of the peoples of the American North East influenced European geography and cartography. I suggest further that the new geo-cartographical thinking of Copernicus emerged as a result of exposure to the heliocentric cosmic knowledge of the New World reported by the voyageurs to the American North East coast. The fact that Copernicus only began to discuss the earth in heliocentric terms in 1500, and Galileo as late as 1633, and that both were arrested, inquisitioned and accused of heresy for their ideas by the Catholic Church would tend to support this argument. This knowledge acquisition could have commenced with the Vikings at the turn of the $10^{\text {th }}$ century, who transferred knowledge to Scotland and Europe; and with other voyageurs who travelled to North America from Western Europe in the 1300s and 1400s. (Initially, European travellers would have followed the Auks, the now extinct ocean 
navigating birds, according to the unpublished research of Dean Jones, WC's friend. Jones' historical knowledge and records have been derived by intense observation of patterns, elicited from an incredible range of documentaries, maps and books, lived experience in the Ottawa River Watershed, and extensive study of Algonquin Peoples and Europeans, over a period of fifty years. Her material now comprises a part of the Commanda archives).

I note that Champlain, who was born 1567, and whose grand plan was to create New France in WC's homeland, was penning his last letter to Cardinal Richelieu in France in the summer of 1635, dying a few months later in Quebec City. His journals, a part of WC's library, reveal the cartographical knowledge secured from WC's ancestors of the American North East over a thirty-year period. This knowledge of the people of the American North East was markedly different from what was known in Europe then. Up to that point in time, only what was written in the Bible was deemed true in Europe; only what was in the Bible constituted knowledge; everything not in the Bible was evil, including the new and strange people. Yet, ironically, the earliest voyages in to the New World were revealing a different reality. Here there was a different knowledge base and ideology, and a rich material world that rapidly become an injection of new life to the old world. As WC and his friend Dean Jones discussed it, travels to the New World not only presented new lands, plants, animals, foods, resources and peoples, they also presented Europe with a whole body of knowledge that was totally foreign to them. They engaged in a massive research project: missionaries were dispersed to investigate and report; new things, ideas and people were studied intensively by the church and others; explorations were funded; and much knowledge acquisition from the New World emerged in the Old World as the Renaissance.

It is not so much this generation of knowledge that is being challenged here as the horrendous repression and denial of the foundational knowledge of the peoples of the non- 
European worlds, their suppression and its reconfiguration, and their near genocide, in a massive project of colonization, germ warfare, plunder and exploitation. To WC, this constituted a dark stain on his Mother Earth and on Indigenous performance mapping. South American historian, Enrique Dussel argued that 1492 was the birth of modernity as a concept, the origin of the myth of sacrificial violence and the origin of a process of concealment and misrecognition of the non-European (Flores on Dussel 2015; and Turnbull 2007 141). Korp, a sacred landscape scholar and historian (Korp PhD Earthworks: Shamanism in the Religious Experiences of Contemporary Artists in North America 1991) notes that by 1519, Martin Luther was challenging the Catholic Church as well, and that his ideas were being spread rapidly with the invention of the Gutenberg Press, which was also increasing literacy across Europe. Knowledge from the New World sparked thinking elsewhere, and multiple other voices, such as Protestantism, Calvinism, Presbyterianism, Knox/Kirk, and the Puritans, challenged the Catholic Church. Further, Korp argues that the Papacy represented the world now as being corrupted by evil and the 'cannibals' that Columbus had 'conjured' from his voyages to the New World, in part because of the religious turmoil it was coping with. (Korp Personal Conversation August 2017). The seizure of the gold from the New World was a further benefit to the Vatican. It was critically important to the maintenance of power in face of the concurrent challenges to its domination by Henry the Eight of England, in the emerging empire-building era. (Recall that Columbus undertook his voyages on behalf of Queen Isabella, a child then, and King Ferdinand of Spain, and that Spain was the head of the Holy Roman Empire located in Rome).

WC's recitation of history in the Miskojonia song presented shortly demonstrates his acute awareness of this Columbian history. He was determined to challenge the European narrative, emancipate voices immobilized by the authoritative language of the framed 
colonial discourse, and restore them to reflective animation. His aim was to dislodge power and he expressed this by a determination to go beyond the permissions of the dominant other to occupy space, to challenge the inclusion of some and exclusion of others, as well as to demonstrate the implications and impacts of the marginalization generated by othering on environment and related matters.

In fact, with respect to Cartier's statement that he first encountered Iroquois in Montreal, WC informed me when we drove past Donnacona, that that was the Algonquin word for "I missed the animal; it got away", Hochelaga "was our old name for Montreal", and Montreal was "moneyang: many islands; these included a blind 'snai' where a stream turns back on itself and creates an island" (Multiple Personal Conversations). Thus he acknowledged and affirmed the ancient Algonquin presence on and knowledge of the land. He conducted several ceremonies on Mont Royal, in Montreal, and pointed to the lands stretching to the green mountains of Vermont as ancestral lands. While Mohawk are identified with Montreal and Huron with Quebec City today, WC described these important meeting places as part of Algonquin traditional territory in the American North East. He noted that the first encounter Samuel de Champlain had with a native leader, acknowledged by all other tribes as the head sagamore/big chief, was with Tessouat, WC's ancestor, and this happened at Tadoussac, on the St. Lawrence. He headquartered in the Ottawa River Watershed, but travelled the St Lawrence River to the ocean regularly. WC also performance mapped his response to Cartier's flag planting when he himself went to France in 1970 after his participation in the Expo 1967 events in Montreal. Here, he was called maudit savage while he built his birch bark canoe during Canada's celebration of its hundredth birthday. Further to his consideration of the Vive le Quebec Libre sentiment inspired by French President Charles de Gaulle, he took his North American Indian Nations Government flag and, somewhat 
tongue in cheek, planted it in Paris when invited to officiate at a function at the request of his friend Benoit Trichart. He was sorry to hear that President de Gaulle died unexpectedly shortly after. WC's presence in France was reported in their newspapers, as the parents of the young student who assisted with the production of his canoe documentary, Maureen Bartholmeus, reported to WC and me in 2005 (Personal Conversation, 2005).

The following discussion attests to WC's clear understanding of the rootedness of colonial history in the near genocide of his people.

Divorce from the land was one devastating way of stripping the indigenous peoples of their heritage; its destructiveness was reinforced by the Indian Act, conceived by the country's first Prime Minister, John A. McDonald (who also acted as his own minister of Indian Affairs). In 1876, he unleashed measures which were aimed at stripping them of their identity, by defining who was and was not Indian, and in that process, dividing and conquering them, destroying their political, judicial and cultural structures, and their spirit of self-reliance and independence.

The third crucial blow, and perhaps the most destructive, came with outlawing of their spiritual ceremonies. Here government attacked what the priests could not eliminate the profound and integrated spirituality that impacted every facet of their lives.

The next attempt at cultural genocide came in 1892 with the Residential School era when children were not only frequently removed from their homes and every vestige of their "Indianness", including their language, attacked and undermined, but they were also violated physically and sexually. Sixty years later it was the time of the "Sixties Scoop", when the families were further eroded as native children from across the country were forcibly removed from their homes and housed with white families. In 1968 there was the White Paper, designed to further erode the status of the First Peoples, and eliminate the federal government's responsibility to them. (Thumbadoo Learning from a Kindergarten Dropout 2005 38)

WC viewed the history of colonization and colonialism as inextricably linked with the near genocide of his people and with the environmental crisis of his Mother Earth. He considered it critically important to deconstruct, dismantle and challenge the embeddedness of environmental discourse in a contemporary global reality informed by western force, science and thought domination. Thus, he challenged the construction of nature as established in a series of colonial discursive, social, technical and institutional practices 
whereby it is made visible and available to forms of instrumental reason as constituted by a exploitative imaginary of ownership, power and domination (drawing here in part on the language of Braun 1996), seeking instead to entrench its reframing and reconstruction consistent with his passionate assertion of his relational Law of Nature construct. His discourse emerged from cosmic space and time construction, suspended in a particle net of the unseen, the partially visible, and the tangible and the concrete, an understanding and integration of which he viewed essential for a regenerative revolution. This chapter, then, focuses on an exploration of the historical factors which underpinned and influenced the diverse ways WC framed his discourse to defend Mother Earth, and by extension, all her creation.

Indigenous peoples, including WC himself, drawing on their relationality with a living, agentive Mother Earth, had been alerting the United Nations (UN) to a looming environmental crisis from the mid nineteen forties. However, little attention was paid to the Indigenous voice until 1993, when the UN hosted the first conference of Indigenous Peoples. It was only in in September 2007 that the UN Declaration on the Rights of Indigenous Peoples was endorsed, but the Canada, the United States, Australia and New Zealand, all countries of colonial roots, withheld support till 2011. WC was indefatigable in his efforts to entrench an understanding of critical environmental concerns from an Indigenous perspective. The poststructural and post-Harlein launch of critical cartography, the decolonization of the European map, and critical geography can serve to contextualize an understanding of WC's own performance mapping, ideographic messaging and decolonizing project, drawn from the foundation of Indigenous cosmic and planetary knowledge and conviction of rootedness in Mother Earth.

His own study of history and his wampum heritage informed his stand. Even as he 
discussed the Three Figure Wampum Belt, with its symbol of the Vatican affirming a sacred agreement of equitable sharing of land, resources and values, he questioned how honourably the priests and missionaries interpreted on behalf of the Algonquins in the negotiations with the French and English. He was painfully aware that every high mountain over looking a river or several converging rivers on the land throughout Quebec, New Brunswick and other parts of the American North East, places of spiritual importance and vision quest to his ancestors, was now dominated by churches. He commented on this frequently on our road trips. Recall also the critical messages of the Seven Fires Prophecy. The seven prophets had described the major transformations that were to come into the lives of the people over different epochs of time and they had warned that the newcomers could be enemies concealed under the guise of friendship. Further, the prophecy warned that a headlong rush into science and technology could lead to a damaged and seared earth. The prophets indicated that the people would know if the newcomers came in a false way if the waters become unfit to drink and fish unfit to eat.

As carrier of the Seven Fires Prophecy Belt, WC knew that one response of the newcomers to his homeland could have been to build genuine friendship, and to have respected and endorsed the values of respect for Mother Earth and all her children of the Indian in the centre of the Three Figure Wampum Belt. However, he noted that the poisoned rivers and destroyed lands anticipated by prophecy confirmed the misgivings and warnings of the prophets. WC knew his people had been deeply betrayed by the choices of the newcomers. However, he also accepted the fact that his ancestors of the Eastern Seaboard had welcomed the new people to their continent, even while acutely aware of the risks, and he, as wampum carrier, was committed to confirming and reaffirming this welcome. 9/11 was a critical marker in the context of the Seven Fires Prophecy. The last decade of WC's life was 
dedicated to a massive negentropic effort on behalf of all. (This included on-going work on his global legacy vision for the Indigenous Centre at the Asinabka Sacred Chaudiere Site, the geographical location known by his ancestors of ten thousand years as the critical centre: www.asinabka.com).

He stated repeatedly that "My ancestors, recognizing that we are all connected, welcomed the newcomers to North America, agreeing to share our land, grand natural resources and values equally in the spirit of friendship, consistent with the guidance of our ancient prophecies, in order to sow seeds for the global future" (William Commanda Personal Archives). Despite his acute awareness of the centuries of oppression of his people, and the critical need for Indigenous decolonizing research, approaches and priorities, he was also determined to inspire a Circle of All Nations of inclusion and co-creativity. He used this vehicle as a bridge-building mechanism, grounded in his Indigenous ideology or web of knowledge, to entrench the centrality of the voice and place of the First Peoples of the land. This video link presents his discussion on such peace building https://youtu.be/zmYs2PtPc4w (2005 interview with Jean Trudel, Cercle de Paix, an organization inspired by William Commanda, now accredited at the United Nations. As such, he was not so much concerned with self-government, segregation of Indigenous issues and separate pathways for Indigenous Peoples as he was determined to assert the critical importance and relevance of Indigenous earth-based values to guide everything and everyone on Turtle Island, because he believed this was both the right and responsibility of First Peoples and was also of accelerating global importance.

From a WC perspective, there could be no separation of the environmental issues from colonial and contemporary history, and the survival of Indigenous Peoples. This informed his historical land based narrative and is inseparable from his environmental 
stewardship work of forty years on the national stage, since the 1987 Constitutional Roundtable on Aboriginal Issues. Here, for the first time, he presented the idiographic wampum law publicly in his historic narrative, and his most passionate statement called for the urgent need to address the evident and accelerating environmental catastrophe, in his words, before the end of time.

\subsection{Theoretical Frame, Methodology and Tools: Historical/Dialectical}

For this section of the thesis, I selected a historical/dialectical narrative logic of inquiry as the entry point into the examination of the principal Indigenous, performative and textual features of William Commanda's historical life and narrative. Such a dialectical process involves a discourse between two or more people holding different points of view about a subject while wishing to establish the truth through reasoned arguments. WC was masterful in this reasoned exchange of ideas around issues of disagreement, and this is discussed in the context of the historical meeting of the Indian Traditional Chief, William Commanda, with the then new Prime Minister, Pierre Trudeau, launching Canada into its hundred and first year in 1968; it was a decolonizing moment. This dialectical reasoning also informed WC's engagement with others: inmates, Iroquois, government, corporate, environmental and community voices. Since WC's pivotal position was that only he as Indigenous was 'at home' and he was intent on defending his Mother, Earth, he performed an integrated political/ecological epistemology and ontology.

As Linda Tuhiwai Smith states, Decolonizing Methodologies is concerned with the "context

in which research problems are conceptualized and designed, and with the implications of research for its participants and their communities. It is also concerned with the institution of research, its claims, its values and practices, and its relationships to power. It has 'talked 
back to' and 'talked up to' researching as an institution of knowledge that is embedded in a global system of imperialism and power" (Tuhiwai Smith $2012 \mathrm{ix}$ ). Colonialism is a complex and multifaceted type of oppression manifested through manifold economic, ideological, cultural and assimilative processes of subjugation. Scholarly definitions of lived experiences of colonialism are extensive and complex, and have informed our thinking, but are largely beyond the scope of this analysis. But it is critically important to understand how, for example, Mohawk scholar Taiaike Alfred frames the issues: "The problem that we have inherited in this generation is our disconnection from what it is to be Indigenous. This problem has been framed in many complicated ways, but really, what is colonization if it is not the separation of our people from the land, the severance of the bonds of trust and love that helped our people together so tightly in the not so distant past, and the abandonment of our spiritual connection to the natural world? ... Now, then, and forever, the fight is for the land. The land and all it has to teach, to give, and all it demands, is what it means to be Indigenous" (Alfred in Simpson 2008 9,10). This recent academic publication, Lighting the Eight Fire, The Liberation, Resurgence, and Protection of Indigenous Nations, presents the thinking of young Indigenous academics who, as the title suggests, are bringing new voice and agency to the heritage of oppressions.

I make special mention of a researcher, Eric Smith, who examined WC's discourse over the course of multiple years, scrutinizing his land-based epistemology on forgiveness, correlating it with a geographic research project on decommissioning dams, and thereafter specifically examined decolonization in the context of WC's Asinabka (love and hope) project, Circle of All Nations people (forgiveness) and and land (emancipate the waters) discourse. He examined the epistemic disobedience of Latin-American decolonial scholars Mignolo, Quijano, Dussel, Escobar and Loomba who interrogated colonialism as the 
forcible takeover of land and the restructuring of non-capitalist economies to fuel European capitalism, not only a trans-historical impulse to conquer but also an integral part of capitalist development. He finds that coloniality refracts the heterogeneity of human relations into discrete objects, empties the world of ecological relationships, and then reconstitutes a closed world according to a colonial order of power (Loomba 2005, Mignola 2000 and Escobar 1995, 2008, 2010, in Smith 2011 5).

The decentering of modern knowledge paradigms has been accomplished in part by the efforts of transnational Indigenous activist networks, global movements such as the World Social Forum, critical subaltern pedagogy, and the contributions of Indigenous elders, knowledge and wisdom keepers, and scholars. Many Indigenous Elders and scholars from South America participated in WC's Circle of All Nations events and an Argentinian organization FANCV with intervenor status at the United Nations advanced attention to the Asinabka Sacred Chaudiere Site project to the United Nations Human Rights Council's Expert's Mechanism on the Rights of Indigenous Peoples in 2014 (documents on www.asinabka.com). WC's use of his Condor feather was not without agentive power.

The insurrection of knowledges produced through ecological, inter-subjective relations disturbs the foreclosures of modernity/rationality by introducing organic, open, and liberatory political possibilities; articulated, endogenous practices of economic, cultural, and ecological alterity and deliberate counterwork result in reclaiming power (Escobar 2010 in Smith 2011 9). In doing so, community-based social movements challenge and weaken (with varying degrees of success) the structures of universality, unity, totality, scientific and instrumental rationality, and the domination of nature.

Smith also examines the rationale for the use of narrative to deconstruct colonial constructs. Citing Battiste and Henderson, he notes that storytelling is a critical vehicle for 
the transmission of knowledge of life, land, and ecological relations within Indigenous ontologies (Battiste and Henderson, 200010 in Smith 2011 13). An Indigenous anti-colonial and decolonial political ecology is narratological. Tuhiwai Smith (1999) stresses how necessary it is for Indigenous researchers to "demystify knowledge and information" and speak "in plain language to the community" (Tuhiwai Smith 1999 161). Thus storytelling, with detailed "thick" description, and analysis of practices are justified to deconstruct and rupture the dominant colonial narrative, the colonial encounter and the neocolonial insurgence, and reconstruct inter-subjectivities and ethics, privileging alliance and community over difference and factionalism.

This was the ontological package that William Commanda resisted, and the immanent hope was entrenched in his spiritual groundedness, inclusive of the wampum heritage.

\section{Methodological Tools}

WC was born in Kitigan Zibi Anishinabeg. Originally named Lac Desert, the Reserve was created in 1853 after WC's great, great grandfather Pakinawatik, made three trips to Toronto to negotiate its creation. He envisioned it as a sanctuary to preserve the language, culture and lifestyle of his people who, already decimated by colonial disease and warfare, were now under tremendous pressure with the arrival of settlers to the Ottawa River Watershed. These people were destroying their waters, trees, hunting and trapping grounds, mobility and lifestyle. However, by the time (1867) of the creation of the provinces of Lower Canada (Quebec) and Upper Canada (Ontario) and the Dominion of Canada, and the creation of the Indian Act, lumber companies (Gilmour, Hughson and Edwards) were already tree cutting in the Limits, which comprised about three quarters of this first reserve in Canada. With the formal creation of the colony, the developers, priests, Indian agents, police, 
game wardens, and fur buyers assumed repressive control of the lives of the First Peoples, an early violation of Pakinawatik's intent behind the securing of the lands to be reserved for the Indians.

This case study of the narrative of one singular man over the course of a century of significant global temporal and spatial change examines the inseparability of his personal land-based story from the conjunctures, contingencies, contradictions and duplicities emergent from historical times of contact into the present. I examine his contemporary presence in a colonized Europeanized world rendered both globally connected and yet violently divided, with ideologies of duality rendered manifest from the time of his birth with the World War One, (so different from his ideological foundational embeddedness in Ginawaydaganuc interconnection), and a world in constant transition on multiple planes over the course of his own lifetime. This is a world grappling with global citizenry and tremendous diversity as well as crises of enormous scale, (environmental, climate change, extreme weather, wild fire, war, racism, immigration, refugees, mental and physical health, megacities, drug abuse, crime, and, rising and plummeting birth rates), while still dominated mainly by Western capitalist, neocolonial and neoliberal ideology.

I contextualize this examination within the much larger idea of Ginawaydaganuc-All is Connected. This is the all-encompassing word William Commanda used constantly, affirmative of his belief that all are inextricably connected, both people and all else in the cosmic world we inhabit, contrary to the dualistic and divisive understandings that dominated his times. Ginawaydaganuc celebrates diversity as the principle creative force in evolution. I argue that WC created a discourse around this principle, and his informally constituted Circle of All Nations is a global eco-community (comprising thousands of people who participated in his annual gatherings; governments and non-government organizations and citizens at large with 
whom he interfaced; people he met on his decades of global travel; the other thousands in the cyber world; and people who are coming to his ideas since his death) to animate this mindset in their varied individual pursuits, in emergent and evolutionary ways. His effort is augmented by the earth-based resurgence of Indigenous presence around the globe, particularly since 1987, popularly called the time of convergence. I argue that, fully aware of his colonial history, he saw that its trappings impacted all detrimentally. He therefore determinedly animated the Circle of All Nations through his Anicinabe articulation of the Laws of Nature in a conceptual framework insistent on inescapable relationship. I argue that he was both worldly and spiritually grounded, multifaceted and focused, humble and compassionate, and entirely self-propelled, and that these and other unique characteristics contribute to the narrative and the scale of the reach of this single person, whose ultimate goal was to awaken people to their relationship with Mother Earth.

In addition to the singular library of archival material that WC collected (a partial list is included in the thesis), commencing with the 1937 National Geographic publication that featured the native tribes of North America, final drafts of scholarly research projects, and slides, audio and visual materials, he also engaged in extensive storytelling with me. I made notes on the computer, and scraps of paper at every turn, and audio or video recorded him when possible. This included the daily review of local and global news. By his repetition of certain stories, I grew to know which ones were particularly significant to him and which I needed to research further to better understand the contextual background of his thinking and his narrations.

I employ a dialogic approach to writing here, recognizing that research and writing are relational. Thus, in this sense, the research is informed and constructed by the writing, as the writing is informed and constructed by research. Reaching beyond this direct relationality, 
there is also the unique and privileged relationality to the articulations of a brilliant statesman and thinker, who wished to leave a recording of his narrative. Finally, there is also the relationality to a wider set of social, temporal and spatial factors, land-based, historical and academic. WC and I travelled constantly, between Ottawa and Maniwaki, across the American North East, and internationality. The body-in-motion pedagogical space also contributed to the retelling of the historical narrative, because the land itself brought certain narratives to the foreground and positioned them agentively. Our thousands of photographs provide graphic representation of land-based history and geography of the land.

Finally, I note the inclusion of other voices in the National Film Board interviews for the creation of the documentary, Ojigkwanong. I contextualize this storyboard framework with detailed description to permit extrapolation of patterns and priority issues, and to present independent evidence to substantiate some of my findings.

This leads me to comment on Foucault's ideas. I am conducting a discourse analysis of WC's approach to environment and related matters, but I am not engaging extensively with Foucault's approach. However, I refer to his ideas about genealogy, and the exploration of the processes that contribute to the constitution of a person's subjectivity. In this consideration, he was alluding to matters beyond the idea of the Cartesian subject, an individual, unified self with agency and control over itself through thought and reason. He was referring to the genealogical accounting of knowledges, discourse and objectivity emergent from a larger contextual background. I suggest that the detail in my unpublished records of WC's narrative offer rich material constitutive of such a genealogical backdrop. Furthermore, this is unique material, since despite moments of despair and disappointment, WC did not construct himself as victim, and his astute native alertness to seen and unseen things contributed to his virtuoso performance as narrator. In fact, even with respect to the 
terminology of subject, we ourselves discussed the word in the associative contextualization of the sovereign. With his notion of a land based sovereignty, he did not ever see himself as subject, as his work with the North American Indian Nations government, and his 1968 discussion with then newly elected Prime Minister Pierre Trudeau attest. This takes academic notions of subjectivity, when associated with sovereignty, to a new reframing of power beyond the introspective.

WC's life was a conscious decolonizing and reconstructive project. I explore specific components of the William Commanda discourse and travels over the course of his long life (1913 - 2011). Key documents and video material in his library and archives, created throughout a century of tremendous change, provide a rich record for research and analysis. My unpublished notes of WC's dictations are dense with detail and nuance. Extensive collections of textual, archival material, books, audio and video recordings, photographs, artifacts (e.g. canoes) and the memories of many other people of tremendous diversity also provide the background data for the analysis. In addition, I remain in regular contact with his sister, daughter and son-in-law, his nieces, his Circle of All Nations colleagues, and many of his old friends, including his oldest friends, John and Shirley Garren from Pennsylvania, and other Americans who established a fishing and gaming club on his father's and grandfather's land at Lac Desert, Quebec. During his youth, he was an activist challenging the environmental abuses and excesses of tourists. As an adult, WC became acutely aware of the hegemony and oppression of colonial domination, and actively challenged this with his extensive work with the North American Indian Nations Government. This chapter examines his decolonizing project from a historic lens; this is inseparable from his environmentalism.

Over the past several decades, literally thousands of people from across the globe 
became a part of Grandfather William Commanda's informal Circle of All Nations eco-peace community and animated the multiple aspects of his work priorities in individual ways consistent with their own passions, commitments and capacity. In this way they grew into deeper selves; and this is why their voice and presence keeps Grandfather alive. This section explores how he moved from historical challenge to transformative relationship-building.

The four Commanda-specific methodological tools used to examine the historical narrative include the National Film Board documentary, Ojigkwanong, evidence of Indigenous leadership presence, the Lament commentary, and several Circle of All Nations pedagogical exemplars.

\subsection{Conceptual Medicine Wheel Orientation Map}

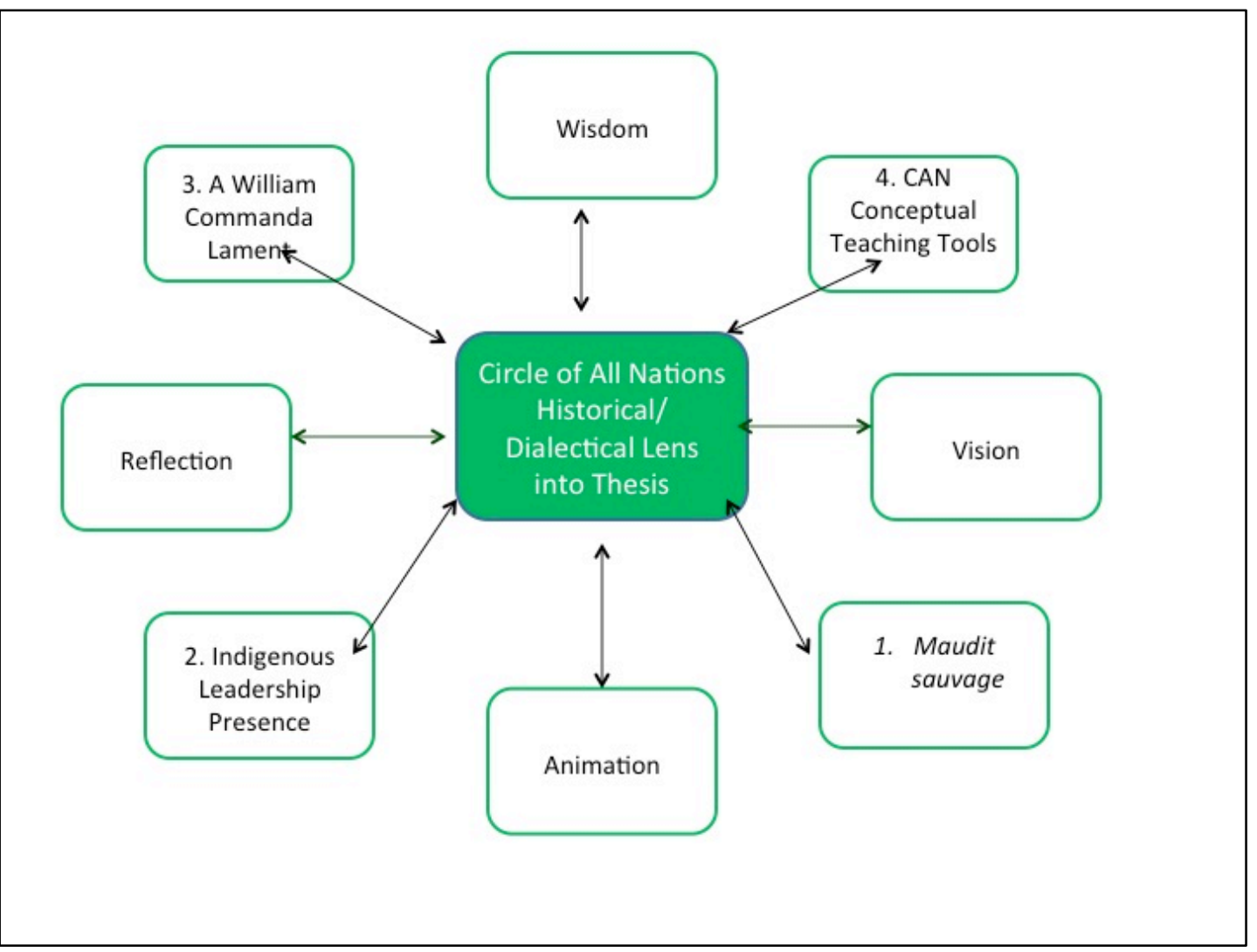

Figure 5. Conceptual Map of Circle of All Nations Historical / Dialectical Lens into Thesis 


\subsection{Discussion, Analysis and Observations}

WC could see the overt and systemic oppression of his peoples clearly, but his immersion in the spirit of Ginawaydaganuc meant that he also learned to embrace people of all backgrounds, Indigenous and non-Indigenous. Never idle, WC also had a tremendous zest for life, within which it seemed a much larger life force was at play. In view of his study of colonial oppression, excessive and destructive resource extraction, and decades of attunement to the looming environmental crisis, he determined that he had to make newcomers and all others respectful of his land. As the years passed, and more newcomers arrived in his homeland, this drive became increasingly compelling. I argue that he very consciously developed an inclusive discourse to redirect people to relationship and thus responsibility to Mother Earth.

Four shifting narratives shaping WC's story trace this trajectory in his discourse:
a) Maudit sauvage/Dirty Indian
b) Respected canoe builder
c) Noble savage, and
d) Virtuoso performer

\subsubsection{The First Narrative - Maudit sauvage}

Maudit sauvage/Dirty Indian - the historical presentation of the First Peoples of the continent, created by the doctrines of colonization and imperialism, now increasingly challenged and discredited; the digital age, internet, Wikipedia and social media are accelerating the re-telling of history

Here, I draw on the NFB documentary, Ojigkwanong, primarily located in a federal prison, to scrutinize certain aspects of WC's historical narrative. The story thread leads from his childhood of earth-based learning, to fight for Indigenous rights, to advocacy for environment, to forgiveness to ensure environmental stewardship.

In 2000, WC hosted the Circle of All Nations Millennium Peace Gathering in Ottawa. The National Film Board documentary, Ojigkwanong, Encounter with an Algonquin Sage, by 
Lucie Ouimet, was premiered at the Canadian Museum of Civilization. It included interviews with him in his home at Bitobi Lake, and documented one of his visits to La Macaza Federal Penitentiary in Quebec. (WC made a point of engaging regularly in outreach to federal prisoners in the province, as part of his social justice work). The documentary was produced in French, with English sub-titles. This underlines the scope of his oral communications. Also, French was WC's second language and the English translation of his chosen words only approximate his meaning. I draw on this version of WC's story for the consideration of this first of the four narratives; it alerts one to the cosmic space/time reach of his historical narrative with a particular group of confined people, Indigenous offenders. The discussion that follows draws on elements of the script to examine the issues that underpin and inform WC's environmental perspective in pentimento fashion.

\section{a. Geo-Historical Narrative in Ojigkwanong Documentary}

Discussing nature as his school, WC asserted that he learned everything from his father, Alonzo Commanda, who was representative and illustrative of the deepest interface with Mother Earth and the Laws of Nature. As noted earlier, he had an extraordinary knowledge of the natural world; from WC's stories, I learned that he was one of the last of the people who could read the stars, fire, land, waters, wind, weather and climate so masterfully. During his first meeting with WC in 2007, historian archaeoastronomer, Dr. W. Sullivan, himself an expert on cosmic calendars, realized from what WC had shared about his father's stories, that Alonzo Commanda had known full well the significance of June 21 as mid-summer. The Best Guide in the World article (John Durant, Saturday Evening Post, December 23, 1944) provides tangible evidence of his superior environmental knowledge.

WC himself learned to understand the bush and trees very well; and when he was working as a guide in 1952, he took tourists out moose hunting. Two worked in the lumber 
industry. ${ }^{3}$ The men asked his opinion about the potential of two birch trees as veneer logs: he pointed out that one was still green and the other knotty, and they hired him on the spot. That was when WC became a company man. He took the job as veneer log buyer and worked hard to entrench log selection practices that would not destroy forests amongst the tree cutters and companies. WC worked with a Dwayne Cole, who taught him to read, write and count while they were away from home and working; WC was about 40 at the time; his counting accuracy became legendry in the company. Dwayne Cole took him to Jay Peak in Vermont. At Jay Peak, they were skidding logs with bulldozers, and destroying the trees and bush. WC advised Cole to change this to the deployment of timber jacks and horses. WC formed such a strong relationship with the white American, that when they were both widowed, Cole invited WC to live with him. I describe this anecdote to point to the unique style of education, knowledge transmission and environmental stewardship, history, and equality and relationship embodied in WC's story. These and more constitute the referential materials embedded in his discursive narrative (Thumbadoo/Commanda Unpublished Joint Journal 2007).

Our notes on Oppression: Indian Agents, Priests, Game Wardens, and Fur Buyers: Cruelty, Confiscation, Cheating and Civilization (Thumbadoo/Commanda Unpublished Joint Journal 2007), regarding the tremendous challenges and traumas WC and his community experienced during the past century contextualize the intersectionality of the personal, historic, political, environmental, economic and religious dynamics that infused and informed his evolving philosophy and framed his teachings. From his earliest childhood,

\footnotetext{
${ }^{3}$ They were working for Blair Vermont Plywood, then it joined Owens Glass to become Owens Illinois Plywood, then the company became Roddis Veneer Lumber, and then, as WC put it, 'they merged and got bigger and climbed up to become Weyerhauser.' By the time he wrote to them about his vision for the Asinabka Sacred Chaudiere Site in 2009, they had 'become' Domtar. We likened this manner of operations to the Pilsbury Doughboy, where the corporations all look the same and real, but when you poke, you cannot find the backbone (Thumbadoo/Commanda Unpublished Joint Journal).
} 
WC witnessed the control of the Crown, and their reign was 'cruel', and he saw the ramifications impacting all aspects of the present-day struggles in native communities. As noted in the documentary, with the implementation of the Indian Act, every aspect of life was controlled by the agent, and Indigenous leadership structures undermined and decisions were regularly vetoed. During this early period, the forcible suppression of the wampum governance heritage and its replacement by the colonial regime was enforced by the Royal Canadian Mounted Police.

In the documentary, WC talks about near starvation during his childhood. The traditional practices of the hunting and gathering peoples was forcibly disrupted by colonial powers and agents. When WC was under ten, he recalls that his father had hunted a moose which was to provide for his family's needs while he was off in his trapping grounds over the period of several months; this was during the severest part of the winter, when not even rabbits were visible; (as an aside I note that his wife Mary Commanda talked about the five seasons of a year; this time of Jack Frost was one of them). The moose was confiscated by the game warden. Community members were put in positions to spy on each other and report on infractions of the new rules of the colonial authorities, social structures were fragmented, family stability violently eroded and long ranging distrust, manifesting to this day in reserve communities, was instigated by these practices. Note further that this was around 1920, the years of the First World War, and the great depression was approaching. When things got really challenging in Canada, there was welfare for the townspeople of Maniwaki, but not for the Indians of the reserve. The provincial government, which assumed control of their lands and resources, accepted no responsibility for their survival. Further, they confiscated fishing nets and canoes. WC recalled this happening with his Uncle John Cayer's handmade cedar nets. WC's niece Daisy Commanda Jacko related that when 
her father, in some moment of desperate need during the depression, approached the Indian agent for the help Indians were entitled to, he was so denigrated and humiliated, he swore never to do that again. The priest's input to the Indian agent, played a role in this, because her father had challenged the church regarding the burial of a relative. Thus the hostility of the authorities became multiply constituted and intermeshed. The self-sufficiency of a people who had learned to survive for centuries under exacting climate changes was severely undermined by near genocidal colonial practices.

Another story I share is about WC and his father approaching their winter cabin one year to see smoke rising from the chimney. Thinking that one of their fellow hunters was warming up the cabin, they approached to find two Frenchmen waving a paper from the provincial government giving them rights to Alonzo Commanda's trapping grounds and cabin. That night, he returned with friends and trenched (killed) all the beaver, even the young ones; normally, beaver colonies were only culled of a certain number of adults. He was a master beaver man. He had been goaded into doing something contrary to his understanding of the Law of Nature and his spirit, and it impacted his life destructively. In one of our conversations, WC and I discussed the beaver in the context of the original sin of the Indian: the trading of animal skins for European goods. This perhaps was reasonable enough initially, since the flesh would have been eaten, and skins accumulated; but the killing of beaver as a commodity was another matter. The beaver had a special spiritual and mythological association with release of land-locked waters, the creation of the Great Lakes and land, and recognition of its role in this life creation process itself was recorded in the stories, legends and pictography over the course of centuries (Sullivan 2011 in William Commanda Personal Archives/South March Highlands challenge). With the killing of beaver as a commodity came the anihilation of spirit, nature, self and history; generations later, as 
the killing continued, this led to the near extinction of Canada's iconic symbol and Nature's primary water engineer.

Perhaps it was the embodied knowledge that there is much beaver blood to assuage, and his childhood exposure to the consequences of revenge, that inspired in WC to the exploration of pardon and forgiveness. But this was not before this relentless oppression of his peoples at every turn drove him to much repressed anger. It was only when he was declared terminally ill with cancer (in his words, condemned to die), and then had a transformative spiritual experience, that he came to terms with this history and then revisioned his own life, in his personal negentropic experience.

As noted in the Ojigkwanong documentary, when WC reflected on the Indian Agent's statements that You people will never get a school, and you'll never have Indian police ... things like that ... because you're not civilized, he concluded, from his experiences, that for me, civilization is minding your own business, (that) is civilization. When you invade others, with no right, taking them by force ... if that's civilization, it's not my understanding of it (Ojigkwanong NFB Documentary 2000).

The interesting thing is that despite this capacity to see, as it were, into the Heart of Darkness, WC nonetheless also saw shades of grey, and managed to maintain relationship. Each of the three most reprehensible Indian agents of his times ended up with mental illnesses; WC pointed this out repeatedly. It was a significant part of the message he wanted to impart, regarding (and alerting people to) the karmic consequences of choices and behavior. Yet WC visited them in hospital, relieving the wives of some of their visitation duties. A monk taught WC to sing acapella. He engaged in construction projects with the priests, and took them hunting.

An undated handwritten note adds an interesting clarification about WC's thoughts about a significant evil: "If you go to it, it does not mean that you are for what they want; 
you can still be friends. But ownership - that's what's wrong today". Indians were the only people who did not create money, he stated frequently. Their values were grounded in sharing and generosity. (Thumbadoo/Commanda Unpublished Joint Journal 2007).

With respect to the fur buying industry, multiple practices ensured that the Indians were cheated and rendered impoverished and powerless by the colonial system. They were prevented from feeding themselves in traditional ways. They were paid in kind, with the flour and sugar of the white man. They never brought in enough skins to match the prices of these diabetes-inducing goods during the depression; thus, they would owe skins from the next year's harvest. Not only would they then be working from this deficit position, but the skins would be measured by gun length; and each year, the guns would be bigger. I recall taking WC to a flea market in Stittsville, Ontario, a few years ago, and a Jewish dealer had a muskrat hat for sale; WC wanted it, but the man wanted too much money; WC deemed it worth only so much money, and that is all he would pay. Eventually, the man sold it to WC at his price. The exchange, as the dealer probably knew intuitively, was actually about settling a different score.

b. Never Was Idle Activism: Supreme Chief of the North American Indian Nations Government

Huron activist, Jules Sioui (1906 - 1990), was critically important to the reassertion of Indigenous rights and voice in North America and is the architect of the North American Indian Nations Government, the critical precursor to the Native Indian Brotherhood, now Assembly of First Nations. Note the following discussion.

Prior to the time of the creation of the United Nations, during the war years, Algonquins and other Indians took their grievances with the colony to the evolving League of Nations in Geneva: they collected money to enable Mohawk Chief Descayer to travel and represent them there in the 1920s. Jules Sioui, Huron activist, was working in New York, attuned to global political deliberations, and he became actively involved in political action on Indigenous issues. He took Indians, including Algonquin Chief John Chabot, to the 1945 meeting at the United Nations, met with the Secretary General Trygve Lie and asked by what 
legal right the immigrants were occupying their seats; he was referring to the President of the United States and the Prime Minister of Canada; Indigenous activism had reached new level of assertion. By 1946, WC was involved in the effort to restore Indigenous peoples to their sovereign birthright, and he and Indians from Canada and the United States became involved the organization that Jules Sioui created, the North American Indian Nations Government, created in part to assert their hunting and fishing rights. WC became his most active partner, and in 1953 was appointed Supreme Chief. Each week, winter and summer, in his own work with the lumber companies, he drove to New Brunswick and other places along the St Lawrence and into the States. He overnighted with Jules Sioui and his wife in Huron Village, Quebec City, and they developed the plans for the organization. During his travels, he awakened Indigenous individuals and communities to potential and promise. A poster for the 1960 Annual General Meeting affirms its 15 years of operation, and WC's leadership for ten. A serious study of its ground-breaking work of two decades to arouse Indians from their abject poverty and oppression as wards of an abusive state machinery is warranted. While we have extensive documentation and newspaper coverage of this history, we were not able to find Jules Sioui's name in the library of Indian and Northern Affairs Canada. Jules Sioui was imprisoned twice for this rights-based work, which asserted Indigenous and Metis hunting and fishing and travel rights, affirmed naturalization (that is all immigrants could choose to become a part of this government), challenged conscription, and man-made protocols; and, consistent with the Three Figures W ampum Belt ideology, centered the Indian in his country. His 1949/50 prison term included a seventy eight day hunger strike. WC worked hard with a Mohawk colleague to get the by then very frail activist out of jail. Ironically, Jules Sioui's own appeal had exonerated him in the court case, but he had been detained in prison by the corrupt judicial system. The hunger strike left his body and spirit broken. I have just uncovered, a five page letter that Willliam Commanda wrote (and typed himself) on October $1^{\text {st }}, 1972$, to Mr. Irving Perley, Tobique Reserve, in obvious response to his query. It provides a mini history of the work of the North American Indian Nations government and of WC's astute assessment and indictment of the interventions of government. It is a powerful example of his detailed narration, research and deconstructive capabilities, and I quote it in its entirety:

True Copy

October $1^{\text {st }}, 1972$

Mr. Irving Perley,

Tobique Reserve,

Perth, N.B.

Since I have been very busy with students from the University of Ottawa, I had almost forgotten your request of wanting me to write you a letter to reinstate my position as Supreme Chief of the North American Indian Nation government "of North American", which is very big and vast just as what you said.

First of all, what you will have to do is go to Quebec city where they keep all the judgements of the proceeding. You will find in 1949 -1950 the federal government vs Jules Sioui; there was a court held in Quebec city. In the first instant, the English judge (Gibson) ordered the jury or instructed the jury to declare him (Mr. Sioui) guilty. Two weeks after, the secretary appealed to a higher court and again it was held in Quebec 
city. The only difference was instead of the jury and one English judge, there were five judges and thus Jules Sioui was favoured, and then was acquitted.

Jules Sioui was tried again for supposedly trying to incite the North American Indians to rebel against the crown, and the whole structure of capitalism and colonialism, prior to the court case in Quebec City.

Jim Horton from western part of north America was appointed was first Supreme Chief of the North American Indian nations (1943). Then two years later he was unable to attend the convention for various reasons. He resigned the post for what I thought was too much pressure from the puppets governments.

Then James Fox from Muncy Ontario was appointed as Supreme Chief in 1945 and in 1947 he was forced to resign by ill health, and also from lack of funds. During the convention in Detroit Michigan in 1947, James Fox declared he will have to discontinue and gave the two above mentioned reasons.

Then Bernard Commanda was appointed by the North American Indian chiefs that were present for the people and by the people of North America in 1947 and by appointment to be Supreme Chief of North American Indians for the first time, one who was appointed who was very close to Tecumseh and to Pontiac who was a Ojibwe from Georgian Bay, Ontario and one who would not take bribes from the European immigrants and this was where Canada and Washington began to take notice, especially, after Supreme Chief Bernard Commanda and Secretary of North American Indian nation Jules Sioui went to the United Nations in New York city and accompanied by Chief John Chabot from Maniwaki, Que. And at U.N. they were received very warmly by Secretary general of United Nations at that time Tri Vye Lee. The secretary and Supreme chief had $2 \frac{1}{2}$ hour conversation with the Secretary general of United Nations of the world for the first time in histories of North America. The worlds Secretary listened with interest and asked the Supreme Chief and secretary for North American Indian Nation to supply him with the documents stating what was said during the $2 \frac{1}{2}$ hours meeting and shortly after this the United States and Canada were told by the Secretary General of United Nations (Tri Vye Lee) from Swiss that these two countries did not have the right to occupy the seats they were holding at U.N. assembly.

You can imagine what took place afterwards these two puppet governments, United States and Canada. They couldn't afford to lose what they had established (e)illegally, all the riches etc. etc.... They had to do something and do it fast because the organizers were not sleeping they were going 100 miles and hour and pushing ahead day after day. So the puppets did the only thing they can do: "they got rid of United Nations Secretary and make a case against the Secretary of North American Indian nation convicted him for telling and putting Indians wise to their rights and as possessor of rights.

The puppets did not and will never have the rights to form a legal government in North America. So long as the Indians will join hands as one nation and fight those two puppets with legal means. But you know we don't have and didn't have time to 
establish Indian tribunal of Indian courts, but we were getting there bit by bit every year. However dragging us to their Kangaroo Court, they opened the door for us to tell them things they did not want to hear, for instance forces do not have rights only bandits use force and we stressed that the puppets were using force and not rights to rule over us.

After a long battle and $\$ 35,000.00$ spent to defend the rights of the Indians and what I am saying about moneys being spent to recover and maintain the rights of the Indians was sponsored with pride, by the Indians themselves, and after the court, some months later, to be precise September 1949, while Supreme Chief Bernard Commanda was paddling his canoe down Nipising River, he was shot in the back, which we strongly suspect was by the Royal Canadian Mounted police, just because the puppet federal government had lost the Court case to the Indians.

After the death or assassination of the Supreme Chief, the following Convention chief Francis Pegagaboue from Parasound (Parry Sound) was appointed Supreme chief and held the post for two years before he pass away with a heart attack in 1952. In September 1953 at a Convention at Ste Anne de Beaupre, I was appointed Supreme Chief of the North American Indian government.

It was then and only then that I began to learn how the government operates and rules the minds of the Indians. First they formed an organization called the North American Indian Brotherhood spearheaded by General Crerar, who at that time was superintendent general for Indians in Ottawa, and Andrew Pauls from Vancouver was named as president and he approach by letter all Indian chiefs in Canada to join his organization and the federal government amended the white man act for the Indians, so called Indian Act; for the first time Indians were used in almost every thing that was done by puppet government, the same rulers who had lost a court case to the Indians.

Ever since that time the federal government has master minded, all the tactics to assimilate the Indians as fast as possible and they are using the Indians to do it for them, and the Indians or the traitors they took all kind of grants under section 68 of the Indian Act. Then the Government gave the Indians the right to vote in federal elections and today it is open for an Indian to vote in provincial election. But you will have to remember if you take that right under section 68 of Indian Act then every day you are closer to be under the provincial government rule and this act was implemented by North American Brotherhood who have taken big part in amending it.

Today lot of these traitors whom called themselves Indian defenders they have voted in every white mans election and taken all kind of grants from the government in every form. They expect to make us believe that they could effectively defend the rights of North American Indians - too stupid to know how they have become citizens to these people they have vote for and taken the grants from.

I know for a fact that some of those traitors have used the grant to pay for the portraits of themselves for $\$ 500.00$ to be hung on their office walls and worse of all these who called themselves Chiefs under the so called Indian Act, and presidents and vice presidents of all organizations whatever you want call them under the direction of 
so called Government of our country and some will question your position just by saying I am the president of such organization and worse of all they don't even stop and think who have master minded their organization. Are they Indian or non Indian (?)

You will have to remember one thing Jean Chretien said: "Anyone that will take benefits from us (are) automatically become Canadian Citizens."

Vine Deloria jr. writes in his book "Custor died for your sins". Individual tribes show incredible differences no single aspect seems to be as important as tribal solidarity, tribes that can handle their reservation conflicts in traditional Indian fashion generally make more progress and have better programs than do tribes that continually make adaptations to the white value system and on page 30 of his book, this would be quite tragic as there is a fundamental difference between Indian and sports fishing. Indian people are fishing for food for their families sportsmen are fishing for relaxation and recreation. Indians my have to starve so that whites can have a good time on the weekends if present trends continue...the again on page 26 of the same book within the NCAI (coalition) personal leadership determines policies and programs in 1954 congress began the great push to abrogate Indian rights in a series of termination bills ... all of this started from the results of North American Indian Nation Court in Quebec city 1949-1950. Then again on page 28, Vine Deloria says in his book, the National Congress of American Indians could cause a great conflict between reservation and non reservation groups in the future. There is little doubt that urban Indians have more sophistication than do reservation people and now urban Indians and the national Indian youth council have formed together as cooperating organizations to work for urban and young Indian people.

It will probably take several years for Indian tribes to absorb the meaning of this new coalition. By then it may be too late for them to survive.

Funding of native associations introduction: the sub committee comprise representatives from Indian affairs and northern development, secretary of state, national health and welfare, privy council and treasury board, the assistance provides in 1970-71 was related to the wish of the government to expand and develop programs for disadvantaged citizens and ethnic minorities. Thus the program of sustaining support to these associations is directly related to the new citizenship objectives.

During 1970-71 a number of new associations were formed and others consolidated or expanded their operations. The result has been the submission of budgets for twenty four native associations requesting funds in 1971-72 totaling $\$ 19,000,000$, nineteen millions dollars of this amount, approximately $\$ 8,000,000$, eight millions has been submitted to the department of secretary of state for basic operational cost (see appendix "B"); in its review the sub-committee took as an underlying principle that the federal government should not be the sole contributor to the support of native associations and the associations should be encouraged to seek funds from other sources. Does this mean taxing the Indians to support the associations or they will be forced to give themselves away to provincial governments and this would mean assimilation. 
Because section 68 of Indian act - its definition is municipality (of) which (is) automatically under provincial rules and I will leave you with this to decide what supreme chief means if one is appointed by Indian mind Indians or one who is elected with poll box same as white man and who is being paid by grants from governments of all levels.

The copies of this letter will go along way since a question was raised from same government paid chiefs and as well to all presidents and vice presidents. I wish you all the luck in the world with your lawyers if you have voted and taken any grants in any form then you will have to give away James bay in order to obtain something.

Your friend

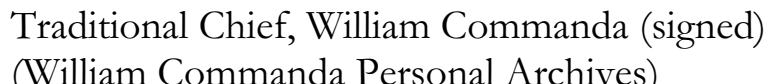

Obviously this letter provides an extremely important political analysis, and first hand account of WC's thinking. Ironically, it can also be applied to an analysis of the continuing malaise in Government/Indigenous politics, despite moments of good will and intent. This and other critical archival material will be examined in detail in future research.

During the sixties, the federal government had become alert to the rising surge of resistance awakening amongst the Indians and developed their own strategy to address this, with the 1969 Assimilation White Paper. Note that between 1951 and 1970, WC was also chief of his community, and accustomed to challenging Indian Affairs regularly around a multiplicity of issues of concern to his people, including with respect to residential schools. Between this and his work with the North American Indian Nations Government, he was acutely aware of the deficits of the White Paper. On September 30, 1967, Indians from across the country arrived to assert their rights at the commencement of the 30 Session of Parliament, and to challenge the White Paper. (This was also the year of WC's first major international gathering, inclusive of Indians from both Canada and the United States). This period was considered the moment of the awakening of the Sleeping Giant amongst Indians; in part, this was a land-based idiom linked with the rock in Lake Superior (Personal 
Conversation Cliff Summers, Oneida, August 2017). It was in 1968 that WC addressed his concerns to the new Prime Minister Trudeau, during the sharing of the plate of beans noted in the famed Trudeau/Commanda photograph (as discussed below), and thus he played a critical role in dealing the real death-blow that came to the 1969 White Paper.

As it happened, the bureaucracy worked with some natives from the west and undermined the intentions of the North American Indigenous Nations Government, in WC's opinion, luring and bribing them into continued serfdom with the creation of the Native Indian Brotherhood, later reconstituted as the Assembly of First Nations. He refused to sign on, being the only one of the six hundred plus chiefs not to do so. Even his own people were angry with him, accusing him of wanting to deprive them of the resources of the federal government by his rejection of the federal plans. But, he could see the dangers ahead with the proposal to replace Indian agents with Indian Chiefs still under the absolute control of the Indian Affairs department. He turned aside, but fifty years later, in 2015, it was quite apparent that things had not improved for the First Peoples of the country at all. In July 2017, the National Chief of the Assembly of First Nations was informing the Special Rapporteur on Indigenous Issues of the United Nations of the still abysmal conditions of the clear majority of First Peoples. In September 2017, the Prime Minister reiterated the same. Perhaps the political intention now is to trigger global reflexivity to heal colonial wounds.

\section{c. History and Environment: 1987 Aboriginal Roundtable, Wampum Law, and Mother Earth}

The Sacred Wampum Belts guided WC's work for forty years. He had become carrier of the Belts in 1970, and brought them out publicly in 1987. The Ojigkwanong documentary draws attention to WC warning about the looming environmental crisis on the national stage; during the last of the historical Constitutional Meetings hosted by Prime Minister 
Mulroney in 1987, WC raised the issues, not so much of Aboriginal rights (note, he had done that with Prime Minister Trudeau 20 years earlier), but about the pollution of waters, earth and air. When the Royal Commission on Aboriginal Peoples released its report at Victoria Island in 1996, he asked the country's leadership whether they would be prepared to drink the waters of the Ottawa River. He was fully mindful of the contamination of the E.B. Eddy and Domtar plants at the Sacred Chaudiere Site. For him, the survival of the Indigenous peoples was inextricably linked with the health of the environment. He hosted two annual Circle of All Nations gatherings in 2002 and 2004, where water conservation was the key theme. In 2006, he hosted the Waterlife Workshop in Ottawa, this one focused primarily on the plight of the Ottawa River. William Commanda was also passionately concerned about the near extinction of the American Eel. He was a key voice in the challenge to see this magical ancient fish of sacred and material importance to his ancestors placed on the Endangered Species List. Elder Commanda blessed the launch of the Species at Risk Act in 2004 and the Roundtable of 2006, and commented on the amendment of the Navigable Waters Act. He noted that

"In the 1600 s the land transformation began with none of the resource management strategies my ancestors had developed and employed over centuries: with the fur trade, logging, dams, hydro, and nuclear energy, the resources of my peoples of the Ottawa River Watershed gave birth to Canada, and they are now dangerously polluted and depleted. I have said before that I believe Mother Earth is a living creature. She has a body and spirit and veins. The rivers are her veins. If they are blocked and contaminated everywhere, cancers and poisons build up; eventually they kill. Dams, motorized vehicles, foreign animals, fertilizers, pesticides and raw sewage attack the lifeblood of Mother Earth. She has to fight back. It is Nature's Law. And what happens to her, happens to us because we are inextricably connected. (William Commanda's Archives)

In the ensuing twenty-five years after 1987, he single-handedly brought the teachings of the wampum belts and the plight of Mother Earth to the attention of literally thousands of people. 


\section{d. Forgiveness: Outreach, Diplomacy and Animating Ginawaydaganuc}

WC attributes his bout with cancer and near-death vision experience with awakening him to the critical importance of forgiveness. Together with the profound spiritual experience came the realization that some negative things are so horrific that one has to find the capacity for forgiveness to be able to set them aside. Not all things can be righted; this is consistent with the Indigenous practice of gifting to heal divisions. This is inclusive of wampum exchange, pipe and other ceremonies, integrated with spirit of generosity, performed and reinforced in community potlatch and giveaway ceremonies. The benefits of forgiveness were the relief from debilitating grief, anger and victimhood. Thereafter, one could tap the wells of personal power. The first step in the journey was to contemplate for what one might need forgiveness. WC's guidance was to contemplate this beside a tree or rock, and then let your pain pass to the Mother Earth for transmutation. Then one would be able to access the capacity to forgive. Many of the inmates in the NFB documentary came to his annual gatherings in the following years, and helped with many of the chores, as our photo files attest. They were on their way to becoming Good Enough for Two (this is the title of his canoe documentary, and it is also a phrase WC used constantly, when asked how he was). Thus forgiveness had two key purposes: to reclaim personal power, and to permit mending of relationships.

\subsubsection{Second Narrative - Indigenous Visibility and Leadership Presence}

Canoe Builder, Respected for what I can do with my hands - here the particular discourse begins in 1981 with the building of a canoe in Roskilde, Denmark, and the meeting with Queen Margarethe; I also examine other factors contributing to a new representation of the Indigenous in the Nation's consciousness, as a force to be acknowledged.

WC and his wife Mary, and his friend Roger Lafleur and his wife, spent six weeks in Denmark, building a canoe for Queen Margrethe. Two books were created in Danish, and 
the canoe rests in the Roskilde Viking Ship Museum. His meeting with the Queen and her two sons, who kneeled to greet the Indian Chief, was a deeply moving experience for him, because he finally felt respected for what he could do with his own hands. She is an artist herself, so likely really understood the importance of the creative energy we discuss elsewhere in this thesis. WC's political astuteness was razor sharp; he asked her how many colonies she had and she replied, "None whatsoever; we gave the last one back, Groenland", to which WC replied, he wished she were his queen! (Thumbadoo/Commanda Unpublished Joint Journal 2007). He referred to the genocide of the Beothuks in the 1987 Constitutional discussions, in the context of the messages of the Seven Fires Prophecy Wampum Belt, and mentioned this in all his readings of the belts. In a sense, the meeting with the Viking queen was completing a circle that commenced with the murder of Beothuk Skraelings when they were first sighted by the Vikings in 1000 , with reconciliation a thousand years later. WC ignited attention to the story the Beothuks. The story continues to develop. Circle of All Nations colleague Derek James created a commemorative Beothuk banner that WC signed. Chief Joe Mi'sel of Conne River Newfoundland (Andersen and Crellin Mi'sel Joe An Aboriginal Chief's Journey 2009) commenced negotiating the repatriation of Beothuk remains from Scotland, and in 2016, the Department of Canadian Heritage engaged in the effort. Efforts are also underway to advance a vision to create the Mount Beothuk Historic Site in Newfoundland.

The Ojigkwanong documentary also presents WC at the inauguration of the Human Rights Tribute with his Holiness the Dalai Lama. WC presents the Sacred Seven Fires Prophecy Wampum Belt, and it is interesting to note that he makes a point of saying, "We have never surrendered". The importance of the occasion was not lost on him, and he reasserted sovereignty at a historical global moment. This was a global human rights initiative launched 
in the capital city of Canada, the city being the ancient meeting place of the Chaudiere Falls. In view of his links with the United Nations from its inception, it was most appropriate that it was he who blessed the event. The messages on the monument are in Indigenous languages and English and French. It was also significant that it was the Carrier of the Sacred Three Figure Welcoming Wampum Belt who welcomed the global spiritual leader. WC pointed out to me that the then Prime Minister did not greet the Dalai Lama, because of Canada's relationship with China. Indian Affairs Minister Flora McDonald was there. It is also interesting to note that the Dalai Lama raises WC's hand to his lips in the video recording of the event. He was obviously recognizing that he was in the presence of someone of spiritual importance. WC met him on three other occasions, and the last, which happened on Parliament Hill, was perhaps most interesting. At this point, something deeper appeared to have resonated for the Dalai Lama, in that he was seeing WC as an Indigenous person with few rights in Canada, while he, the displaced person from Tibet, was becoming an honorary citizen of this country. WC recalled him rubbing his head against WC's in a gesture of acknowledgement of spiritually connected souls.

The documentary notes WC's welcome of President Nelson Mandela at the Human Rights Tribute, on the $50^{\text {th }}$ Anniversary of the United Nations Universal Declaration of the Human Rights. WC was most taken by the president's capacity for forgiveness after 27 years of harsh imprisonment. Both men were also linked to the Wolf Project, a grass roots initiative that honours people who promote racial harmony. WC and President Mandela were presented with the Borealis and Australis Wolf awards respectively. (WC welcomed six of the South African High Commissioners to Canada. It is likely no wonder that he eventually went to South Africa, the motherland. This was to attend the 2001 United Nations 
Conference for the Elimination of Racism and Zenophobia, which was followed, most regrettably for the entire world, by 9/11).

In the context of this discussion of world leaders, I note that it was WC who introduced me to the non-violence philosophy and strategy of the Mahatma Gandhi, and he also welcomed two members of that family to Ottawa. In 2006, we visited an Artist's Exposition in Montreal: Artisans de non-violence. It featured a painting of WC as peace maker. The following is an excerpt from a Circle of All Nations report.

His portrait had been placed beside one of Henry David Thoreau. What an absolute irony and what a full circle, I thought. Now most of you may know that Henry David Thoreau, of Walden Pond fame, influenced many with his philosophy and ideology evolved of a reverence and respect for nature; this included Tolstoy, who in turn influenced Mahatma Gandhi, who influenced Martin Luther King, who influenced Nelson Mandela, and of course many others we think about as the wise peace activists of global importance.

But how many of us know that Thoreau's wisdom emerged from the teachings of his Penobscot guides, William's Algonquin ancestors, the ones who taught the lessons of Nature. (Excerpt from Circle of All Nations 2006 Mid Year Report).

(This is afffirmed by the closing line in the following text "We do not learn by inference and deduction, and the application of mathematics to philosophy, but by direct ... sympathy. ... The most scientific will still be the healthiest and friendliest man, and posess a more perfect Indian wisdom" (Thoreau 1906 131)

\subsubsection{Third Narrative - A William Commanda Lament}

Noble Savage - the new age revival of the image of the noble savage, produced in the vein of "othering" (Said 1978), a romanticized overview delimiting the discourse.

In this narrative, I examine the continued obliteration of the critical voice and views of Indigenous leadership such as WC had articulated and animated for sixty-five years, though by the eighties and nineties, Canada was beginning to acknowledge him. Perhaps WC orchestrated that himself. In 2017, I met George McDonald, the first President and CEO of the Canadian Museum of Civilization, who, following west coast tradition, invited WC to offer a welcome for the Bill Reid visit to Ottawa. WC took on the responsibility with aplomb 
and presented the Haida artist with a miniature birch bark canoe; Bill Reid then gave WC one of his paddles that had been commissioned for the Museum. Thus, WC launched visible Algonquin presence into the capital city in a demonstration of agency discernable in his unique discourse. He was very well received everywhere, and he winked when he said to me, they think I am a Noble Savage!

But actually, WC was demanding nobility of Canada. In the document, Circle of All Nations Reflection on George Grant's Lament for a Nation, as viewed through the lens of William Commanda, (www.asinabka.com), we make the following observations: "There has to be a great noble change" (William Commanda), that "Posterity may know we have not loosely through silence permitted things to pass away as in a dream" (George Grant 199526 second publication, Carleton University). WC was grappling with the same political, social, neo-capital and environmental challenges as Grant, but from his position of groundedness on motherland.

Written first in 1963, the Lament was published in 1970, and by this time, following the Vietnam War, Grant notes "Even the Canadian bourgeoisie can see the perhaps unresolvable racial conflict, the expansion and decay of its cities, the increase of military influence in constitutional life, the breakdown between the generations, the effects of a century of environmental spoliation etc. etc. (that is, the dangers of the domination of the American way, stalking the public world)" in the existent ontological package (Grant 1995 9).

The Lament is timeless in many respects, even today instructive in its analysis of the political power brokering, and striking in its anticipation of the developments of the past decades, and the present moment. Here, we are no longer feeling the impact of continentalism alone - this is the age of globalization; and capitalist domination, neoliberalism, racial conflict, military control and environmental despoilment have 
accelerated with little promise of attainment of the grand dream, or any clear insight into the morass.

In fact, that ontological package has "concentrated incomes and wealth among a few, squeezed the middle income strata, and fueled unparalleled inequalities in income, wealth, and life chances. If there is one consistent indicator of neoliberal governance, it is stalled, if not declining human development and well-being amidst unprecedented economic growth and wealth creation. It is a governing formula that is ripe with all manner of social injustices." So says researcher Janine Brodie, on Reforming Social Justice in Neoliberal Times (2007). Canada had just dropped out of the UN's 10 most developed countries list on its human development index. On the global scale, the realities are even more lamentable.

George Grant's Lament is a political meditation, and it reaches beyond this nation and the defeat of Canadian nationalism, and his times. His question, "Were there phenomena in our own heritage which could sustain some resistance and provide some moral ballast to the apparent soulless world order forming around us?" (Grant 1995 18) is relevant here and now, and the answer William Commanda offered spans time and space. Grant notes that "The Canadian economy was part of the total resources of North America; Canada was an undeveloped frontier within that total, and the capital necessary for that development would come largely from the United States; that North America was committed to a capitalist structure in which the control of production would be in the hands of "private" corporations, while the government would only play a supervisory role. In his Lament, Grant ponders, "If there had been an influential group that seriously desired the continuance of the country after 1940, it would have needed the animation of some political creed that differed from the capitalist liberalism of the United States. Only then could they have acted with sufficient decision to build an alternative nation on this continent... But no 
such tradition existed among any of the important decision-makers in Canada. The only Canadians who had a profoundly different tradition from capitalist liberalism were the French Canadians” (Grant 1995 60).

Grant was not entirely correct. He did not appreciate the position of the Indigenous Peoples in Canada. The Indigenous were invisible in the Canadian nation building politics of the forties and the sixties. However, that does not mean that they were inactive. Despite the devastation of colonization, decimation by war, disease, poverty and spiritual assault, despite being torn apart by land division and expropriation, and genocidal residential school abuses, the Indigenous peoples rallied repeatedly to challenge changes in their homeland.

Over the past century, the Indigenous voice persistently demanded political presence and equality at the global stage. WC, an avid student and historian, knew that in 1492 Pope Alexander VI and European kings had laid the foundation for the colonization and extermination of non-European peoples. He knew the North American Indigenous population fell from an estimated 72 million to 4 million in a few centuries. He also knew that in 1532, Franciscus Di Vittoria, founder of modern international law, had affirmed that the Indians were the true owners of the land, and that the "pearls of the seas" belonged to them; this is where "Aboriginal Right" takes its roots in North American. Four hundred years ago, Samuel de Champlain met his Algonquin ancestor of the Ottawa River Watershed, Tessouat, acknowledged leader in the American North East, and they struck the first recorded alliance between the newcomers and the people of Turtle Island, as the continent was called, to guide travel and exchange of resources. By the 1700s, his ancestors had created the sacred Three Figure Sharing Wampum Belt, agreeing to share values, land and grand natural resources in three equal parts with the newcomers, then the French and English; this sovereignty principle was later affirmed in the Royal Proclamation of 1763. (It is interesting 
to note how history and facts are constrained to serve the dominant will; in historical texts, Tessaout's name and appearance as the one eyed sagamore and his presence in both Tadoussac on the St. Lawrence and in the Ottawa River Watershed is left unclear, undermining his territorial reach; around 2009, the horse and buggy tour guide in Old Quebec City informed us that someone with somber appearance but also found guilty of fraud had posed for the Champlain portraits.)

WC understood that Europe, first after the 1500s, and later after the first and second world wars, emerged from darkness through the exploitation of the grand natural resources, the gold, furs, and wood and minerals, of the Americas. He was also aware of the hanging of Louis Riel, the Metis leader who created the first provisional Indigenous government in Manitoba. In fact, he performed a pipe ceremony at his grave. He was fully aware of the exclusion of the Indigenous voice from the public debate about the Canadian nation.

In his book, What is a State, Bob Jessop offered the following interesting observation. Isaiah Berlin, in his study of Tolstoy, compared the hedgehog, who knew "one big thing" to the fox, who knew many things (Jessop 2002 45). The scientific, rational orientation fixing homogeneity and sameness, and universalizing, in ever-expansive ways, he found, was stripping nature and people of creativity, and standardizing sterility.

The Indigenous effort to impact the definition of nation was brave, passionate and persistent. It was also inclusive of a fervent desire to protect the land from ceaseless exploitation. Already in the 1940s, the North American Indigenous voice was raising the concern about environmental degradation and climate change, submitting a petition to this effect to the United Nations. Under the leadership of the Hopi, the Indigenous voices drew the attention of scientist, Albert Einstein, and Aldo Leopold, the American father of environmentalism also noted the shadow Indian. In poststructural idiom, WC and 
Indigenous peoples around the world from the time of the creation of the United Nations, and even earlier, had been challenging the cultural construction of nature. The 1990, publication of The Gaia Atlas of First Peoples, written by Julian Burger with campaigning groups and native peoples worldwide, was followed by the 1993 first United Nations conference of the World's Indigenous Peoples, Cry of the Earth, where WC was the first to deliver his message. WC's own uncle Gabriel Commanda penned letters to the Canadian government about the decimation of wildlife and devastation of habitat throughout the Ottawa River Watershed in the 1920s as a result of the environmental abuses of the hunters, trappers and tree cutters. He, Anahareo Gertrude Bernard and Ignace Papatie, chief of Grand Lac Victoria band, Algonquins, were the first official environmentalists of the watershed (Ferguson L'Algonquin Gabriel Commandant, Biographie romance d'un pionnier de l'Abitibi 2003, www.mattawamuseum.com/english/anahareo, History Museum, Mattawa, Ontario). Anahareo Gertrude Bernard, wife of Grey Owl, was one of the earliest internationally acknowledged conservationists. Her work as naturalist and conservationist was first recognized at an international level in 1979 when she was admitted to the Order of Nature by an animal rights based organization in Paris. Eventually, Canada was obliged to acknowledge her and the Governor General flew to her bedside to present her with the Order of Canada in 1983. In 2006, on the $100^{\text {th }}$ year of her birth, a museum was opened in Mattawa, Ontario and it honoured her work and writings. WC offered the blessing. She and Uncle Gabriel Commanda were profiled at the 2006 Circle of All Nations Water Life Workshop. But they were shadowy ghosts in the limelight of the likes of Grey Owl. As primitives, their voices were annihilated or subsumed. I have also seen in WC's archives a document articulating environmental concerns, created in Hawaii, and presented to the United Nations General Assembly in the mid-forties by Hopi elder Thomas Banayaca, on behalf of several 
Indigenous tribes. In 1993, consistent with their prophecies that had told the Hopi they were to knock on the door of the House of Mica, the United Nations building, four times, he delivered their last message to the UN, a major storm and flood erupted, flooded the UN building, and marked the first UN Indigenous conference, the Cry of the Earth, and the launch of the UN Decade for the Culture of Indigenous Peoples. William Commanda was there, the first to deliver his Indigenous remarks and the wampum belt messages (Video recordings, Wittenberg Centre, Woodstock, New York 1993). A French documentary on The Kogi, Message from the Last Man (by Eric Julien 2006), of barefooted, isolated shaman from the mountain caves of Columbia, reveals an uncanny, apocalyptic, contemporary articulation of the global human and environmental crisis. Individually and collectively, Indigenous voices have challenged the materialization of nature and her reconstitution as commodity.

In his animation of his heritage, grounded in the earth-emergent biodiversity, WC projected complexities, inter-connection, contextuality, mutuality, stimulation, evolution and open-endedness. This is the Indigenous current of the Wampum heritage and a circle of all nations that he sparked in countless people of all social, cultural and economic and educational backgrounds.

WC saw further and much earlier than most, into a future of unceasing capitalism, exploitation, corruption, environmental degradation, species destruction, resource depletion, climate change and war. He also saw a collective mental and moral demise looming. WC saw, as George Grant did not, that the Indigenous are the glue to restore a broken world, and he blessed the seeding of this work at the Sacred Chaudiere Site, the sacred meeting place of his own direct ancestors. Countless Indigenous and non-Indigenous peoples lit that fire with him; rather than a mere lament, he offered a prayer and challenge that all come to understand the urgency of call, before the end of time (William Commanda Constitutional Debates 1987). 
This is the "thrust of intention into the future" that Grant sought so fervently. Indeed, even Grant's reference to Milton's Abdiel, “Unshaken, unseduced, unterrified” prefigures the stature of William Commanda (Grant 1995 60).

WC exhorted us tirelessly to "Come together, with one heart and one mind, with one love and one determination to create $A$ Circle of All Nations, A Culture of Peace", and ignite a legacy of hope for the future, not just for the nation, but for the world. In his tireless work, being far more than a noble savage, WC called repeatedly for a great noble change in all of us.

\subsubsection{Fourth Narrative - A Sampling of William Commanda's Teaching Tools}

Virtuoso Performer - William Commanda/Circle of All Nations discourse: the fourth discourse presents his animation of his own life and work, as one grounded in his relationships with his Mother Earth, and with the earth-based ceremonial, spiritual, heritage, traditions and knowledge acquisition and knowledge generation practices informing and developing this relationship.

\section{A Circle of All Nations Medicine Wheel Epistemology}

William Commanda animated his Circle of All Nations, a global eco-community, as a bridge developed around the concept Ginawaydaganuc - "We are all inter-connected", to awaken people to the Law of Nature principle grounded in the conceptual framework of relationship, animating syntropy, by stimulating negentropic shift at multiple individual, communal and advocacy levels. This thesis itself is designed and organized, in pentimento fashion, to dissect and illustrate some of the serious reflection that is incorporated into the utilization of the model, and demonstrate the potential for pedagogical application.

He animated his relationship-generating Circle of All Nations work in multiple ways. Over the past two decades, this has been organized graphically under the formal identity of a Circle of All Nations; this is, at the same time, an unfunded informal global eco-peace community. WC organized many activities each year at his home in Kitigan Zibi, Quebec, on, Victoria Island, his ancestral homeland, and in the National Capital Region and vicinity. 
These focused on sustainable relations, environmental stewardship, peace building and healing. He also attended local, government and international events to advance his reconciliation and educational work. The absence of a formal funding structure has no doubt impacted the potential of his reach. Yet despite this and multiple other challenges, his work still appears to engage the on-going interest of a diversity of people, including the academy. The overall work has been documented extensively in his websites, books, and several videos; information on his priorities is input regularly into several Facebook pages (www.circleofallnations.ca and www.asinabka.com).

The following section is illustrative of few of the pedagogical tools that complemented WC's textuality, semantics and semiotics. The combination of technologies served in renegotiation of boundaries with a diversity of peoples, and, beyond language, served to bridge understanding and build relationships. Elements are dispersed in a consistent, discursive web that shape shifts in alignment with individual and communal propensities, but does not lose its integrity, rather like the Spider's dreamcatcher web. They inculcate cyclical and spiraling approaches to planning, action, development and knowledge generation grounded in earth inspired motionality, reinforced on multiple planes with a diversity of people.

\section{Circle of All Nations Planning Medicine Wheel Tools}

In the late 1990s, WC built a teaching lodge at his home; immediately it was ready, he went off to purchase an overhead projector to deliver presentations. He demonstrated the attentive, emergent agency of an avid teacher, and he embraced information and pedagogical technology with tremendous enthusiasm. Twenty years ago, we developed the Circle of All Nations medicine wheel model as a conceptual tool to guide the work and teachings.

The following section includes discussion of the medicine wheel conceptual tool from our 
book, Learning from a Kindergarten Dropout (Thumbadoo 2005); this is followed by the presentation of several key examples of the model, as adapted for various Circle of All Nations projects over the years. We used it to support our project planning and development efforts; we found the basic model a great help for conceptualizing and developing projects; it also taught patience as the efforts evolve within the context of the ongoing spiral of life. The discussion and images are included to demonstrate how the associated Medicine Wheel image was utilized and adapted to serve a wide range of environmental education objectives.

The model has been shared widely to inculcate cyclical and spiraling approaches to planning, action, development and knowledge generation grounded in earth inspired motionality, reinforced on multiple planes with a diversity of people. It is documented in our books, has been available on three websites since 2000, and on Facebook since 2009. The Circle of All Nations Frog Principle Model (for advancing reconciliation) was originally developed on transparency at least seventeen years ago; the word cloud images now generated by computer are an evolution of that idea! Likewise, in 1998, we arranged for Hopi elder Martin Gashwaseoma from Arizona to engage in discussions with WC over the airwaves and television as a special workshop during the Circle of All Nations gathering of international participants; both were old Indigenous prophecy holders, and they engaged in a Skype type webinar, before Skype existed! In an unpublished paper entitled Indigenous Environmental Ethics we wrote the following about a conceptual medicine wheel framework:

Grounded entirely in the World of Nature, the Medicine Wheel is an ancient symbol used by the Indigenous Peoples of North and South America to intimate the complex nature of physical and metaphysical life, and in its inexhaustible layers of meaning holds truths pertaining to the supremely complex, biologically diverse, ever evolving, living planet, Mother Earth; local, global and universal environments; the planetary and stellar worlds; principles of evolution and life; of space and differentiated space; time, time markers and eternity; cardinal and metaphoric directions; the key elements - fire, earth, water and air; animate and inanimate life, plant, animal and human life; dimensions of human nature, growth, development and potential; concepts for sustainable living; symbols of inspiration, vision, 
guidance, instruction and wisdom, and indeed, much, much more. Understood and articulated differently by different Indigenous Peoples, or not articulated at all, it nonetheless constitutes a body of understanding held in common, which still informs Indigenous culture in deep and profound ways, and which compels intense study, and holds tremendous potential for expansive understandings of the nature of life. It is incredibly remarkable in that it maintains a coherent core while at the same time it embraces countless diverse forms of expression.

Its precepts constitute the underlying backdrop of William Commanda's thought, action, work, outreach and manner of being, though he does not necessarily always articulate his reality in any concretized fashion. When one explores the incredible complexity and patterns of his life and heritage, and the interweaving of the Medicine Wheel symbol throughout, one realizes what a deep and inclusive metaphor it is for the articulation of his understanding of an Indigenous Environmental Ethics.

The Medicine Wheel spins, underlining the notion of movement, momentum, spiraling, volition and eternity. It is divided into the four cardinal directions, which are not so much point as zones, and each of them circles the central life force, emanating from Mother Earth, going from east, to south, to west, to north. Each of the directions is associated with prescriptive animals, and each of the key elements, fire, earth, water and air, and in turn the elements are associated with messages, ideas and concepts; these serve to guide and inform action, behaviour, and spiritual, physical, emotional and mental growth and development. While not all versions of the medicine wheel will reflect the same patterns, the fundamental symbols are there in all.

When applied directly to a study of the life and work of William Commanda, the following ideas and particularities predominate; and here one observes the grounding principles and values of his ancestors and heritage that have evolved to create the foundation for a way of life, Ginawaydaganuc, which still is the only inclusive message that points to a sustainable future for all - We are all connected/All are related.

Vision Portals to Peace (metaphysical)

Sacred Sites

Petroglyphs/pictographs

Wampum Belts

Sacred Pipe

Ideology

Mythology

People

All My Relations (attitudes, values)

Generosity

Responsibility

Respect

Reciprocity

Balance

Reconciliation

Integrity

Honour 


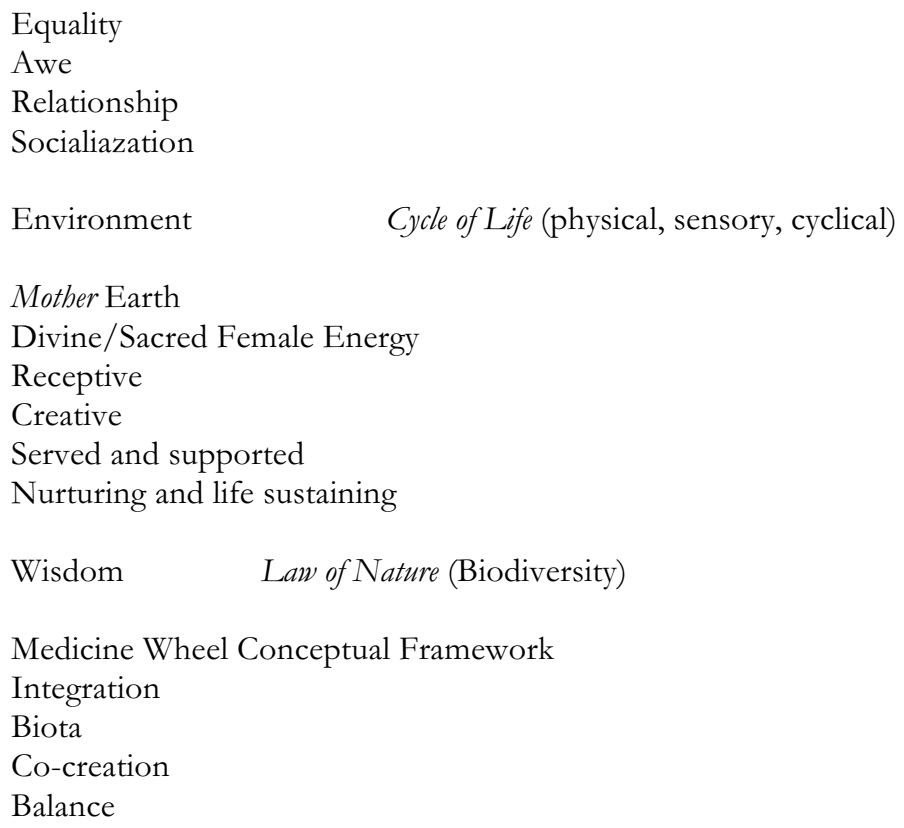

(Thumbadoo/Commanda Unpublished Joint Journal 2007)

The following Circle of All Nations Pedagogical Tools are included to demonstrate elements in his discourse:

1. a. Circle of All Nation Conceptual Medicine Wheel and

b. Examples of Circle of All Nations use of the Conceptual Medicine Wheel

2. Grandfather Commanda's Gatherings

3. FROG CAN Pedagogical Tool

4. A Medicine Wheel Was to Understand Ideological Differences 


\section{a. Circle of All Nation Conceptual Medicine Wheel}

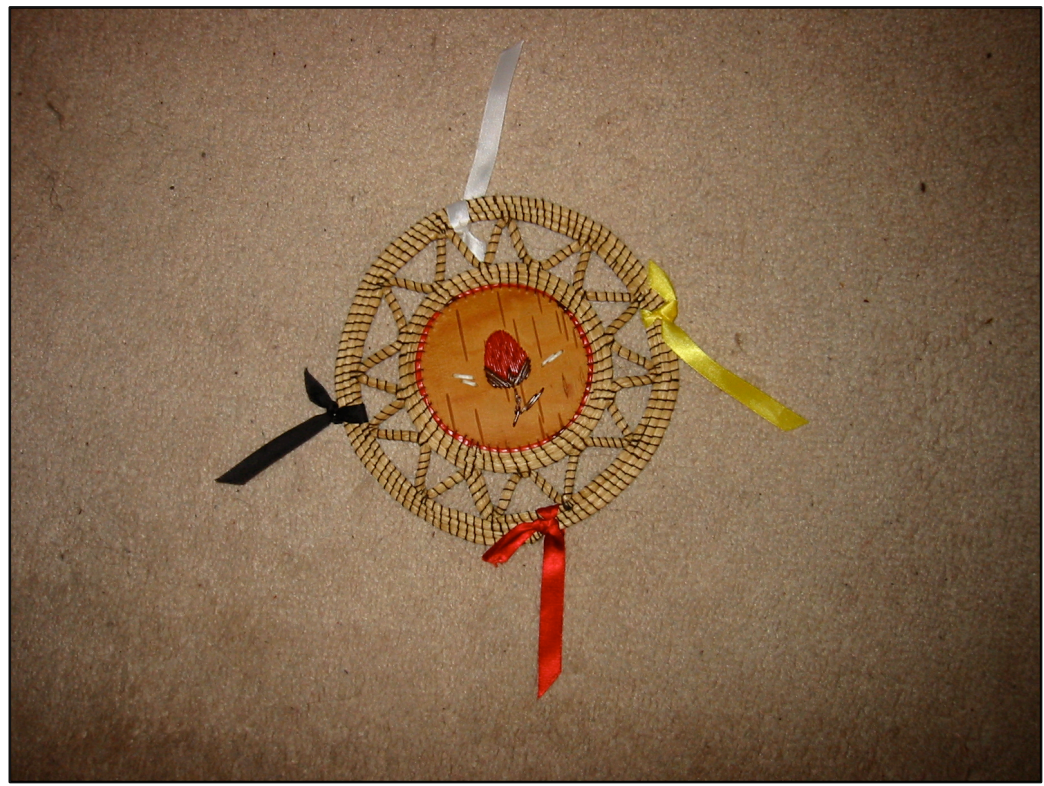

Figure 6. Circle of All Nations Conceptual Medicine Wheel (1a)

William sometimes spins a Strawberry Medicine Wheel during his workshops. The centre is a piece of birch bark and it is bound by weavings of sweet grass. The birch bark reminds one immediately of the canoe, the quintessential reflection of the spirit of the nomad who lives lightly on the earth; this is the heritage of his peoples.

The sweet grass is one of the four herbs sacred to the native people. Its gentle smell is released when sweet grass braids are burned to create smoke for purification, or when it is soaked in water; the other three sacred herbs are tobacco, which carries one's prayers to the creator; sage, which William uses in his smudge bowl for cleansing and placing a circle of protection around a person; and cedar, the powerful herb for healing.

The medicine wheel has a strawberry, the heart berry, embroidered in the centre one the birch bark in coloured porcupine quills, reminding us while we must always strive for balance between the emotional, physical, spiritual and mental aspects of our nature, we must ultimately be guided by our hearts above all.

The medicine wheel also has ribbons in the four sacred colours, yellow, red, black and white, to reflect the four directions, the east, south, west and north and the accompanying teachings, and the four symbolic races.

We organize our working medicine wheel following the flow of the four sacred directions, and focus on the four sacred directions, and focus on four core areas, vision, community, healing and wisdom in each section. 


\section{We associate the Eastern Direction with Vision}

We associate the image of the morning star and the sacred wampum belts with this section, as they underline the thread linking the work to the inspiration of the Elder's ancestors; this reflects both the very personal nature of this aspect of our medicine wheel, as well as the linking of vision with spirit.

The vision section of our medicine wheel focuses on the seed, vision and planning activities that inspire the different aspects of our work and lives.

We connect with the cocooning energy of insects and the crawling creatures with this generative period.

By and large, we organize our visions and plans and opportunities for renewal here.

It is true that we collapse many images and symbols into this section, (and likewise in the others) which might also serve as well elsewhere, (for example, an insect cycle might another time take us around the whole medicine wheel, from the pupa (seed) to larva (growth, incubation) to 'cocooning' work (development) to the flying insect (maturation). We allow ourselves the flexibility to also see their guidance elsewhere.

We see the energy of Fire belonging in this zone, igniting a passion for new ideas, new projects and initiatives.

When we turn to the Southern Direction, we focus more on people, community and physical life

The squirrel is the symbol we associate with this page. William belongs to the squirrel clan, the clan of the tireless seed planters; they are amongst the most gentle and friendly of the wild creatures; at the same time, as they climb up and down trees headfast, never retreating, and so they inspire courage and perseverance.

They are full of determination, William tells us. (This is) Much like himself - no wonder that his favourite song is Johnny Cash singing, I won't back down!

These, and other four legged creatures also remind us about the importance of strength at the physical level, and of the gifts the six senses bring to us, and they exhort us to maximize our physical potential.

This is the zone where we direct our attention to people to animate and actualize our key activities, gatherings, workshops and training.

We think about those whom we wish to serve, or about those who need to be engaged in an activity in order to advance it.

This is the direction guided by the energy of Mother Earth who with her helpers teaches her children to live their physical best.

In the Western Direction, we move to bealing and development 
The canoe is our symbol for this portion of the medicine wheel, partly because it figures so intimately and extensive in William's own life, in the nomadic history of his ancestors, and in the psyche of this nation; it supports the journeying within.

Primarily, we see this as the zone for reflection, analysis, and refinement of our ideas and projects. It is the place of introspection.

Here, too, we explore ideas, activities and programs that can contribute to healing at emotional and spiritual levels as well as ideas to inspire personal growth and spiritual development.

This is the zone from which we contemplate the journey of life.

Here too we remember the teachings of the sea creatures; they remind us of the realities that exist at the level of our sub-conscious.

It finds its purpose in Water, which is the element whose influence we acknowledge here.

\section{The Northern Direction is the place of wisdom}

Our symbolic teacher here is the Thunderbird. This mythical creature is William's special helper. Pakinawatik, Thunderstick, Stick Hit by Lightning, William's great, great, grandfather carries some of its energy.

This bird, revered in the legends of the people of North America, much like the famed Phoenix, rises out of its own ashes. It is the symbol of eternal hope, and the seven diamond Thunderbird in William's website inspires the prayer for the lighting of the Eight Fire of the Seven Fires Prophecy.

Birds link the material earth world with the world beyond, the world of spirit, the cosmic consciousness, the other dimension that impacts our lives unceasingly.

This is where we synthesize our best ideas, and look for ways of sharing them.

Our focus in the zone of wisdom is to strive towards an ever-deepening integration of the messages and strengths of all directions in our individual and communal lives.

The Thunderbird draws us to the winged creatures, the bird, and they connect us with the fourth element, Wind. (Thumbadoo 2005 103-105) 
The graphic images that follow demonstrate the use of the model to address a variety of objectives. In a way, this is comparable to the performance mapping reflexivity and repeated reworking of images for knowledge generation by the petroglyph and pictograph engravers.
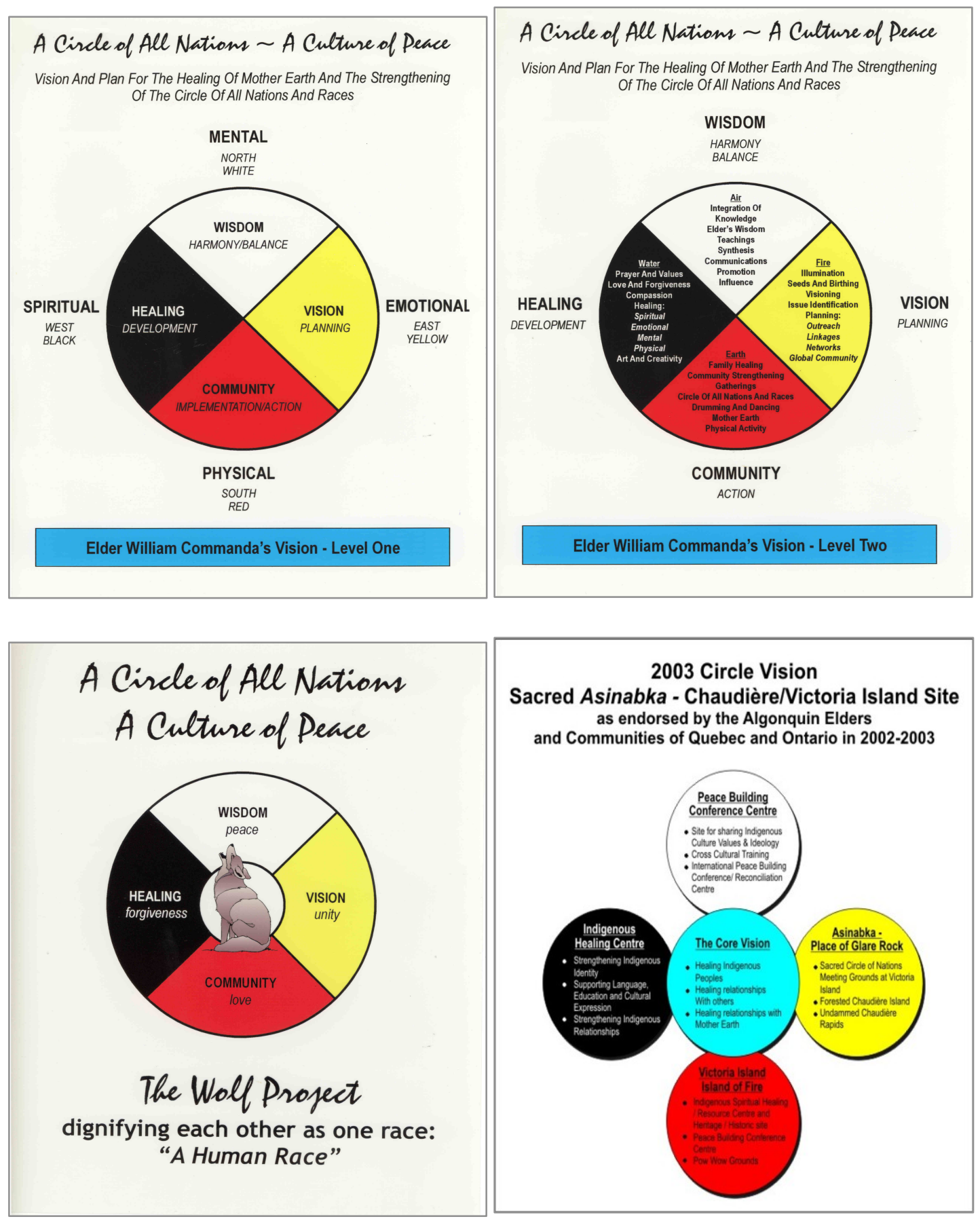

Figure 7. Examples of Circle of All Nations use of the Conceptual Medicine Wheel (1b) 


\section{Grandfather Commanda's Circle of All Nations Gatherings}

The annual gathering he hosted at his home on Bitobi Lake in Kitigan Zibi, Quebec. The extremely popular, extraordinary, land-based example of WC's teaching in action In its second phase of activation from 1996 to 2011, literally thousands of people of all backgrounds and from all over the world made the annual pilgrimage to his lake side home, camped out and engaged in personal and communal spiritual development, healing and experiential learning. The following is a description of the activity from the Circle of All Nations brochure from 2000:

Grandfather William Commanda witnessed the oppression and near destruction of his land and his peoples, language and culture at almost every turn throughout his life. In 1961, diagnosed with terminal cancer, and at a most painful low point of his life, he experienced a profound spiritual awakening, an awakening that placed him firmly on the Red Road of forgiveness, love, compassion and reconciliation.

He saw an urgency in the need to bring together the people of the four symbolic races of humankind, to evolve relationships based on these values, and to create a synergy to contribute to the spiritual transformation of a world seen by many as morally and spiritually bankrupt.

Over the decades, he promoted his vision nationally and internationally in a singularly independent fashion, with no formal infrastructure. People across the world have been touched by his extensive and tireless work and outreach to promote racial harmony and cross-cultural understanding, to encourage and support spiritual development and to raise awareness of environmental issues and the abuse of Mother Earth.

The most special of his activities is the annual spiritual gathering that he hosts at his home every summer. Hundreds of people from far reaches of the globe journey to Maniwaki. People attend sacred sunrise, pipe and sweat lodge ceremonies; engage in discussions on four key themes of great importance to him: Indigenous wisdom and respect for Mother Earth, racial harmony, social justice and peace; and they participate in art, drumming, healing and social activities and workshops. His gatherings are carefully designed to promote individual and group healing, build community, support youth empowerment and development and integrate the creative principle in spiritual, social and community development.

Ultimately, it is Grandfather's deep humility, great love and abiding compassion for all that encourages so many people to connect with their own individual potential, and to spiral into achieving their personal destinies, and taking their rightful places within their communities. (Excerpt from Circle of All Nations brochure Thumbadoo 2000). 
The following is an excerpt from Learning from a Kindergarten Dropout, discussing a Circle of Nations trajectory to developing communities of peace. It is followed by transparencies, which, when laid one upon the other, demonstrate the temporal dimensions of challenges to social change.

3. Frog CAN Pedagogical Tool

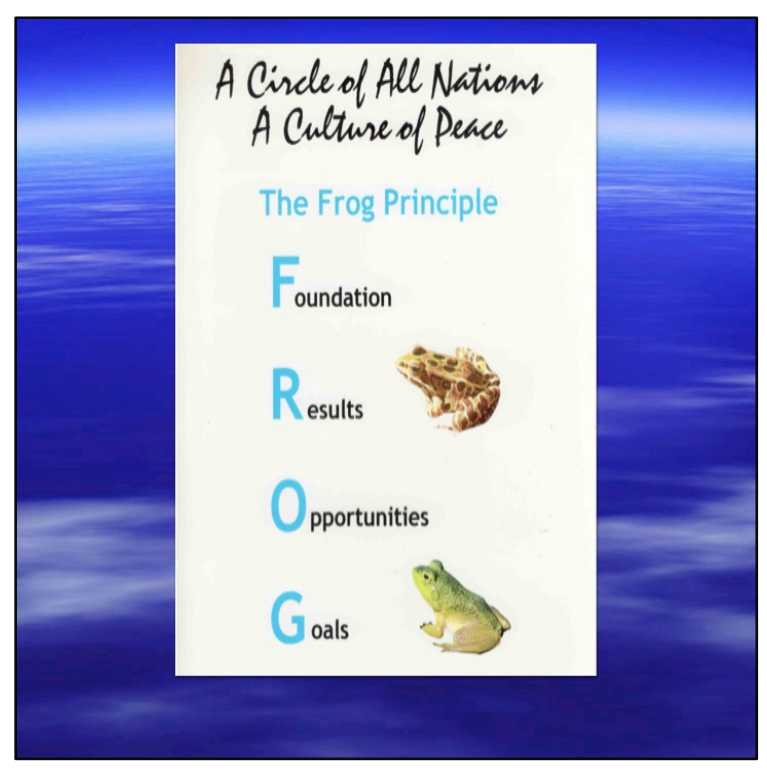

Figure 8. The Frog CAN Pedagogical Tool

Of course, William is pragmatic and practical. His capacity for integrating many ideas and activities can be overwhelming, and his expectations that we can all find our rightful places in this circle of all nations, this culture of peace, can be a little daunting. After listening to him talk about love and forgiveness enough times, I found a way to explain how we can work individually and in groups to become a Circle of All Nations, A Culture of Peace.

I was inspired by a memory of an encyclopedia from my childhood. It contained translucent pages which each in turn revealed more about a frog's anatomy. Thus on the first page, you was the exterior of the frog's body, then on the next, the muscular system, then the vascular and finally the skeletal system. The complexity of the frog's body was revealed there within the layered pages.

So we developed our FROG transparencies to teach how we could become $A$ Circle of All Nations, A Culture of Peace. They move us from exploring Foundatons, Results, Opportunities and Goals in understaning our reality. (Here is the FROG Spirit in work!) Many healers see frog energy as the energy of cleansing and and healing; it is connected with the element of water; it supports the elimination of negativity and restoration of harmony; it is this energy that underlies this effort in our work).

The transparencies do four things:

-they reflect the complexities of our multifaceted lives, histories and heritages, both individual and communal; 
- they show how we can see ourselves reflected in each of the levels of development;

- they provoke serious self-reflection and

- they support deliberated movement towards actuating our better selves and evolving better communities.

As you place one transparencie over the other, you begin to realize how they coexist and contribute to the complexities, frustrations but also hope in our lives.

\section{Foundation}

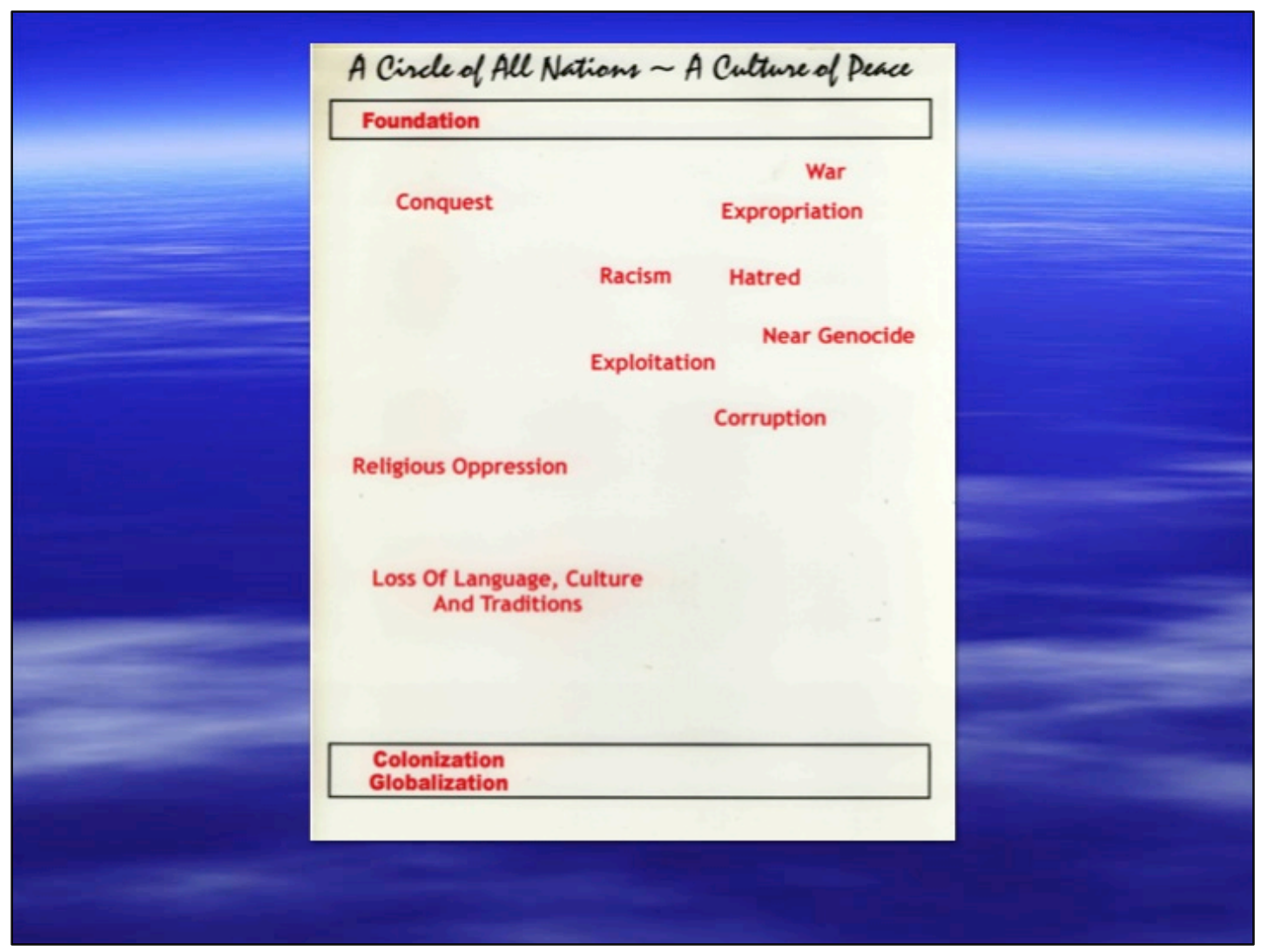

Figure 9. The Foundations Transparency

First we focus our attention on the past five hundred years following the arrival of the newcomers, and see at this level the foundation upon which the history of this land is built. But as we examine these issues, we begin to realize that they are not confined to the past or to governments or groups; we see that the negative legacy of colonization is replicated in many of the ramifications of globalization; and we see where we might be implicated in abuses today too, as individuals and communities. Thus here, we might consider expropriation of land, or an unceasing use of plastic bags with our grocery shopping. William has talked about both these issues ober the years. With respect to the latter, just outside the national capital, they have discovered a fire smouldering in a dumpsite and polluting the air to such an extent that people now have to be evacuated. 
Our explorations in this area teach us bumility. We begin to realize the enemy is not just outside or in the distant past.

\section{Results}

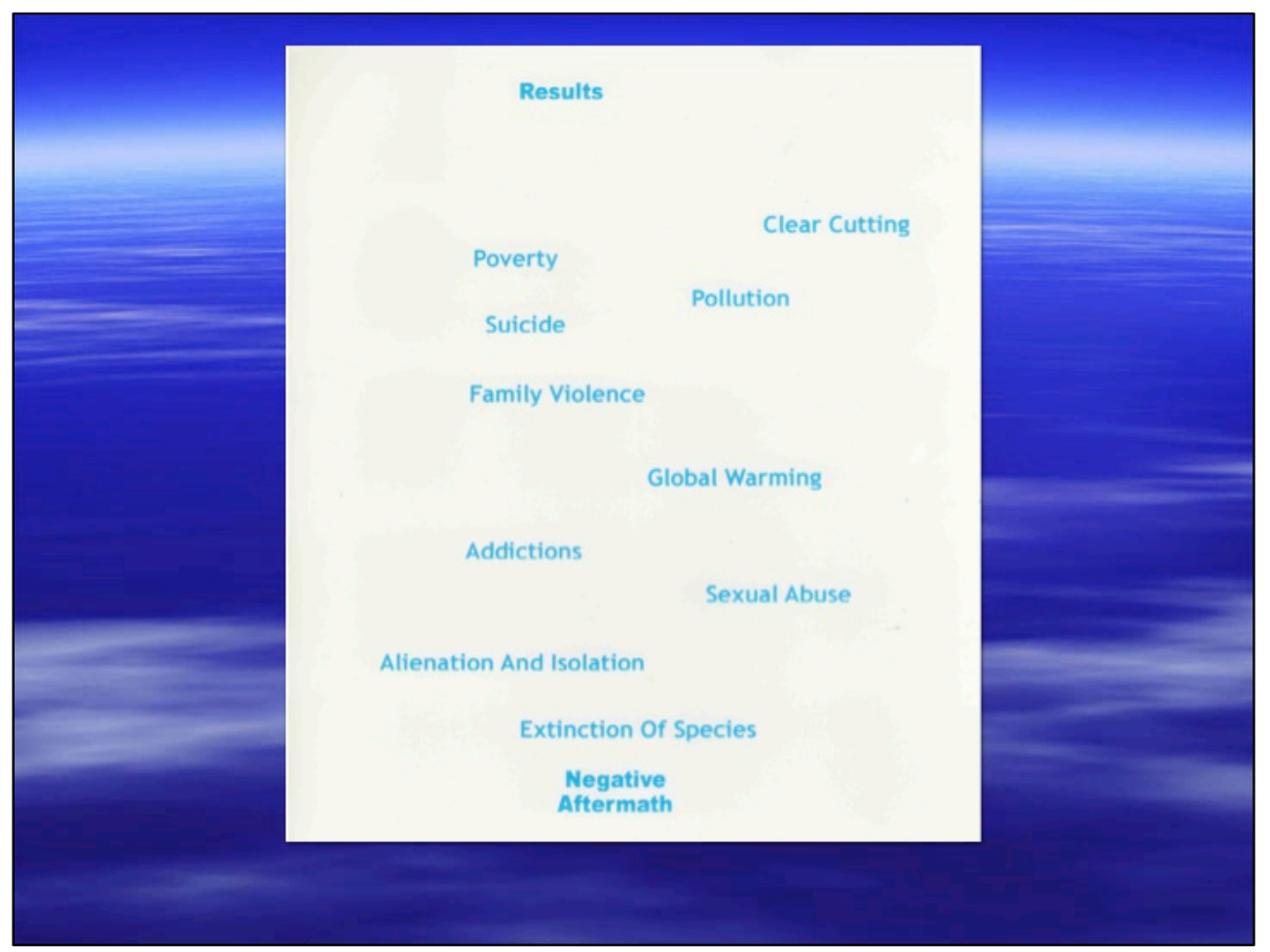

Figure 10: The Results Transparency

Next, we consider the consequencies of the actions of the first layer, for example pollution or war; we also begin to realize how both levels can co-exist. Thirty-five years ago, when I first arrived in Canada, we used to be advised of the count of the pollution index with each weather broadcast. When it reached 22, Stelco and Dofasco had to shut down steel operations in Hamilton. This has long disappeared from our daily consciousness. But the change is deeply ominious. This summer, William and I were in Guelph and we heard on the news that kindergarten children could not go out into the school grounds at lunchtime because of the air quality.

And what about racism, both overt and systemic? Most of you who have attended William's annual gatherings in Maniwaki have met Donald Marshall Junior and have looked at him with some awe; just imagin a seventeen year old tossed into a punitive criminal justice system, living day after day for eleven years, each moment knowing the evil and injustice human beings are capable of, individually and collectively. How does one emerge from that type of experience? And what about suicide? What must it be like really to see life to be so painful as to only want total oblivion? What must run through the minds of the young people of Davis Inlet, and what must their parents, siblings, grandparents, elders and leaders feel? What self-righteous cries we hear about misspent dollars. Surely one cannot expect people to live the first level of reality without ramifications on the next. 
Of course, here, as with each of the layers, we identify only with a small spectrum of the issues that could be examined, and even then, our transparencies are too busy!) One could undoubtedly add many things to the lists, and one should.

As we contemplate the issues exposed deeply, we learn empathy and compassion.

The real point is to contemplate the issues - to sit with them for a while and try to understand them deeply. We might want to go back and take another look at the first layer, the precipitating factors. We may need to move a little closer to really understanding their implications. We need to shake ourselves out of the anethetisized zones our minds have become accustomed to occupying and become truly mindful. That is the challenge. But, William reminds us, we must not despair.

\section{Opportunities}

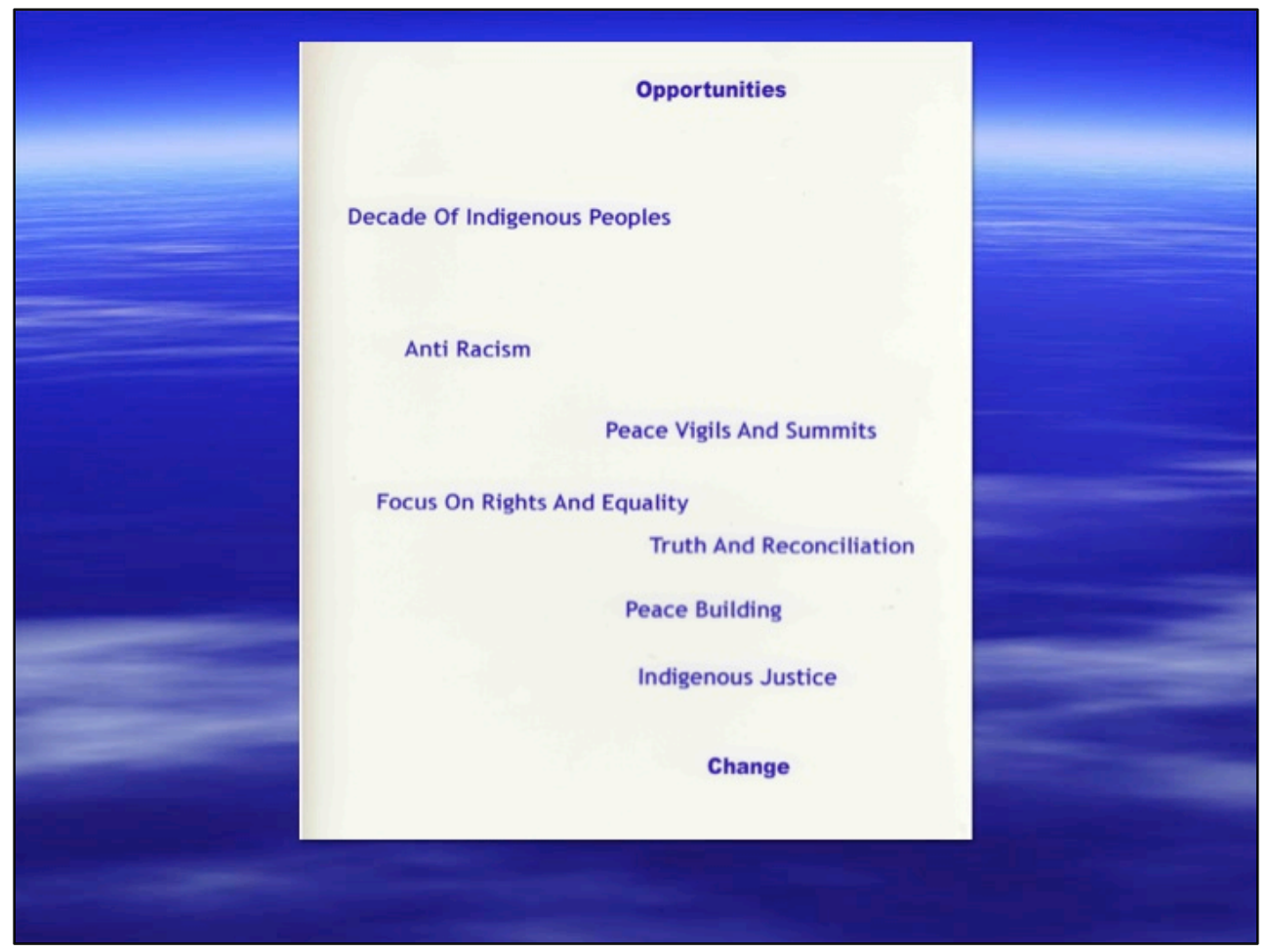

Figure 11. The Opportunities Transparency

We see hope as we explore the items on the next layer, the layer of change, of transformation. We see that there can be light after darkness, athat struggles can present opportunities. An so there are Alcoholic Anonymous programs, the International Decade for Indigenous Peoples and peace and reconciliation efforts. At the same time, we know that we frequently feel frustrated and angry that the initiatives don't always transform reality. As we realize that the first two layers, the foundation and the results, continue to be present, that they are not trapped in a past, we begin to understand why change does not come easily or quickly and why differing energies can persist and pervade across timespans. 
I liken this understanding to an apartment building. On any given night, you will find lights on in a totally random fashion, in the basement, in the pent house, and in any of the floors in between. Enlightenment is not generally distributed in a uniform fashion, and you might not find it where you might expect.

So we need to learn patience.

\section{Goals}

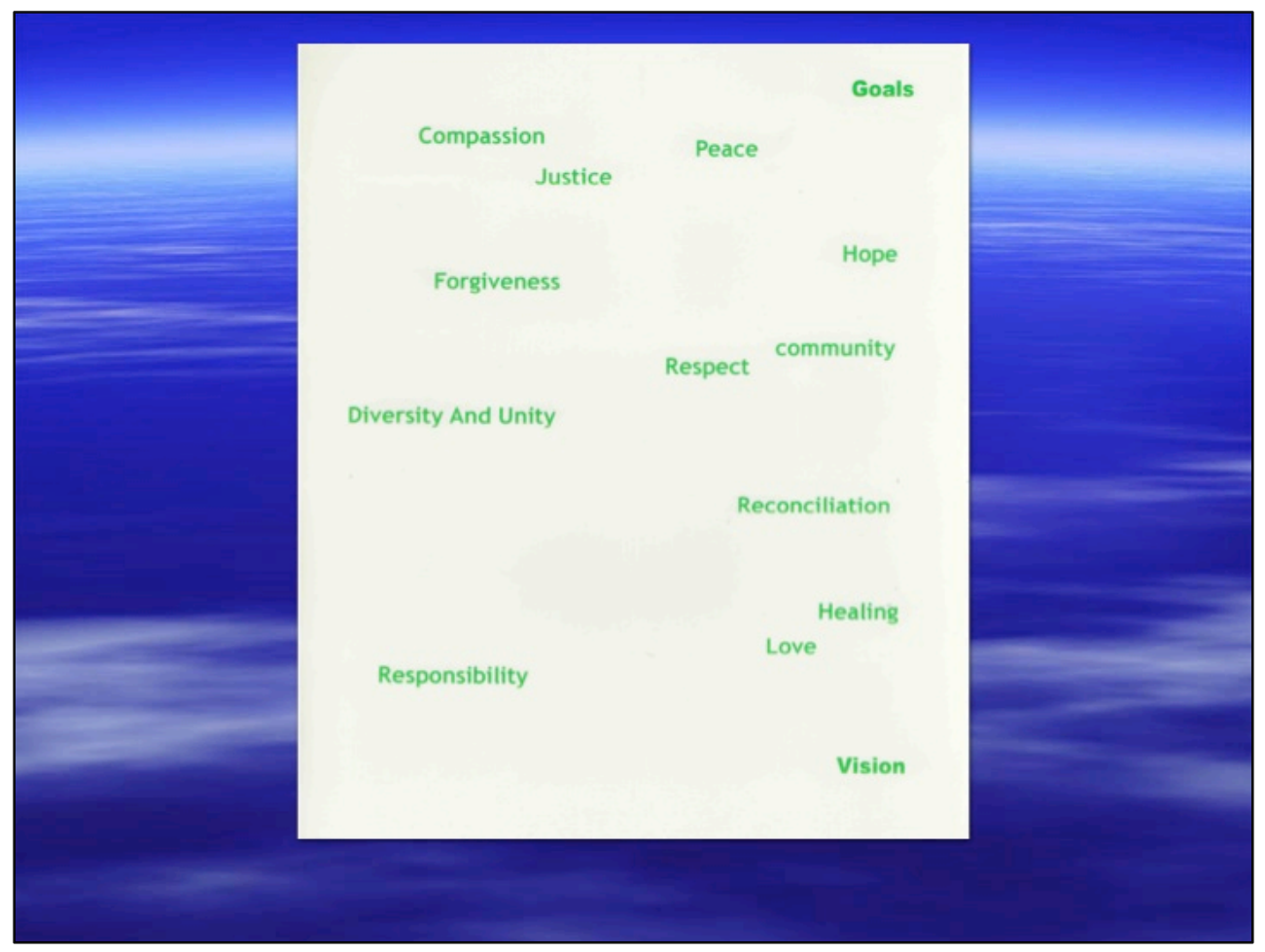

Figure 12. The Goals Transparency

The first three levels are not the good life. William believes we can go beyond. He believes that as individuals and communities, we can go beyond the limitations of our current reality.

So we look to the fourth level. Here we find the values that we hear William talking about all the time. These are the spiritually transfroming values like forgiveness, love, respect and responsibility. They come with compassion, sympathy, empathy and so on.

He believes that these values have the potential to penetrate through all the layers and to transform the quality of the experiences. So we superimpose a yellow transparency over all the layers to illustrate how healing inspired by thise fundamental virtues, common across cultures, can transform and mollify disparity realities.

As William says, we must forgive other the hardships and pain they may have caused us and we must trust that this energy will transform them spiritually. William believes that this new energy that we can generate, that in fact we are responsible for generating, has the potential 
to transform the other. And in fact, if we try to do this, really try to do this, in our personal lives, perhaps with someone who frustrates us, we find the energy shifts between us and this other. Many who have attended his Circle of All Nations gatherings year after year find this to be the case, to their great wonder and gratitude. The magic is that we really just focus on transforming ourselves and our attitudes, and enhancing our capacity for empathy or compassion, dealing with wht we can control. The rest follows in time. When you concentrate on yourself, your personal power grows.

He has learned to do this himself, and when you realize he himself carries the scars of small pox (first layer), was, as he puts it, the worst drunk on the reserve (second layer), was a tireless but frustrated fighter for freedom, for native rights (third layer to deal with the first two), but needed to transform himself and then others (fourth layer), you begin to understand why he has every faith that we all can too.

The more deeply we contemplate this ideas, and the more deeply we integrate these understandings, so we learn that we can heal from the core. As we embark on this journey, the more we find our own lives and communities transformed. We begin to see that we can become William's Circle of All Nations - Culture of Peace.

\section{Layering of Transparencies and Issues}

\section{Combination}

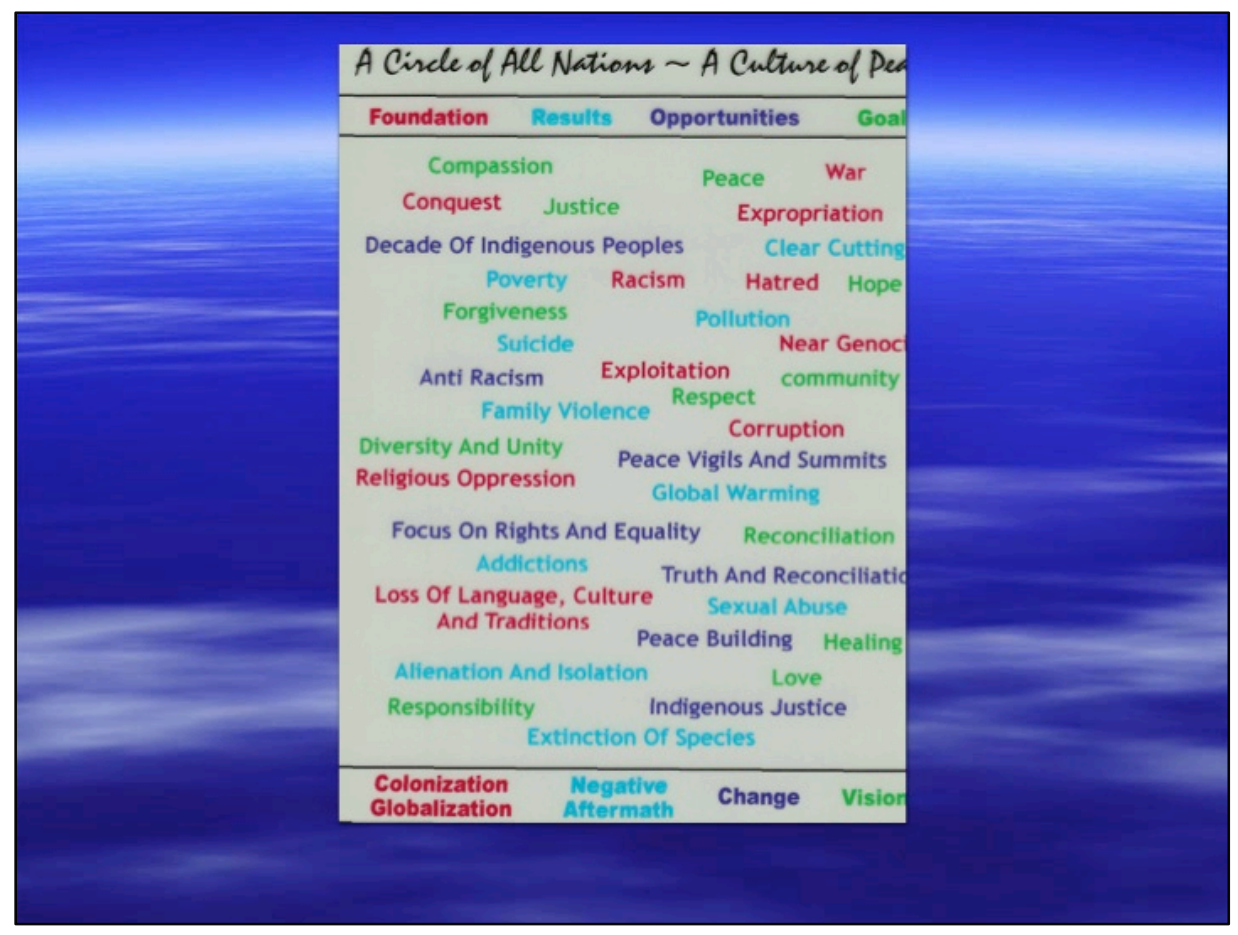

Figure 13. Layering of Transparencies and Issues

As you place one transparency over the other, you begin to realize how they coexist and contribute to the complexities, frustrations but also hope in our lives. 
He believes that these values have the potential to penetrate through all the layers and to transform the quality of the experiences.

\section{Superimposition of Transformative Values}

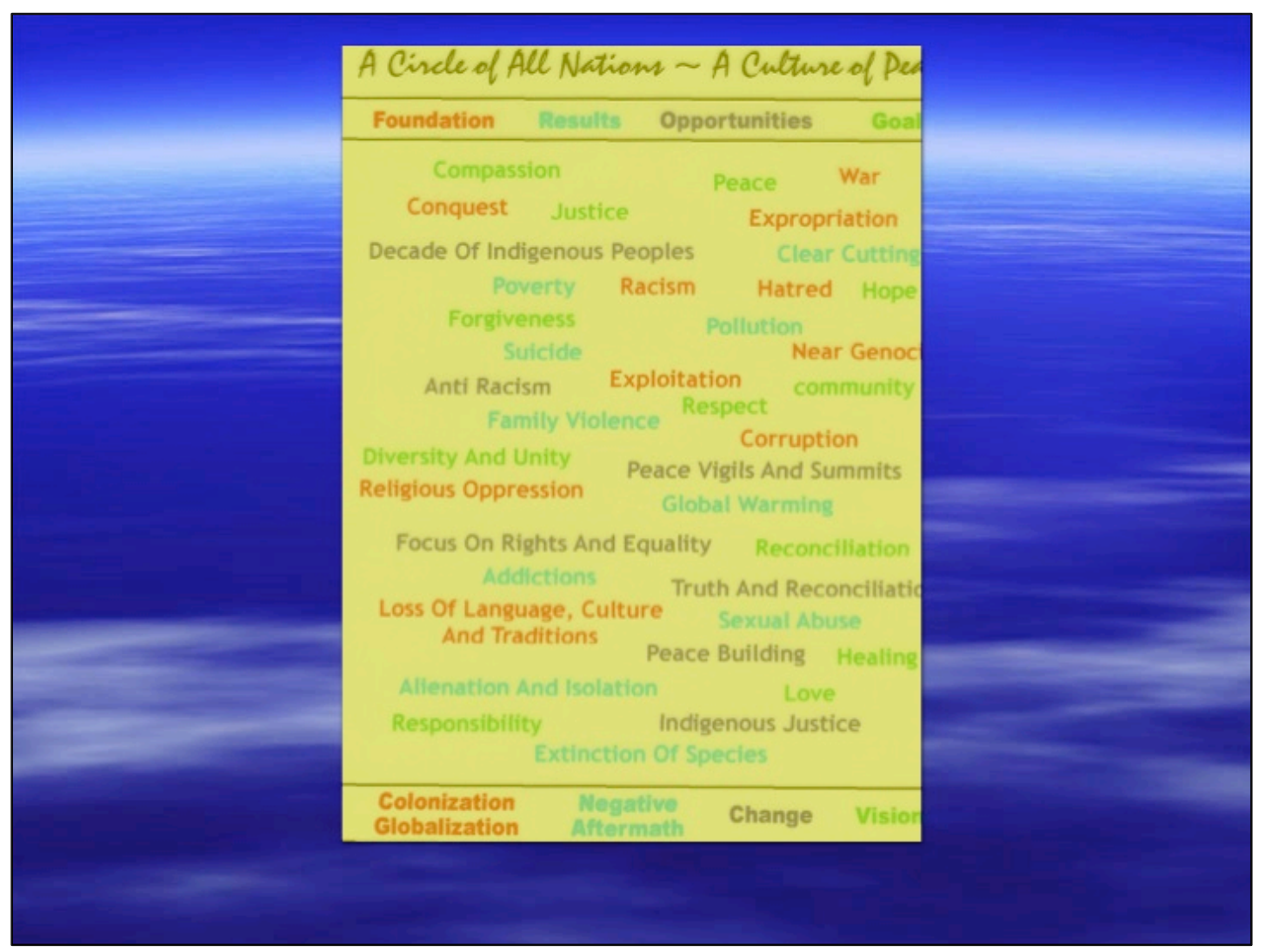

Figure 14. Superimposition of Law of Nature Energy and Values

So we superimpose a yellow transparency over all the layers to illustrate how healing inspired by thise fundamental virtues, common across cultures, can transform and mollify disparity realities. 


\section{Ideological Differences}
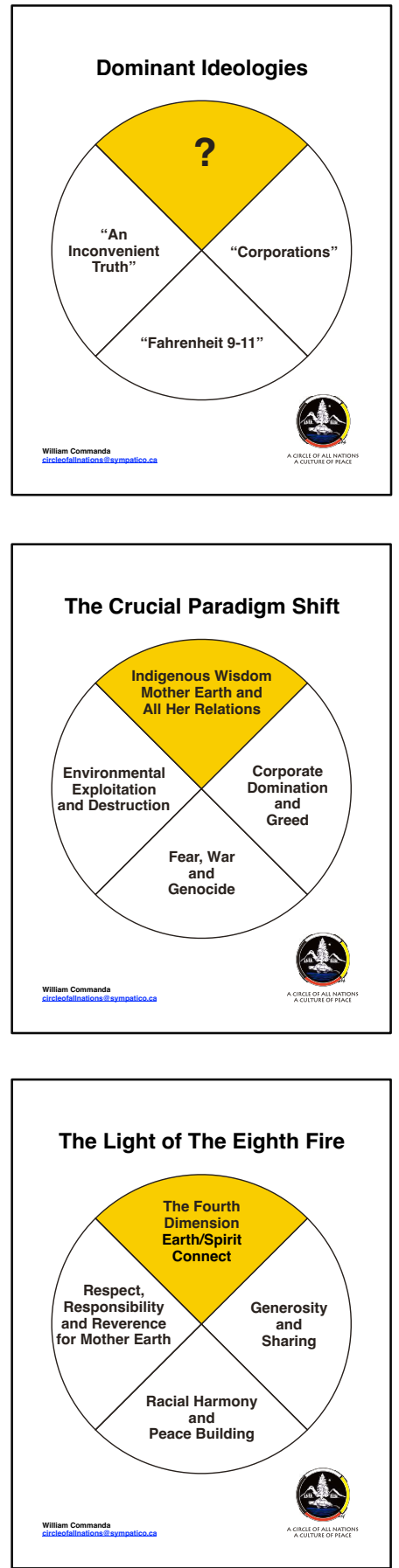

Figure 15. Ideological Differences

The following is an extract from William Commanda's speech on August 19, 2007 for the Public Service Alliance Commission Parliament Hill Demonstration against the Security and Prosperty Partnership - the remarks are illustrated with the following three Medicine Wheel 
conceptual presentations of the challenges of the day, moving from vision, to action, to review and re-visioning, consistent with the Circle of All Nations approach.

My prayer is for the igniting of Indigenous values to safeguard us all across the continent. My ancestors always knew that we were all connected, and they knew that one day, the world would have to come to us in order to understand this. Even more, they knew that oneday, the people would have to realize that they are also deeply connected to Mother Earth. Indigenous Peoples, though so few in number, have been making this statement repeatedly over the past twenty years - I believe we are the silent voice behind Agenda 21.

When the new people first arrived in the new world, they were largely motivated by greed, the realities of the day were war and genocide, and the ramifications were environmental exploitation and destruction; my ancestors were near destroyed. Today, there documentaries of the present day - Corporations, Fabrenheit 911 and An Inconvenient Truth - show that we are still embroiled in the same struggles. But now, the Law of Nature is revealing herself as the great equalizer, with ominous implications for us all and for future generations. It is no wonder that health care is the primary concern of people in this country, for life itself is under attack. But still too often we see the issues as separate. We need to realize how deeply interconnected these issues are, in order to develop a new blueprint for our collective future.

I say we need to move from greed and corporate domination to generosity and sharing, from few and war to racial harmony and peace building; and from relentless environmental exploitation and abuse to the three Indigenous Rs - respect, responsibility and reverence for Mother Earth - the values enshrined it the Three Figure Welcoming Wampum Belt.

We need to inspire others to embrace these values. First we look within to transform ourselves and, recognizing that we are all connected, we reach out to transform our brothers and sisiters and leaders. We strive to humanize institutions, a dilemma also for some of trapped within, as the phrase goes, the Machinery of Government. This is the great challenge of our times - this is what the Seven Fires Prophecy warned us about. This is the taks for which we have to strengthen ourselves.

We have to walk around the Medicine Wheel to come to the proper centre - balancing our emotional, physical, mental and spiritual capacities, individually and collectively, as we spiral towards our potential strength together. We have to inspire people to come together with One Heart, One Mind, One Love and One Determination to create a Circle of All Nations a Culture of Peace. We are now a global community. 


\subsection{Concluding Remarks}

\section{Circle of All Nations Virtual School}

The environment and respect for Mother Earth is the central aspect of the mandate of Circle of All Nations. While the Circle of All Nations also has other priorities, environmental advocacy is not seen as an independent element but is a part of a linked whole; this is an essential part of WC's approach. Hence it is different from other existing approaches to the environment in existing literature. It advances relationality concurrently at multiple levels to achieve transformational impact, not only on an external project or objective, but on the environmental activists, advocates and researchers themselves, as well as the public at large. This theme is analyzed in greater depth in Chapter 6, in the examination of the Boreal Rendezvous project. The Circle of All Nations environmental education effort is also deliberately advanced through its Facebook outreach to a largely invisible public.

Ginawaydaganuc is the all-encompassing word William Commanda used constantly, affirmative of his belief that all are inextricably connected, both people and all else in the cosmic world we inhabit, contrary to Cartesian dualistic and divisive understandings of his historic experience. Ginawaydaganuc celebrated diversity as the principle creative force in evolution. I argue that he created a discourse around this principle to contest and displace hegemonic oppression. The discursive elements cohere and maintain integrity and reflect his blueprint to greater or lesser extents; his informally constituted Circle of All Nations community animates this ontology in varied individual pursuits. Even if his students have not always understood the complexities inherent to this methodology, which meshes epistemology, ontology and ontogenesis, they have been impacted individually and collectively by multiple discursive elements in his approach to environmental stewardship and have taken threads of the teachings into their own work. This has contributed to his 
reach, where his own epistemological wheel turning from interpretation to application to replication and dissemination continues to spiral, despite the absence of a formal academy to circulate his ideas.

Over the past five years, grass roots interest in his work has served to activate tremendous interest in academic circles, in part evidenced in the Carleton University Student Union intervention and resolution on his land-based eco-peace think tank project at the Asinabka Sacred Chaudiere Site. In May 2017 fifty international academic teachers and researchers registered in the CASCA/IUEAS Anthropology Conference at the University of Ottawa participated in a field trip to his teaching lodge (where the University of Ottawa's Aboriginal Education program had been launched more than a decade ago). It was described as the most meaningful part of the academic conference, and its educational value was enhanced by the oral storytelling by his daughter, Evelyn Commanda Dewache, according to the conference convener and University of Ottawa Professor, Dr. Scott Simon. Further, WC's leadership and inspiration on critical environmental issues are acknowledged by all key environmental groups in the National Capital Region (for example, Canadian Parks and Wilderness Association, Ottawa Riverkeepers, Paddle Ottawa, Greenspace and Plenty Canada). These factors encourage me to argue that an understanding of the multifaceted non-traditional trajectories that contributed to WC's approach to sharing his historical, geographical and environmental passions can advance the inclusion of his pedagogical approach in the studies and research of Indigenous and non-Indigenous academics.

\subsection{Summary of Findings}

It is a challenge to integrate WC's storytelling way of educating people on colonial and postcolonial Indigenous issues with academic analysis grounded in dissective language. It is obvious from his narrative that WC was acutely aware of the systemic and overt racism 
and oppression of his peoples, and that until he died, he analysed the discourses of colonization, capitalism, commodification and neo-liberalism in his daily scrutiny of local and global practices and media. However, he actively and deliberately chose inter-active, animative and transformative versus didactic or doctrinal means to teach. His land-based, motional, narrative approach served him to effect over the past fifty years, and the contemporary addition of documentation in websites and audio-visual materials added tremendously to his narrative outreach and presence in multiple domains.

William Commanda animated a practice-oriented process of decolonization that is relational and reconstructive, rather than deconstructive; it leverages and promotes dialogue instead of the ontological closure that his historical experiences might have led him to accept. He thus moved his work into zone of transformative permeability and transitioning interface. Embedded in reflexivity, his practices provoke individual engagement with ideas and energies, and without stridency. In my obvious positionality as outsider, we were together able to reveal the intransigence of colonial dominance, rupture isolation and demonstrate reconciliatory inter-subjectivity. Humour was a part of his repertoire and message here; I was the real Indian not him. Also, his decolonial practices sought to achieve more just relations not only among human populations but also between humans, nature and the places we all inhabit. But with respect to people, he was acutely aware that it was not just Indigenous Peoples who were dealing with the devastating ramifications of oppression, violence and sexual abuse, as highlighted over the past five years by the Inquiry on Murdered and Missing Women, and the Truth and Reconciliation Commission and its exposure of the unspeakable abuses of the Indian Residential School era. He realized the fragility of the new North Americans, evidenced in the desperate need for personal healing he witnessed at his gatherings. He believed that unless people were emotionally grounded, they could not take 
effective action conducive to the healing of the planet. While this healing agenda is not the focus of the thesis, it was a central part of WC's environmental strategy, and its relevance grows increasingly apparent in this new age of global communications and challenge.

WC's approach to environment is a statement of resistance to convergence with neocolonizing approaches that co-opt or alter other forms of knowing, as well as to reactionary resistance focused on othering; rather, it asserts right to recreate a commons for negotiating boundaries and barriers, and creating space for bridge-building generative of creative convergence and evocative resurgence.

\subsection{A William Commanda Historical Narrative Exemplar}

An Example of a William Commanda Historical Narrative Interventions and its Trail of Influence

WC's knowledge of Indigenous and Canadian colonial history was extensive. He had a capacity to remember tremendous detail with great accuracy, and his knowledge was supported by an extensive personal library of materials, book, documentaries and research papers. In additions, he followed the daily news with keen interest.

After WC's death in August 2011, Kevin Chief, a young Algonquin from Wineway First Nations a longtime acquaintance of his, together with his music partners, created a song to a recorded recounting of history by WC. This speech is also documented in two separate videos made by two of his other young friends, Bo Dupays and Patrick Gravel. Here, WC draws attention to Indigenous lands from the time of the arrival of Columbus, asserting relationship with South America and the Caribbean Islands. In the documentaries, he also points out that no Indian was present in Charlottetown when John A. McDonald, Canada's first prime minister, carved his country into the provinces and territories. The Miskojonia Red Gold song is, as it were, the drum roll into the second interpretative lens of the 
thesis: the historical, dialectic narrative.

Miskojonia Red Gold (Flying Down Thunder and Rise Ashen One Nation (2011); (available on ITunes where you can hear Grandfather's voice).

Columbus came two times, second time he came with seventeen ships

Soldiers start killing Indian

They came for gold

When he'd seen the Pope - Alexander the $6^{\text {th }} \ldots$ - so I think what happened (is this)

The Pope told him we were not Christians: No harm to kill them for gold-

To kill us for gold

These people didn't understand Indian, and the Indians could not talk French or English

And they were shot

Try to make us lose the truth about who is our mother,

Who she is and what she does for us

They try to discourage us in every way - not to know these things

They were sending our children to the school

The schools beating our children because they talk Indian to each other

Fifty thousand children died in these schools

And my parents tell us forgive them, because they don't know what they're doing

Its been a big way - big thing

But now because of the apology of Canadian peoples - that where's the beginning -

Changes that we did not we did not have before

We were taught always to love them anyway

Regardless of what they did to us

Pray for them they said - some day they might transform to understand what they did

Its a long wait ..... 500 years William Commanda

WC was educated by and in the bush. During his youth, he accompanied his father alone to the traplines, and had the opportunity to learn intensively from him; from all accounts in the family, and as affirmed by American writer, John Durant, Alonzo Commanda had an uncanny knowledge of nature and the bush. WC's youth was impacted much trauma and oppression. This included the greed, control and the abuse of church and the abuse, racism and oppression of government, Indian Agents and schools. Finally, there was the conflictual nature of federal/provincial politics, with the former controlling the lives and the latter appropriating the resources of the Indigenous, all the while with both treating them like fools or children. WC experienced all the horrors of the overall experience of the Indigenous Peoples of North American first hand (with the exception of direct personal exposure to 
Indian Residential Schools). These included the terrorizing by the Indian agents, game wardens and police; the racism of people at large; lateral internal community violence bred of colonial oppression and manipulation, and shifting identity and status; linguistic, cultural, spiritual deprivation and turmoil; devastating poverty of the war and depression years; land appropriations and expropriations; confinement of the nomads' generative drive; humiliation; change of nutrition, injury and disease. The tough stories of abject poverty, family breakdown, unsolved death, infant deaths, racially implicated gender abuse of the most destructive nature, church control, jealousy, betrayal, theft, denial, rejection, racism, despair and alcoholism were all a part of his personal story. At one time he stated, "Sometimes, I felt it was a shame to be born Indian" (Thumbadoo/Commanda Unpublished Joint Journal 2007). I can attest to the fact that WC's stories are deeply disturbing. At every turn, he was also witness to the accelerating destruction of his Mother Earth and all her children, and as noted elsewhere, challenging this was the primary passion of his life. And yet, at the same time, bred in the bone was a thread of personal power emergent of and firmly rooted in Mother Earth, and it emerged in the flesh and then sprouted seeds of hope everywhere. This is the complex discourse that I am examining. I realize that it is a challenging chapter to address so much of history in geography. WC believed that once we were able to come to terms with our collective history, we would be able to forge to healed relations with each other and with Mother Earth, and move from language of confrontation to language of compassion. I consider it WC's recolonizing, syntropic project, igniting and spinning negentropy, with all whose paths crossed his, in an insistent affirmation of life. 


\section{Chapter 6}

\section{Theoretical Lens: Phenomenological Logic of Inquiry Seeding Negentropy and Becoming Good Enough for Two - The Border Crossing Wampum Belt and the Manashkadosh Exemplar}

\subsection{Orientation into the Phenomenological Logic of Inquiry Lens}

I use a phenomenological logic of inquiry as an entry point into the research in order to legitimize and entrench an interpretative analysis of the WC discourse and his approach to the environment through auto-ethnography and critical reflexivity. Over recent centuries, western science has dominated the environment, and WC has challenged this by animating a relational, emergent-from-earth Law of Nature approach. In view of escalating climate change and extreme weather crises, amongst other things, can his Indigenous approach to knowledge generation support or inform environmental studies?

Positivist and neo-positivist approaches to scientific knowledge were clad in the cloak of universal objectivity and conceptualized as empirically verifiable (via the senses) and analytical (consistent with their inner logic). With the quantitative revolution of the sixties and seventies, distinctions between facts and values, objective and subjective knowledge, and the practice of universalizing findings led to its dominance. With this came approaches to writing called tropes (where what is said is not the same as what is meant), writing in the third person, rendering the author and agents invisible and thus constituting an objective modality, transforming adjectives and verbs to assume argumentative power as nouns (nominalization), creating mystification, and sanctioning reification (where complex or contradictory social processes are reduced and positioned as simplified things in texts). Mansvelt and Berg make this observation: 'Writers drawing on neo-positivist approaches have shown ambivalence about their own writing practices. On the one hand, they have 
explicitly acted as if language has no impact on meaning yet ironically, they have implicitly acknowledged - as through their insistence on writing in the third person - the significant role that language plays in constructing knowledge' (Mansvelt and Berg in Hays 2010 336). ${ }^{4}$

Our values, (beyond Indigenous groundedness), were informed by poststructuralist, post-colonial, de-colonial and feminist thinking. In the academic world, Donna Haraway's challenge of the objectivity of scientific knowledge with her discussion of the God Trick of seeing everything from nowhere (Mansvelt and Berg in Hays 2010 338) and the introduction of the metaphor of situated knowledges, which entails the recognition of positionality, contextuality, embodied and partial nature of knowledge, created a significant shift. This reflects the idea of individuality implicit in the discussion in Learning from a Kindergarten

${ }^{4}$ This is most interesting to me, and it provoked this reflection: other than being a reader from childhood, and then an English student through five years of university in the seventies (obtaining a BA Hons and a MA), I was a federal government employee for twenty years, and I underwent a intensive three month residential senior management training experience at one period; my security clearance was top secret. My writing and speech writing skills proved most useful to senior public service managers and the system. This writing most definitely rendered the writer invisible utilizing nominalization and trope which permitted detached discussion or reporting on challenging secret, public security, political and diplomatic issues.

I also worked in the criminal justice system and prisons during a period when it was relatively rare to find women, let alone East Indian women, working in this male dominated hierarchical field; furthermore, women in the public service were dealing with the "glass ceiling" in the patriarchal organization, evolved, beyond its colonial roots, in a massive post WW II expansion by the employment of representatives of the armed forces (witness also the name of typical positions: directors, director general, etc. in the development of military style organizational structure of the public service; as a further aside, note too the development of geography in Canada, after the second world war - former soldiers/and new immigrants from other commonwealth countries were deployed to map the country and its resources, the Mother Earth of WC). My understanding of this reality served to nuance my style of writing.

Further, beyond childhood writing in diaries and to pen pals, I commenced journaling in the seventies, and I embraced reflexivity before it became an academic term and practice.

Separately, WC was accustomed to writing formally from the time he became chief of his community - in fact, he also typed. He was given his first typewriter by Franz X. Wagner, the American producer of the earliest of typewriters (who incidentally WC affirmed as honorary chief in the mid nineteen hundreds). WC and I first met in the context of my work in the federal government. He saw use for my writing skills in his bridging project. I note then that we utilized some components of a positivist approach to frame our objectives. I also note that sometimes the nuances WC and I got into the rhythm of expressing were more literally as compared to ironically interpreted by some Indigenous peoples, a further indicator of the challenges of cross cultural communications. 


\section{Dropout:}

Individuality is the sum of the characteristics or traits peculiar to the individual, the aggregate of characteristics that distinguish one person from others. Individuality is synonymous with uniqueness and distinctiveness. In Indigenous societies, the individual and his/her gifts are irreplaceable and contribute to the mosaic of the whole. Thus, the individual is a cornerstone of the community' (Thumbadoo 2005 24).

WC consciously created an environment for maximizing potentiality inherent to cocreative knowledge generation on themes grounded in the situated knowledges of a diversity of individuals. I suggest that in a novel way, with our two separate lenses into Indigenous and non-Indigenous, we created a shifting, hybrid, subjective and objective lens to animate the Circle of All Nations bridge-building discourse over the past two decades.

\section{Poststructuralist writers locate}

"their knowledge-defining objectivity as something to be found not through distance, impartiality, and universality but through contextuality, partiality, and positionality ... with a first person narration (that recognizes that) it is important to both reflect upon and analyze how one's position in relation to the processes, people, and phenomena we are researching actually affects both those phenomena and our understanding of them" (through) writing-in [located, partial, and situated knowledge] models (Mansvelt and Berg in Hays 2010 339).

In retrospect, I note that our books, Learning from a Kindergarten Dropout (Books One and

Two), Passionate Waters - Butterfly Kisses, and Circle of All Nations blogs of the 2000s (www.circleofallnations.ca) adopt this writing-in approach to knowledge acquisition, generation and sharing, and consciously articulate reflexivity, entrenching us firmly in this style.

Mansvelt and Berg, (drawing on Berg 1994a; Bordo 1986; Derrida 1981; Foucault 1977b; Jay 1981; Le Doeff 1987; Lloyd 1984; Nietzche 1969), offer the following thoughts in their analysis of positivism:

"positivism has both a history and a geography associated with Europe, ... is an epistemology - a theory of knowledge - that has developed relatively recently and has come to dominate contemporary intellectual life in the West ... it is dominant, or hegemonic, it rarely has to account for its own epistemological frameworks that are grounded in a number of powerful dichotomies - observation/theory, subject/object, researcher/researched, date/conclusions, and research/writing- that structure our 
understanding of research (that) arose within the long history of dualistic thinking in Western philosophy. ... These dichotomies became racialized and gendered through a long historical process of developing a singular Eurocentric and masculine concept of rational thought" (Mansvelt and Berg in Hays 2010 341), commencing with Cartesian masculinization of thought, (and) mind-body distinctions defining "appropriate forms of knowledge" (Mansvelt and Berg in Hays 2010 340).

This becomes concretized in the writing up of the research. Thus, writing is a volatile

tool.

I add Indigenous knowledge generation to post structural thinking in the following way:

Mansvelt and Berg state that

"the centrality of language expresses the fact that all processes, objects and things are understood by humans through the medium of language ... (and) we come to understand ( the material) process and objects involved through language ... and (A)ccordingly, language must be ... not merely reflective but ... constitutive of social life" (Mansvelt and Berg in Hays $2010339)$.

However, in his work, as noted in the discussion of the WC/Circle of All Nations discourse, WC introduces communicative mechanisms beyond words, textual and oral, which facilitate individual and joint knowledge generation amongst diverse players. These cannot be dismissed from critical relevance as constitutive elements as important as language. Indigenous scholar Shawn Wilson authored a provocative study of his doctoral work, inclusive of talking circles that he called Research is Ceremony; in WC's discourse, equally if not more important is the notion that ceremony is research, and much of it commences with the individual. ${ }^{5}$

\footnotetext{
${ }^{5}$ As a federal inmate, Roger Scott says in the National Film Board documentary, Ojigkwanong, (NFB Ouimet 2000) in part filmed in the prison: "For us, William is a spiritual leader, a great leader. You look at that man and you have no choice ... but to believe him, believe the words he gives to you, the teachings he gives. Seeking this elsewhere is unthinkable". He notes further, following William's discussion of forgiveness, that indeed he could do his prayers and reflections alone in his cell: moving from perhaps his history of years of prison time and the confessional box, and Hail Marys, he was now contemplating WC's profound teaching: find a place in nature to reflect on what you have done wrong (as he showed them, by gesture, that when you point your finger towards others, note you are pointing three back to yourself), then pass the guilt to Mother Earth for healing and transformation, and learn how to forgive yourself. By applying critical reflexivity to the challenge of forgiveness/pardon, he realized he was learning to forgive others, and to transform his relationships. His prayer ceremony was his research.
} 
In his discourse, WC, the virtuoso performer/teacher was at work at multiple levels, in multiple trajectories and in zones of permeability, fixing transduction, transference, transmutation and transformation. Indigenous knowledge generation includes elements beyond language. The challenge in documenting WC's unique and innovative work over the years to stimulate ongoing and emergent attention, even after his death, entails translating a sense of this into words and nuanced praxis, and modern technology in the form of photography and cyber tools are being utilized to this end.

I suggest WC continued this approach to knowledge generation in part through his canoe-making, an experientially grounded, reflexive practice; and he was deeply concerned about the increasing heavy and invasive human foot print on the earth. During an event September 2017, I observed the same practice revealed in a cross-cultural workshop. His grandson, Chuck Commanda, a somewhat shy person, was creating a canoe at a community workshop. It was only when people began to ask him questions that his detailed knowledge about the current environmental challenges impacting the life and health of many plant species was revealed. He began his study of plants with his grandparents forty years ago, and it was obvious that today, he knows more than newly trained forestry engineers and many environmentalists do. This is the type of knowledge that is critical to environmental work today. With both the canoe-school generated spontaneous teaching and learning.

Of course, the validity of qualitative research and reflexivity can be challenged. Rigour, vigilance and intellectual honesty are critical components of authentic and meaningful research. Detailed (thick) description, as noted in the glossary of Qualitative Research Methods in Human Geography (Hays 2010), and referenced to anthropologist Clifford Geertz involves not just describing an event, occurrence, or practice, but also detailing the context in which it occurs. This allows researchers to interpret the situated nature of the 
'event', reflecting on the ways in which the subject or object of study is constructed symbolically in relation to broader cultural and social relations and discourses (in Hays 2010 390). Further, a decolonial project investigates the presence of multiple ontologies, and Geertz argues that the focus of such an analysis should be on detailed (thick) description rather than on hermeneutics filtered through critical theorizations (Geertz 1973 6). This permits transformative understanding versus rote learning.

\subsection{Theoretical Frame, Methodology and Tools: Phenomenology, Auto-ethnology and Reflexivity}

Phenomenology (from the Greek word, phainomenon, appearance), at its essence, is the study of structures of consciousness as experienced from the first-person point of view, with intentionality playing a key role.

Drawing on the overview by David Woodruff Smith (dwsmith@uci.edu for Stanford Encyclopaedia of Philosophy, first published Sun Nov 16, 2003 with substantive revision Dec 16, 2013), I provide a summary of descriptive extracts of the philosophical observations to focus the examination that follows.

The historical movement of phenomenology is the philosophical tradition launched in the first half of the 20th century by Edmund Husserl, Martin Heidegger, Maurice Merleau-Ponty, Jean-Paul Sartre, et al. In that movement, the discipline of phenomenology was prized as the proper foundation of all philosophy - as opposed, say, to ethics or metaphysics or epistemology.

Basically, phenomenology studies the structure of various types of experience ranging from perception, thought, memory, imagination, emotion, desire, and volition to bodily awareness, embodied action, and social activity, including linguistic activity. The basic intentional structure of consciousness (that) we find in reflection or analysis, involves further forms of experience. Thus, phenomenology develops a complex account of temporal awareness (within the stream of consciousness), spatial awareness (notably in perception), attention (distinguishing focal and marginal or "horizontal" awareness), awareness of one's own experience (self-consciousness, in one sense), selfawareness (awareness-of-oneself), the self in different roles (as thinking, acting, etc.), embodied action (including kinesthetic awareness of one's movement), purpose or intention in action (more or less explicit), awareness of other persons (in empathy, inter-subjectivity, collectivity), linguistic activity (involving meaning, communication, 
understanding others), social interaction (including collective action), and everyday activity in our surrounding life-world (in a particular culture).

Furthermore, in a different dimension, we find various grounds or enabling conditions - conditions of the possible - of intentionality, including embodiment, bodily skills, cultural context, language and other social practices, social background, and contextual aspects of intentional activities. Thus, phenomenology leads from conscious experience into conditions that help to give experience its intentionality. Traditional phenomenology has focused on subjective, practical, and social conditions of experience. Recent philosophy of mind has focused especially on the neural substrate of experience, on how conscious experience and mental representation or intentionality are grounded in brain activity.

Traditionally, philosophy includes at least four core fields or disciplines: ontology, epistemology, logic and ethics, all clearly different, and they seem to call for different methods of study.

Ontology is the study of beings or their being-what is

Epistemology is the study of knowledge-how we know

Logic is the study of valid reasoning-how to reason

Ethics is the study of right and wrong-how we should act

Phenomenology is the study of our experience-how we experience.

Philosophers have sometimes argued that one of these fields is "first philosophy", the most fundamental discipline, upon which all philosophy or all knowledge or wisdom rests. For many, that is phenomenology.

Much Anthropological literature has dismissed the people of the American North East as mere hunter-gatherers, who left no significant monuments or evidence of their thinking or way of life on the land. This is an assertion that we strongly dispute but do not discuss in detail in this thesis. There is extensive evidence of mounds across the Continent, including the Serpent Mounds near Peterborough, and emerging research on ceremonial stone/calendar rock sites. The Vastokas team's analysis of the Peterborough Petroglyph site indicates that the early people were engaged in intensive phenomenological exploration and study of their cosmic, physical, animal, suggestive and metaphorical world. The association of the site with shaman-artist suggests that this was a priority for certain members of the community, who were tasked to study and develop the ideological framework and approach to life consistent with the relational belonging to the world of nature. The repetition of 
hundreds of images and the placement of one image on top of another provided complex evidentiary data of the intense cognitive mind-mapping study undertaken. These people learned to manage life on the land as it evolved over twelve thousand years, and to leave a very light footprint.

In view of my belief that research must go beyond the written word, I argue that we must find ways of respecting and validating the oral and non-textual means of knowledge generation and communication, pushing beyond the parameters of the poststructural. The 2017 International CASCA/IAEUS Anthropology Conference (which celebrated WC's legacy) was hosted at the University of Ottawa and drew academic attention to the theme of movement. Keynote speaker Lesley Green queried what modern knowledge might have become if movement and the lived body had been central concepts in knowledge streams, and she noted the present day importance of the thinking of the San Peoples, (indigenous to Southern Africa) to ecology. Gunn Engelsrud referenced phenomenology and MerleauPonty in a presentation on movement in yoga. Merleau-Ponty had studied the work of Cezanne, examining how the painter was able to portray nature by lending his body to the world. He argued that understanding and being able to portray the world in a painting is not an act of copying nature, but of transforming it; the artist's body is immersed in and made of the same stuff as the world with no sharp division between the sensing and the sensed, between the body and things. They are one common "flesh," and painting emerges as an expression of this relationship (from my conference notes). The canoe unifies and epitomizes this understanding of nature, art, movement and knowledge. The mobility patterns of the Algonquin Peoples of the American North East suggest that motional phenomenological priority guided the rhythm of life, and family, community and group learning practices. This went from spring time emergence on the trails of life with plants, 
fish the birds, to large summer gatherings and ceremonial exchange and practice, to fall time transition to smaller family unit hunting and trapping territories and to the months spent alone in the remote winter camp sites.

In Chapter 4, I discussed the pictographs, tools and canoe art that continued the earlier petroglyph creativity. There are other ceremonial practices that also reflect intense phenomenological exploration. The principal ones include the vision quest, sweat lodges, pipe ceremonies and sun dances which, despite government and church repression, persist to the present day. These activities support individual and group phenomenological focus, with certain elements permitting the accessing of knowledge. Some were not formally constituted as ceremonial, but were ways of being. WC recalled the poignancy and compelling nature of the lone vigil. One year, his father was kicked in the hip by a horse. Nevertheless, he had to head off alone into the bush: WC and his mother watched in unspoken pain as he limped off into the distance. Yet it was in this solitary classroom that Alonzo Commanda learned to grow fearless, and also how to read the stars and the fire. Once, WC visited Hopi Elder Martin Gashwaseoma in Arizona. He was planting corn and WC offered to help. No, he replied, I must do this myself, reinforcing the need for the solitary, relational learning practice. WC himself went off on a vision quest when he was a young teenager. This was no formal rite of passage experience, since the church had wreaked havoc on those traditional practices. It was an intensely personal response to a deep-seated drive for deep learning. Journeying, ceremony and ritual were part of the learning process, and a part of the research of nature. Unfortunately, the elimination of rites of passage experience are not only resulting in knowledge loss but also identity loss, as evidence by rising youth suicide rates in Indigenous communities and beyond. In his gatherings, WC presented opportunities for individual and communal phenomenological learning. 
Kim England's discussion in Professional Geographer of reflexivity as "the self-critical sympathetic introspection and self-conscious analytical scrutiny of self as researcher" (England 1994 80-89) can be seen to apply to both WC and myself. As researcher in his own right, he reflected on his understanding of (his) time and place, his standpoint, and consequent partiality of perspective, intuitively recognizing how positionality is "mutually constituted through the relational context of the research process" (Valentine 2003 337), this being a part of a larger dialogic process, as reflected in our Circle of All Nations understanding of individual as cornerstone of community.

Kay Anderson discusses a hybrid space issues in spaces of Aboriginality, a case study in Australia involving

"transnational exchange in contemporary cultural geography. Models of mixing that work with the idea that cultures are porous and fluid are particularly apt in relation to this case study. There is, as I have suggested, no 'pure' culture at Redfern, no crisp boundaries of inside and outside, even for so stigmatized an area. This is not only a methodological issue. It is also an epistemological problem in that the boundaries of researchable communities are not secure and areas never exist as discrete entities" (Anderson 1999 83).

The oral voice is of critical importance and the danger of it being subdued or subsumed is great. Indigenous academic writers of the past two decades now alert us urgently to appropriation and misrepresentation. I note the rendering invisible of this voice in geographical and environmental research and in multiple other fields of study, from the times of Copernicus/Galileo, and of Leopold Aldo, where the Indian is the shadow figure, to Carl Jung's insights, garnered from study of the 'primitives'. While this is not the focus of the research, I make mention of it to juxtapose and justify this in-depth exploration of the multiple facets of the WC discourse. Further, in view of escalating global environmental crises, I advocate for the end to the marginalization and minimization of the voice of the Indigenous. After my thirty years of intense exposure to and relationship with WC and many 
other Indigenous peoples, I conclude that it is critically important to pay attention to the fact that the orality itself and other intangible trajectories contribute to the earth-based knowledge desperately needed on multiple planes. Frequently, its appropriation, the marginalization or dismissal of the originators, blindness to embeddedness in privilege and patronization, and externally derived, circumscribed parameters result in profound inadequacies of the knowledge transfer and its transformational blueprint. This can also happen in well-intentioned activist efforts.

Recently, Indigenous teachers, Dr. Reg Crowshoe and Elder Rose Crowshoe informed me of an Indigenous approach to research and study that they were introducing at the University of Calgary, involving actual participation in on-the-ground tipi schools, with students producing videos both to apply for course participation and for registering end of class research findings. These approaches approximate Indigenous land-based and graphic/oral approaches to knowledge generation (Personal Conversation June 2017). In fact, I learned that in the growing recognition of multiple ways of knowing, researchers are exploring the metaphorical construction of writing as staging a text. In this regard, upon discussion of my thesis proposal with Dr. Sarah de Leeuw, she, unaware of the centrality of our Circle of All Nations tree and logo to the work, saw it formulated as a tree, with roots, trunk, branches and leaves, consistent with academic organizational ideas of theory and methodology, and research and findings (Personal Conversation 2017). In fact, to a large extent, this is how this research project has been designed, consistent with the Circle of All Nations Medicine Wheel approach. The face-book pages, cyber-atlas and social media components are conjectured as the emerging seeds of a new circle of growth and knowledge generation.

As I reviewed videos, formal and informal written materials, I realized the extensive 
process of auto-ethnology and critical reflexivity that had created WC own discourse; hence, I give primary place to his reflective voice here. However, I also incorporate my own reflexivity into the analysis, and note my own positionality. The WC teachings are embedded in stream-of-consciousness storytelling, consistent with oral traditions; message and method are co-constituted and engagement of diverse peoples in this knowledge generation process is observable over time. Today, serious researchers and environmentalists are finding that Indigenous knowledge is increasingly relevant for environmental research and multiple other global challenges. The pentimento layering of oral storytelling ensures deep and transformative learning; it was a methodology of Circle of All Nations and both the rationale and its use will be presented in this section of the thesis.

With respect to myself, I have been intensively engaged in Indigenous issues for over thirty years, and in William Commanda's work for over twenty years. I hold a unique insider/outsider status in his work. My formal work history over the course of four decades lies in social justice, Indigenous justice, federal public service, security and social work, and I utilized storytelling extensively in this domain. My academic background emerges from a study of literature: English, Canadian, American and South African. The use of text, symbol, semiotics, based on field and participant observation, nominalization and positionality have all played critical roles in my evolving work, and in both knowledge acquisition and knowledge generation. My search for appropriate methodological tools to examine the unique, bridging discourse of WC and the Circle of All Nations led me to conclude that autoethnography and critical reflexivity would contribute best to its interpretative analysis for this part of the analysis. As noted in the chapter on methodology, I adopt a storytelling approach to substantiate and inform the research.

Auto-ethnography is a qualitative method involving the explicit writing of an 
embodied and situated self into the research. It often entails writing in a literary fashion, involving autobiographical narratives, wherein the author/researcher actively reflects on personal choices, emotions and knowledge as a vital part of the construction of the research. Ellis discusses auto-ethnography. He defines auto-ethnography as a research, writing, story and method that connects the autobiographical and personal to the cultural, social and political; auto-ethnographic forms feature concrete action, emotion, embodiment, selfconsciousness, and introspection portrayed in dialogue, scenes, characterization, and plot. Thus auto-ethnography utilizes the conventions of literary writing (Ellis in Hays 2010 351). Ian Cook advocates to students the use of auto-ethnography as a means of writing reflexively and critically, bringing the insights from their reading, visual, and aural material research and their felt experiences, emotions and knowledges into their assessment on geographies of material culture (Mansvelt and Berg in Hay 2010 352). In the use of literary rather than objective modalities, writing becomes a method of knowing provoking an awareness of situated problematics and politics of knowing, representing, and transforming knowledge. Cook suggests further that this can have a radical effect beyond any didactic connection between what is known and how it is interpreted. In this context, my earlier academic studies fell in the domain of literature, and while not expressed in the language of discourse analysis in the seventies, my thesis did in fact reflect an engagement in this approach, in its examination of music as constitutive of the underlying discourse that led to political change in South Africa, as reflected in the novels of late Peter Abrahams. Just this year, a master's student in Canada contacted me via Facebook to say that she found that 1976 thesis helpful to her understanding of discursive issues. I have also written three books on William Commanda and the Circle of All Nations work. This literary background lends itself to operationalization in my current research project of analysis of WC's discourse. 
But I suggest that auto-ethnography can be powerful beyond writing. Later, I examine how WC's canoe-making documentary, Good Enough for Two, becomes a school of knowledge and a vehicle for exploration of ideology, subjectivities, power, truth and meaning in a journey across time and space. It constitutes a way of knowing and as such, its role in the WC bridge-building discourse draws the domain of creativity and art expression into the repertoire of Indigenous knowledge creation and teaching. Its validation as a multifaceted Indigenous epistemological technology, and not merely a craft exposition, will be examined in our query into any ongoing relevance of the WC discourse within the academy.

Critical reflexivity is a process of constant, self-conscious scrutiny of the self as researcher and of the research project: Dowling states that "being reflexive means analyzing your own situation as if it were something you were studying. What is happening? What social relations are being enacted? Are they influencing data?" (Dowling in Hay 2010 37). The exhortation to examine ideas and thoughts about the research process, its social context, one's role in it, outsider/insider attributes (inclusive of racial, ethnic, religious, gender and socio/economic/education/employment elements), positionality, partiality, contextuality, assumptions, power relations (asymmetrical ones) are critical to credible research and intellectual integrity. At the same time, Dowling also notes that subjectivity involves the insertion of personal opinions and characteristics into research practice and that this is relevant and potentially enriching because qualitative research methods involve social interaction. Thus, rapport, communication skills and awareness of the specific social context, semiotics and issues help to decipher text and discourse.

This excerpt from my journal entry after my first meeting with William Commanda on April 11, 1997, attests to the long practice of reflexivity fundamental to our relationship and to my critical role in the relaying of his story as he intended me to. I wrote this in our joint 
journal, reflecting on my earlier personal journal:

\section{An unlikely connection}

In the intensity of our initial connection, there was already a sense that I would be able to breathe some of it as he had, that despite the gulf, the immense chasm separating us, each coming from such vastly differing worlds, (you know, the barriers of language, experience, ideologies, country, age, gender, race, work, background, upbringing, education, culture, religion, let alone the hardening in my bureaucratic veins, etc.), that I would be able to see it as he had, and in the envisioning, in the revisioning, transform my understandings, my memories, my life, and yes, my responsibilities... Four years later, ... I see that in fact, I have been coming home to myself; in understanding his Indianness I have actually come into my own.) ...

I gradually came to understand that what he was sharing with me was not for me alone. And finally I accepted that William wanted to share his story with you, and that he had assigned the task to me.

Many times I think the task is beyond me; but at other times I know that he has taught me to follow the threads. Now I have to braid this rope well to tie you securely to him (Thumbadoo/Commanda Unpublished Joint Journal 2007).

Further, inter-subjectivity plays out in the meanings and interpretations of the worlds created, confirmed, or disconfirmed because of interactions (language and actions) with other peoples within (a range of) specific contexts (Dowling in Hay 2010 35). My cursory review of my notes and tapes of discussions with WC reveal that he too engaged in a process of intense reflexivity, both in his personal style and growth, and in his discussions with me, developing, refining and highlighting themes or ideas of significance. I note that language plays a critical role here, and there is unfortunately a tremendous loss in that he was not communicating in his first language to someone who could understand it. Instead, he translated his mind along a pathway that leapt from the land to Algonquin to French, before it turned to English. Here it is worth pointing out the French learned via the Catholic priests and monks favoured a interpretative "spiritual" lens distinct from the secular English, which from time to time informed his articulations, and then at others, was consciously rejected in the deliberated creation of his own discourse. 
In the case of the Circle of All Nations thread, there is a vast range of inter-subjective moments for analysis involving a larger range of voices. In bridging the cross-cultural world, I bring a particular and irreplaceable capacity for reflexivity, given my unique history and relationship with the work. Dowling points out that inter-subjectivity means that "neither you, your participants, nor the nature of your interactions will remain unchanged during the research project" (Dowling in Hay 2010 37).

Mansvelt and Berg offer a comprehensive overview of the findings of several researchers with respect to constructing meaningful geographical knowledge, and this orientation serves as a lens into the reflexivity focus in this section of the thesis (Mansvelt and Berg in Hays 2010). They reflect on objective knowledge, once seen as scientific, rigorous, detached and consequently valid, being challenged as in fact being monolithic, universal and totalizing, with such objective knowledge operating on rhetoric's of disembodied rationality and transcendence to attest to its truths (Mansvelt and Berg in Hays 2010 335). They also discuss third party narrative constructed as objective modalities, with the transformation of adjectives and verbs into nouns creating mystification and concealment and reification, where uncertain and contradictory processes are assigned the more certain status of things (Mansvelt and Berg in Hay 2010 336). The use of the third person is illusionary, they point out, negating acknowledgement of the ways in which power and subjectivity are constituted through the research process.

Research is a re-presenting of findings, through a particular medium, writing, inclusive of its constitutive, dialogic and signifying character. Critical reflexivity demands the selfconscious analytical scrutiny of self as researcher (Mansvelt and Berg in Hays 2010 344). Since our discourse evolved over rapidly changing times, the phenomenological approach will ensure that I track the shifts in modulations of the discourse with considerable care. 


\section{Methodological Tools}

A self-conscious reflexive examination of the deployment of multiple pathways to knowledge acquisition and knowledge generation in this Indigenous discourse will safeguard against reliance on written words for any presentation of a full picture. Here, my research sources include not only written, photo, audio and video material, but also rich experiential, journeying, ceremonial and conversational material, under constant reexamination, memorialization, and reanimation, for both myself and multiple diverse audiences. However, this library is also huge, after twenty years of writing, photographing (over 40,000 in this age of the digital camera; audio and video recording (WC was recorded on multiple occasions, both formally and informally, as evidenced by any Google search on him); and Facebook messaging, where he remains encore vivant.

While some of this material constitutes the backdrop for the NVIVO and digital atlas analysis, I have selected the following items for examination here. Consistent with the storytelling orientation and medicine wheel flow, after this contextual discussion, I reflect briefly on my summer school of 2008, kayaking and studying at WC's lake side home, and the dissertation in the form of my photo journal Passionate Waters - Butterfly Kisses: Summertime Kayak Journey on the Parallel Lake. Then, I address certain themes of the Seven Fires Prophecy and Border Crossing Wampum Belts and the National Film Board documentary, Ojigkwanong - Encounter with an Algonquin Sage, excerpts from our Thumbadoo/Commanda Unpublished Joint Journal 2007; and our Good Enough for Two canoe-making documentary. I conclude with a WC video clip, and audio clip, and an audio-recording of me being interviewed by Prophecy Keepery, with no further textual analysis, to permit the reader/listener/see-er to engage reflectively with the discourse. Again, the idea with the cyclical approach is consistent with the Circle of All Nations project of moving from a vision/planning platform, to an 
action/animation zone, to a deeper reflection; to an articulation of understandings, in order to propel into another level of the spiraling journey of knowledge generation.

I have commenced an extensive examination of the storytelling approach to relationship building and knowledge generation in the work of the late Indigenous elder William Commanda (1913 - 2011), as animated and vitalized in and variety of digital tools and cybercartographic flow. An evolutionary, distinctly human capacity for storytelling has re-presented itself on the global stage in a massive social explosion. In outpourings of volcanic proportion, storytelling is creating new ground for the presentation and performance of an infinite number of joint and networked dormant and emergent narratives. Digital technologies are magnifying this reach, upsetting dominant paradigms and knowledge bases. William Commanda was born at the beginning of the last century, on the first reserve created in Canada. He is the longest known guardian of the ancient mnemonic Wampum oral storytelling and record keeping heritage of his Algonquin ancestors. He was born of oral tradition, and raised in the generic prototype of the Algonquin linguistic tradition. He also learned to speak and write in French and English, and storytelling was his forte. He created a global eco-peace community, the Circle of All Nations, and he relied heavily on oral storytelling, talking circles and other semiotic and semantic mechanisms to advance crosscultural understanding, bridge-building, information sharing and relational change on environment, social justice, healing and peace issues. Over the course of his lifetime, cameras and video recorders extended his reach and influence. In the 2000s, he became a presence in social media, known popularly as the dot.com elder. The cybernetic loop in his storytelling practice repeatedly draws new voices in. Cybercartography will permit further exploration and development of his approach. Its innovations support an authentic exposition of 
William Commanda's singular discourse, and animate the epistemological objectives and technologies of his outreach.

\subsection{Conceptual Medicine Wheel Orientation Map}

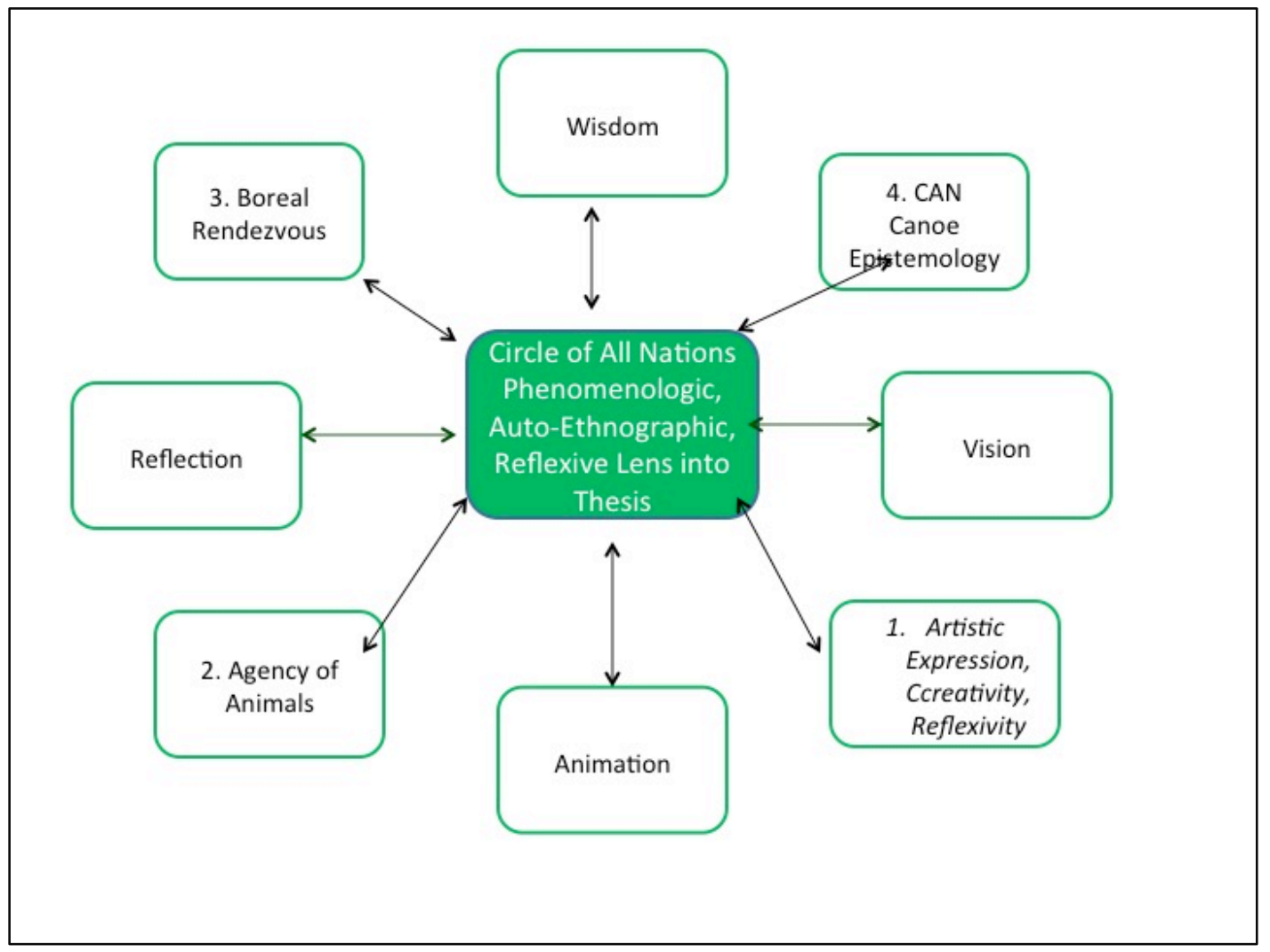

Figure 16. Conceptual Map of Circle of All Nations Phenomenological, Auto-Ethnographic Reflexive Lens into Thesis

\subsection{Discussion, Analysis and Observations}

\subsubsection{Artistic Expression, Creativity and Reflexivity}

The most ancient example of a nature-based spiritual art study site of the Algonkians can be observed in the tool making schools across the land. Morrison Island is one acknowledged archaeological site on the Ottawa River Watershed, near Ouiseau Rock and the rock paintings, Allumette (fire) and Calumet (pipe) islands, and several notable falls and rapids. Many stone tools and artifacts were also found at Lac Desert, the ancestral lands of 
WC's grandfather Louis Commanda. Here a line of seven large turtle shaped rocks guard a strip of shoreline. Most recently, in 2014, thousands of artifacts from distant locations like Lake Superior, New York State and Northern Quebec, dating back eight centuries, were uncovered at the Jacques Cartier site on the Gatineau. This was followed by Indigenous protests and activism to protect the area, still unresolved legal challenges, fragmentation of Indigenous voice, (including with respect to WC's vision for the Asinabka Sacred Chaudiere Site), and serious flooding in the spring of 2017. Their presence serves as further confirmation of the significance of the Ottawa/Gatineau/Rideau river cross roads meeting place as a critically important spiritual art creation, vision questing school site.

Some of the stone site findings reveal the distances covered and the mapping knowledge of the ancient peoples; others confirm the workshop idea. WC is directly linked with affirmations of this heritage. For example, he accompanied archaeologist Bill Allen to the Giant Turtle site on Catfish Lake in 2005, with representatives from Parks Canada and Algonquin Park. This site, a place of convergence of canoe routes, marked by the presence of the giant Turtle Rock at the point, Makanak Assini Neiashi, was also the site of a mica and stone tool making school. WC conducted wampum ceremony. Federal and provincial government representatives participated in the ceremony and learned from the experience and reported on it. WC pointed to the cosmic orientation of the Turtle Rock. The island was named Ojigkwanong, and affirms WC's energetic presence; but it is also important to note that Indigenous place names are descriptive of the sites themselves. The giant rock affirms an earlier statement I made about the use of ice for the creation of rock sculpture by the ancient peoples. The photograph of this rock from the nineteen twenties shows that it was resting on smaller rock feet.

The WC/Circle of All Nations knowledge generation reflexivity is in essence also much 
like the practice animated by the ancient Algonkian peoples at the Peterborough Petroglyph Site, where over an extended period, countless people repeatedly engraved hundreds of both recognizable and today unrecognizable images, on a massive table of limestone fed by an underground stream. To WC, this was the principal school of reflection, knowledge acquisition and knowledge generation attuned to the spiritual energy of the universe. These emerged both from and in the evocative rock formations. The Vastokas team notes the importance of relationship with land and reflexivity:

"The relationship between the artist and his medium revealed at this site is one of total cooperation between man and nature; the artist works with, rather than imposes himself upon, the natural environment, a fact that discloses a great deal about the artist's culture, about Algonkian world view and mythology.... If the glyphs are to be experienced as being at one with the rock both physically and iconographically, then the site itself must be perceived as integral with its landscape setting and surrounding space. In Algonkian ideology, as we have seen, space is not without form, it is charged with meaning, structured, and differentiated in terms of axial directions, sacred centres, and degrees of supernatural potency. The choice of the limestone surface... attests to the fact that the rock itself is a sacred object and embodies in itself a concentration of Manitou (spirit). It is also the microcosm of the universe, a spiritual sanctuary, and archetypal temple, (indicative of) the Algonkian sense of place, their feeling for the potency, oneness, and sacredness of natural phenomena. This form of sacred art is an outgrowth and expression of reverence and respect for nature in all its manifestation, for nature's landmarks of special and spiritual meaning ... (evocative) of the very structure of the universe ....The images engraved here are not the projections of man, imposing his will upon his surroundings. Instead they render visible the hidden meanings in nature whose significance it has been the shaman's task to conjure* up and capture on stone (Vastokas 1973141 - 142). (*conjure as in summon, call up, invoke)

Images from this site may be seen in another important Algonkian art form: in the pictograph sites across the Canadian Shield. These rock paintings, using red ochre and fish oils, with fingers for direct contact brushes, were executed on rock cliffs, accessed from a canoe or ledge, in selected sites. In the Ottawa River Watershed, a few notable sites are the Lac Temiscaming Spirit Rock, the Ouiseau (Eagle) Rock site near Pembroke (close to the ancient red ochre quarry on the Mattawa); one in Lac Simon (Chimaan/Canoe) near the Lac des Etoiles, the ancient Algonquin Milky Way observatory; and another in the 
Baskatong/Mishomis area, close to the Windigo Rapids, a natural waterslide and ancient vision quest Spirit Mountain site, re-named Montagne des Diable. I argue that the artistic and creative forces are aligned with the spiritual, and the association of the pictographs with land (rock and water) features are statements of the Indigenous affirmation of spiritual relationality with the forces and Laws of Nature. This is what is construed as sacred in the English language.

A third form of comparable art expression was invoked in the birch bark canoe. They these were also serviceable material items that they were designed to negotiate water and rock. They affirm Algonkian relationality, mapping and mobility in the emerging landscape of tumultuous water and rock after the retreat of the continental ice sheet and affirm WC's locatedness in the Ottawa River Watershed. As such, he pointed out that it was the Algonquin peoples who created the birch bark canoe to navigate the rocky waterways of Northern America; others, like the settled Iroquois tribes made heavier dug-out canoes. Further, the birch bark canoes were also canvas for iconic creative articulation of relationship with nature. Not everybody made the effort to secure the winter bark that could be etched upon. WC created stencils of the symbols of nature to mark his canoes reflexively. He was part of an ancestral line of shaman/artist/teachers, and as a child he apprenticed with and learned from his Uncle Andre Cayer, descendent of the Lac Simon/Chiman canoe maker. Lac Simon was a site marked by pictograph. WC later operated a canoe school at his home, teaching his grandchildren this nature-based, relational knowledge generation practice. At age 90, he engaged in another canoe school project; the documentary of this experience, Good Enough For Two, will be analysed later in this chapter.

A similar canvas educational tool played a significant role in WC's Circle of All Nations school of co-created reflexive knowledge. Under the leadership of artist and innovator Derek James, art panels were co-created on fabric by a diversity of participants. These draw art, 
social justice and activism themes together. They explore diverse topics of WC's work such as the demise of the Beothuks, Murdered and Missing Indigenous Women, William Commanda's vision for the Indigenous Centre at the Sacred Chaudiere Site, and peace building. These were co-created at Circle of All Nations gatherings, including at its international millennium peace conference, and at rallies for the Sisters in Spirit, (the initiative that first drew attention to the Murdered and Mission Indigenous Women), and First Nations Poverty on Parliament Hill. ${ }^{6}$

At this juncture, the key point being emphasized is that through the reflective process observable at the Peterborough Petroglyph Site, the ancient people reveal that they had developed a nature-based relational knowledge acquisition practice. WC himself consciously and determinedly continued to animate this for himself and others throughout his own life. This reflexivity is deeply generative, and for WC, it was the means to challenge entropy. This motif, as illustrated by the Beothuk example, is persistently embedded in his discourse.

\section{Passionate Waters - Butterfly Kisses: Summertime Kayak Journey on the Parallel Lake}

In 2007/2008, a community group from Kazabazua asked to hold a meeting in WC's teaching lodge at his home in Kitigan Zibi Anishinabeg, Quebec - they wanted to challenge the development of a mega-dump at Danford Lake. In the spring, following much research, community engagement and ceremony to protest the expansion of the damming at the Chaudiere Falls, uranium mining at Ardoch/Sharbot Lake, and road expansion destructive to

\footnotetext{
${ }^{6}$ A way to trace the knowledge generation hodological process (Turnbull) might be through the story of the Beothuks. WC noted in the historical constitutional meeting of 1987, that the Seven Fires Prophecy Wampum Belt had alerted the Algonquins to the impending demise of the Beothucks with the coming of the new people; Derek James, a non-native artist and Circle of All Nations colleague originally from Newfoundland then created a banner to share their history, and organized a medicine wheel prayer for them at the ancient Quarry Site in Ottawa to raise awareness in the capital city; over recent years Chief Mi'sel Joe, his Newfoundland friend, has been involved in a challenge to repatriate bones of two Beothuk from Scotland, the federal government has become engaged this effort during the past year, and this historical initiative and the genocide was profiled at the Circle of All Nations 2017 fall peace event, twenty years after WC original public statement; plans are also envisioned to create Mount Beothuk Historic Site.
} 
the migratory path of the iconic Loon, I now had to research the potential environmental impacts of mega dumps; (note that I came from work in prisons, and knew very little about dumps). WC, however, was determined to buy me a kayak. He knew that my real education about water had to come from $W$ ater herself. Eventually the experience translated into a photo-journal: Passionate Waters - Butterfly Kisses: Summertime Kayak Journey on the Parallel Lake: my quest for water spirit on the Parallel Lake:

I began my summer journey entering a new chapter of William's story - exploring his lake. By the end, I found I had come home to a sharper connection with my own interior world. Everything penetrated me deeply, in this interface with the parallel reality that holds so many of the deeper truths of life. Dedicated to the return of the Eel Spirit, I share these photos and thoughts to give you some glimpse into the transformative experience that awaits the awakening spirit, perhaps right on its own doorstep within Mother Earth's elemental world (Thumbadoo 2008 2)

After my initial humbling experience of tipping my kayak into the mucky, froggy water, I kayaked passionately all summer, and learned empirically and through intense observation, about rocks, trees, water lilies, turtles, beavers and beaver cabins, water snakes, otters, mink, eagles, loons, kingfishers, current, temperature, fog, moon, stars, dawn, light, sun. I felt guilty all the time about all the things I ought to have been researching for our many Circle of All Nations projects, but day after day, I went out earlier, and returned later. I took many photographs, to show WC what his lake was now looking like; then suddenly I realized that I needed to create a photo journal, to document and share the pathway to knowledge and grounding that I had accessed through my intense exposure to nature. After that summer, I felt cleverer than ever, my brain felt alert every minute; it was as though nature herself had exfoliated dullness from my brain. I produced the Passionate Waters - Butterfly Kisses: Summertime Kayak Journey on the Parallel Lake journal to present this doorway to nature to others and to detail evocative nuances of learning via the lens of nature process. Nature, 
water, light, wind, bird, movement, camera and I were intermeshed. ${ }^{7}$

${ }^{7}$ Further Reflections on Passionate Waters - Butterfly Kisses Summertime Kayak Journey on the Parallel Lake to further substantiate discussions on the Law of Nature.

William took this photo the first day I headed into the lake. I was already wet. I had tried to get into the kayak from the deck by myself, and in a split second, my kayak flipped, and I was immersed in the mucky, muddy, froggy water, with a bullfrog eying me contemplatively; and my perfect new red kayak was baptized with mud! Now, I had skirted that spot gingerly for a decade, without getting a toe wet - but Parallel Lake knew I needed a sharp shift in perspective to set me on the right track for a meaningful experience on its waters. All my squeamishness dissipated, and suitably bumbled, I took off into the blue. p. 2

There was so much magic and mystery in the melding of the branches and leaves and water and light, particularly when I let the kayak and current take me where they wished - and the images I saw invariably impacted the separate thoughts or issues I was working through, deepening my insights and explorations. I love journaling - it is like talking to my deeper self, and I consider it the greatest tool to true learning; now this was like talking with my deeper self, but enhanced by its interface with the enigmatic energy of nature. I kept wanting to say, thank you and acknowledge something - my paddle strokes became like gestures of gratitude and reverence. $p .28$

If we do not find our rightful places under the Sacred Tree envisioned by the visionary Black Elk, then the First Peoples of the land will remain displaced. Will the land accept its step children? Perhaps, as William often muses, if we remember that we were welcomed here by his ancestors, the original caretakers of the land. The blood and bones of his ancestors are deep in this land. He is "at home" here. Are we? We have already severed ourselves from a sacred connection with the land of our ancestors, myself, at least twice. How can I possibly understand his link? p. 32

He reminds us constantly about his intimate relationship with Mother Earth, the living Gaia; for us she is Environment and Commodity, and we are detached. If we do not form a relationship with him, and learn to reconnect with Mother Earth, can she really embrace us? Already there are ominous signs that she has begun to shrug us off. An engineer and technician from Portland Oregon discovered William's website, and wrote about how moved he was by these lines in T.C. McLuhan's book from the sixties, Touch the Earth: "How can sprit earth care for the white man? Everywhere he has touched her, she is sore." It was a chilling premonition - just look at global demography. p. 32

On the evening of the July Full Moon, I was on the other side of the Rocky Point. The water was perfectly still. By itself, the kayak shifted direction. By now I had learned to go with the flow of the water or wind at times - but this was different. Everything was dead still.

Gradually, my eyes focused in the dimming light in the direction my kayak pointed, and there were seven loons; a few minutes later they trilled their song to Grandmother Moon. I captured it on a short movie clip ... moments later, nine loons headed out into the great blue above to meet Grandmother Moon. Loons are 60 million years old, I was told, the oldest bird on the planet. I asked William about my kayak's shift in direction - Its energy, Romola, energy. In fact, that is all it is about - the ancient energetic cycle of life. p. 8

Days on the lake were glorious. But nighttime brought a deeper magic. Then, lake and sky were inseparable and it became easier to understand what William means when he says everything is connected. The stars descended to kiss my kayak. Sandpiper lingered on the shoreline, as reluctant as I to abandon the exquisite intoxication of the night smells, the subtle colours, the faint whispers, the shift in the wind, the cool, silky water - we were immersed in a sensual experience of our world - how much more I understood now about one of the pathways to knowing - thorough the senses - me, who generally lived through my head. How rich and expansive it was. p. 44

I recalled the ancestral memories of Giant Beaver creating the land of the Mamiwinini in this part of North America, laying out the pathways for the journeys of the nomadic peoples over a land of lakes, rivers, rapids, dams, islands and trees. These created the vast boreal forest which plays a central role in ensuring the bealth of the entire planet. Within the tracks and tales of William's own ancestors, we have retraced journeys from the salt waters of the Atlantic along the mighty St. Lawrence, the wild Ottawa, through great rivers and portage routes, to the greatest fresh water lakes of Mother Earth. Now, Beaver Spirit was guiding me to new understandings of its word. $p .11$ 
I used to sketch images of clearings in nature during the period I kayaked on WC's lake. Recently, I noted Heidegger's discussion of Being: That which is can only be, as a being, if it stands within and stands out within what is lighted in the clearing (Heidegger 1977). It is only within the clearing that things are made visible. Heidegger's clearing is framed by colonization and European thinking. Critical geographers have examined the construction, materialization, commodification and reification of nature intensely over the past two decades. While he did not necessarily discuss the construction of nature with the academic terminology, WC was acutely aware of their significance; he hosted his own gatherings to bring the wisdom of Indigenous elders to bear on the escalating environmental crisis. WC's Law of Nature heritage saw both the world of the clearing and beyond, and he contextualized this in Ginawadagunuc style, in cosmic space and time. Like Morning Star, bridging between Night and Day, he was determined to throw light beyond the clearing of non-Indigenous discourse. With his affirmation of Ginawaydaganuc, WC worked hard to make all people his relations, to thereby ignite a living relationship with his Mother Earth, relationships of being

I am regularly attracted to certain rocks and stones; most people are, without really understanding or reflecting on this magnetic pull. Indigenous Peoples have studied this over eons of time. The native way is to honour the connection and to put down some tobacco in exchange for the stone and whatever it can offer one - that is how the great cycle of energy circulation and balance is maintained and replenished. It is not just precious stones that hold power. Those have been so commodified, they have become emblems of artificial power. Many have found the humble stones they find along their pathways the true power brokers - on the road to personal power and wisdom. p. 53

Over the next few weeks, I was to discover all the Beaver cabins on the lake, each one so different from the others, some old, some new, some in the process of being renovated.... I final began finally to understand what William had been trying to teach me about how the beavers create their lakes and dams, how they manage the water levels to support life and biodiversity, and how they create the features on the face of Mother Earth - now, finally, I began to recognize the difference between the shorelines created by those co-creators of nature and those bulldozed into the resisting earth. $p .14$

Later in the season, at this spot close to the den, I found two young beavers! Indeed a den of procreation. You can imagine what a thrill that was. William thought I would trap them for his dinner - I threated to park myself on the lake to protect them! - Grey Owl - mover over! (We had celebrated the conservation efforts of Grew Owl, Anahereo, his wife of Algonquin ancestry, and Uncle Gabriel Commanda, who knew both of them, in November 2006, sixty years after their petitions on behalf of the beavers, - now, I who had lived so close to Grey Owl's home in Waskeisu Park, Saskatchewan, was finally making acquaintance with beavers, on Uncle Gabriel's lake!) p. 22 
and becoming; he believed that only when humans embraced such a relationship would they be in alignment with the Laws of Nature, and advance balance and environmental stability for all.

I began to realize that for WC, from his lifetime of study, the cognitive and empirical evidence that he had secured suggested that the settlers on his Mother Earth had no relationship with her, detached from her physical corporal body; thus, they were capable of indifference and abuse to her; he theorized that if he made relatives of the newcomers, they would then develop a relationship with Mother Earth too, not materialize her and view her merely as resource and commodity, but recognize her integrity and approach her and all her other creations with respect and responsibility. He made extensive efforts to educate government, environmental organizations, educational institutions and Indigenous and nonindigenous peoples: at the local level, all the key environmental organizations affirm his influence to this day. Over the course of my past five years in university, I see an acceleration in attention to environmental issues and Indigenous voice, such as WC commenced negotiating and mediating over fifty years ago. Suffice it to state that the reflexivity earned through my intense outdoors experience on his lake, documented in the photo journal, has contributed to my growing environmental stewardship; as well, it offers a map for others to journey into WC's world. Further, we keep this reflexivity motional, emergent and relational via a Circle of All Nations Nature CAN TEACH Facebook page. But western thinkers have let the analytic rational left brain dominate and lead and have abandoned the intuitive thinking of the right brain. Douglas Cardinal, world renowned Indigenous architect suggests that the academy too is in danger of letting the right brain atrophy; Indigenous peoples access this zone via ritual and ceremony and intense immersion with nature and he stresses that this is the pinnacle of science. With the balance of right and left brain orientations, and detachment from gendered materializations, one becomes the 
fearless warrior capable of effective stewardship of nature. (Personal Conversation, October 23, 2017. (PS Further to WC's presentation, amongst others, the Quebec Environmental Commission denied approval of the Megadump at Danford Lake).

\subsubsection{Beyond Borders}

The most profound cyber technology that undergirds WC's work was his wampum heritage. The youngest belt, as WC described in Tolkienian terms, is known as the Jay Treaty Border Crossing Wampum Belt, it was created between 1793 and 1796 by WC's ancestors, at the time that the Canada/US border was being created. This short wampum belt has a backdrop of purple quahog shell beads, with a center line of white Hudson Bay beads, and four short lines of alternating white and purple beads at each end. 'The commitment was made that my people would be able to cross the border, back and forth, without molestation, to their settlements on either side of this border they were creating', WC stated (Thumbadoo/Commanda 2007 Unpublished Joint Journal). The Jay Treaty Border Crossing Belt of the Algonquins served a historical purpose in Indian border crossing negotiations undertaken by Tuscorora chief Clinton Ricard and the Indian Defense League. WC discussed its promise of border crossing movement without molestation. I was always taken by his use of the word, molestation; to an English literature student, it is such an old fashioned, unusual word; one cannot access it on the online Microsoft Word dictionary. When I Googled it ((http://www.thefreedictionary.com/molestation), I learned that the word refers to sexual assault, disturbing, interfering with, annoying, tormenting, attacking, criticizing, tantalizing, ribbing, teasing, badgering, baiting, worrying, and witch hunting. ${ }^{8}$ When you link the concept of cyber steering in a circular causal relational feedback

\footnotetext{
${ }^{8}$ I also checked dictionaries. However my English Dictionary, only suggests disturbance, annoyance and troublesome. I noted also the word Moloch Molech (Hebrew) referring to the chief god of the Phoenicians and Ammonites whose workshop consisted chiefly of human sacrifices, ordeals by fire, mutilation, etc. (The New Webster Encyclopedic Dictionary of the English Language Including A Dictionary of Synonyms and Twelve Supplementary Reference Sections, Processing and Books, Inc. (1952 - 1971) Winnipeg, Manitoba). WC, as
} 
loop with the idea of molestation, you can begin to grasp a sense of the borderlessness theme of this Wampum messenger. Its agency is held in the placement of artificial Hudson Bay beads on the backdrop of genuine wampum beads. Its intent is to return land to borderlessness, interface, permeability and seamlessness, consistent with the Laws of Nature. WC talked regularly about implications of the inclusion of white artificial Hudson Bay beads on the genuine purple Quahog shell backdrop: the message was that the border would eventually dissolve: 'my ancestors knew that the borders would be temporary' (Thumbadoo/Commanda 2007 Unpublished Joint Journal). He believed that the artificial borders enacted by the colonial beads would eventually disappear.

WC also articulated its intangible message as follows. The purple beads were indicative of the spirit of the land and of the original peoples, and he committed to keeping that reality alive on both sides of the Jay Treaty border. Thus the challenge for him was ensure that the purple Indigenous epistemology and ontology remained rooted in the earth, awaiting the fire that would perform ontogenetically, bringing it back to life, much like lightning fire awakens the blueberry. Recall that WC's great, great grandfather, Luc Antoine Pakinawatik, Stick Hit by Lighting, was born in 1803. His mother was from St Hyacinthe, Quebec, in proximity to the Atlantic seaboard. The people of his parent's generation were the creators of this wampum belt; his granddaughter Manian Commanda taught her son Alonzo, WC's father, about this heritage. The earth knowledge, as evident in the remarkable

noted earlier was an avid student of languages, and he studied dictionaries, French and English ones and rated their qualities (for example, he liked the Larousse dictionary); further, he was very precise in his speech. He intended his audience to understand all these meanings of the word in his deliberated use of that word. An even deeper political message than border crossing was intended. He was fully aware of the demonization of Indigenous peoples with the stories of torture, for example with Father Brebeouf; however, for him, some of these stories did not resonate with the legends of his peoples; also, I also recall Algonquin language teacher Bernice Wagoush McGreggor talk about the predatory and sexually abusive behavior of the priests; today, we know these were all true. Studying Foucault and discourse took me to his discussion of crime and punishment and the public spectacles of the actual quartering of bodies in Europe; only after exposure to the North American cultures that had no word for justice did this get transformed. WC believed multiple boundaries were challenged with molestation, and he was referring to all of them when he talked about the border crossing belt. 
description of John Durant's Best Guide in the World already referenced, came from his father Louizon Commanda, who had kept open the doors of interface with the ancestral knowledge systems. The belt underscores the fundamental spiritual message of Indigenous Peoples about borderlessness. The Elder's people, the Mamininini, the nomads, belong to North America, and as such they retain a sacred connection to the land they are born to. As Elder Commanda puts it, my territory is as the river flows as the bird flies and as the wind blows. This conceptual frame defining relatedness is aligned with the inter-connection ideology of Ginawaydaganuc.

WC's formal borderlessness project commenced in the forties, with the creation of the North American Indian Nations Government by Huron activist, Jules Sioui. In 1953, WC became Supreme Chief of this precursor to the Assembly of First Nations. It was comprised of First Peoples from both sides of the border, Canada and the United States. The wampum responsibility reinforced this commitment into the next century. One notes the play of syntropy in his ancestors' anticipation of future borderlessness. In his public outreach during the last decade of his life, he laminated and shared the millennium Citizen article: It is the Year 2000 and Canada becomes the 51 State of the US. He already saw borders dissolving in political and economic fronts. Today, we are a global society.

WC also presented spatial orientation in his discussion of this wampum belt. Contrary to a possible expectation that he saw the artificial Hudson Bay beads only as the border, the barrier, he used it as a directional emblem. In movement and gesture, he reinforced a strong north south orientation; this was the direction of the flow of movement of the people, south to north, north to south, consistent with a cartographic knowledge that predates the European maps that emerged with exposure to the New World. Even with the gesturing and motions, he was undermining the rigidity of border, and provoking the Mamiwinini compass 
of motion. Thus, WC both addressed the issue of border creation and borderless in his presentations on this wampum belt.

Now, I turn to the 2016 GeoHumanites Publication of the American Association of Geographers; it features a special series of articles on Border Topologies, challenging the geopolitical as organizing construct.

First, I learn that issues in equality, conflict and climate change are causing researchers to interrogate borders; they are now examining borders as topographical entities constituted by techniques and apparatus of bordering and fundamental to the production of such processes via practices that underpin them as dynamic entities (Awan, 2016 and Adkins and Lury, 2012). The discussions move beyond the notion of borders as territory with fixed form locked in space and time by introducing concepts of bordering, borderscapes, surveillance, and Iborders into researching to the production of processes.

Further, researchers also note that the ecological has been missing from border studies (re: species, planetary and environmental structures) and thus now challenge the geopolitical as organizing construct, positing instead, for example, Bremner's "theoretical model and design approach for thinking of border topologies as fluid and relational" (Awan 2016 281), in exposing, in her study, the land/sea binaries performed by architecture on archipelago from the seventies, and proposing instead a fluid, mobile, relational, terra-aqueous aquapelago, theorizing from sea (Bremner, 2016); noting escalating climate change, and deep time planetary processes, Biemann asks "How does a geocentric framing that remains so outrageously indifferent to political domination affect the topology of borders?” (Biemann 2016 373). 
Finally, new mechanisms to address the new understandings of border topographies are emerging via technology, social media, digital visuals, virtual geographies, insurgent regeneration, insurgent citizenry, activism and community engagement (Awal 2016 281). The new research, wherein topological thinking views culture as intensive, and borders are rendered comprehensible through study of deep time of planetary processes, calls for emphasis on a methodological approach that creates units of measure and notions of value and practice relationally, rather than as external metrics (Adkins and Lury 2012).

Global crises in equality, conflict and climate change are leading geographers to interrogate border topologies,

i. moving beyond borders as territories with fixed form lodged in time and space,

ii. awakening to the ecological, and

iii. developing methodologies to research relationally

This reflects a shift from the global geopolitical borders performed since 1492, when Columbus sailed the Ocean Blue. It is also more strongly aligned to Indigenous thinking.

WC's ideological foundation is intimated by the word/prayer perpetually on his lips, Ginawaydaguc - We are All Related. Even before he became carrier of the Border Crossing Wampum Belt, he insisted on the concept of borderlessness as the organizing philosophy of his ontology and epistemology.

At this point, I examine his work in the context of the three lenses presented above, and in the context of this wampum belt.

\subsubsection{Boreal Rendezvous}

So do these reflections have anything of validity to contribute to contemporary geography and to addressing the research question regarding any continued relevance of his discourse to study of environment and related matters? I turn now to a book entitled Rendezvous with the Wild, The Boreal Forest (Raffan, editor, 2004) documenting an initiative launched in support for the conversation of Canada's boreal forest region, by Canadian Parks and Wilderness Society (CPAWS), Canadian Boreal Initiative and David Suzuki Foundation. Topping the list of honorary signees of the CPAWS Boreal Program is William 
Commanda, identified as Honorary Elder. WC engaged with the initiative from the time of its preparatory discussion. In fact, I note from Circle of All Nations records that most of the key environmental organizations in the National Capital Region sprang into life once WC engaged with them. The book commences with an Invocation by him:

I offer a prayer for Mother Earth and for the healing of nations

The struggles in the world are rooted in the land

This is the territory of my ancestors

My people were the Mamiwinini, the Nomads

We had no borders in the past

We respected all of Mother Earth

We did not own her

Our territory is of the land

It is in the air

It is in the waters

We travelled across Turtle Island in our canoes

My ancestors welcomed Cartier to this land from their 100 canoes

The canoes were made by hand, not by the tools of industrialization.

And they carried us far

Then the air and waters and land were pure and could sustain us

Now, the air and waters are poisoned

And the birchbark tree no longer grows tall

So we can no longer make our canoes

Peoples are dying of cancers

Our relationships are poisoned

It is urgent, the need for us to take better care of Mother Earth

We must remember our sacred responsibility to Mother Earth

Only then will our relations with each other heal

Only then will we become a circle of all nations and a culture of peace

Prayer from the launch of the Boreal Rendezvous, June 2003

Rendezvous with the Wild The Boreal Forest (Raffan, J., editor, 2004 13)

This is followed by a mini biography of Elder William Commanda:

Elder William Commanda's range of interests is so broad and deep as to be overwhelming, in that he could as easily be seen as a spokesperson for racial harmony in South Africa, as an advocate for Indigenous Justice in North America. Invariably, however, his prayer is drawn from his profound respect for Mother Earth.

Elder Commanda's ancestors were the Mamiwinini, whose birchbark canoes took them across the vast expanses of Turtle Island following the rivers that connected them with other sacred council fires, guided there by pounding rapids, by water embracing rock in a torrential song of power. The Mamiwinini created a protective web of pathways over the North American continent. The elders' symbolic connection with this spiritual heritage of his peoples is reflected by his world-acclaimed, uniquely stenciled birchbark canoes.

Thus, when he was invited by the Canadian Parks and Wilderness Society to help forge better 
relations with aboriginal peoples throughout the boreal forest, Elder Commanda was moved by the sincerity of the effort and decided at that time to share his teachings of the three Wampum Belts, the sacred documents of the Indigenous Peoples' Turtle Island. His commitment to serve as honorary elder for the Boreal Rendezvous touched work that is closest to his heart - that being the reclamation of a non-adversarial relationship with Mother Earth, respecting her profound complexity, integrity and the inherent logic of her energy of balance and harmony.

In passing on that teaching, he inspired many of us who work hard to protect the environment to seek a deeper relationship with Gaia. Always, when he speaks, when he prays, when he conducts smudges to bring people together in common cause, Elder Commanda reminds us with increasing urgency of an indigenous prophecy that warns:

Only after the last tree has been cut down,

Only after the last river has been poisoned,

Only after the last fish has been caught,

Only then will you find that money cannot be eaten.

(Rendezvous with the Wild The Boreal Forest (Raffan, J., editor, 2004 14)

The editor wanted to conclude the beautiful coffee table photo journal book of the experiences of the Indigenous and non-Indigenous participants with some final words from Elder Commanda. This is the text; it includes reference to his wolves, and affirms the repeated reflections on transformative experiences:

\section{My Prayer for the Boreal Forest}

We sit in front of the fire on this bitterly cold evening in January. We have tossed a white birchbark log into the fire.

I remember that night out there in the snow, seventy-five years ago. It was beautiful and white as I crossed the river. The night air was full of that dangerous penetrating cold, the one that comes when Mother Earth invites you to breathe deeply and enter the timeless sleep of the happy bunting grounds. I forced myself to keep trudging to the forest at the edge of the shores of the river. There, I found a pile of collected dead wood standing up, towering over the snowdrifts, as my father had placed it a season ago when he taught me how to anticipate the needs of future travellers.

I lit my fire and my mind strayed to the four big timber wolves who had found me here on their territory, a year earlier, when I was fourteen. I remember so clearly how frightened my little dog was, how I had to hold bim tight to quieten him. After a long time, the leader of the wolf pack sniffed loudly into the night sky, lay on the snow on bis back and wiggled around, then leapt up and howled as he bounded off into the trees.

We were all one, then. The great mother, our great mother, threw her blanket of provision over us all as she taught us to grow strong and brave and resilient, and respectful of the four elements upon which all life depends. That is how we understood what mother meant. And our great father: I saw him up there in the night sky, with countless stars, including my special star, Ojigkwanong, after which I was named. He 
comforted me with knowledge that I was not alone there at the forest's edge. As our rivers connected us Mamuninini, us nomads, from one sacred island of fire to another, across the great rivers and lakes of Turtle Island, we were also connected intimately to a greater reality.

But, notwithstanding the dangers inherent in translating and communicating concepts, we trusted other great mothers and fathers and gradually our own relationship with Mother Earth was eroded. We failed her in our task as caretakers.

I worry now that the loss is almost irrevocable. I watch this burning birchbark log and I weep because I can no longer find birchbark to build one of those canoes with which my ancestors traversed their world. And as we cut the trees, we erode the soil, and Mother Earth cannot hold the rains, and we suffer floods, and the air fills with contamination.

But spirit is also strong and regenerative. I see the prayers of the new people in my homeland join with mine and aim the arrow of awareness into the masses. In this way, we give voice to the wild things and the wild places and reestablish the lines of allegiance with Mother Earth.

May the winds blow fresh across the boreal forest and drive new life into its heart. Megwetch, Mishomis, that I am part of this prayer. (Rendezvous with the Wild The Boreal Forest (Raffan, J., editor, 2004 184)

WC's connection with CPAWS continues. In November 2016, Circle of All Nations was pleased to present copies of his Learning from a Kindergarten Dropout book and his Good Enough For Two canoe video to their annual fundraiser.

It is interesting that this book/experience became part of an academic research paper regarding canoes: "Thinking outside the gunnels": considering natures and terrains of recreational canoe travel (Grimwood 2011 49-69). The research serves to draw several discursive elements aligned with the WC narrative into an academic examination of relevance to the environment. Grimwood explores the conceptual and theoretical underpinnings of the multiplicities of perspectives embedded in the word nature. He examines poststructural research that expose ideologies of nature that are commodified, colonial, nationalistic and gendered (Baker 2002; Baldwin 2004, Erickson 2008, Haun-moss 2002 in Grimwood 2011). Ethics are exposed as a restrictive, adjudicating or moralizing endeavor suitable only for privileged intellectuals or evangelicals (Proctor 1999 in Grimwood 2011), versus ethics as activity involving personal and social processes of reflection, imagination and 
experimentation to create innovative ways of being (Jickling, 2005 in Grimwood 2011). Colonial narratives and social hierarchies are also implicated in wilderness-nationalism discourses, with Indigenous rights and interests minimized and negated, with histories of nature-subduing and empire-building, fur-trading, tree-cutting, mining, and resource extraction hidden (Hodgins 1988 in Grimwood 2011), and with wilderness ideology disguising the realities of northern exploitation by the south. Emerging institutions like the Boys Scouts reveal the club setting agenda of insiders and outsiders, and the gendered opposition of nature and civilization. The wilderness, the Canadian north, is to be crossed but not lived in; such is this version of Nature.

Research also identifies the production of a particular hegemonic Canadian nationalistic identity with respect to wilderness, wherein imagination is gendered, colonial and perpetuates class distinction (Baker, 2002; Baldwin, 2004, 2009; Erickson, 2008; McDermott, 2004; Newbery, 2003; Shields, 1991). (While this is only tangentially the focus of this thesis research, WC's own history is rich in material for this type of scrutiny. The winter trapping grounds of his own grandfather became an American fishing camp for the elite, and the displaced became the disempowered and servants in their own homes). In tracing the moral terrains of environmental 'justice' to address hidden human discourses on nature, Grimwood finds research now moving to relational approaches to responsibility. This leads him to posit canoe as mechanism for social change. Canoeing functions as an intermediary among contexts of leisure, tourism, outdoor education and recreation, and adventure (Hall \& Page 2006; Plummer 2009), with canoe referencing the real 'natural'. Via biophysical nature, canoe permits intrinsic access to nature as teacher of humility and renewal. Biophilia benefits human health and well being and in turn, people become ambassadors of environmental concern and activism, inclusive of political clout and representation. 
Grimwood notes social hegemony in the outdoors subculture: it values human-powered and non-consumptive canoeing as a benign and authentic way to experience remote and rugged wilderness and contrasts this with the perceived anthropocentric and environmentally degrading activities such as hunting, reliant on motorized and consumptive practices. Canoeing is projected as vehicle for accessing the necessary lessons of place to help decolonize nature and foster improved relations with land, engaging emotions and intellect to develop understanding of place, with challenge, social-group inter-dependence and navigation capacity the beneficial sub-themes. I recall Kogi elders from Columbia saying to WC the worst destroyers of their lands in contemporary times are eco-tourism and religious activities (Kogi Visit 2009).

The Boreal Rendezvous celebrated the integral role of the boreal forest in the Canadian identity and well-being through a series of multi-day canoe trips on different Canadian rivers, with high-profile Aboriginal and non-Aboriginal participants (Raffan 2004); recall that WC engaged with this initiative. Grimwood examines Baldwin's review of the activity:

"Scrutinizing the texts produced during this canoe-conservation campaign and framing "whiteness" as a value system (i.e. not reducible to phenotypic difference [acknowledging visible characteristics of organism] or a monolithic identity [large and unchanging]), Baldwin argued that instead of performing nature as purified and anachronistic wilderness space, canoeists played out a politically innocent form of whiteness that explicitly recognized Indigenous as long-time occupiers of the boreal forest. Although this liberal whiteness might be a necessary step in decolonizing geographies, Baldwin argued that it enables those practicing it to disregard their relation to white privilege, which in this case was embodied by canoeists' mobility in subjective positions. They identified with First Nations on the moral grounds that the boreal forest is threatened; however, the paradox here, according to Baldwin, is that despite good intentions, liberal whiteness can only be forged from a hegemonic position of privilege, one that assumes First Nations peoples occupy an authentic margin" (Grimwood 2011 54).

WC repeatedly asserted an earth-grounded right to occupy the centre. Thus it becomes apparent that a diversity of natures is enacted relative to different spatial representations of 
recreational canoe travel. There is a localized enactment of nature in canoeing and nature is played out as

- $\quad$ redemptive or nourishing space associated with human and non-human evolution;

- timeless space that may facilitate spiritual renewal or oppress the Others by making invisible living histories within a landscape;

- located space for intellectual and emotional contemplation;

- emergent space encountered through movement, skill and stories of place-making;

- social space that becomes shaped by hierarchies and influences of power

Social theory contests value free science, in view of recognition of socially constructed knowledge. Dualistic traditions, attributable to Cartesian impulses of human exceptionalism (Haraway 2008) are reflected by an assortment of binary pairings that appear to order the world by an assortment of pairings, for example, mind/body, self/other, object/subject or human/animal (Haraway 1991). In nature/culture debates, culture succumbs to what is determined to be natural law or, the reverse, societal advancement results in atrocities upon nature. Both positions, however, function as two sides of the same coin whereby those things considered natural are rendered mute or inactive and culture is the assumed domain of agency (Hinchliffe 2007). Objectivist epistemology is at the core of human/nature dualism, and by extension, the contemporary environmental crisis (Bai 2004; Haraway 1991).

Grimwood suggests interventions can be made to enact nature differently, and this developed further, subject to descriptive empirical studies, which could provide richer data than prescriptive approaches. Geography has been increasing aligned with the ethics movement and he questions whether a moral geography might be derived via thick (detailed) descriptive ethics. Further, geographers are contributing to a relational environmental ethics. Braun (2008) acknowledged a marked shift from "dualistic" to "relational" ontologies within geographic studies of society-nature relations; this announcement matches growing attention 
to relational thinking in broader contexts of human and cultural geography (Castree 2003b; Massey, Allen, \& Sarre 1999b).

\subsubsection{Canoe as Epistemological, Ontological and Ontogenetic Tool}

I turn from Grimwood's "Thinking outside the gunnels": considering natures and terrains of recreational canoe travel to William Commanda's canoe project - Canoe as epistemological, ontological and ontogenetic tool. As noted earlier, canoe spirit was inherent to WC's blood line, and as a toddler, his mother, descendent the line of canoe artists of Lac Simon/ Chiman, Quebec, taught him how to make tiny craft canoes, and sail them in the gullies eroded by snow melt, or spring rains, or thundershowers. His study of water, colour, vacuity, opacity, turbulence, movement, current, velocity, force, sound, and nature craft creation commenced early. Never idle, by the time he was eight, he had learned to study canoemaking seriously with her brother, Uncle Andre Cayer. One of his memories of this school was of him ruining his uncle's crooked knife and being chased away; but he already knew intuitively that canoe was not only his blood-line but also his life-line, so he hung around till his uncle relented and let him back into the shop/school. Note that while all the Algonquins built the iconic birch bark canoe, this likely being their most important material artifact, Uncle Andre came of the line of shaman/artist, and so his skills were particular. Making and selling canoes for tourists in the nineteen twenties gave William a taste for independence and autonomy early in life. This also taught him to be alert and astute, as commodification of art became critical to survival in the depression of the thirties. He told me how he sold a canoe for $\$ 15$ to the middleman in Maniwaki shopkeeper, X. A few hours later, at the gas station, his saw the fifteen-footer on the car of an American tourist. He asked how much the man had paid for it, and learned it had been sold for much more. WC also informed me that he was not paid in actual money, but rather in goods from the store itself, and he was not 
unaware of this abuse. Ever the diplomat and multi-focal thinker, he also noted that perhaps that was as well; there were some benefits to the transaction: he had food where he might have spent the money on liquor. Further this shop was also the only place folk congregated in the racist town populated by Irish, Scottish, English, French, American tourists and Algonquin. This manner of acquiescence was also a curious expression of subjectivity and personal power in the acknowledgement of the colonial control. But he also knew how his people were being abused by greed and control of the colonizers. Decades later, he operated his own craft shop, and his sign was in English. One day a representative of the provincial government came to inform him that he would have to make his sign French; WC's response was And who is going to make me, you and who else? (Thumbadoo/Commanda Unpublished Joint Journal 2007) Yes, there were multiple lessons from the early canoe school education. Later, he and his wife Mary, herself a skilled craftswoman, taught their grandchildren canoe-making and independence, sharing the resources from sales equally, and perfecting the canoe stencil etching that identifies most Commanda canoes from that period. It took extra effort to secure winter bark for the etching, and the nature motifs constitute a WC signature as artist. David Gidmark wrote a book on The Indian Crafts of William and Mary Commanda (Gidmark 1967).

Later, in the eighties William's canoe academy went mobile: he built canoes at Expo 67 in Montreal, and at the Canadian National Exhibition in Toronto; he conducted canoe workshops on Victoria Island for the National Capital Commission; in 1981, he and his wife built a canoe for Queen Margarethe of Denmark, housed in the Roskilde Viking Ship Museum, and two books on him were created in Danish (he considered this venture reconciliation with the Vikings after their act of blood-shedding, when they had first arrived in North America, over a thousand years ago, and killed the Beothuk; note that this links 
with the agentive message of the Seven Fires Prophecy referenced in the 1987 Aboriginal Constitutional Conference); he presented a canoe to Haida artist Bill Reid, and received one of his paddles; the seventy odd canoes he built over the course of his life time went to countries all over the globe. In 2000, WC offered the opening greeting at UNESCO's World Summit on Arts and Culture, but he brought environmental teachings into the event, as noted in Learning from a Kindergarten Dropout: "in his prayer was woven his concern for Mother Earth; the poignant message was that because the resources of Turtle Island were depleted, the waters contaminated, and the air polluted, the birch tree no longer grew tall and big, nor the cedar tall and straight, and he could no longer practice his art and craft in the land that was hosting the conference" (Thumbadoo 2005 32). This is also deeply distressing from an ecological perspective because the first plants noted in Beringia twelve to fifteen thousand years ago were small birch trees. He conducted the opening ceremony for the launch of the Canadian Canoe Museum, founded by his friend Kirk Wipper in Peterborough, a millennium initiative of then Deputy Prime Minister Late Herb Grey. The museum hosts a special display of his canoes. WC blessed the waters brought in from each province and territory in Canada, reminding people that "Rivers are the Veins of Mother Earth, that everywhere her flow is blocked by dams, and that her waters are perilously contaminated" (Commanda Archives). In 2003, at the age of 90, WC's canoe academy entered the cyber world, with the creation of a video, Good Enough for Two, by Valerie Pouyanne and a French videography student, Maureen Bartholomeus, when he shared his canoe-making skills with Mi'q Maq craftsman Todd Labrador. Since then, birch bark canoe making has become a vibrant presence and reassertion of Algonquin in Canada. In 2007, it was declared number one on CBC's list of the seven natural wonders of Canada. In 2017, with the launch of the Museum of History's Canadian History Hall, a birch bark canoe created by WC's student Todd 
Labrador took place of prominence. His grandson Chuck Commanda has returned to the craft, creating canoes and birch bark baskets with the students, Plenty Canada, Canadian Canoe Museum and the Museum of the Native American in Washington DC. Marcel Labelle and Pinook Smith have delivered canoe workshops at the Universities of Ottawa and Carleton, and elsewhere, as the Indigenous heritage enters the academy.

WC also launched Paddle for Peace events at Victoria Island to honour the Fall equinox, celebrate the birchbark canoe heritage, acknowledge the United Nations International Day for Peace, and animate his vision for the Asinabka Sacred Chaudiere Site and the Indigenous Center and Eco-Peace Building Think Tank schools he envisioned for all. He demonstrated in action and influence the critical connection of physical and human geography in the now desperate global search for environmental equilibrium and peace.

Canoe has meant all of this and much more in the curricula of WC's Circle of All Nations 'school', now launching into social media. A recent post on Facebook drew this storytelling stream of thought to mind, and within a day it had reached hundreds of people; this perhaps affirms the value of detailed thick description not only for qualitative research and critical reflexivity, but also in teaching relationality:

The Gathering of Canoes promotes healing, reconciliation, and respect for Indigenous host nations 230 people reached

Circle of All Nations shared your post.

July 14, 2017 at 8:56am

Canoe Spirit re-colonizing the country

Romola Vasantha Thumbadoo's Face Book Page

Here's a bit of history that is now 21 years old - It was the Native Friendship Centre of Brant that first inspired this outcome - It was a project I supported when I worked in Urban Aboriginal Policing - Under the leadership of Trudi, the Centre brought Aboriginal youth, parolees, inmates from the provincial jail and Brantford City Police together to participate in this cross cultural sharing learning initiative called Fast W aters Fast Friends in 1996: one weekend they met to learn about the project, they next they paired up and the Grand River tanght them how to support each other. Then all the community got together for a community feast; there were over 200 people at the feast-yes in 
Brantford! Trudi was so enthusiastic about the initiative that cops were lining up to participate. The event was video-taped. We profiled it at the first Urban Aboriginal Policing Forum in Winnipeg in 1996 with a special presentation by Trudi. Canadian Association of Chiefs of Police was a partner with Sol Gen (now Public Security); thereafter Craig Nyrifa of the Saskatoon Police developed a twoweek canoe cross-cultural camp experience for youth at risk and elders. RCMP took this idea to BC and it became the Pulling Together initiative - Ed Hill was involved; Kirk Wipper Founder of the Canoe Museum joined them and came to report about this at a Circle of All Nations Gathering years later! With Grandfather, master birch bark canoe builder, water steward man and peace builder, we developed the Paddle for Peace initiative and acknowledged the Fall Equinox with this canoe bridge building paddle event designed to line up with the UN International Day of Peace initiative, and our environment/clean the river advocacy, because we knew environmental and peace issues were inextricably linked. And recently, Max Finkelstein reconnected with us regarding the Paddle Brigade he was coordinating, destined for Victoria Island. While Circle of All Nations has not been in a position to continue with Paddle of Peace events at Victoria Island as per Grandfather Commanda's initiation, still his Canoe Spirit reigns. This May, I went to a special screening of the marvellous canoe Todd Labrador created for the new History Hall at the Canadian History Museum - he is part of our/V alerie Pouyanne's GOOD ENOUGH FOR TWO Canoe Documentary with Grandfather William Commanda. A week ago Larry McDermott of Plenty Canada, a partner in the Paddle for Peace events, hosted a reconciliation workshop and Grandfather's grandson Chuck Commanda commenced a birch bark canoe-making workshop there! And I just saw a post of Marcel Labelle doing canoe work in BC - be created a mini birch back canoe (the Knowledge Canoe) for Grandfather when Willis College and Technowave, Rima Aristocrat and Cliff Summers presented a scholarship in bis name. Well, Grandfather built over 70 canoes including the one be build for Queen Margerethe of Denmark - and these went to locations across the world - Great to see the CANOE SPIRIT continuing its Work Bring People Together with Nature in the Spirit of Friendship. Note the photo I shall post below - it shows the Dreamcatcher created by the Friendship Center of Brant youth and gifted to me in 1996 - it hangs at my window still guiding my dreams!!!

Relational thinking jettisons either/or categories, recognizing instead "the important elements of interconnection which go into the construction of an identity" (Massey, Allen \& Satre 1999a 12). Rather than a world made up of purified forms that frequently mix, for example, between pristine wholeness and human civilization, relational thinking envisions relationships as the primary "unit" of reality (Bai 2004; Haraway 2008). An entanglement of human and non-human relations that are at once conceptual, ideological, material, discursive, practical, dynamic and political blur the boundaries between presumed substantive entities. It is a shift from an ontology of division and determinism to an ontology of radical uncertainty, configuration, mobilization, hybridity and impermanent relations that forms durable bodies 
(Hinchliffe 2007); the canoe epitomizes this motional cartography in a most profound manner.

It is the journey motif that influenced the integrated ontology, ontogenesis and epistemology of WC. The opening pages of Learning from a Kindergarten Dropout (Thumbadoo

2005 13-14) draw attention to the cosmic and the Canoe moon:

The Father asked him where this God (i.e. Manitou/Creator) was before he created the earth. "In bis canoe" be replied "which was floating upon the waters.

It is one simple line pointing to a creation story of the Mamiwinini. Yet interwoven in that motif is the spirit of canoe that connects the ninety-year old elder to his Creator and his earliest ancestors and, battered perhaps but still floating, drives him into the future with tireless grace and determination.

When I first met him, William Commanda told me about how more than a hundred canoes had greeted Cartier when he arrived in North America, indisputable proof that his people had been on the continent from time immemorial. A copy of a painting of a couple of dozen birch bark canoes on the waters of Abitibi Lake in Northern Quebec is pinned on the door leading down to his basement, alongside the now famous photograph of one of William's own canoes.

One line of his story can be traced in the passage of the canoe through the years... Even today, the canoe is perhaps the central image to associate with William Commanda. He is a master birch bark canoe maker. He learned how to build and perfect his canoes as a child; he earned his independence through them; and his creative spirit is marked on them. He preferred to use winter bark for his canoes, which was more difficult to secure, just so that he could imprint symbols of the world around him on them - today, these images of stars and moose and beaver and bear constitute the unique signature of the artist on his canoes. In 2004, at the age of ninety, he built his latest canoe; this experience is captured in documentary by Valerie Pouyanne entitled “Good Enough for Two!” (Thumbadoo 2005 13).

This canoe school theme captured in the documentary lends itself to further analysis in postdoctoral research.

\subsection{Concluding Remarks}

It is common knowledge that most Indigenous peoples are highly suspicious of researchers, and WC himself was extremely circumspect about whatever he put his signature to. I have discussed my kayak school experience in the context of a nature-based photo- 
journal imaginative. Here I present audio and visual clips of WC, and an audio recounting of my engagement in WC's discourse in an internet radio interview with Prophecy Keeperz, to demonstrate the iterative, reflexive emergent components of our joint research project. Inclusion of this material is demonstrative of our animation of non-textual creativity and orality in the reflective western frame of our conceptual Circle of All Nations medicine wheel of learning. In this sense, the hesitations, reflections, fluidity and iterations of the oral are demonstrable signifiers that illustrate "complex constellations of ideas and thoughts about research in more simplified categories of knowledge" (Mansvelt and Berg in Hays 2010 343). Reports on WC's work focused directly on environmental priorities are available on our websites. They attest to his reach and influence.

\subsection{Summary of Findings}

The attached video and audio files complement the textual presentation with the critical reflections of both WC and myself.

1. At 90, William Commanda decides to build another canoe to teach his craft to Todd Labrador - Good Enough for Two documentary by Valerie Pouyanne, Loon Productions.

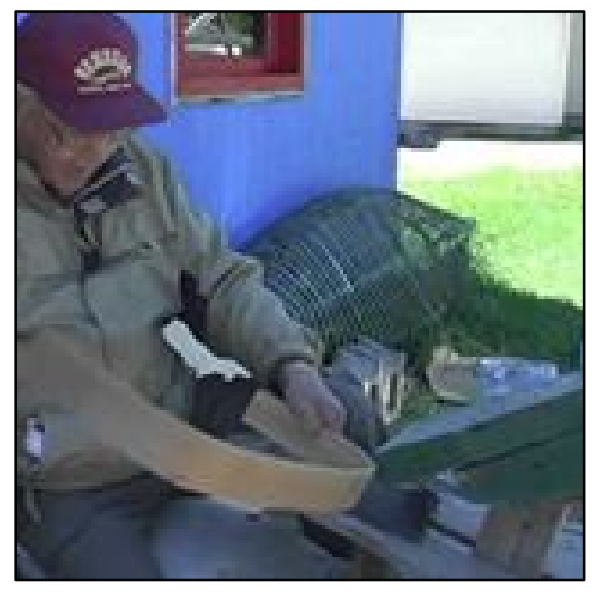

Figure 17. Good Enough for Two Documentary (Valerie Pouyanne, Loon Productions, https://www.youtube.com/watch?v=JArzmp9pQPI) 
2. Audio interview with Prophecy-keeperz, demonstrating reflexivity and storytelling Romola V. Thumbadoo

https://soundcloud.com/mackboogaloo/prophecy-keeperz-romola-thumbadoo-ep60-thelegacy-of-william-commanda

3. The following link was posted (October 18, 2017 by the young nephew of Donald Marshall Junior, (who came from Nova Scotia to participate in the Circle of All Nations gatherings of William Commanda); his Facebook post demonstrates the reflexivity and impact of the grassroots Indigenous environmental school and movement both Indigenous leaders inspired. It captures the essence of the William Commanda Lament.

https://www.Facebook.com/ScienceNatureUniverse/videos/1501033016652147/

\subsection{A William Commanda Phenomenological Exemplar}

An Example of a William Commanda Reflexive Intervention in the plight of the American

Eel, and its Trail of Influence

In 2007, William Commanda intervened in the federal Species at Risk consultation on the American Eel. These were his remarks:

EMAILED MESSAGE RE CONSULTATION on American Eel March 31, 2007

William Commanda Intervention in Consultations on the American Eel as a Species at Risk Department of Fisheries and Oceans Sent March 31, 2007 Copied to Bill Allen, Archaeologist, Alan Latourelle, Parks Canada, Meredith Brown, River Keepers, and Larry Graham, Ottawa Heritage River Designation (noted, RVT Feb 7, 2014)

Dear Pooi-Leng Wong:

I wish to add my name to the list of people petitioning for the designation of the American Eel as a Species at Risk.

I am a 93 year old Algonquin of the Ottawa River Watershed, and I currently also serve as Honorary Chair on the Ottawa River Heritage Designation Committee. I have been deeply concerned with the devastating transformation of this watershed and areas beyond throughout the course of my lifetime, and I have been increasingly actively engaged in efforts to promote respect for Mother Earth and all that inhabit her, over the past twenty years. This effort was in part recognized by the Canadian Heritage Rivers System, when, in 2004, I was presented with the Bill Mason Conservation Award. 
Over the past ten years, I have also been involved in developing an integrated vision for an international environmentally focused healing and peace building centre at the sacred meeting place of my ancestors, Asinabka, site of the Chaudière Falls in Ottawa, a centre to advance respect for Mother Earth, Indigenous Peoples and all others, and in my mind, these are interrelated matters of grave concern for the world. Last November, I organized a Water Life Workshop, in part to advance this effort, but also to encourage better stewardship of the Ottawa River. This led me to an effort to dissuade Domtar Inc. from expanding the Hydro Electric Plant at the Chaudière Falls. Amongst other concerns, I have been upset by evidence of the destructive impact of turbines on eels in some graphic photographs.

I believe it is crucial importance that we all reexamine our invasive and destructive relationship with Mother Earth and all her creatures, and many are beginning to hear this cry. Every day, it seems we hear of the desperate state of yet another species, and the current focus on sharks highlights the interrelated ramifications of the destruction of individual species.

At this point I am raising my voice concerning the plight of the American Eel. The eel has been of spiritual, nutritional and material importance to the Indigenous Peoples of the eastern seaboard, and to my ancestors of the Ottawa River Watershed, since time immemorial, has played a role in the Donald Marshall Junior struggle for the recognition of indigenous fishing rights, and I believe eel spirit is intrinsic to the sacred Seven Fire Prophecy $W$ ampum Belt that I have carried for the people for over thirty six years. It is this prophecy that tells us that humanity is now at a cross roads, and that we need to regenerate our relationship with Mother Earth and each other, and it is a message I delivered at the December 2006 Minster's Round Table on Species at Risk; a short few months later, we all alert to the issues of global environmental and human crisis.

I am taking the liberty of attaching a paper prepared by Archeologist Bill Allen, who is currently undertaking studies in support of my effort to advance a vision for the Chaudière site, for a more specific review of critical concerns on this matter.

I look forward to being apprised of development on this file.

Sincerely, William Commanda Algonquin Elder www.circleofallnations.ca

An updated comprehensive Circle of All Nations report on the American Eel is available at www.Asinabka.com, inclusive of engagement in a second round of federal consultations. Circle of All Nations colleague, Indigenous justice and rights activist Donald Marshall Junior, joined in William Commanda's advocacy efforts, aligning his leadership with fight to protect the ancient nomadic fish of their ancestors, that transformed as it travelled. Exactly a year after William Commanda's death, seven eels were found at Victoria Island, indicative to some of animal agency. The effectiveness of his teachings can be observed in heightened awareness of the plight of this amazing creature today in the National Capital Region. In response to his challenge to dam renewal at the Sacred Chaudiere Site, while on the one hand, the dam has actually been expanded contrary to his wishes, media informs that eel ladders have been installed, baby eels have been released into the downstream waters, and environmental and community groups are now engaged in activism to protect the species, one such effort being announced at the Circle of All Nations October 2017 Peace event. While 
the optics and effectiveness of these interventions remain under serious question, certainly eel spirit is alive.

The following clip demonstrates WC's strategic storytelling approach.

http://www.asinabka.com/MP3s/William\%20Commanda $\% 20 \mathrm{Re} \% 20 \mathrm{Land}, \% 20 \mathrm{Eels}, \% 20 \mathrm{Pr}$ ophecy, $\% 20$ Indig $\% 20$ Centre.mp3 


\section{Chapter 7}

\section{Theoretical Lens: Cybercartographic Geo-Narrative Encore Vivant and Cyber Steering - Potential and Volition inspired by the Missing Sacred Wampum Belt}

\subsection{Orientation into the Cybercartographic Geo-Narrative Logic of Inquiry Lens}

This chapter examines the WC cyber geo-narrative in the context of borderlessness and movement and travel, the organization or participation in conferences or events, and evolving digital technologies, websites, blogs, and social media communications. The purpose is to document and analyze the presence and influence of WC's voice on environmental and related issues from a contemporary perspective. This modern expression of performance mapping will draw the cyclical research project to the fourth interpretative lens, and the emergent zone in the conceptual Circle of All Nations medicine-wheel-in-motion spiral.

In addition to his own organization of conferences and events to share his messages, WC travelled globally extensively throughout his life, and with growing urgency towards the end of his life, to register his concerns and warnings about the looming environmental crisis. Social media has also been used extensively to animate the Commanda discourse. From the time of the creation of his first website in 2000 and on-line blog posts, WC has been known as the techno elder, and well before his death, he was part of the emerging Facebook world. Since his death, and in the absence of the continuation of his land-based gatherings to build relationship and community, Circle of All Nations maintains several Facebook pages to inform the public of his environmental and peace building passions. It also host regular events aligned with cosmic cycles of the solstices and equinoxes, focused on his environmental and 
peace-building priorities. These activities are designed to reach a diversity of people, attract new people, profile co-created efforts, and articulate the relational priorities and potential of the Circle of All Nations.

The agency of the Missing Wampum Belt guides some of the analysis. In a shadowy way, it continues to influence and support the WC work. I explore also the potential of both cybercartography (reflecting the interplay of time and space, grounded in the land, physical and sensual data, and multiple perspectives of a range of key participants accessed in textual and photo/video/audio) and Facebook/social media to both advance and analyse his work.

The digital technologies reveal and track a continual process of cohesion and coherence, disruption and rupture, and emergence and transformation. The cybercartographic tools explore the presence and agency of a diversity of players in both archival text and audio-visual material, and the inclusion of the multiple voices/players/material provides a rich database. The technologies also track the on-going and emergent discourse. I probe the role of chance, a motif somewhat like the energy of the trickster, in the generally uneven interplay of entropy, syntropy and negentropy, which over time, can reveal rhythm and pattern. This can be scrutinized in the digital tracking technologies, for example the likes or hits in Facebook. Examination from the Actor Network Theory (actors and actants) and praxis angles augments understanding of the relative power, agency and activism of discursive elements, but unfortunately detailed analysis, however promising, is beyond the scope of the thesis research.

I use the cybercartographic atlas as a research method to examine the earlier and the contemporary WC/Circle of All Nations discourse. This is to some extent a digital technology test of the continued relevance of WC's approach to environmental stewardship. At the same time, I argue that the cybercartographic atlas as well as other digital and social media 
serve as a part of the on-going discourse. WC's Facebook pages, which can generate thousands of hits in a few hours, affirm preliminary conclusions about the continued on-line interest in him. This provides some evidentiary support for conclusions about the importance of his years of work and outreach, legacy and the role of praxis in cyber activism. For the purposes of the thesis research, only the segment of the Commanda discourse pertaining to the intangible relationship to sacred land is examined. More time and scrutiny is required for analysis of the vast body of archival materials accumulated over the course of the past century.

Research findings can lead to the negotiation of new spaces and times for diverse and random intersection, and can spark and ignite of new thinking, intersectionality and relationality.

\subsection{Theoretical Frame, Methodology and Tools: Cybercartography, Auto- Ethnography and Critical Reflexivity}

The cybercartographic atlas and social media constitute the research laboratory and

illustrative method to examine the geo-narrative, processual and performative mapping nature of WC's Circle of All Nations work and reach. The medium's graphic, sensual capacity, complemented by Facebook animation, permits a critical examination of the emergent potential of this particular legacy. This penchant is consistent with WC's own style. His archives hold scrapbooks of newspaper articles going back fifty years. He had slides and a slide projector from the earliest times of their availability on the market. He used cameras and video cameras over the decades. Ten years ago, he acquired the very first IPhone. Unfortunately, an eye injury limited his engagement with the computer. In the introduction to The History of Cartography, Volume Two, Book Three, Woodward and Lewis note in Volume One of the series, that maps had been defined as "graphic representations that facilitate a 
spatial understanding of things, concepts, conditions, processes, or events in the human world" (Woodward and Lewis 1998 1). However, following the ground shifting ideas of Harley in Deconstructing the Map, (Harley 1989), it became apparent that this Eurocentric notion failed to or could not incorporate the special understanding of the peoples of traditional societies. WC and I travelled many times over large expanses of the American North East, and his memory of rocks, streams or turns in the roads after thirty five years was uncanny. The memories that the journey over the land generated were also multi-layered, pentimento fashion; and it took much attentiveness and repeated exposure for me to acquire some small comprehension of WC's experiential mapping knowledge.

With spatial technology comes the integration of visualization, knowledge discovery in databases and distributability via Internet. Distributed maps unlike static maps link information from various sources within a user-defined environment. The information may be present in many forms (maps, images, sounds, video and text) in a multimedia database, and its use demands interactivity. These online spaces are cyber spaces for a new kind of mapping. I suggest that they incorporate multi level processual and performative components comparable to Indigenous mapping approaches.

Geo-narrative and cybercartography are a hybridized approach to the critical scrutiny of the contemporary relevance of the legacy of Indigenous Elder William Commanda. Does the field offer opportunities to reconcile past and future by being active in the present, bringing new knowledge to physical and human geography and to mapping multiple stories? I believe the proposed method offers innovative interpretative research material for this thesis. Further, it lends itself to a deepened exploration of syntropy.

Telecommunications map technologies such as Google maps are highly interactive, and engage the user in innovative ways in mapping; they are being applied to an exploding 
range of topics of interest to society, and not only to location finding and the physical environment; this digital mapping technology is not a stand-alone product like the traditional map but part of an information/analytical package. This type of mapping is compiled by teams of individuals from different disciplines; and it involves new research partnerships and the private sector (Taylor and Pyne 2009 3); and social media is making it accessible to the public at large.

More than a decade ago, D. R. Fraser Taylor, Director of the Geomatics Cartographic Research Centre (GCRC) at the Carleton University, the academic pioneer in the field of cybercartography, wrote that this technology would see cartography applied to a much wider range of topics than had traditionally been the case: "It will also utilize an increasing range of emerging media, forms and telecommunication networks such as the Internet and the World Wide Web. It will be a multidimensional cartography using multimedia formats and is more likely to be an integral part of an information package than a stand-alone product. Cybercartography will also be highly interactive and engage the user in new ways. In organizational terms, it will see new partnerships being created between national mapping organizations, the private sector and educational institutions and the products of cybercartography are likely to be compiled by teams of individuals from very different disciplines and professional perspectives working together" (Taylor and Pyne 2009). Taylor and Pyne and several other researchers discuss the evolution of cybercartography in instructive and ground-opening ways. It involves intense interaction between theory, technology and practice; it integrates quantitative and qualitative information in new ways; and its main product, the cybercartographic atlas is a metaphor for a new form of organization, analysis and presentation of a wide range of material referenced by location. In the work of the GCRC, the tiered information framework was named the Nunaliit 
Cybercartographic Atlas Framework in 2006, reflecting in its identity the Indigenous community partnership in its evolution. The community-based approach drove the development of the software in different domains: open specification approaches; modularity; live data; geospatial storytelling; and audio-visual mapping (Caquard et al 2008). It also permitted the holding of geospatial information created by multiple participatory mapping activities in a dynamic and emergent structure (Pearce 2008 21). The implementation of the Lake Huron Treaty Atlas supported the potential of geonarrative to reflect personal experience, multiple perspectives, as well as the inter-scalar relationships between individuals and institutions (Pyne 2012).

Critical cartographers have exposed hidden and sometimes hideous narratives and metanarratives and agendas embedded in maps, particularly colonial and imperial maps. Thus, maps tell stories and the stories maps tell both reflect and create reality. Caquard explores the potential for hybridization: between cartography and creative disciplines; between the grid map and the story map; between fiction and reality; between map and the territory. Of interest is his assertion that, more and more, maps are used as an analytical tool to explore the spatial dimension of narratives, marking the emergence of hybridized practices such as literary cartography.

Finally, I turn to "Maps, Narratives, and Trails: Performativity, Hodology, and Distributed Knowledges in Complex Adaptive Systems: An Approach to Emergent Mapping”, by David Turnbull (2007). This article is rich in its exploration of means to explore emergent mapping. My hypothesis is that William Commanda's Circle of All Nations work is processual, performative, emergent mapping, and that the cybercartographic atlas is an extremely engaging platform to study its diverse and evolving characteristics across time and space. In 2014, Developments in the Theory and Practice of Cybercartography, (Taylor, ed., 
Lauriault, Assoc. Ed. 2014) was published, inclusive of much new material in the field; the general discussion that follows examines articles in this publication. The subtitle, Applications and Indigenous Mapping is also an affirmation of the statement that "(t)his volume is based largely on an entirely new practice developed since 2007 in cooperation with indigenous groups in Canada" (Taylor 2014 4). In the context of this research focus, it is interesting that William Commanda offered a blessing at the launch of the Lake Huron Treaty Atlas at the Geomatics and Cartographic Research Centre, Carleton University, in 2007; and that exposure to the potential reach of the technology inspired his own academic interests.

In the discussion of new practice, reference is made here to traditional knowledge as "an aspect of ecological management and environmental stewardship, sustainable development economic empowerment, self determination, human rights, culture, arts, craft, music, songs, dance and diverse creative repertoire: religion, lifestyles and innumerable aspects of social processes that undergird a people's worldview" (Oguamama 201146 in Taylor 2014 5). These matters were to some significant extent a part of William Commanda's performative approach to the environment and relationships.

Pyne notes that critical cartographers recognize the significant ways maps are social constructions, have excluded certain perspectives, how such exclusion has been systemically guided by imperialistic and colonial goals and how this can be remedied by viewing maps as processes and through use of alternative mapping approaches (Pyne in Taylor 2014 252, 253); and she notes the nonlinear, non-teleological and spatialized approach to the design of the Lake Huron Treaty Atlas involved a revolving series of iterative processes along different dimensions. This iterative process can reflect change and transformation that goes beyond online web-based instantiation through inclusion of variety of interactions and experiences occurring over varied extents of time and giving rise to emergent knowledge (Pyne in Taylor 
2014 246). Pyne also examines the relevance of the epiphanic processes in iterative design, epiphanies being the sudden and abrupt insights and or changes in perspectives that transform the individual's concept of self and identity through the creation of new meaning and transcendence; this is relevant to approaching cartography performatively and to the mapping experience itself (Pyne in Taylor 2014 255, 257).

Reference is also made to Howard Gardner Multiple Intelligence Theory (Taylor et al), where Gardner argues that we learn in very different ways, and he describes eight intelligences: linguistic, logical/mathematical, musical, bodily kinesthetic, spatial, interpersonal, intrapersonal and naturalist; there appear to be similarities in this approach to the central concepts of cybercartography (Taylor 2014 299, 300); this theory also offers parameters for examining learning and teaching in the context of WC's range in knowledge and knowledge generation within a dynamic and diverse community context.

Partially grounded as it is in an Indigenous foundational thinking, cybercartography represents a critical, fascinating, evolving, contemporary, spatial and temporal means to research the multiple layers of the WC land-based history, personal story and living legacy, through his personal narrative and through past, present and future iterations by the multiple animators of his Circle of All Nations global eco-community.

Cybercartography presents tools to explore the Indigenous mapping experience as a multi-dimensional/multi-leveled/multi-pronged, vibrating, cognitive and sensory interface, much more closely approximating the rhythms and rules of nature, as incorporated in the Indigenous animation of mapping. I come to the use of the cyber map, grounded in its technological and science based foundations, and emergent from cognitive principles, to explore the multiply constituted, ontogenetic, performative, social discourse of William Commanda, emergent from his Law of Nature Indigenous legacy, grounded itself in in-body 
motion/cognition/generative principles. Modern cartography and cybercartography bring me to a spatial and temporal understanding of Indigenous cartography in a manner more attuned to it than European mapping models. As such, they serve as a key qualitative research methodological tool to analyse the contemporary significance of the legacy of William Commanda, through the inclusion of photographs, audio and video materials, text and art and reflections from WC's archives and contemporary refection, on a digital map platform.

The importance of narrative/storytelling/oral discourse to Indigenous peoples impacted its development, causing the shift from a publishing/presentation model to a participation/processual model, and permitting multiple narratives (Crampton 2001). Cybercartography permits the expression of the "geographies of human experience and place on the map" (Pearce 2008 17), "gives experience its own layer" (Pearce 2008 21) and maps "personal trajectories of experience across the digital map using GPS". (Pearce 2008 21). It also permits the holding of geospatial information created by multiple participatory mapping activities in a dynamic and emergent structure. Caquard (Caquard, 2011) examines the relationship between maps, narratives and metanarratives in Cartography 1: Mapping narrative cartography. He identifies story maps, fictional cartography, narrative atlas, and geospatial storytelling as characterizing the growing interest in the relationship between maps and narratives. Turnbull acknowledges Indigenous in his abstract: "If maps are conceived as representations of reality or as spatially referenced data assemblages, a dilemma is raised by the nature of Indigenous knowledge traditions and multiple ontologies" (Turnbull 2007 140). Here, he discusses the issue of resolution of the incommensurability of multiple ontologies and knowledge bases in conjunction with performativity and hodology (a term he resurrects in view of its rootedness in trails) in creating databases of distributed knowledge - that is, 
"Through focusing on the encounters, tensions and cooperation between traditions and utilizing the concept of cognitive trails - the creation of knowledge by movement through the natural and intellectual environment - the socially distributed performative dimensions of differing modes of spatially organized knowledges can then be held in dialogical tension that enables emergent mapping" (Turnbull 2007 140). Circle of All Nations has expressed this as you/we make the road by walking it.

Particular uses of cybercartographic techniques demonstrate ways to examine the Commanda archives. For example, we learn from Payne, Hayes and Ellison that in "photobased conversations between elders and youth and through the cybercartographic site, archival photographs are being used... to link past and present ... and strengthen ... cultural heritage (T)hrough its intergenerational collaborative, interactive, and interdisciplinary format ...” (2014 199); place naming ensures Indigenous language promotion, preservation and transmission (2014 226). Keith, Crockatt and Hayes suggest that place naming and story/legend sharing also open up understanding of special/sacred place, redefining geographical landscape in across space and time from First People viewpoints (2014 234); and Aporta et al find in their research that illustrating the complex knowledge bases of oral literacy and storytelling with the cybercartographic technologies allows for the dynamic combination of data from different sources and of different types, (audio, video and photographs) on a map (Taylor 2014 239, 242). Materials and information generated via the new technologies can be shared broadly; what is also interesting in the various research projects is the desire of the Indigenous participants to share this Indigenous knowledge more broadly with scientists and public (Ljubicic et al in Taylor 2014 203), because they are such an acknowledged and visible part of it. Relationality, consultation and verification contribute to the authenticity of the research. (Developments in the Theory and Practice of 
Cybercartography, (Taylor, ed., Lauriault, Assoc. Ed., 2014).

\section{Methodological Tools}

The cybercartographic atlas, social media, and post-2011 activities permit a new means to scrutinize any ongoing relevance of the Commanda approach to environment and related matters, from

a) land based language and symbolic imagery (petroglyphs, pictographs, canoe motifs), to

b) William Commanda's use of the Circle of All Nations as a zone of encounter to address incommensurability, to

c) photographic reflection on the intangible, compelling voice of space and place, to

d) contemporary social media engagement with William Commanda and his ideas.

One approach involves a time-based lens scrutinizing his discourse over the course of his 98 years, and then its ongoing presence since his death in 2011, primarily on one particular aspect of his discourse, his assertion of intangible, sacred relationship with land in his Circle of All Nations Asinabka Sacred Chaudiere Site legacy vision.

\subsection{Conceptual Medicine Wheel Orientation Map}

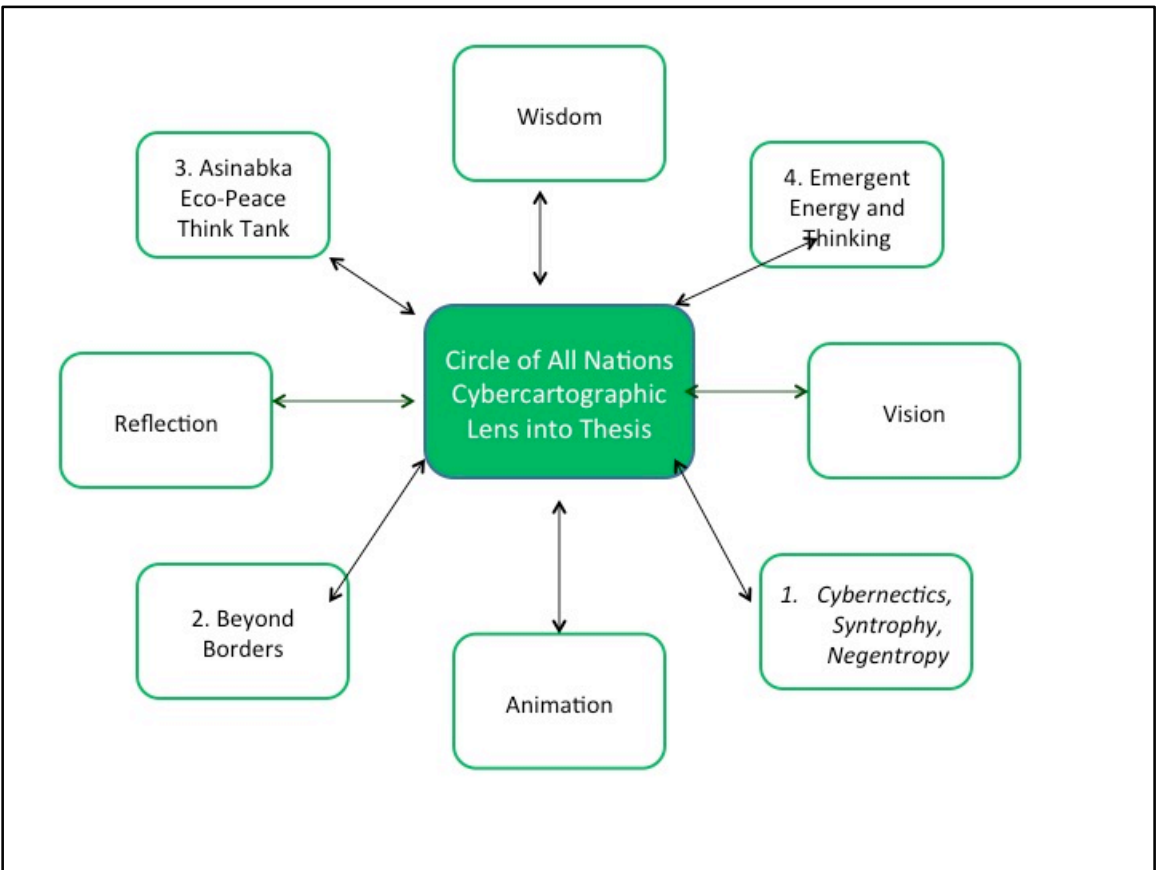

Figure 18. Conceptual Map of Circle of All Nations Cybercartographic Lens into Thesis 


\subsection{Discussion, Analysis and Observations}

In this section of the thesis, the narrative focuses on movement, journeying, and WC's travels during his lifetime, and his continuing presence in the cyber world.

\subsubsection{Cybernetics}

From cosmic cyclical orientation to identification of self as Mamiwinini, to the iconic symbol of birch bark canoe, the inherent theme of movement is the life-breath of WC's discourse. While gyber is word associated with a new phenomenon, the internet, a Wikipedia search reveals that it is derived of ancient Greek roots of I steer, drive, guide, act as a pilot, implying being good at steering, being a good pilot, linked with 1830s French cybernétique, the art of governing. Cybernetics is applicable when a system being analyzed incorporates a closed signaling loop, originally referred to as a "circular causal" relationship - that is, where action by the system generates some change in its environment and that change is reflected in the system in some manner (feedback) that triggers a system change. Cybernetics is relevant to mechanical, physical, biological, cognitive, and social systems. It is most interesting how this idea runs parallel to the syntropic/negentropic theme with which I have been associating WC's Law of Nature/Wampum Belt agency.

This root of the cyber concept allows me to argue that the mapping/journeying/steering motif begins with WC's birth, and key trails emerged years before he became embedded in the modern cyber world. I also examine this looping feedback pattern in the context of animal teachers. Much Indigenous knowledge is obtained through intense observation, interrelationship and reciprocity with the animal world.

WC's birth name, Ojigkwanong, Morning Star, aligns him with the cosmic cycle of movement, and references the primacy of the sky world in the ancestral knowledge generation process. He was never confined to the reserve through his entire life, having 
moved to the bush regularly through his childhood and youth (also thereby escaping confinement in residential schools). From the mid-forties, he travelled across the country to engage in Indigenous revival, and animate gatherings.

WC demonstrated his own agency in his refusal to be defined by borders. On the one level, this was land-based physicality. He was at home on the Kitigan Zibi Reserve and beyond, in Maniwaki, and Ottawa, in Quebec and Ontario, and in Canada and the United States, and he was also a global traveller.

As an adult, he worked in the lumber business as veneer log buyer for many years, travelling across the American North East, working in Ontario, Quebec, New Brunswick, Nova Scotia, Maine, Massachusetts, Vermont, and New York State, while also serving his community as chief appointed by nomination for over nineteen years (he never campaigned for leadership; rather, he just served his community as requested. He was often away during the elections, and his wife would call him and say, "Well they voted you in again!"), and Supreme Chief of the Indian Nations Government. It is rather phenomenal to realize that winter or summer, he left home on each week on Sunday afternoon to head to New Brunswick, then return on Saturday via his Quebec City stopover with Jules Sioui, Secretary of the North American Indian Nations Government. He drove long and fast, and told tales of out-pacing police on his tail. Being Indigenous was an advantage to the lumber companies because he could buy the logs in both Canada and the US. When I reflect on his stories of racism where he reported not obtaining rooms in motels, not being served in restaurants, and not being able to access washrooms, I suggest that his determination to keep going and break borders down was enmeshed in part with the strength generated by movement denied others confined to the reserve. In part this also explains why his story cannot be confined easily to academic definitions of environment, to project his thinking 
adequately.

By the mid sixties, WC was also travelling internationally. Over the ensuing decades he journeyed to France (three times), Denmark, Switzerland, Mexico, Japan, South Africa, and the Bahamas. I travelled with him throughout the last fourteen years of his life, and other than the international travels, we drove across the American North East several times. We also drove between Ottawa and Maniwaki literally thousands of times. In retrospect, as I review our routes, I note the historical significance of the sites: Pipestone, Minnesota (sacred stone site); Turner's Falls, Massachusetts (Indigenous meeting place and colonial war), Shubenacadie Reserve Nova Scotia (residential school), Plaster Rock, New Brunswick (mirror illusion), Lake Superior Copper Mine (ancient travels) amongst countless others. I register this to note the importance of constant movement and travel to his very being and the importance of place to his knowledge and priorities.

WC served as spiritual guide for the Sunbow Five $W$ alk to raise awareness of Indigenous issues, environmental crises and to take healing Indigenous prayer and ceremony to land besmirched by blood and warfare. The Sunbow Five walk commenced at First Encounter Bay on June 23, 1995 and people walked across the continent arriving in Santa Barbara, California on 3 February 1996 (the site of the most notorious of Catholic missions). WC, whom I have called First Walker (in view of the contemporary walks of Indigenous Peoples, for example Cree youth and women's water walks, and the October 2017 Israeli and Palestinian Women's Walk Waging Peace) joined the walkers at seven points of the journey. This journey took place across the United States, but WC from Canada was there, reaffirming his identification with the continent of North America. The wampum belts that originated with the quahog shells of the Cape Cod area from before the coming of the New Peoples to North America, were immersed in the Pacific Ocean, attesting to their temporal 
and spatial agency and actant significance. Writer Stephen McFadden wrote about this experience as the Seven Fires Odyssey in an on-line book www.8thfire.net. There were divisions amongst the walkers by the end of the journey. WC told me that he had had a vision about that tri-partite division at the beginning of the walk (he drew the vision) yet he persevered with project; this is one more illustration of the syntropic governance of his performativity. Later, many of these walkers helped animate the Circle of All Nations gatherings from 1996 till his death in 2011; there were Sunbow Five Walkers from the United States at the 2012 Theresa Spence hunger strike at Victoria Island, on the Asinabka Sacred Chaudiere Site, attesting to the negentropic cycle that is still in the process of manifesting. While on the Sunbow Five Walk, WC did a pipe ceremony at the motel in Memphis where Martin Luther King was shot. Years later he was presented with the Martin Luther King Dreamkeeper's award; the organizers were unaware of WC's earlier connection with that leader. Like his receipt of the Wolf Project and Harmony Awards for promoting racial harmony, this acknowledgement on Parliament Hill enabled him to register that Indigenous welcome to Canada's increasingly diverse population, including to the Black slaves whose arrival in Nova Scotia and actually on WC grandfather's own land, came before Martin Luther King. I reference these stories to draw attention to the land based integration of social justice, land, people, healing and environmental priorities in WC's work - and I suggest that this Indigenous approach to a wholistic understanding the Laws of Nature is what he was driven to present to the academic domain of geography. This too warrants further investigation.

\subsubsection{Agency of Animals}

I now examine relationship with the animal world, to expand on the consideration of multiple pathways to knowledge. For WC, the profound teachers emerged from the world 
of nature, and representatives of the animal world played critical roles in the epistemology: relationship was the threshold to knowledge acquisition. Of course, beyond this, there is the intimate knowledge of animal anatomy. By comparison, note the history in England, from 1066 and the time of William the Conqueror. Common people were prohibited from hunting and killing animals. This became the prerogative of nobility; in France, the "de" in names identified those who were authorized to kill. Indigenous hunters were intimately acquainted with anatomy and physicality and they knew how to work with bones and sinews. WC's wife's grandmother, Kitchi Mani Jacko Kishkanakwat, was a noted midwife and healer in the Buckingham, Quebec area, healing non-indigenous peoples as well. In 2015, Caroline Andison, an indefatigable local Ottawa valley researcher and non-academic historian, and I had a meeting with a friend of hers from Buckingham. David Bigelow Thompson, who was in his late sixties, recalled the skill and power of Kitchi Mani Jacko, his grandparents having talked about her mending of bones, stitching of wounds, and use of hot coals to cauterize wounds. WC himself had smallpox during his childhood, and it was the medicine man Pierre Dube, husband of his paternal great aunt, who healed him. The town doctor from Maniwaki, Dr. Mulligan, sent hopeless cases to him for medical services. There were many statements of how animals guided Indigenous healers to medicines. WC's own recovery from nearterminal cancer involved a turn from western surgery to herbal medicine made by his wife, Mary Smith Commanda and their friend, Margarite Budge.

Agentive animal subjectivities are examined in The Animal Gaze (Woodward 2001). Here, Wendy Woodward, a literature professor, examines the construction of nature via animals in literary works. She scrutinizes the way an animal looks at a human and how a human responds to such a gaze in South African fictional and non-fictional writings, wherein the animal actively claims subjectivity in the encounter with the human in a moment of 
interspecies communication, from a bistoricized culture embedded in Judeo-Christian and Cartesian separation of animal from human, with animal deemed unfeeling and therefore experimented upon, to a culture now enlivened with debates about animal rights and ethics, and dark explorations of unspeakable things via story that appear to seek resolution via interface with the Indigenous shaman. She references:

(a) Derrida's 2002 essay, “The Animal that therefore I am” (in Woodward 2001) which contradicts Cartesian philosophies of animals as creatures lacking sentience or feeling, and posits human ontologies or theories of being in response to the gaze of an animal, with animal as agentive, bringing the human to new awareness of self;

(b) Carol Adams' consideration (in theories of knowledge) of knowledge production as "relational epistemologies" in narratives of kinship between humans and animals (Adam 1995 155);

(c) Patrick Murphy's observation that in the traditional beliefs of many Indigenous cultures, agency is extended to both human and animal in an acceptance of interconnection between beings that is heterarchical or egalitarian, rather than hierarchical (Murphy 2000 87-88);

(d) Deleuze and Guattari's (2004) concept of 'becoming animal', intrinsic to the shamanistic ontological construction of "soul" or energetic connection between species: which they suggest is not a metaphoric process but rather one in which identities become "deterritorialised" through immanence rather than transcendence, consistent with Urpeth's articulation of "animal-becoming" as a radically nonanthropomorphic and non-theological concept of nature, with movement in continuum beyond threshold space in the interconnection; and further; and

(e) Woodward also draws on ecological philosopher Val Plumwood's analysis of a "master consciousness" which sees humans outside of nature, thereby conceiving of nature as alien, re-presenting nature, with the other only recognized to the extent it is assimilated to the self, because the "master consciousness" cannot tolerate the "unassimilated others" (Plumwood 199353 in Woodward 2011)

Moving beyond western anthropocentric thinking, she explores Derridian contemplations of animal power and capability. This is followed by a two-fold exploration of relational epistemologies as featured in Indigenous shamanist tradition, and re-presentation of animals as sentient beings deserving of justice (Woodward 20011 18). In fact, in the flow of her analysis, it becomes apparent that the horrors of the colonizers' relationship with animal, wild and domestic, seeks healing and resolution via accessing the Indigenous.

These interrogations are pertinent to a further auto-ethnographic examination of WC's 
discourse. While justice imperatives informed WC's historical narrative, an understanding of the geo-narrative ideological underpinnings of his relational ontology and epistemology draws from a shamanistic inter-species energetic connection, primarily reflected in an animal epistemology derivative of deep observation, extended study and relationality. This is sometimes rooted in the animal gaze, sometimes energized, in Indigenous language, as shape-shifting, and sometimes manifested as a complex of the agentive and animative.

This agentive/animative manifestation is critical in the Commanda rootedness in ancestral relational (versus dominion) ideologies. Repeated iterations of particular energy shift, epiphanic, or luminous moments in his life reflect his foregrounding of key moments of interaction with iconic representations of the animal world. Note, with his repeated reference to Mother Earth, WC is already presenting himself as a member of her animal (as compared to stone or plant) world. Spider, Wolf, Snake and Bird figure in these multiply narrated moments. Many representatives of the animal world were critical to his way of knowing, grounded in animal epistemology, amongst them Eagle, Condor and Hummingbird, Turtle, Squirrel, Fisher, Beaver, Muskrat, Buffalo, Bear, Skunk, Fox, Moose, Frog, Snake, Eel, Spider and Moose. For example, there is reference to Giant Snake and its importance to life (as compared to the evil of the biblical snake) in the earliest recordings of Algonquins stories. Snakes were not feared but respected. They were known to occupy the deep waters and cross land to pass from one lake to another and to serve a purpose. In contemporary times, a monk reported to WC about seeing one plunge in and out of the water in the James Bay; the Cree people affirmed they had seen that before. One crossed the highway to WC's brotherin-law's lake in Quebec, stopped trucks, and was reported in the media in contemporary times. WC himself recalls a canoe trip with his friend Ray Tenesco, at one place, they had to portage their canoe, and walk over a giant $\log$; when they returned, WC realized the $\log$ was 
no longer there. Science is now discovering the life generative function of the whale's trophic plunge into the photic zone of the ocean, the deposit of fecal plumes and iron to stimulate generation of plant plankton, at the same time sinking carbon. Just because western science is now proving things with the advance of technology and global knowledge sharing does not mean Indigenous peoples had not already understood the significance of such findings through their observational and knowledge generation processes centuries ago. Increasingly, modern science affirms this Indigenous knowledge. But Indigenous knowledge also ventures into zones of incommensurability. I referenced WC's discussion in Ojigkwanong of his spiritual awakening following his relational exchange with the Singing Spirit Bird, the Rosignol, when dying of cancer; the other storied creatures in WC's intelligence of nature narrative are Spider and Wolf. I shall now reflect on them briefly.

One evening, when we were watching the Spiderman movie, we began to wonder how Grandmother Spider got taken over by Spider Man! WC related the following story:

"Spider is more than an insect. One very cold winter day, I went to my workshop; there was a big black spider on a piece of wood. You never see a spider in a cold shop in the winter; I had not lit my woodstove yet. I knew it was more than a spider. I went off for my walk. I used to always walk before breakfast. When I returned, Spider had disappeared. I decided to use the wood it was sitting on to make my drum. Years later I went to a gathering in Manitoba. Before the gathering, some elder said, there will be a man coming from the east; he will carry his pipe in an otter skin bag; he will have a crooked drum. That was me! Somehow, someone who did not know about me knew I would be coming: me with my crooked drum and my otter skin pipe bag!"

WC was very matter-of-fact with such recountings. I learned to put my dominant left brain on hold, and just listen. WC's knowledge of Earth emerged with ancestral attunement to the Laws of Nature. The extensiveness and depth of this knowledge is examined in the context of the geological development of his homeland. But there was also an inner understanding of relationship with the metaphysical, and furthermore, understanding linked with the science of nature, the intelligence of nature, and its relationality. I note in William's 
discussion of his encounter with Spider, that teacher, instructor, animator, tree, insect, eight legged, season, distance, movement, time, pacing, and creativity constitute elements of a nuanced understanding of life. Even the quirkiness of a crooked drum, the trickster's nudge away from perfect consolidation, is introduced in the earth-based agency of drum. This Spider drum underpins the nebulous and intangible quality of his Circle of All Nations work. Metaphysical and physical embrace, and both generate and animate knowledge and meaning in an integrated fashion. Indigenous peoples used the very strong spider webs in medicines for wounds. We have discussed elsewhere the relevance of drum as representative of the heart beat of Mother Earth. It is also intriguing to note western science's recent discovery about spider webs, in the context of WC's drum. Each string in a web is a different length and presents a different vibration, transmitting specific knowledge to the insect. The following paragraph is another journal reflection excerpt about sound, beat and relationality: I include it to illustrate the stream of consciousness knowledge generative academy I entered with WC, where I learned to integrate an understanding of geo-political humanities in the creation of the multi-faceted Circle of All Nations epistemology.

William made his first memorable trip down to the city by train - he was about fourteen, and he bought himself a pair of shoes and a violin from a pawn shop - he was already well accustomed to earning his keep in the new century. The fiddle and the square dances replaced the indigenous dance and drum now prohibited by government edict, and he fiddled till his thumb was amputated after an accident (Indian Affairs doctors got paid more for amputations, he told me - that's why you see so many native people without fingers; consider the implications for and individual's spirit/art relationship and development in the context of environmental knowledge); music, the beat of life, was ingrained in him; monks taught him to read notes on a four line staff and to sing acapella; he sang Ava Maria in Latin to priests and bishops at a conference on nuclear arms and spirituality; and in Germany, his host family were startled to find the old native man recognize Andre Bocelli's voice; he tapped his foot to the beat of Bruce Springsteen, especially liking The River, of George Michael, he said, he's in command of his own agenda; like Johnny Cash, he liked to dress in black and like Squirrel, said I won't back down; he enlivened his Circle of All Nations gathering by bringing in the Lac Simon Drummers, and his tears fell to some of Brad Paisley's songs (Thumbadoo/Commanda Unpublished Joint Journal). WC also orchestrated his own farewell concert; unexpectedly, fifty youth from a boy's choir from Austria, and 
separately, Marc Vella, La Caravan Amoureuse, a musician from France, arrived with his baby grand piano and fifty people from France, and WC's funeral ended in a fusion concert.

I discuss the shamanistic relevance of inter-species interface further. It includes of important discussion of Zulu shaman and writer Credo Mutwa, whose acquaintance WC made in 2001, in an intriguing hodological journey.

The threads and trails that emerge in multiples streams of thought like this Molly Bloom (in James Joyces's Ulysses 1922) narrative at any point in the WC discourse lend themselves to further critical analysis in the future - they span time, space and incredibly diverse knowledge exchange. Here, thought dances beyond borders and boundaries in the spirit of Ginawaydaguc relationality and co-creation of knowledge is sparked by differences and syntropy.

This suggests something of the spiritual gateway to knowledge. It is not sacred. That is a more a qualitative description of something like a place, an emotion or an item. For WC, spiritual is the funnel into a particular way of knowing and of knowledge acquisition. Recall that Circle of All Nations has articulated four trajectories to knowing: the emotional, the physical, the spiritual and the mental. Ceremony, creativity and the senses serve, as it were, as fluids of different viscosity, to permit deeper penetration via each of the trajectories. Integrating reflections from the multiple trajectories generates more knowledge; deep exposure to nature, and passage via multiple pathways increases the knowledge. This is the type of Indigenous knowledge geographer Wade Davis refers to in The Wayfinders: Why Ancient Wisdom Matters in the Modern World (CBC Massey Lectures 2009). There are directional arrows pointing to this knowledge trail. But there are dangers. And there are no short cuts. A journey must be made.

Woodward notes that African beliefs and practices are vividly informed by kinship 
between humans and nonhuman animals. In many creation myths, both emerged from caves or the underworld; spirituality is of and below the earth; it exists in the everyday because it is embodied rather than transcendent and is part of nature. Animals are also links with ancestral spirits, and speak their language. Animals and ancestors stay anchored to earth. Honouring and ceremonial practices contribute to the making sacred of the earth.

Zulu shaman and writer Credo Mutwa argues: 'When an animal is killed in Africa, that animal takes a large slice of African knowledge into oblivion with it because most of the knowledge that Africans possess is intimately intertwined with the animal life as well as the plant life of this continent' (Tucker 2003 9). This is not different from WC's advocacy of Mother Earth and his passionate challenge of environmental abuse. He, like Mutwa, advances the intrinsic value of Indigenous lives and knowledge, and the world's desperate need of the same, and hence his political campaign of making relations.

WC had a relationship with Wolf, this forged in the sense of the subjectivity of Wolf. I am presenting an excerpt from an unpublished Joint Journal, started well before 2006, (perhaps 2003), this apparent because on the top of the page, I had hand written, in an obvious moment of reflection: February 25, 2006 "So funny, starting to rework this today and WC said, what is it going to be (to guide us today) Wolf or Beaver - it was Wolf?'

Well I note that we had also identified the Preamble of our Joint Journal with Wolf. The section is entitled Wolf Finds $M e$.

I was about fourteen. I was out in the bush with my father. He sent me out to check on our trap line across the lake. It was a cold clear winter's afternoon as I returned to our camp with my dog, Rover. I was making good speed on my snowshoes. All of a sudden, my little dog started growling low - and bopped on to my snowshoes. I shook him off. He jumped on again. He continued growling. I picked bim up and held him against my chest. I turned around and saw four big dogs (timber wolves following us).

They were so beautiful - heavy, silver fur shining in the paling sunlight. They circled us and then lined up, two on each side. I stood still, silent. He pauses a moment, musing; his still overwhelmed by the immensity of their presence. They stared at me intently, thrusting their noses into the air as they sniffed deeply. Then, after the longest time, the biggest one, he was as tall as a deer, he rolled 
onto his back in the snow and wriggled around, his legs cycling into the darkening sky. Then he bowled long and loud, shook bimself up and down and took off into the distance. His companions followed him. They turned and looked at us, then disappeared into the night.

We continued on our way. When we reached the camp and I told my father about the big dogs, he asked if I had been frightened. I had not. He had been so awed by their presence, their grandeur, that it had not occurred to him to be afraid. In fact, he must have been so taken by them that he quietened his little dog's fear, and they all, wild, tamed and human, were caught together in one bubble of time. My father stared at me. They smell fear, you know, he said. Did he see the potential for his oldest son to really learn the ways of the past?

They came to visit again. Again, my dad and I were out alone, and we had set up our camp with our back to the lake. The dog started to growl and we knew something was outside. My dad crept out of the tent and had to walk to the back. The four wolves were at the lakeshore. We had no gun. He was sorry about missing the skin he might have taken. I was glad we did not shoot them.

I am now over ninety. They are still alive.

It is so obvious that this is a powerful memory for the elder. That experience of a wild and remote beauty and presence has stayed with him all bis life. It has informed and guided his life in a fashion that is intriguing to plot.

He is over ninety. He spent the first part of his life bunting an trapping in the bush, most with his father, sometimes with his two younger brothers, Alex and Lowizon as well, and sometimes with his much older brother in law, Joe Jacko.

His legs are still hard with muscle, and he still walks straight, with an indomitable determination, despite the fallen arches, the damaged toes, the failing eyesight. People always note his purposeful stride.

The energy of the Mamiwinini, the nomad, pounds through his veins, attuning his footsteps to the memories of the land and the spirit of its creatures. The connection had been so seamless, even in his youth, that he could calm the fear of his dog and fall in absolute synchronicity with the intent of its wild counterparts - the intent to cement a bond already forged in ancient memory lines. Is this the marking of a shaman, I wonder - the encounter with the wild, the unknown, with a mask of death? He was fearless, but more than that - he knew no fear. And so be was at one with the wind in the mystery of the moment.

A few years ago, some medicine healers did a ceremony over him; they sensed the spirit of Mohigan, Wolf, within him and they wanted to cleanse him. I was concerned. I had long felt that his wolves were protecting and guiding him and I did not want them disturbed where they had interwoven their essence with his life line. ... So I focused my being on the power of his story from his youth. To me, they were palpable in the energy in the room. Did he know, did he remember them, I wondered. He came out of the sleep, the trance, and them he began to tell the healers gently about his encounter with his wolves. Perhaps they learned something even deeper about their heritage. (Thumbadoo/Commanda First Unpublished Joint Journal 2007)

(As an aside, I note wondering whether I would hear his wolves when WC died. Well, 
his funeral on Friday August 5 led directly into the 2011 Circle of All Nations Gathering, and in the evening we screened the documentary, Canada The Movie, by John Thompson, the friend of Kirk Wipper, Founder of the Canadian Canoe Museum. He had interviewed William Commanda for some portions. Well, it opened with a scene of a northern winter landscape - and wolves! I smiled thinking WC's wolves were howling him home!)

\subsubsection{Asinabka Eco-Peace Think Tank}

Over the course of this research study, which commenced in 2012, the word sacred has taken on particularly profound significance, demanding an unanticipated degree of praxis, with respect to a twenty-year Circle of All Nations project of WC: his legacy vision for the Asinabka Sacred Chandiere Site (www.asinabka.com). While this is not the key focus of the thesis, nor of this portion of the thesis, I note that the site referenced pertains to the Chaudiere Falls and several islands in Canada's capital city, in the heart of the National Capital Region. I offer the first historical description of the place, excerpted from the 2010 project proposal presented to (and subsequently endorsed by) the City of Ottawa, by WC, with text by Samuel de Champlain, the first European explorer to the area, formatted in italics here. It shows the interplay of history, the intangible, sacred, ceremonial and environmental in WC's thinking.

From time immemorial, Asinabka, the Place of Glare Rocks at the sacred Chaudière Rapids, has served as a special spiritual meeting place, a special place of council fire, for the Indigenous Peoples of North America. For countless seasons, the Mamiwinini, my ancestors, gathered at special meeting places across the continent meeting places that were on waterways that served as their highways, and islands that served as their half-way houses, marked by thundering rapids. The four elements marked these as places of power, places of passion. The Chaudière Rapids were especially significant - they reflected the shape of a pipe, in this place of glare rock, and the rising vapours were an expression of the prayers of the people rising to the Great Mystery on the wings of the wind. The Mamiwinini consecrated the place with prayer and this was noted in one of the earliest paintings of the Asinabka area: it is painting of three native men in different outfits, perched on an outcrop of rocks, smoking the sacred pipe, and offering tobacco to the whirling rapids below them.

This area was an important destination for the Algonquin Peoples, because it was the site of "their chief Hunting Grounds, Sugaries and Fisheries", and this was noted in 1824 in the records of the evolving country (Appendix R to the 23 volume of the Journal of the House of Assembly of the Province of Lower Canada). 
The painting of 1613 is complemented from the earliest annals of documented history of the area in the journey of Samuel de Champlain on la riviere des Algoummequins. Champlain, geographer, explorer and colonizer, founder of Quebec and indeed a forefather of Canada, was the first person to publish maps of the Mighty River and its topographical features (though some researchers suggest that the original descriptive information was authored by Etienne Brule, the first explorer in the area (Robertson Walking into Wilderness 2010):

"The water falls ... with such impetuosity on a rock that with the passage of time it has hollowed out a wide deep basin" wrote Samuel de Champlain in describing the Chaudière Falls which he saw for the first time when journeying up the Ottawa River in 1613, "...The water whirls about to such an extent, and in the middle boils so vigorously, that the Indians call it Asticou* a kettle. This waterfall makes such a noise in this basin that one can hear it from more that two leagues away." (Champlain in Kennedy 1970 7) (*Note - The Algonquin word is actually Akikpautik for the pail rapids; further, it was WC's opinion that the Astic was a misspelling of Akik, derived from other dialects)....

"After having carried their canoes to the foot of the falls, they assembled at one place, where one of them with a wooden plate takes up a collection, and each one of them places in this plate a piece of tobacco... the plate is placed in the middle of the group, and all dance about it, singing in their fashion; then one of the chiefs makes a speech, pointing out that for a long time they have been accustomed to making this offering, and that by this means they are protected from their enemies... the speaker takes the plate and throws the tobacco into the middle of la Chaudiere (kettle), and they make a great cry all together" (Champlain in Kennedy 1970 9).

Champlain spoke disparagingly of the natives' superstitions, praying at the tempestuous river, but soon enough, he himself was in difficulties for his canoe had spun broadside in a whirlpool and

"had I not luckily fallen between two rocks, the canoe would have dragged me in since I could not quickly enough loosen the rope that was twisted around my hand, which burt me very much, and nearly cut it off. I cried out to God and began to pull my canoe toward me...As for our Frenchmen, they did not fare any better, and several times were nearly lost. ... Having escaped, I gave praise to God, beseeching Him to preserve us" (Champlain in Legget Ottawa Waterway Gateway to a Continent 1975 72).

Thomas C. Keefer, a civil engineer noted in 1854, "There is scarcely a portage or cleared point, jutting out into the river where you do not meet with wooden crosses, on which are rudely cared the initials of some unfortunate victim of the restless waters...". "In a prosperous year, a thousand men are afloat on loose timber, or in frail canoes, and as many as eighty lives have been lost in a single spring" Keefer in Kennedy 1970 9) 
The vignette of Champlain's first famous foreigner's encounter with the Mighty River reveals the attitude with which the sacred watch of my ancestors was dismissed as trivial and superstitious; we and our land have experienced great pain with the imposition of other Gods upon us. In 1800, my ancestor, Chief Machecewa met the first settler in what was to become the capital city. I quote my comments of February 23, 2009 regarding the Navigable Waters act at this point, since they are relevant to our unceasing concerns:

I am a ninety five year old Algonquin from the Ottawa River Watershed, and my passion and commitment over many decades has been to draw attention to the ceaseless plundering and destruction of the grand natural resources of my ancestral lands, the deepening environmental crisis across the globe, and its impact on our lives and health; to promote environmental stewardship; and to warn of the crucial need for Indigenous wisdom in reclaiming a balanced relationship with the world we all live in. (Commanda 2010 Vision for Asinabka 2010 Feb 22 in www.asinabka.com).

WC developed a vision for a fully inclusive Indigenous healing and peace-building centre at the Asinabka Sacred Chaudiere Falls site, on un-surrendered, unceded Algonquin territory. It lies within the National Capital Region, between the Ontario and Quebec borders, in Canada's capital city. It was developed by thousands of Indigenous and nonIndigenous peoples over the course of twenty years, including world renowned Indigenous Architect Douglas J. Cardinal, who spent decades developing conceptual designs for the site, and it was endorsed by all formal Algonquin communities, the federal government and the City of Ottawa. Unfortunately, shortly after his death in 2011, Windmill Developers and Domtar developed plans for condominium development, and the City of Ottawa rezoned public lands to permit privatization at this sacred, geologically, culturally and historically unique site. This has been challenged heatedly in the courts and elsewhere over the past four years by multiple parties, both aligned with and independent from the Circle of All Nations, all variously inspired by WC over multiple decades. The Student's Union at Carleton University has also been engaged in this effort. Many view the development plans as a neo-liberal capitalist seizure of power in a vicious repetition of the colonial land grab pattern, where voices are conflicted, compromised, coopted, corrupted and silenced, and where 
privatization and corporate interests and land appropriation dominate once more at the singular sacred site. Foucault's discourse analysis drew attention to power, knowledge, truth and truth effects; here, WC's legacy vision for his ancestral lands is challenged by an intermeshing of money, power and politics. With respect to social media, preliminary evidence indicates that the attempted repression of the Commanda Asinabka vision has in fact contributed to his continued vibrant presence in the cyber domain. There is increasing exposure to the foundational issues of colonization and its ramifications, and to the continuing practices of colonialism that ignited WC's own Indigenous movement of resistance, reconciliation, and reformation. The controversy has planted WC's work and vision firmly in the academy, and numerous masters and doctoral students, including international students, are actively researching the file. While it is not the focus of this thesis, note that the www.asinabka.com site and Facebook page William Commanda Legacy Asinabka hold all the critical Circle of All Nations documents on this legal challenge, and historical documents on the development of the vision. Elements of this project also constitute part of the digital atlas records.

Unfortunately, the struggle is also viciously divisive at multiple levels, and losses and damages to multiple parties, including the global stage, is incalculable to date. The fight against development is now being led by young and old people, Indigenous and nonIndigenous, church groups and social justice activists; many of these people never knew William Commanda. Social media, much like the Gutenberg Press of Martin Luther's day, has awakened the country at large to a tremendous amount of its shameful colonial history. So has national Indigenous presence on such critical matters as youth suicide, Murdered and Missing Women, Truth and Reconciliation, Idle No More activism. It is ironic but now, the thinking establishment and church communities would like to see the manifestation of the 
Commanda Asinabka Sacred Chaudiere Site Legacy Vision, since it offers a respite from colonial shame; their hostility towards the developers appears to be on the rise.

The issue also illustrates the intransigence of market capitalism, where justice is deputized to serve corporate systems. Over the past four years, it has not been possible to have a single open public discussion of the Commanda vision with authorities (City of Ottawa, National Capital Commission, federal ministers, and other political leadership, including Indigenous). Government responsibility for consultation has been assumed by corporations, and legal challenges are dealt with by prescriptions of declining jurisdiction. By contrast, as with cultures that have no word for justice, and are instead instructed by other cultural structures, Aupilaarjuk discusses the concept of Maligait, an Inuit customary law word, that works much like wampum law: "When I think of paper I think you can tear it up and the laws are gone. The maligait of the Inuit are not on paper. They are inside the people's head and they will not disappear and be torn to pieces. Even if a person dies, the maligait will not disappear" (Aupilaarjuk in Simpson 2008 131). This is the type of integrity that WC wanted to see ignited at the Asinabka Sacred Chaudiere Site. Contemporary Indigenous academics and writers like Lynn Gehl (Mkadengwe: Sharing Canada's Colonial Process through Black Face Methodology 2014, The Truth that Wampum Tells 2014, and Claiming Anishinaabe:

Decolonizing the Human Spirit, 2017), Bonita Lawrence (Fractured Homeland: Federal Recognition and Algonquin Identity in Ontario. 2012), and Noreen Kruzich (The Ancestors are Arranging things 2011) are drawing new attention to present day colonial land appropriation practices, including at this site.

\subsubsection{Contemporary Work Generated by William Commanda's Legacy}

Circle of All Nations continues to animate WC's work through relationship building events and academic presentations and interface; several events focus on environmental 
stewardship and peace building each year.

During the past two years, WC's work, knowledge and pedagogy have been presented in workshops of the Association of Canadian Geographers at Carleton University in Ottawa, at the American Association of Geographers Conference in Boston, and at two conferences at the University of Ottawa, and at two Child and Youth Care Conferences, one in South Africa and one in Thunder Bay, Ontario.

WC was also a central website presence in the International Anthropology Conference hosted by Canadian Anthropology Society and International Union of Anthropological and Ethnological Sciences at the University of Ottawa in May 2017 (http://nomadit.co.uk/cascaiuaes2017/en/index). Representatives from both University of Ottawa and Carleton University participated in the organization of the conference, convened under the leadership of Professor Scott Simon, who had visited WC and participated in a few Circle of All Nations gatherings, and who then recruited me to help with the conference planning. Thus Circle of All Nations played an active role in the conference, including inputting into the development of the conference theme, Movement. Note that WC's connection with the University of Ottawa was celebrated in the conference website, which reached thousands of international academics and anthropologists, thus centering and igniting a new relationship with Indigenous. In addition to the inclusion of Indigenous welcome by WC's daughter, Evelyn Commanda Dewache, drumming and Indigenous commentary, and community outreach, Circle of All Nations managed an information booth, and organized a double session workshop comprised of academic and non-academic presentations. We also organized an excursion to the Kitigan Zibi Anishinabeg First Nations community, and a teaching session at William Commanda's own teaching lodge. For the fifty international participants, this was the highlight of the conference. Many of them 
are now a part of his Facebook community. Dr. Georges Sioui, who launched the Aboriginal Studies Program, noted the pivotal role William Commanda and Circle of All Nations played in the development of the program.

The following quotation is from the conference website, which reached eight hundred participants globally. It also endorsed the theme of borderlessness.

\section{In celebration}

We remember and celebrate the life of Algonquin elder and spiritual leader, Dr. William Commanda, OC (1913-2011), honoured at University of Ottawa with the presentation of his first Honorary Doctorate Degree, and the creation of the William Commanda Hall. He commenced his Circle of All Nations work and gatherings formally in 1967, to promote protection of Mother Earth, respect for Indigenous wisdom, racial harmony and peace building, locally, nationally and internationally. Fifty years later, his legacy continues to inspire. Describing himself as Mamiwinini, a nomad, he said "I am in the earth, wind and waters; I am as the bird flies and the wind blows and the water flows - Ginawaydaganuc - We Are All Connected" - a fitting epithet for a conference on $\mathrm{Mo}(\mathrm{u})$ vement.

\section{CASCA/IUAES2017 Conference in Ottawa}

International Union of Anthropological and Ethnological Sciences (IUAES) and Canadian Anthropology Society (CASCA)

The Conference/InterCongress was hosted at the University of Ottawa, on the unceded, traditional territory of the Algonquins of the Ottawa River Watershed. Canada's capital city is built on the banks of the Kitchisippi, the Great River, the Ottawa River; the Sacred Chaudiere Falls and Islands have served as a meeting place of great spiritual importance to the people of North America since time immemorial.

770 delegates attended the conference over the five days, which consisted of 126 panels, more than 700 papers, nine roundtables, two keynotes, three plenaries, several workshops and meetings. (CASCA/IUAES2017 Conference website)

The integration of Indigenous, community at large and academia continues,

emergent from the conference moment. Of interest here are the academic links. A former

University of Ottawa student, who had used the Learning from a Kindergarten Dropout

(Thumbadoo 2005) during her university years, is now teaching criminology in a university in

British Columbia, and she wanted copies of the text for her academic work. The book is 
profiled in the University of Ottawa Library 2017 commemorative poster. A Carleton legal studies student is now researching Circle of All Nations colleague, the late Indigenous justice and rights advocate, Donald Marshall Junior; and he and L. Jane McMillan, PhD, Chair and Associate Professor Department of Anthropology, St. Francis Xavier University, (who is now publishing a book about Donald Marshall through the University of British Columbia Indigenous Publishing stream), made a presentation about his historical contributions to Canada (the Donald Marshall Justice Inquiry, Aboriginal Treaty Rights/Fishing, and advocacy to place the American Eel on the Species at Risk list) at the Circle of All Nations workshop.

Finally I note the opening of the History Hall at the Canadian Museum of History for July 1, Canada's $150^{\text {th }}$ birthday. As noted elsewhere, WC has had a long association with assertion of Indigenous at this singular architectural masterpiece created by world renowned Indigenous architect, Douglas J. Cardinal. A special panel profiling WC is now a permanent part of the exhibit. Further, his words We must come together with one beart, one mind, one love, and one determination, to create a Circle of All Nations, a Culture of Peace, greet all visitors from the pillar at the entry.

\subsection{Concluding Remarks}

WC was genuinely borderless in his inter-relationship with the entire diversity of peoples beyond Algonquin. In a birthday book co-authored by his friends when he turned 90, Valerie Pouyanne, who produced his Good Enough for Two canoe DVD wrote, "At one moment I realized that William's face was reminding me of my father. As I was sharing it with my neighbor he answered me that William was reminding him of his mother, a few minutes later a woman took the microphone and told that when she was looking at William she was seeing her grandmother. Suddenly it was all a family gathered in William's face. 
Maybe it is the face of universal and unconditional love that everyone recognizes in William and that gives him this friendly and close air" (Pouyanne in Circle of All Nations Publication 2003 28). On September 16, 2017, three people saw woman in William Commanda's photograph. This is the second time I have noted gender borders being erased. Perhaps this is consistent with what is becoming more public in the gender recognition today in the global world, or perhaps it is reflective of the quest for the divine feminine, which, in WC, was Mother Earth. For over twenty years, WC has shown people maps of land form changes predicted by certain scientists. This spring, the Laws of Nature are intervening to erode borders in the American North East.

The evidence of WC's presence in range of fora, including the cyber, suggests that his discourse remains relevant and compelling and continues to steer in a cybernetic manner.

\subsection{Summary of Findings}

With the advances of technology, researchers are now examining posthuman agency in the digital age. In her article on Posthuman Agency in the Digitally Mediated City, geographer Gillian Rose notes that researchers have drawn on posthumanist philosophies to theorize the agency of the technological nonhuman, while under-theorizing the agency of the human, and she theorizes a specifically posthuman agency both mediated through technics and diverse, co-constituted with technologies, with such sociotechnologies being differentiated, in terms of spatialities and temporalities (Annals of the American Association of Geographers, 107 (4) 2017 779). Rose notes further that much current work examining the digitally mediated city is dominated by an approach that emphasises "the unfolding or evocative power of technology to make things happen" (Kitchen and Dodge 2011 42). Without doubt, the emphasis on the agency of digital technologies in shaping urban spaces and experiences has important things to say about the 'automated management' of urban space (Kitchen and 
Dodge 2011 x). Its lack of attention to other forms of agency, however, especially to what this article has called posthuman, is striking, given that "the ever-increasing amount of technological mediators there surround us have radically expanded our experience of what it means to be human" (van Doorn 2011 536). Thus she concludes that geographers must reconfigure their understanding of digitally mediated cities, and acknowledge the inventiveness and diversity of urban post human agency.

This widening of the lens lends itself to an academic consideration of the presence of WC and Circle of All Nations on the web, Facebook and in cyberspaces occupied by unknown parties. WC's work has been entirely unfunded, and has involved a range of different activities over the years; much of the animation of his ideas has been undertaken by people who for whom these views resonate; but they do not represent formal teams. They reflect different interests and talents and are generally engaged with one or another of his passions, but by no means all. Thus, the thousands of people from across the world who attended his gatherings at his home believe that that is the key thing he did, while environmentalists fighting to put the American Eel on the endangered species list value his leadership there, but may have never been to his home. In the absence of a funding and staffing infrastructure, the capacity to develop a sophisticated website in keeping with current innovations has been limited. Nevertheless, the Circle of All Nations blogs of the 2000s served to evolve an internet community that cohered around the integrity of Commanda global ecocommunity discourse, so it holds an integrity that still resonates. Since his death, the termination of his barrier-breaking gatherings has been a sore loss, and while conferences and workshops are orchestrated to continue the work, they by no means approximate that energetic momentum. The immediacy and global reach of a social media platform like Facebook serves to increase his influence in a new way. Now as individuals engage with his 
ideas, they evolve and strengthen the clout of his original seed ideas and ensure the dispersion, and the attraction of new players to the source. The discussion of contemporary news, environmental and social dynamics is supported by the flow of Facebook platform, and the global connectivity augments the Circle of All Nations priority to support diversity and environmental stewardship.

Screenshots of several Facebook posts are examined in the digital atlas and NVIVO, to identify the consistency in style that ensures a tangible respectful space, no doubt controlled but also safeguarded by Facebook's algorithmic structure. Certainly, his posts generate constant interest; the daily interest from new players attest the continued alertness to the Commanda name. However, other than brushstroke depiction of the WC cyber playing field, it is not possible now to undertake a critical examination of issues that draw the greatest interest in the Facebook cyberspace, to assess the posthuman agency that Rose draws attention to; and in the case of WC's reach, it is not merely the urban but rather the microcosmic global reach that warrants further intensive study.

Throughout his life, there was a curious co-constitution of a groundedness in something so ancient as to appear other worldly, and so ultra-modern. On the one hand, he was a holy man, shaman, carrier of ancient spiritual relics, and mystical ceremony (I often called him the DC elder, directly connected with some unknowable force). At the same time, WC was also enmeshed with exploration and acquisition of every modern technology of the past century (with some things, like the electrical metal steel water heater trough for shaping wood for canoe making created especially for him). From his Underwood typewriter, the slide projector, to camera, video maker, television to the Buick Roadmaster, overhead projector, first IPhone, drills, saws, tiller, four-wheeler, WC had the first in every new product and model, and he learned to use them all. He had an insatiable curiosity about 
everything, and epitomized the penultimate lifelong learner.

Something of this essence is discussed by Pearson, according to whom life is neither a metaphysical notion, nor a semiotic system of meaning; it expresses itself in a multiplicity of acts, encounters and events (Pearson Germinal life: The difference and repetition of Deleuze 1999). Life, simply by being life, expresses itself by actualizing flows of energies, through codes of vital information across complex somatic, cultural and technologically networked systems.

Critical posthumanism researchers, noting global engagement with advanced technology, examine the species-wide blurring of boundaries at the edge of the human, in a digital age in which human identity is being transformed through networked technological intervention, with a large part of our consciousness transferred to "smart" external devises, somewhat like the Sheldrian morphic extension, but now distributed and streamed via cyber technological manipulations. In Critical Posthumanism and Planetary Futures, (Ed. Banerju, D and Paranjape, M. R, 2016), researchers engaged in posthumanism theory now grapple with the existential co-constitution of the human with the technical on the one hand and with the vital and the spiritual on the other, as well as the contemporary historicity of the emergence into practical, social and political consciousness of this condition.

Rosi Braidotti, as Deleuzian feminist thinker, in her discussion of nomad, foregrounds this: nomadic thought provides a new ontology, a re-grounding of subjects in the radical immanence of their embodied and embedded locations. Citing Guattari, she adds that living matter is a process ontology that interacts in complex ways with social, psychic and natural environments, producing multiple ecologies of belonging (Guattari 2000).

In this time of global existential dilemma, then, the posthuman is just the question; the answer is what 'we' are can become. She argues that this answer can only be a practical and pragmatic one: "It is the praxis that aims at becoming a multitude of missing people, 
multiple 'we' becoming-world together amidst the painful contradictions of the Anthropocene moment, when waves of the world history may be about to erase from the sandy shores of this planet the face of a species that will have been our own" (Braidotti in Banerju and Paranjape 2016 29). The patterns of our becoming begin with the realization of the loss of a familiar notion of the 'human', which coincides with the awareness of the present posthuman conditions, but it moves on nomadically towards the quest for sets of relations that will have constituted the time-continuum of becoming post human.

Braidotti presents a posthuman analysis that abstracts the syntropic in the WC ontogenetic Mamiwinini performance: The key notion in posthuman nomadic ethics is therefore the transcendence of negativity. What this means concretely is that the conditions for renewed political and ethical agency cannot be drawn from the immediate context or the current state of the terrain. They must be generated affirmatively and creatively by efforts geared to creating possible futures, by mobilizing resources and visions that have been left untapped and by actualizing them in daily practices of interconnection with others. This project requires more visionary power or prophetic energy, qualities which are neither especially in fashion in academic circles, nor highly valued scientifically in these times of coercive pursuit of globalized 'excellence'. Yes, the call for more vision is emerging from many quarters in critical theory.

WC created the Circle of All Nations to fray and fragment boundaries at every turn to enable contemporary global researchers to access the teachings of the Indigenous, who we have described as the Elder Sibling. In a world quite literally on fire, with no focus on the lens but only on the trigger, the concept of Ginawaydaganuc illuminates possibility.

As noted, the two motifs, Asinabka, and Wampum, are of great significant semiotic relevance in William Commanda's work. With geographical rootedness in earth and water, 
they constitute the anchors to ground his Anicinabe way of iteration and knowledge generation. The Circle of All Nations serves to keep his W ampum knowledge "encore vivant!" and emergent.

As Turnbull suggests:

"All processes of knowledge generation are based in the dynamics of movement through space, and of change over time, but how those dynamics are conceived, lived and represented vary between traditions, cultures and eras. In many of the western scientific traditions such spatiotemporal dynamics are largely rendered invisible by the ways that knowledge is conceived, in objectivist, representational terms, as abstracted and unified" (Turnbull 2007 141). Citing Rundstrom and Ingold, he adds, "From a performative perspective, the making of knowledge is simultaneously the making of space, and space is made by travelling [Rundstrom, 1990; 1991; 1993; 1995; Ingold, 2000]. Knowing is a form of travelling, of moving through space; and travelling, like knowledge, is also a form of narrative. ... With performativity, meaning, understanding, and knowledge are based in embodied practices", he adds, drawing on other researchers [Pickering, 1995; Nash, 2000; Thrift, 2000, 2004] (Turnbull 2007 142).

The question is to determine whether this relatively recent thinking in cartography can accommodate the conceptual views of Earth held by William Commanda and his ancestors, which evolved in the time/space/moving identity of Mamiwinini/Nomad, and also take root in the transformational interfaces he created for the Circle of All Nations operation in the cyber world. The preliminary analysis of indicators of ongoing interest in WC in the academy, his inclusion in historical monuments, his presence in social media and capacity to draw people to events suggests that his multi-faceted bridge building discourse has evolved in diverse webs of interface, and that cybercartography holds the potential to magnify this dispersal. Though none of this expression is actually managed or controlled, the core energy of his particular discourse appears to adhere and cohere as a viable entity. The growing interest from academics suggests that the pedagogical framework of his approach to knowledge generation may resonate for researchers in contemporary times. 


\subsection{A William Commanda Temporal Cyber Discourse Exemplar \\ A William Commanda Vision, Persistence and Presence}

The following images trace a few graphic moments in the evolving narrative of the Asinabka Sacred Chaudiere Site. They demonstrate the passionate interest of the thousands of people from across the globe who contributed to the development of this inclusive vision over the years. They also demonstrate the emergent discourse of Grandfather William Commanda, now animated by the diverse voices of his Circle of All Nations.

Year 2003

\section{Circle Vision \\ Sacred Asinabka - Chaudière/Victoria Island Site as endorsed by the Algonquin Elders and Communities of Quebec and Ontario in 2002-2003}

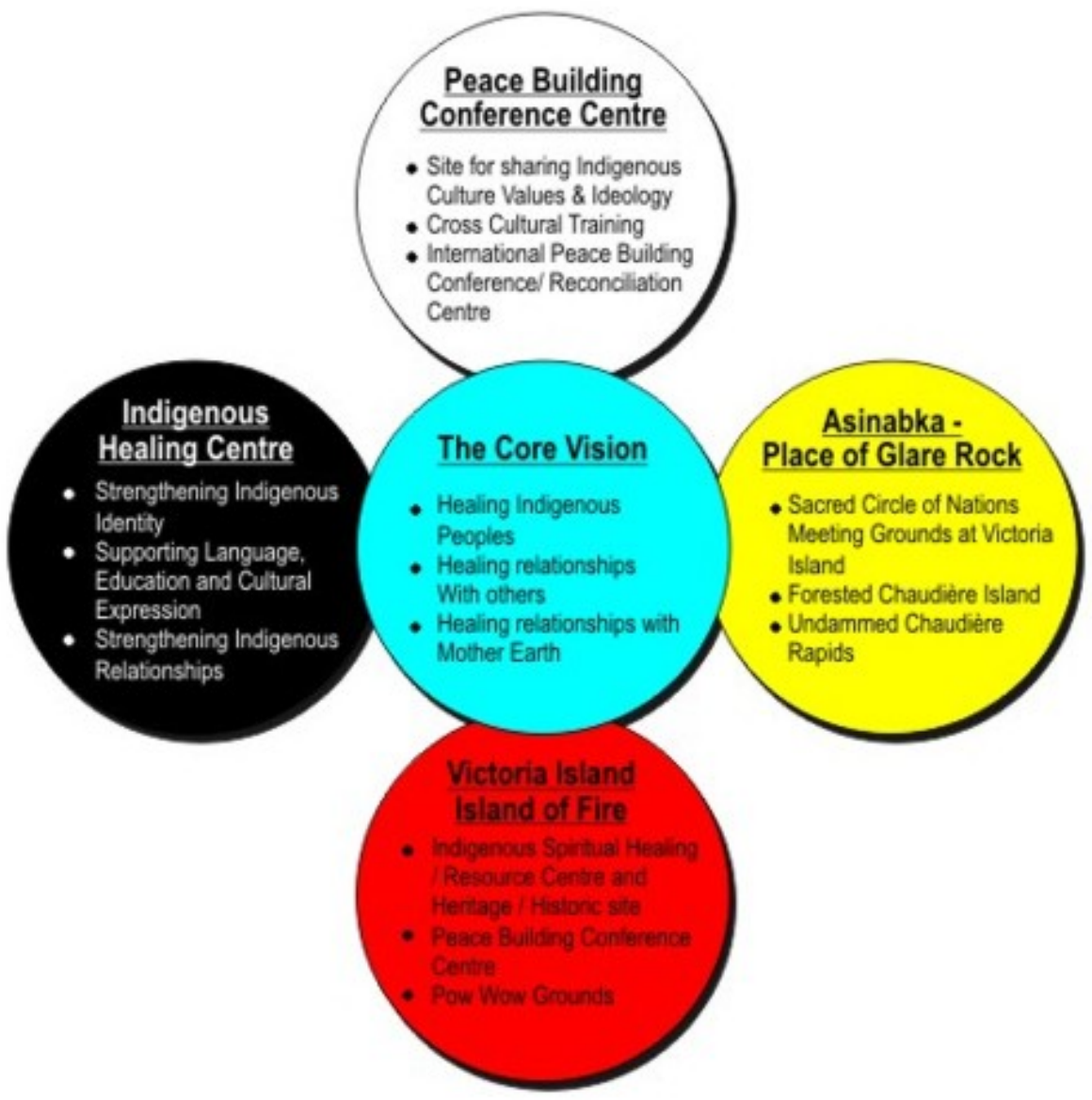

Figure 19. 2003 Circle Vision 
Year 2007

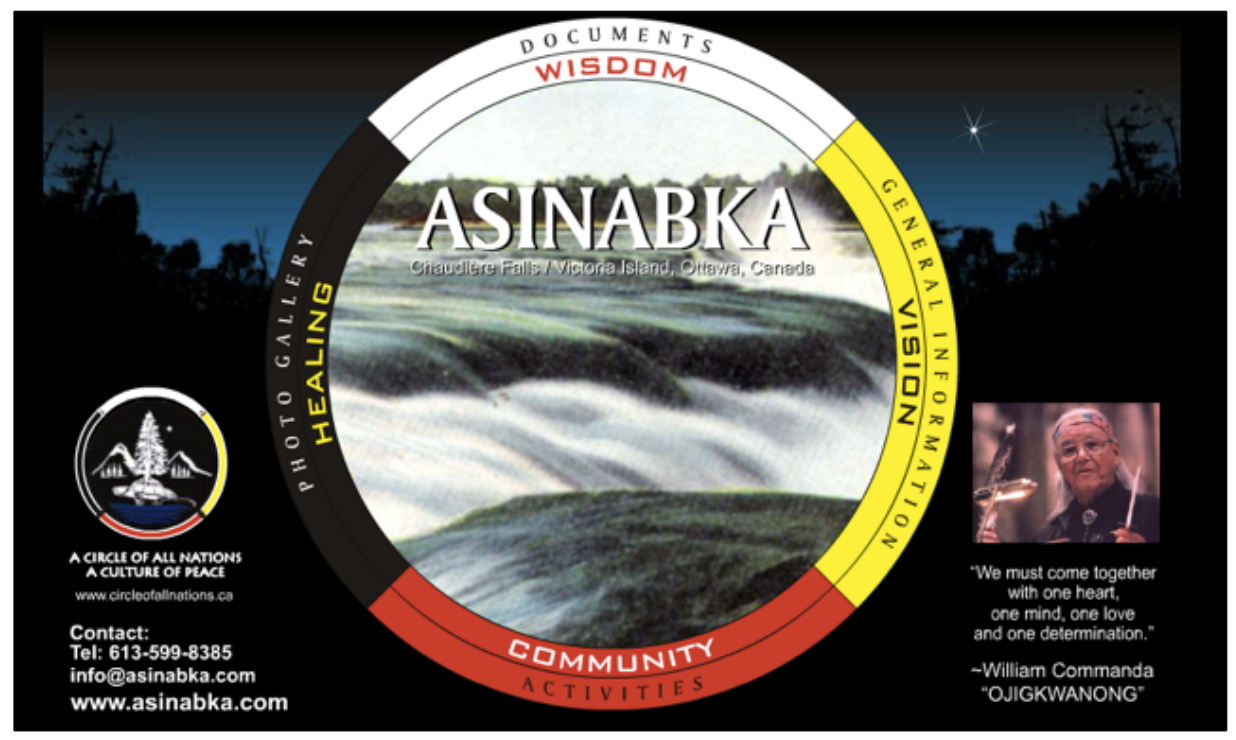

Figure 20. 2007 Asinabka Website

\section{Year 2008}

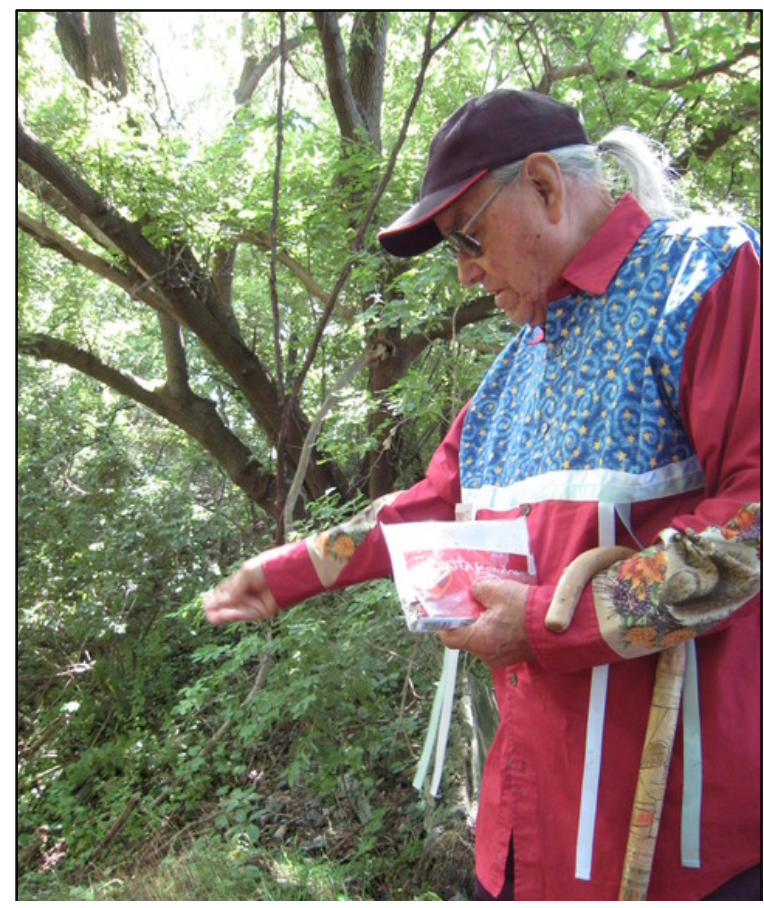

Figure 21. Grandfather's Prayer at the Chaudiere 


\section{Year 2008}

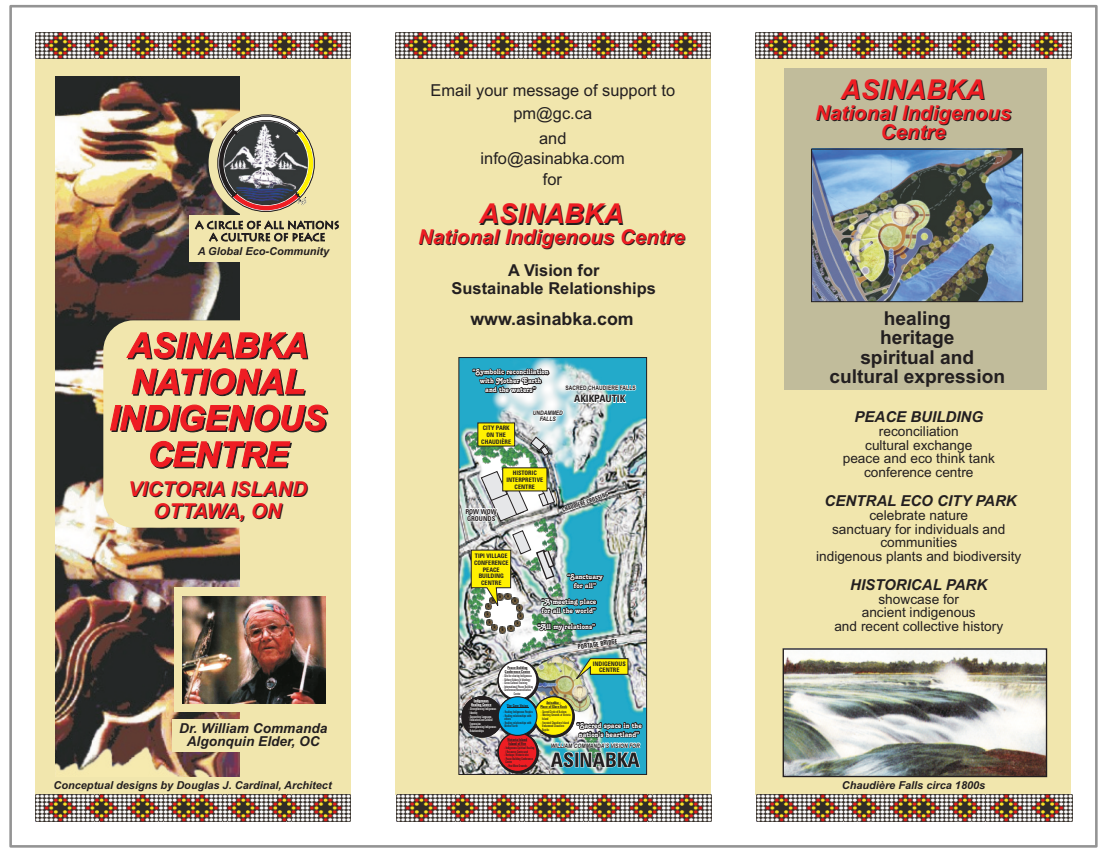

Figure 22. Asinabka National Indigenous Centre Brochure (English)

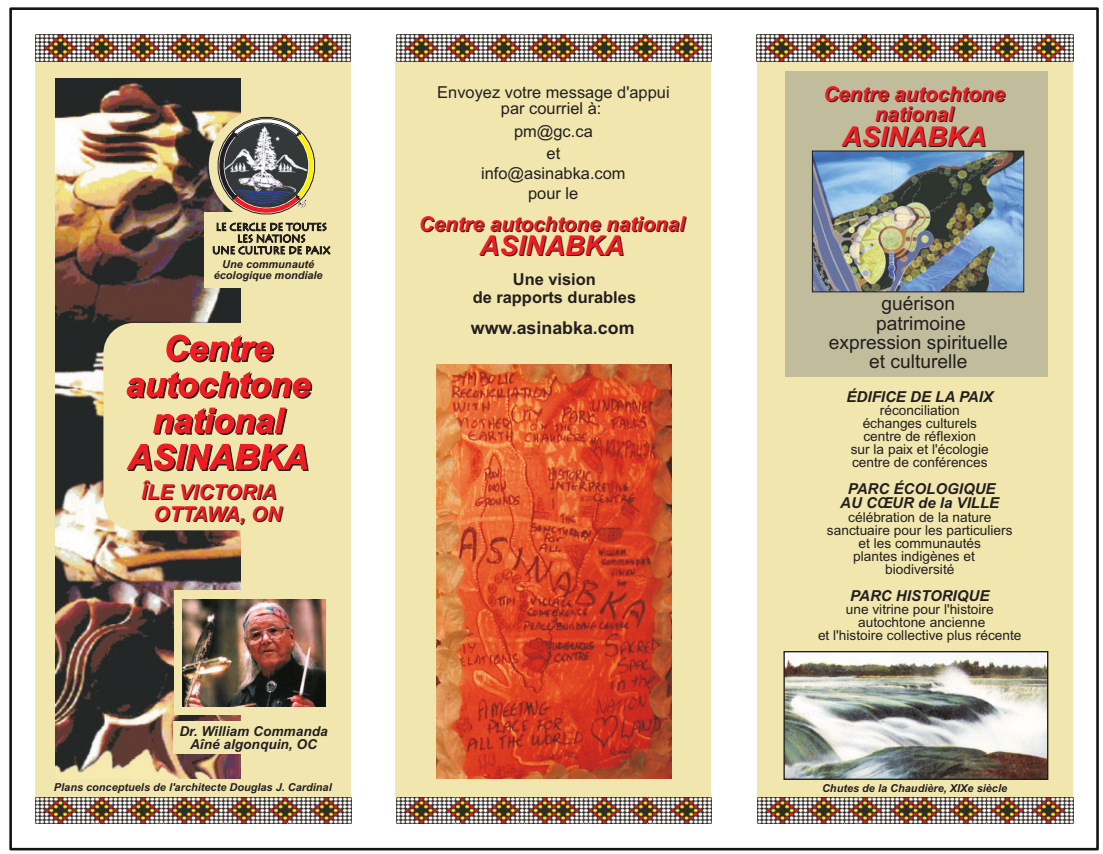

Figure 23. Asinabka National Indigenous Centre Brochure (French) 


\section{Year 2013 updated}

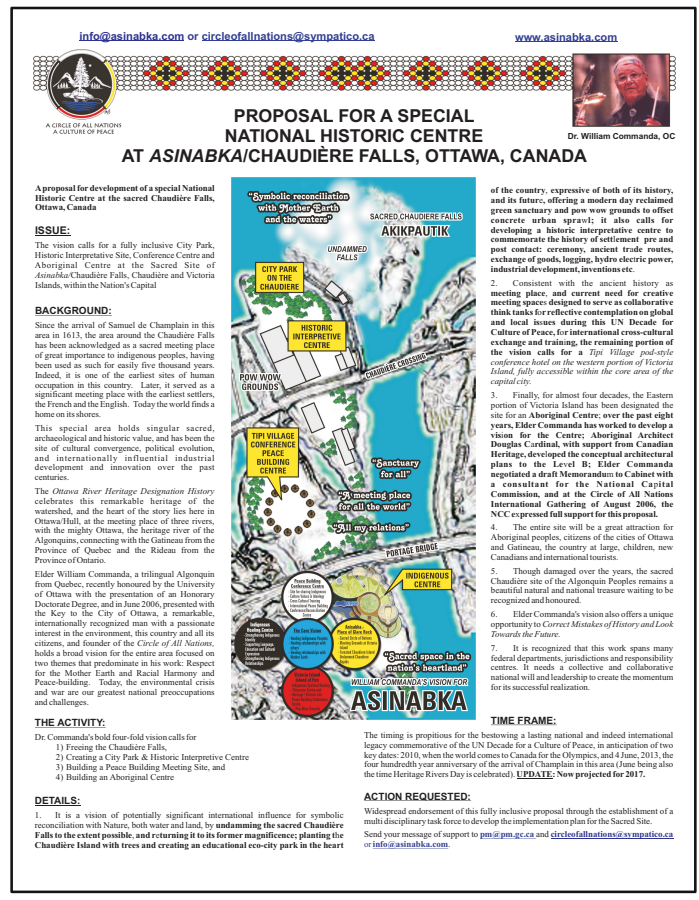

Figure 24. Asinabka National Indigenous Centre Brochure - Details (English)

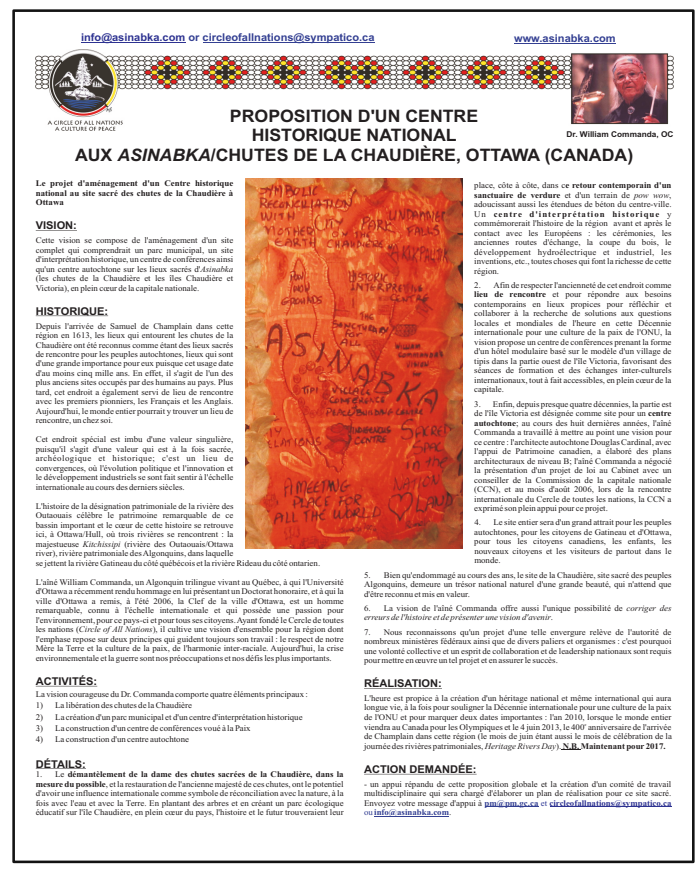

Figure 25. Asinabka National Indigenous Centre Brochure - Details (French) 


\section{Year 2015}

Romola Thumbadoo - Protecting the Sacred Chaudiere Falls Site in Ottawa Asinabka Sacred Chaudiere Site Timeline Development History

Video of Romola V. Thumbadoo's presentation on the Asinabka Vision at the 2014 Phil Ochs Festival, Ottawa, Ontario, produced by Greg Macdougall, EquitableEducation

Note also the William Commanda Legacy - Asinabka Facebook Page

\section{Year 2017}

Collage of the ongoing efforts to advance the William Commanda legacy vision for the Asinabka Sacred Chaudiere Site, created by Karen Bisson

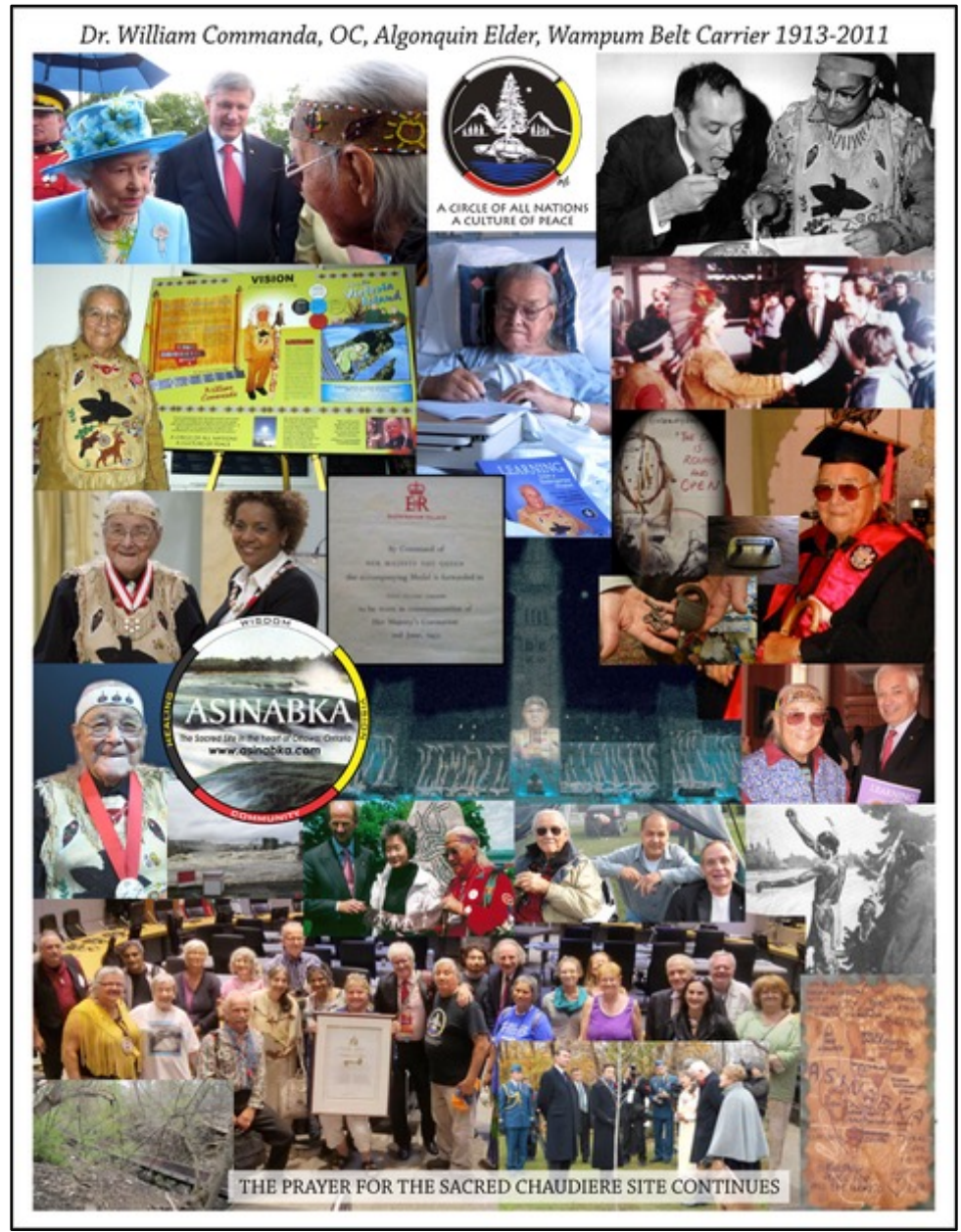

Figure 26. William Commanda Collage 


\section{Timeless}

Chief Commanda Dances at Asinabka Painting by Salvatore William

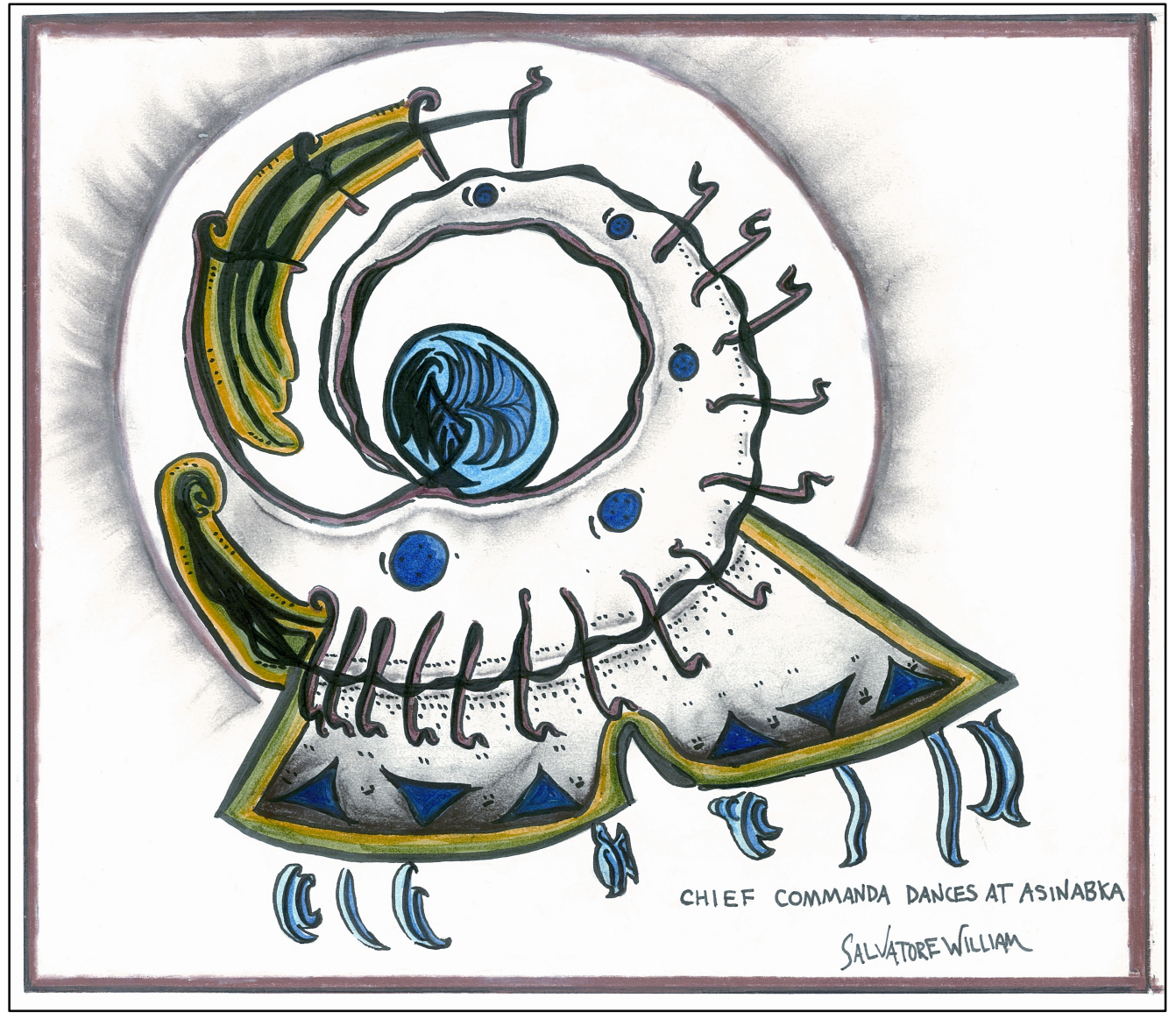

Figure 26. Artistic Depiction of William Commanda at Chaudiere 


\section{Chapter 8 Conclusion}

Situated within a turbulent confluence of performance, material and European mapping, Indigenous, physical, and human geography, and political market ecology and nascent environmentalism, WC persistently articulated a geography of relational spatiality and multi-directional temporality grounded in cosmic Laws of Nature, and ancient wampum doctrine.

By the nineteen fifties, Leopold (Sand CountyAlmanac: Sketches of Here and There 1949) and Carson (Silent Spring 1962) brought the growing environmental degradation in North America to the political foreground. DDT was banned, national parks were created and environmental monitoring agencies were established. By 1992, the UN mounted the Earth Summit, launched Agenda 21 and organized annual conferences to draw global attention to the need to manage the resources of the earth more judiciously. Emergent from an American heritage of managerial agricultural colleges, environmental sciences mushroomed into academic life. Meanwhile, environmental degradation accelerated, occurrence of environmental catastrophes escalated, climate change intensified, and extreme weather normalized.

From childhood, WC counted trucks loaded with trees decimating his North American homeland. He also noted the contaminated waters, poisoned earth, dying flora and fauna. Thirty years ago, in 1987, with wampum belts in hand publicly for the first time, he articulated his environmental warning on the national political stage, at the Aboriginal Constitutional Conference, drawing attention to the inseparable, intersectional, coconstituted materialization of Indigenous and environmental crises. In 1991, at the Pre-Rio Earth Conference in Paris, WC lit sacred fire to ignite a passion for global relational 
responsibility to Mother Earth. He also charted his own path to awaken people the looming crisis by hosting easily over a hundred earth-based gatherings grounded in his epistemology. He animated agentive activism at micro and macro levels in a spatial web spanning the local and global. Indigenous peoples across North America are reclaiming and restoring their languages, music and dance, spiritual traditions and ceremonies, responsibilities to Mother Earth, rivers and lands, social justice and healing practices, their ancestors and children, and WC's seeds are evident in every one of these movements. As anticipated by the Seven Fires Wampum Prophecy, Indigenous people have, without arms, resisted and challenged colonization and genocidal practices throughout the past century, have embarked on a robust program of reclaiming language, dance, culture, spirituality and relationship with land, are demanding and taking their place on the world stage, and are shifting global thinking in multiple ways. Despite disproportionate suffering in all colonized lands (land loss, social injustice, poverty, unemployment, racism, incarceration, health, substance abuse, suicide), they are no longer invisible. At the same time, the non-Indigenous peoples are growing increasingly aware of historical injustices and colonial privilege, as well as of the unique perspectives, strengths, sustainability and resilience of the knowledge systems of Indigenous Peoples. Many are now striving to connect and integrate with greater alliance, equality and respect.

Today, critical geographers and environmentalists are summoned to the battleground of an Anthropocentric world quite literally drowning in the Far East, on fire in the American North West, assaulted by hurricanes in the American North East, upturned by earthquake in Central America, undermined by melting ice and increase in icebergs in the North, fragmenting ice shelves in the South, and rising water levels, impacting winds and ocean currents, with desertification and food shortage decimating Africa, and plant and animal 
species departing Planet Earth in unprecedented numbers.

Many researchers now believe resilient Indigenous knowledge systems may hold keys for adaptation to the climate change, and researching Indigenous is becoming the new Renaissance for many. However, multiple challenges in bridging cultural divides persist.

I return to the research question of whether WC's thoughts on Mother Earth, environment and relational matters can contribute to greater integration of his approach in knowledge generation in academic discourse with mixed conclusions. I expect that it will be a challenge to integrate WC's approach to environment into the western academy. I turn to David N. Livingston's paper, The Polity of Nature: Representation, Virtue, Strategy (1995), where he writes that "representations of nature are implicated in moral evaluations which, in turn, have built into them incipient strategies for dealing with what are perceived to be environmental problems", the natural, cultural and moral being mutually constituted, and politics being associated with nature from 1760 (when Linnaeus, whose system of classification gave impetus to the seventeenth century Scientific Rationalism challenge of the control of religion over knowledge, presided over the dissertation of C. Daniel Wilke). Livingstone selected three dominant metaphorical representations of nature and tracked the virtues and strategies that flowed from them in a chain-linked fashion, in the context of environmental issues.

1. Representation of Nature as organism is associated with motherhood and materialized as weak and vulnerable feminine, to be approached by virtues of piety (and virginal) with environmental strategies aligned with mysticism and reverence; ramifications were the domination of nature/feminine, (as per Bacon's sanctioning of the exploitation of nature, interrogating and torturing it through mechanical devices, as witches, i.e. women, were, and Indigenous women now challenge).

2. Representation of Nature as clockwork and mechanical, such as was generated by the new heliocentric ideas of Copernicus and Galileo (ironically in my view likely triggered by the New World wholistic knowledge), and interpreted by Bacon, resulted in values convened around utility, and strategies aligned with her management; here, ramifications were "the shift from manorial farm economics to market capitalism, with its marked ecological consequences through windmills, 
watermills and taxation" (Livingstone 1995 356); ironically, law and justice asserted the dominion over nature theme, and were high-jacked to serve market capitalism.

3 Representation of Nature through association with Oikos, (a self-sufficient, coherent, stable, integrated community or unit) implicated in both ecology and also economy, led to values focused on community governed by politics. While some (like Leopold) awakened to biotic community and (like Muir) to preservation, others awakened to conservation, rooted in hierarchical political strategies that created the grounds for the rationalization of conservation to serve capitalism.

Twenty years after Livingstone's analysis, the consequences of each of these representations of nature have led to an acceleration towards the burnt and seared environmental conditions foreseen by the Seven Fires Prophecy.

For William Commanda,

Representation of Nature manifested via the elemental Laws of Nature, the associated values were respect, responsibility and reciprocity, and strategies advanced relationality, balance and equilibrium consistent with networked, interconnected, biospheric coherence, and syntropic renewal. The ramifications here are evolution, emergence and negentropic resurgence consistent with the laws of life. He affirmed total integration with a creative force which affirmed fertility, sexuality, fecundity, diversity and dynamism, where the feminine procreative energy, metaphoricized as Mother Earth, stimulated, seeded and fertilized by the Lighting and Thunders Winds, Rains from the masculinized Sky world, took care all her offspring, and also cleaned up after them.

This is why Indigenous peoples view the volcanoes, hurricanes and earthquakes marking this period of the Anthropocene as the time of the cleansing. WC's ancestors lived in sync with the rhythm of the changes of the natural cycle of evolution; today, we are in the throes of the ramifications of Man's choices, market capital man. From the moment he emerged on the global stage in the Pre-Earth Conference in France in 1990, when environmentalists, scientists and governments were seeking ways to manage the resources of the Earth more judiciously, WC was insisting on relationship with a living Mother Earth.

Livingstone concluded his critique with Elizabeth Bird's words: "We need histories of environmental problems that examine the social relations, structural conditions, cultural myths, metaphors, and ethical presuppositions that constitute the social negotiations with 
nature that contribute to these problems... What we should learn from these studies are the principles of social interaction with nature that will most likely prevent the recurrence of significant environmental degradation. What are the appropriate social expectations of nature? What are the stories we should tell? What are the ethics that should guide our negotiations?" (Bird in Livingstone 1995 371). He adds, "These are the questions ... that should remain high on our cultural and scientific agenda" (Livingstone 1995 371).

The Indigenous voice remains marginalized on the global stage; however, it is not invisible. Thousands have sought WC out over the years and still engage with his teachings in cyberspace. Who knows what lies ahead? It is interesting that Lovelock (Gaia hypothesis) describes the global system as the largest living organism and a "complex entity involving the Earth's biosphere, atmosphere, oceans and soil; the totality constituting a feedback or cybernetic system which seeks an optimal physical and chemical environment for life on earth" (Lovelock in Livingstone 1995 358). This cybernetic loop represents a massive closed system, yet, paradoxically, it is part of the galactic open system that can only be comprehended over deep time and deep space. It is this interface that WC accessed via his Laws of Nature. Cybercartography and digital media now permit the accessing and mainstreaming of an infinite number of innovative, evolving and spiraling Indigenous knowledge loops that were once deliberately obscured, appropriated, misunderstood and marginalized to serve and guide a communal global future.

WC and I used to joke that we would call his next book, Believe it or Else. Perhaps relational interface with his pedagogical tools can facilitate integration of Indigenous approaches in knowledge generation on environment and related matters.

\section{Concluding Remarks}

Over the course of the past five years, the thesis research has been impacted by two 
tangential responsibilities, one pertaining to the land-based challenges at the geo-cultural historical heritage site identified by WC for his global negentropic project for these times of unprecedented environmental crisis and human conflict, and the other the continuation of his legacy Circle of All Nations work, without its usual land-based supports. These challenges have each required triangulated focalization on the future and the present, with the thesis research examining the past. While it has been easier to analyse WC's discourse, and abstract critical elements of agency and praxis, it has been extremely challenging to translate the infinitude of orality and narrativity into the abstractive language of the academy, to negotiate perspectives of subjectivity and objectivity simultaneously, articulate them with finesse, to serve diverse audiences, including Indigenous and non-Indigenous academics.

It is also a challenge to share this cross-cultural educational project spiraling around Indigenous knowledge because it runs the tremendous risk of robotinizing and fixing that which is fluid and re-generative. The thread of interface is fragile and extremely vulnerable to manipulation, appropriation and misappropriation and yet it is also resilient and resistant. However, with too much resistance it can become brittle; too much external structure and it fragments and deflects its integrity. Yet in the challenging contemporary world of the Anthropocene, WC (and countless other ancient Indigenous voices) have been re-emerging in the global consciousness to point to a co-created trail that may offer insights for knowledge generation. However, the centrality of Indigenous is critical. I liken WC's work to that of Earthworm. He focused on aerating soil that has become compacted and deoxygenated, in identifying and combating pollutants, ingesting and transforming contaminants, and regurgitating new soil. This soil can support the sprouting of seeds with potential for anchoring into a Mother Earth already now embarked on a new evolutionary spiral. How we navigate in the new spatial domain will in part be determined by how well 
we access the multi-directional temporal signifiers of Indigenous prophecy, animate the genius of the fundamental message of generosity, integrate the spirit of Ginawaydaganuc in deeply personal and communal experiential ways, and activate the laws of syntropy.

The animation of the spirit of Ginawaydaganuc served WC as guiding principle to navigate shifting social and environmental landscapes in the zone of borderlessness of his eco-peace Circle of All Nations community, consistent with the mandate of the Three Figure Welcome Wampum Belt, and the Border Crossing Wampum Belt. Metaphorically, WC integrated the fourth and Missing Wampum Belt as the impetus for negentropy, and the emergence of new cybernetic circuits of knowledge. I have presented the logics of Ginawaydaganuc and syntropy to interrogate the confluence, and examined conceptual tools of the Circle of All Nations medicine wheel to draw the boundlessness of the oral into more accessible quarters for accessing by those interested in understanding WC's approach to human and environmental issues better.

My conclusion as geographer is that WC's ontology was informed by cartography and physical geography, his global outreach and ontogenesis was driven by human geography and environmental crises, that he animated an epistemology grounded in politics, humanities and Laws of Nature in his Circle of All Nations work, and that his negentropic legacy vision for the Asinabka Sacred Chandiere Site was his prayer for a collective global future. His knowledge was accessed via his orality and narrative, and the motional way of life of Mamiwinini, which triggered Ginawaydaganuc relationality. Future research will examine the implications of William Commanda's oral storytelling and body-in-motion indoctrination in greater depth, to track human relational interface with the laws of nature and contemporary planetary changes; and also to determine the nature of Circle of All Nations discursive elements discernible in the contemporary environmental movement and in the 
cybercartographic world. The cosmic medicine wheel continues spiraling, the journey continues, and his syntropic answers to the research question emerge, in his Dance Across the Universe, Dance Across the Seas of Time:

Johnny Clegg \& Savuka - Dance Across The Centuries - YouTube 3:54 ${ }^{9}$

9 Johnny Clegg: Dance Across The Centuries

It's a world of "hang on to what you've got"

It's a world of "do or die"

I know you never take it serious

And you keep your head up high

I know you'd like to speak to the Manager

Oh baby, so would I

I never made this crazy world anyway

I never made this one way ride

Chorus For some life offers nothing more Than a struggle or a war A magnum hidden in your drawer You walk away... you want to Dance across the centuries Dance across the sea of time Dance a living memory

oh Dance you want to let your spirit shine

Every dog wants to be a fat cat

Every mouse to be a dog

It's not easy, I can tell you

Trying to see through the fog

Funny how some of us live it up

While others just survive I know you do what you can

But all you ever do is time. Chorus

Yaguka lenkunzi emnyama (The black bull has grown old)

Kade ihlaba ithetha amalala (Long and well it has fought and argued)

Yaguka lenkunzi emnyama (The black bull has grown old)

Guka 'sithembe kade wawudlelwa (My gating mat grows old, well has it served me)

(All things must pass and change). Chorus 


\section{Bibliography}

Abrahams, P. (2000). The Coyaba Chronicles: Reflections on the Black Experience in the 20th Century. Cape Town: David Philips.

Adams, C. J. (1995). Neither Man nor Beast: Feminism and the Defense of Animals. New York: Continuum.

Adkins, L. and Lury, C., (Eds.). (2012). Measure and Value. Chichester, UK: Wiley-Blackwell.

Aitken, S. and Valentine, G. (2006). Approaches to Human Geography. London: Sage Publication Ltd.

Alfred, T. (2008). Opening Words. In Simpson, L. Ed., Lighting the Eight Fire: The Liberation, Resurgence, and Protection of Indigenous Nations. Winnipeg, Manitoba: Arbeiter Ring Publishing, 9-11.

Allen, W. (2005). Report to Ojigkwanong Elder William Commanda. October 7, 2005 Flight to Catfish Lake, Algonquin Park. William Commanda's Archives.

Andersen, N. (2010). Exemplars in Environmental Ethics: Taking Seriously the Lives of Thoreau, Leopold, Dillard and Abbey. Ethics Place and Environment, 13(1), 43-55.

Andersen, R. R. and Crellin J. K. (2009). Mi'sel Joe An Aboriginal Chief's Journey. St John's, Newfoundland: Flanker's Press Ltd.

Anderson, K. (1999). Reflections on Redfern, in Stratford, E. (Ed.) Australian Cultural Geographies. Melbourne: Oxford University Press.

Archibald, L., \& Crnkovich, M. (1995). Intimate Outsider: Feminist Research in a CrossCultural Environment, in S. Burt, \& L. Code (Eds.). Changing methods: Feminists transforming practice. 105-126.

Awan, N. (2016). Introduction to Border Topologies. GeoHumanities, 2(2), 279-283.

Bai, H. (2004). The Three I's for Ethics as an Everyday Activity: Integration, Intrinsic Valuing, and Intersubjectivity. Canadian Journal of Environmental Education, 9, 51-63.

Baker, J. (2002). Production and Consumption of Wilderness in Algonquin Park. Space and Culture, 5(3), 198-210.

Baldwin, A. (2009). Ethnoscaping Canada's Boreal Forest: Liberal Whiteness and its Disaffiliation from Colonial Space. The Canadian Geographer, 53(4), 42-443.

Barnett, H. (1999). The Land Ethic and Environmental Crime. Criminal Justice Policy Review, 10(2), 161-191. 
Barrett, M. (1991). The Politics of Truth: From Marx to Foucault. Stanford, California: Stanford University Press.

Battiste, M. and Henderson, J. S. Y. (2000). Protecting Indigenous Knowledge and Heritage. Saskatoon, Saskatchewan: Purich.

Bauder, H. (2006). Learning to Become a Geographer: Reproduction and Transformation in Academia. Antipode, 38(4), 671.

Beatley, T. (1989). Environmental Ethics and Planning Theory. Journal of Planning Literature, $4(1), 1-32$.

Belshaw, C. (2001). Environmental Philosophy: Reason, Nature and Human Concern. Montreal; McGill-Queen's University Press.

Berg, L. D. (1993) Between Modernism and Postmodernism. Progress in Human Geography, 17, 490-507.

Berg, L. D. (1994b). Masculinism, Power and Discourse of Exclusion in Brian Berry's "Scientific Geography. Urban Geography, 15, 279-87.

Berg, L. D., and Kearns, R.A. (1998). America Unlimited. Environment and Planning D: Society and Space, 16, 128-32.

Berg, L. D. and Mansvelt, J. (2000). Writing In, Speaking Out: Communicating Qualitative Research Findings. In Hay (Ed.), Qualitative Research Methods in Human Geography. Melbourne: Oxford University Press.

Berque, A. (2005). A Basis for Environmental Ethics. Diogenes, 52(207), 3-12.

Bieman, U. (2016) Deep Weather in Forum on Border Topologies: Practices and Curations, GeoHumanities, 2 (2), American Association of Geographers Taylor and Francis Group, LLC, 373-376

Bird, E. A. R., (1987). The Social Construction of Nature: Theoretical Approaches to the History of Environmental Problems. Environmental Review, 11, 255-264.

Blomley, N. (2008). The Spaces of Critical Geography. Progress in Human Geography, 32(2), 285-293.

Bondi, L. 1997. In Whose Words? On Gender Identities, Knowledge and Writing Practices. Transactions of the Institute of British Geographers, 22, 245-58.

Bordo, S. (1986). The Cartesian Masculinization of Thought. Signs 11, 439-456.

Bookchin, M. (1980). Toward an Ecological Society. Montreal: Black Rose Books. 
Bradshaw, M. and Williams, S. (1999). Scales, Lines and Minor Geographies: Whither King Island. Australian Geographies Studies, 37(3), 248-267.

Braidotti, R. (2016). Posthuman Critical Theory. In Banerji, D. Paranjape, M.R. (Eds.), Critical Posthumanism and Planetary Futures. http:/ /www.springer.com/978-81-81-322-3635-1

Bratton, S. (2004). Thinking Like a Mackerel: Rachel Carson's Under the Sea-wind as a Source for a Trans-ecotonal Sea Ethic. Ethics \& the Environment, 9(1),1-22.

Braun, B. (1996) Materializing Nature: Discourse, Practice and Power in the Temperate Rainforest. PhD Dissertation, University of British Columbia.

Braun, B. and Wainwright, J. (2001) Nature, Poststructuralism, and Politics. Social Nature: Theory, Practice, and Politics. N. Castree and Braun (Eds.), Oxford: Blackwell, 41-63.

Brennan, A., \& Lo, Y. (2008/2011). Environmental Ethics. In The Stanford Encyclopedia of Philosophy. Retrieved from http://plato.stanford.edu/entries/ethics-environmental/

Brodie, J. (2007). Reforming Social Justice in Neoliberal Times. Studies in Social Justice, 1(2), 93-107.

Butler, J. (1990). Gender Trouble: Feminism and the Subversion of Identity. London and New York: Routledge.

Burger, J. (1990). The Gaia Atlas of First Peoples. London: Anchor Books Doubleday.

Callicott, J. B. (1999). Beyond the Land Ethic: More Essays in Environmental Philosophy. Albany, N.Y: State University of New York Press.

Cannavò, P. F. (2012). Ecological Citizenship, Time, and Corruption: Aldo Leopold's Green Republicanism. Environmental Politics, 21(6), 864-881.

Caquard, S. (2011), Cartography 1: Mapping Narrative Cartography. Progress in Human Geography, 37(1), 135-144.

Caquard, S. Pyne, S., Igloliorte, H., Mierins, K., Hayes, A. and Taylor, D.R.F. (2009). A Living Atlas for Geospatial Storytelling: The Cybercartographic Atlas of Indigenous Perspectives and Knowledge of the Great Lakes Region, Cartographica, 2-15.

Carson, R. (1994). Silent Spring. Boston: Houghton Mifflin.

Casti, E., Taylor, D. R. Fraser, Series Editor. (2015). Reflexive Cartography: A New Perspective on Mapping. Amsterdam: Elsevier.

Castree, N. (1995). The Nature of Produced Nature: Materiality and Knowledge Construction in Marxism. Antipode, 27(1), 12-28. 
Castree, N. (2003a). A Post-environmental Ethics? Ethics, Place \& Environment, 6(1), 3-12.

Castree, N. (2003b). Environmental Issues: Relational Ontologies and Hybrid Politics.

Progress in Human Geography, 27(2), 203-211.

Castree, N. (2005). Nature. New York, NY: Routledge.

Champlain, S. de. (2009). The Voyages and Explorations of Samuel de Champlain (1604-1616) Two

Vols. La Vergne, Tennessee: Bibliobazaar.

Chase, S. (2005). Narrative Inquiry: Multiple Lenses, Approaches, Voices. In N. K. Denzin, \& Y. S. Lincoln (Eds.), The Sage handbook of qualitative research (3rd ed.) Thousand Oaks: Sage Publications, 651-680.

Chatterton, P. (2008). Demand the Possible. Antipode, 40(3), 421-427.

Chatwin, B. (1987). The Songlines. New York: Penguin Books.

Chua, A. (2003). World on Fire. New York: Anchor Books.

Churchill, W. (2002). Struggle for the Land: Native North American Resistance to Genocide, Ecocide, and Colonization. San Francisco: City Lights.

Commanda, W. (1999). Wampum Teachings at Prayer Vigil in Washington DC Video. Woodstock, New York: Wittenberg Centre.

https://www.youtube.com/watch?v=h0Vs8OmxLcA

Commanda, W. (2005). Video Interview on the UN International Day of Peace. Trudel, J. Montreal, Quebec: Cercle de Paix https://youtu.be/zmYs2PtPc4w

Commanda, W. (2011). In Chief, K. Audio Recording/Song Miskojonia Red Gold (Flying

Down Thunder and Rise Ashen One Nation 2011 YouTube

Commanda, W. (2009). Audio Interview: Asinabka Sacred Chaudiere Site and American Eel. http://www.asinabka.com/MP3s/William\%20Commanda \%20Re\%20Land, $\% 20$ Eels, $\% 20 \mathrm{Pr}$ ophecy, $\% 20$ Indig $\% 20$ Centre.mp3

Concordia Centre for Oral History and Digital Storytelling. (2009). General interview guidelines for CURA life stories project. Retrieved 03/01, 2013, from http://storytelling.concordia.ca/oralhistory/resources/tips/Documents/InterviewGuidelines-April2009.pdf

Cook, I., et al. (2007). It's More Than Just What It Is: Defetishising Commodities, Expanding Fields, Mobilizing Change... Geoforum 38, 1113-26.

Clement, D. Ed. (1996). The Algonquins. Hull, Quebec: Canadian Museum of Civilization. 
Crampton, J. W., (2001, Maps as Social Constructions: Power, Communications and Visualization. Progress in Human Geography, 25(2), 235-252.

Crampton, J.W. and Elden, S. (Ed.) (2007). Space, Knowledge and Power: Foucault and Geography. Ashgate Publishing/Amazon.com.

Crampton, J. W., (2009). Cartography: Mapping 2.0. Progress in Human Geography, 33(1), 91 100.

Cresswell, T. (2009). Discourse. 2009 International Encyclopaedia of human geography: Oxford: Elsevier, $211-214$.

Cresswell, T. (2004). Place: A Short Introduction. Malden, MA: Blackwell.

Cronon, W. (1995a). The Trouble with Wilderness; Or, Getting Back to the Wrong Nature. In Cronon, W. (Ed.) Uncommon Ground: Rethinking the Place in Nature. New York, NY: W.W. Norton, 69-90.

Cronon, W. (Ed.). (1995b). Uncommon ground: Toward Reinventing Nature (1st ed.). New York, NY: W.W. Norton.

Davis, W. (2009). The Wayfinders: CBC Massey Lectures. Toronto: House of Anansi Press Inc.

de Lotbiniere, P. J. 1996. Of Wampum and Little People: Historical Narratives Regarding the Algonquin Wampum Record. In Clement, D. (Ed.) (1996). The Algonquins. Hull, Quebec: Canadian Museum of Civilization, 93-121.

Dempsey, J. (2010). Tracking Grizzly Bears in British Columbia’s Environmental Politics. Environment and Planning A, 42(5), 1138-1156.

Del Casino Jr., V.J. and Hanna, S.P. (2006). Beyond the 'Binaries': A Methodological Interventions for Interrogating Maps as Representational Practices. Acme: An International EJournal for Critical Geographies, 4(1), 34-56.

Denzin, N.K., Lincoln, Y.S. and Tuhiwai Smith, L. (2008). Handbook of Critical and Indigenous Methodologies. Thousand Oaks California: Sage.

Derrida, J. (1981). Dissemination. Tr. Johnson, B. Chicago: University of Chicago Press.

Di Corpo, U. and Vannini, A. (2001). Origin of Life, Evolution and Consciousness in the Light of the Law of Syntropy. https://www.Amazon.com: Ulisse Di Corpo Kindle Book.

Di Leo Browne, T., Ljubicic, G. (2014). Considerations for Informed Consent in the Context of Online, Interactive, Atlas Creation. In Taylor, D. F. R., Ed., and Lauriault, T. Assoc. Ed. (2014). Developments in the Theory and Practice of Cybercartography: Applications and Indigenous Mapping. Amsterdam: Elsevier, 263-278. 
de Certeau, M. (2000). Making do: Uses and Tactics. In The Consumer Society Reader, Lee, M.J. (Ed.) Berkley: University of California Press, 162-174.

Deleuze, G. and Guattari, F. (1987). A Thousand Plateaus: Capitalism and Schizophrenia. Tr. Massumi, B. London, MN: University of Minneapolis Press.

Dowling, R. (2010) Power, Subjectivity, and Ethics in Qualitative Research. In Hay, I. (Ed.) Qualitative Research Methods in Human Geography. Don Mills, On: Oxford University Press, 2639.

Derrida, J. (1993). The Animal that Therefore I am (More to Follow). Tr. Wills, D. Critical Inquiry 28 (Winter), 369-418.

Durant, J. (1944). The Best Guide in the World Article on Alonzo Commanda. New York: Saturday Evening Post, December 23, 1944. (Copy in William Commanda's archives).

Emoto, M. (2002). Messages from W ater. Japan: Hado Kyoikusha Co., Ltd.

England, K.V.L. (1994). Getting Personal: Reflexivity, Positionality, and Feminist Research. Professional Geographer, 46,80-9.

Erickson, B. (2008). Canoe Nation: Canoes and the Shifting Production of Space Through White Canadian Masculinities. Antipode, 40(1), 182-184.

Escobar, A. (1995). Encountering Development. Princeton: Princeton University Press.

Escobar, A. (2008). Territories of Difference: place, movements, life. Durham and London: Duke University Press.

Escobar, A. (2010). Postconstructivist Political Ecologies. The International Handbook of Environmental Sociology. Redclift, M.R. and Woodgate, G. (Eds.). Cheltenham: Edward Elgar Publishing Ltd, 91-105.

Evanoff, R. (2007). Bioregionalism and Cross-cultural Dialogue on a Land Ethic. Ethics, Place \& Environment, 10(2),141-156.

Evans, M., Hole, R., Berg, L. D., Hutchinson, P., \& Sookraj, D. (2009). Common Insights, Differing Methodologies Toward a Fusion of Indigenous Methodologies, Participatory Action Research, and White Studies in an Urban Aboriginal Research Agenda. Qualitative Inquiry, 15(5), 893-910.

Fanon, F. (1963). The Wretched of the Earth. New York: Grove Press.

Farbotko, C., \& McGregor, H. V. (2010). Copenhagen, climate science and the emotional geographies of climate change. Australian Geographer, 41(2), 159-166.

Ferguson, J. (2003). L'Algonquin Gabriel Commandant. Sillery, Quebec: Septentrion. 
Flowerdew, R. and Martin, D. (Eds). (2005). Methods in Human Geography - A Guide for Students Doing a Research Project - Second Edition Harlow: Longman.

Flores, T. (2015). Beyond Centre-periphery: Modernism in Latin American Art. In Lindgren, A. and Ross, S. (Eds) (2015). The Modernist World. London and New York: Routledge. 426436.

Flyvbjerg, B. (2001). Making Social Science Matter. New York, USA: Cambridge University Press.

Fox, W., (1990). Toward a Transpersonal Ecology : Developing New Foundations for Environmentalism ( $1^{\text {st }}$ Ed.) Boston: Random House.

Foucault, M. (1981). The Order of Discourse. In Young, R. (Ed.) Untying the Text: A Poststructuralist Reader. London: Routledge, 48-79.

Foucault, M. (1977b) Language, Counter-memory, Practice: Selected Essays and Interviews. Ithaca: Cornell University Press.

Foucault, M., (1972). The Archaeology of Knowledge. London: Tavistock Publications Ltd.

Foucault, M., (1973). The Order of Things. New York: Vintage Books Edition.

Freire, Paulo (1970). Pedagogy of the Oppressed. New York: Continuum International Publishing.

Fullagar, S., (2009) Nature, Performig. Kitchen, R. and Thrift, N. Eds. In International encyclopedia of human geography. Oxford: Elsevier, 298-302.

Gardiner H., (1993) Multiple Intelligences. New York: Basic Books: Harper Collins.

Gasking, D. A. T. and A. C. Jackson. (1967). Wittgenstein as a Teacher. Ludwig Wittgenstein: the Man and His Philosophy. Fann, K.T. (Ed.). Sussex: Harvester Press.

Gehl, L. (2014). Mkadengwe: Sharing Canada's Colonial Process Through Black Face Methodology. Peterborough, Ontario: Algonquin Anishinaabe-Kwe Publishing.

Gehl, L. (2014). The Truth that Wampum Tells. Black Point, Nova Scotia: Fernwood Publishing.

Gehl, L. (2017). Claiming Anishinaabe: Decolonizing the Human Spirit. Regina, Saskatchewan: University of Regina Press.

Geiger, S. (1986). Women's Life Histories: Method and Content. Signs, 11(20), 334-351.

Geiger, S. (1992). What's So Feminist About Doing Women's Oral History. In C. JohnsonOdim, \& M. Strobel (Eds.), Expanding the Boundaries of Women's history. 305-318.

Geertz, Clifford. (1973). The Interpretation of Cultures. New York: Basic Books. 
George, K., \& Stratford, E. (2010). Oral History and Human Geography. In Hay, I. (Ed.), Qualitative Research Methods in Human Geography (Third ed.,). Don Mills, Ontario: Oxford University Press.

Gibson-Graham, J. K. (2006). A Postcapitalist Politics. Minneapolis: University of Minnesota Press.

Gilchrist, K. (2010). "Newsworthy” Victims? Feminist Media Studies, 10(4), 373-390.

Graham, E. (1997). Philosophies Underlying Human Geography Research. In Flowerdew R. and Martin, D. (Eds.), Methods in Human Geography: A guide for Students Doing Reaearch Projects. Edinburgh: Longman. 6-30.

Grant, G. (1995). Lament for a Nation. Ottawa, Ontario: Carleton University Press.

Gréber, J. (1950). Plan for the National Capital: General Report Submitted to the National Capital Planning Committee. Ottawa: National Capital Planning Service. Online. https://qshare.queensu.ca/Users01/gordond/planningcanadascapital/greber1950/ Full_text_Greber1950.pdf.

Grimwood, B. S. R. (2011). Thinking Outside the Gunnels: Considering Natures and Terrains of Recreational Canoe Travel. Leisure/Loisir 35(1). Canada: Routledge Taylor and Francis Group, 49-69.

Haigh, M. (1996). Empowerment, Ethics, Environmental Action: A Practical Exercise. Journal of Geography in Higher Education, 20(3), 399-411.

Hall, C.M., \& Page, S.J. (2006). The Geography of Tourism and Recreation: Environment, Place and Space (3rd ed.). New York, NY: Routledge.

Haraway, D. (1991). Situated Knowledges: The Science Question in Feminism and the Privilege of Partial Perspective. In Haraway, D., Ed., Simians, Cyborgs and Women: The Reinvention of Nature. London: Routledge.

Haraway, D. (1992). The Promises of Monsters: A Regenerative Politics for Inappropriate/d Others. In Cultural Studies. Barnes, T. and Duncan, J. (Eds.) London: Routledge, 231-247.

Harley, J.B., (1989). Deconstructing the Map. Cartographica Vol 26 No 2 Summer 1989, 1 - 20.

Harris, L. and Harrower, M. (2006). Introduction Critical Interventions and Lingering Concerns: Critical Cartography/GISci, Social Theory, and Alternative Possible Futures. Acme: An International E-Journal for Critical Geographies, 4 (1), 1-10.

Harvey, D. (2005). A Brief History of Neoliberalism. Oxford: Oxford University Press.

Haun-Moss, B. (2002). Layered Hegemonies: The Origins of Recreational Canoeing Desire in the Province of Ontario. Topia: Canadian Journal of Cultural Studies, 7, 39-55. 
Hay, I. (2010). Qualitative Research Methods in Human Geography. Don Mills, Ontario: Oxford University Press.

Hayes, A., Pulsifer, P. L., Fiset, J. P. (2014). The Nunaliit Cybercartographic Atlas Framework. In Taylor, D. F. R., (Ed.), and Lauriault, T. (Assoc. Ed.) Developments in the Theory and Practice of Cybercartography: Applications and Indigenous Mapping. Amsterdam: Elsevier, 129140.

Henry, M., and Berg, L.D. (2006). Geographers Performing Nationalism and Heteromasculinity. Gender, Place and Culture, 13 (6), 629-645.

Hessel, P. D. K. (1987). The Algonkin Tribe. Arnprior, Ontario: Brittle Printing Ltd.

Hinchliffe, S. (2007). Geographies of Nature: Societies, Environments, Ecologies. Thousand Oaks, CA: Sage.

Hodgins, B. (1988). Canoe Irony: Symbol and Harbinger. In J. Raffan \& B. Horwood (Eds.), Canexus: The Canoe in Canadian Culture . Toronto, Ontario: Betelguese Books, 45-57.

Holifield, R. (2009). Actor-network Theory as a Critical Approach to Environmental Justice: A Case Against Synthesis with Urban Political Ecology. Antipode, 41(4), 637.

Holt-Jensen, A. (2009). Geography : History and Concepts : A Student's Guide (4th ed.) Los Angeles: Sage.

Howitt, R., Muller, S., \& Suchet-Pearson, S. (2009). Indigenous Geographies. In Kitchin, R. \& Thrift, N. (Eds.), International encyclopedia of human geography. Oxford: Elsevier, 358-364.

Hoy, D. C. Ed., (1986). Foucault A Critical Reader. Massachusetts: Blackwell Publishers Inc.

Hull, R. (2005). All About EVE: A Report on Environmental Virtue Ethics Today. Ethics and Environment. 10 (1), 89-110.

Jay, N. (1981). Gender and Dichotomy. Feminist Studies, 738-56.

Jessop, B. (1990). Putting States in their Place: Once More on Capitalist States and Capitalist Societies. In Jessop, B. State Theory. Pennsylvania: Penn State University, 338-369.

Jessop, B. (2009). State Theory. Oxford: Elsevier, 1-6.

Jickling, B. (2005). Ethics Research in Environmental Education. South African Journal of Environmental Education, 22, 20-34.

Jóhannesson, G. T., \& Bærenholdt, J. O. (2009). Actor-network Theory/Network Geographies. In Kitchin, R. \& Thrift, N. (Eds.), International Encyclopedia of Human Geography, Oxford: Elsevier, 15-19.

Johnson, J.T., Louis, R.P., and Pramono, A.H., (2009). Facing the Future: Encouraging 
Critical Cartographic Literacies In Indigenous Communities. Acme: An International E-Journal for Critical Geographies, 4 (1), 80-98.

Jones, O. (2009). Nature-culture. In Editors-in-Chief: Rob Kitchin, \& Nigel Thrift (Eds.), International Encyclopedia of Human Geography. Oxford: Elsevier, 309-323.

Julien, E. (2006). The Kogi, Message from the Last Man. Video Documentary.

www.laboutique.gedeonprogrammes.com

Kaldis, B. (2003). Could the Environment Acquire its Own Discourse? History of the Human Sciences, 16(3), 73-103.

Keith, D., Crockatt, K., Hayes, A. (2014). The Kitikmeot Place Names Atlas, in Taylor, D. F. R., (Ed.,) and Lauriault, T. (Assoc. Ed.) Developments in the Theory and Practice of Cybercartography: Applications and Indigenous Mapping, Amsterdam: Elsevier, 219-227.

Kennedy, C.C. (1970). The Upper Ottawa Valley. Pembroke, Ontario: Renfrew County Council.

Kitchin, R. and Dodge, M. (2007). Rethinking Maps. Progress in Human Geography, 31(3), 331 344.

Kitchin, R. and Tate, N.J., (Eds.) (2000). Conducting Research in Human Geography. Essex: Pearson Education Limited.

Korp, M. (1991). Earthworks: Shamanism in the Religious Experiences of Contemporary Artists in North America PhD Dissertation. Ottawa, Ontario: University of Ottawa.

Kosslyn, S. (1994). Image and Brain: The Resolution of the Imagery Debate. Cambridge: MIT Press, 405-7.

Kruzich, N. (2011). The Ancestors are Arranging Things. Ottawa: Borealis Press.

Latour, B. (1990). Drawing Things Together. Lynch, M. and Woolgar, S. (Ed.) Representation in Scientific Practice. Cambridge: MIT Press, 19-68.

Lawrence, B. (2012). Fractured Homeland: Federal Recognition and Algonquin Identity in Ontario. Vancouver University of British Columbia Press.

Le Doeff, M. (1987). Women and philosophy. In Moi, T. ed., French Feminist Thought: A Reader, Oxford: Basil Blackwell.

Legget, R. (1975). Ottawa Waterway, Gateway to a Continent. Toronto, Ontario: University of Toronto Press.

Leonard, K.C. Jr. (2003). The Beechwoods Confederacy 1709-1809. Bowie, Maryland: Heritage Books.

Leopold, A. (1949). A Sand County Almanac, and Sketches Here and There. New York: 
Oxford Univ. Press.

Levi-Strauss, C. (1962). Totemism. London: Merlin Press.

Levi-Strauss, C. (1963). Structural Anthropology. New York. Basic Books.

Levi-Strauss, C. (1966). The Savage Mind. Chicago: The University of Chicago Press.

Livingstone, D. N. (1995) The Polity of Nature: Representation, Virtue Strategy. Cultural Geographies, 2 (4), 353-377.

Lloyd, G. (1984.) The Man of Reason: 'Male' and Female' in Western Philosophy. London: Metheun. Loomba, A. (2005). Colonialsm/Postcolonialism $2^{\text {nd }}$ Edition. New York: Routledge.

Louis, R. P. (2007). Can You Hear Us Now? Voices from the Margin: Using indigenous Methodologies in Geographic Research. Geographical Research, 45(2), 130-139.

Louis, R.P., Johnson, J.T., and Pramono, A.H., (2012). Introduction: Indigenous Cartographies and Counter-Mapping. Cartographica, 47:2, 77-79.

Lovelock, J. (2009). The Vanishing Face of Gaia: A Final Warning. London; New York. Allen Lane/Penguin Books.

Malcolm, D. (2010). Speaking Plainly about Research, Governance, and Policy for Sustainable Living. Pimatisiwin: A Journal of Aboriginal and Indigenous Community Health, 8(1), 171-183.

Mallory, C. (2001). Acts of Objectification and the Repudiation of Dominance: Leopold, Ecofeminism, and the Ecological Narrative. Ethics \& the Environment, 6(2), 59-89.

Mann, C.C. (2005). 1491 New Revelations of the Americas Before Columbus. Toronto: Alfred A. Knopf.

Mann, C.C. (2011). 1493 Uncovering The New World Columbus Created. New York: Alfred A. Knopf.

Mansvelt, J., and Berg, L.D. (2010). Writing Qualitative Geographies, Constructing Meaningful Geographical Knowledges. In Hay, Ed., Qualitative Research Methods in Human Geography. Don Mills, Ontario: Oxford University Press.

Massey, D. (1993). Power-geometry and a Progressive Sense of Place. In Mapping the Futures: Local Cultures, Global Change. Bird, J. Curtis, B., Putnam, T., Robertson, G. and Tickner. L. (Eds.). London: Routledge. 59-69.

Massey, D., Allen, J., \& Sarre, P. (Eds.). (1999a). Issues and Debates. In Human Geography Today. Malden, MA: Blackwell, (3-21). 
Massey, D., Allen, J., \& Sarre, P. (Eds.). (1999b). Human Geography Today. Cambridge, UK: Polity Press.

Matsumoto, V. (1996). Reflections on Oral History: Research in a Japanese American Community. In D. L. Wolf (Ed.), Feminist Dilemmas in Fieldwork. Boulder, Colo: Westview Press, 160-169.

McCallum Ian. (2008). Ecological Intelligence: Rediscovering Ourselves in Nature. Minneapolis: Fulcrum Publishing.

McGregor, S. (2004). Since Time Immemorial: "Our Story” The Story of the Kitigan Zibi Anishinabeg. Maniwaki, Quebec: Anishinabe Printing.

McLuhan, T. C. (1972). Touch the Earth - A Self Portrait of Indian Existence. New York: Pocket Books.

Merchant, C. (1980). The Death of Nature: Women, Ecology and the Scientific Revolution. New York: Harper and Row.

Mels, T. Ed. (2004). Reanimating Spaces A Geography of Rhythms. England: Ashgate Publishing Ltd.

Mignolo, W. (2000). Local Histories/ Global Designs. Princeton: Princeton University Press.

Minteer, B., \& Manning, R. (2005). An Appraisal of the Critique of Anthropocentrism and Three Lesser Known Themes in Lynn White's “The Historical Roots of Our Ecologic Crisis”. Organization \& Environment, 18(2), 163-176.

Mitchell, P. (2008). Cartographic Strategies of Posmodernity. New York: Routledge.

Mutwa, C. V. (1964). Indaba My Children. New York: Grove Press.

Mutwa, C. (1966). The Song of the Stars. New York: Barrytown, Ltd.

Mutwa, C. (1996). Isilwani The Animal Tales and Fables of Africa. Cape Town: Struik Publishers (Pty) Ltd.

Mutwa, C. (2003). 'Forward'. In Tucker L. Mystery of the White Lions: Children of the Sun God. White River: Npvenvu Press, 9.

Murphy, P. D., (2000). Further Afield in the Study of Nature-Oriented Literature. Charlottesville. VA, and London: University Press of Virginia.

Naess, A., Rothenberg, D., (Trsl. and Ed.) (1989). Deep Ecology. Cambridge: Cambridge University Press.

McDermott, L. (2004). Exploring Intersections of Physicality and Female-only Canoeing 
Experiences. Leisure Studies, 23(3), 283-301.

Nickels, B. (2001). Native American Free Passage Rights under the 1794 Jay Treaty: Survival Under United States Statutory Law and Canadian Common Law. Boston College International and Comparative Law Review. Spring 2001, 24 (2), 313-340.

Nietzsche, F.W. 1969. On the Genealogy of Morals. Tr. Kaufmann, W. and Hollingdale, R. J. New York: Vintage.

Newbery, L. (2003). Will Any/Body Carry That Canoe? A Geography of the Body, Ability, and Gender. Canadian Journal of Environmental Education, 8, 204-216.

Ouimet, Lucie (Dir.). (2000). Ojigkwanong: Encounter with an Algonquin Sage. William

Commanda documentary film. Montreal, Quebec: National Film Board of Canada.

Palmer, C. (2001). Taming the Wild Profusion of Existing Things? A study of Foucault, power, and human/animal relationships. Environmental Ethics, 23(4), 339-358.

Paquin, J-G. (2004). Le Pays de Canard Blanc. Montpellier: Bibliotheque et Archives Canada.

Paquin, J-G. (2014). Au Pays Des Weskarinis. Cheneville: Bibliotheque et Archives Canada.

Peace, R.. and van Hoven, B. (2010). Computers, Qualitative Data, and Geographical Research. In Hay, I. (Ed.), Qualitative Research Methods in Human Geography (Third ed.,) Don Mills, ON: Oxford University Press, 295-313.

Pearce, M.W., (2009). Mapping, Non-Western. International Encyclopedia of Human Geography. Oxford: Elsevier, 372 -384.

Pearce, M. W., \& Louis, R. P. (2008). Mapping Indigenous Depth of Place. American Indian Culture Research Journal, 32(3), 107.

Perkins, C., (2008) Cartography: Mapping Theory. Progress in Human Geography, 27(3), 341351.

Perrett, R. W. (1998). Indigenous Rights and Environmental Justice. Environmental Ethics, 20(4), 377.

Pickerill, J. (2008). The Surprising Sense of Hope. Antipode, (3), 482-487.

Plummer, R. (2009). Outdoor Recreation: An Introduction. New York, NY: Routledge.

Polkinghorne, D. E. (2001). Validity Issues in Narrative Research. Qualitative Inquiry, 13(4), 471-486.

Pouyanne, V. (Dir.) (2005). Good Enough for Two!: L'art de faire un canot par William Commanda [The Art of Canoe Making by William Commanda]. William Commanda documentary film. Montreal, Quebec: Studio Loon. 
Pratt, G. (2000). Post-structuralism. The Dictionary of Human Geography. Johnston, R. J., Gregory, D., Pratt, G. and Watts, M. (Eds.). Malden: Blackwell Publishing, 625-627.

Price, J. 2008. Living Inuit Governance in Nunavut (Aupilaarjuk). In Simpson, L. Ed., Lighting the Eight Fire: The Liberation, Resurgence, and Protection of Indigenous Nations. Winnipeg, Manitoba: Arbeiter Ring Publishing, 127-138.

Pritchard, E. (1997). No Word for Time: The Way of the Algonquin People. Hong Kong: East Asian Publishing Company.

Pritchard, E. (2007). Native New Yorkers. San Francisco/Tulsa: Council Oak Books.

Pritchard, E. (2009). Henry Hudson and the Algonquins of New York. San Francisco/Tulsa: Council Oak Books.

Proctor, J. (1998). Geography, Paradox and Environmental Ethics. Progress in Human Geography, 22(2), 234-255.

Proctor, J.D. (1999). Introduction: Overlapping Terrains. In J.D. Proctor \& D.M. Smith (Eds.), Geography and Ethics: Journeys in a Moral Terrain New York, NY: Routledge, 1-16.

Proctor, J.D. (2009). Environment After Nature: Time for a New Vision. In J.D. Proctor (Ed.), Envisioning Nature, Science, and Religion. West Coushohocken, PA: Templeton Foundation Press, 1-14.

Pyne, S. and Taylor, F. D. R. (2012). Mapping Indigenous Perspectives in the Making of the Cybercartographic Atlas of the Lake Huron Treaty Relationship Process. Cartographica, Special Issue on Indigenous Cartography and Counter Mapping, 47 (2), 92-104.

Quest for Fire Movie. (1981). Director: Annaud, J-J.

Raffan, J. Ed. (2005). Rendeqvous with the Wild: The Boreal Forest. Erin, Ontario: Boston Mills Press.

Rajnovich, G. (1994). Reading Rock Art. Toronto: Natural Heritage/Natural History Inc.

Robinson, Arthur. (1952). In the Look of Maps. Madison: University of Wisconsin Press.

Rolston, H. and Light. A. (2002). Environmental Ethics. Theological Studies, 66(4), 783-814.

Rose, G. (2000). Practicing Photography: An Archive, a Study, Some Photographs and a Researcher. Journal of Historical Geography 26(4), 555 -571.

Rose, G. (2017). Posthuman Agency I the Digitally Mediated City: Exteriorization, Individuation, Reinvention. Annals of the American Association of Geographers. 107(4), 779-793.

Routley, R. (1973). Is There a Need For a New, an Environmental, Ethic? XVth World Congress of Philosophy, Sofia, Bulgaria. 
Roszak, T. (1973). Where the Wasteland Ends. New York: Anchor Books.

Rundstrom, R. A. (1991) The Changing Direction of North American Cartography. Cartographica, 28(2), 1-12.

Rundstrom, R., (2009). Counter-Mapping. International Encyclopedia of Human Geography. Oxford: Elsevier 1-5.

Said, E. W. (1979). Orientalism. New York, USA: Random House Inc.

Schaefer, J. (2005). Valuing Earth Intrinsically and Instrumentally: A Theological Framework for Environmental Ethics. Theological Studies, 66(4), 783-814.

Schoolcraft, H. R. (1851-1857). Historical and Statistical Information Respecting the History, Condition, and Prospects of the Indian Tribes of the United States Six Volumens. Philadelphia: Lippincott, Gambo, and Co.

Shaw, Wendy S., Herman, R.D.K., Dobbs, G. Rebecca. (2006). Encountering Indigeneity: Re-imagining and decolonizing geography. Swedish Society for Anthropology and Geography, 267276.

Shields, R. (1991). Places on the Margins: Alternative Geographies of Modernity. New York, NY: Routledge.

Shiva, V. (1997). Biopiracy: The Plunder of Nature and Knowledge. Boston, MA: South End Press.

Shiva, V. (2005). Earth Democracy: Justice, Sustainability, and Peace. Cambridge, Mass: South End Press.

Simpson, L. Ed. (2008). Lighting the Eight Fire: The Liberation, Resurgence, and Protection of Indigenous Nations. Winnipeg, Manitoba: Arbeiter Ring Publishing.

Sioui, G. (2008). Histoires de Kanatha Vues et Contees, Histories of Kanatha Seen and Told. Ottawa, Ontario: University of Ottawa Press.

Smith, E. L. (2007). River Restoration Through Dam Removal Efforts, with a Particular Focus on the Ottawa River at Chaudière Island Discussion Paper. Asinabka. http://www.asinabka.com/Archives/Chaudiere_restoration_11july07.pdf.

Smith, E. L. (2011). An Urban Epicentre of Decolonization in Canada: the Indigenous-Settler Alliance to Make a Place for Peace at Asinabka. Thesis http://www.asinabka.com

Smith, M. (2001). The Face of Nature: Environmental Ethics and the Boundaries of Contemporary Social Theory. Current Sociology, 49(1), 49-65.

Smith, S. (1993). Who's Talking/Who's Talking Back? The Subject of Personal Narrative. Signs: Journal of Women in Culture and Society, 18(2), 408 - 425. 
Smith, L. T. (2012). Decolonizing Methodologies: Research and Indigenous Peoples. London, New York: Zed Books.

Smith, D. W. (2013). Sanford Encyclopaedia of Philosophy On-Line Discussion of Phenomenology (dwsmith@uci.edu).

Some, M. P. (1994). Of W ater and the Spirit. New York: The Penguin Group.

Some, M. P. (1999). The Healing Wisdom of Africa. London: Thorsons.

Soja, E. (1989). Postmodern Geographies: The Reassertion of Space in Critical Social Theory. London: Verso Press.

Soja, E. (1996). Thirdspace: Journeys to Los Angeles and Other Real-and-Imagined Places. Oxford: Basil Blackwell.

Spivak, G. C. (1988). Can the Subaltern Speak? In, Marxism and the Interpretation of Culture. Ed. Nelson, C., and Grossberg, L. Urbana: University of Illonois Press. 271-313.

Spivak, G. C., (1999). A Critique of Postcolonial Reason. Cambridge, MA: Havard University Press.

Springer, S. (2017). Earth Writing. GeoHumanities. 3(1), 1-19.

Stokes, P. (2008). Philosophy 100 Essential Thinkers. London: Artucus Publishing Ltd.

Strivers, C. (1993). Reflections on the Role of Personal Narrative in Social Science. Signs: Journal of Women in Culture and Society 1993, 18(2), $392-407$.

Sullivan, W. (1996). The Secret of the Incas. New York: Crown Publishers Inc.

SunBow (2013) Video Tribute to the Legacy of a Legendry Peacekeeper. Quebec: Iron Wood Log Project https://youtu.be/Psax2u_rDO4

Swazo, N. K. (2005). Research Integrity and Rights of Indigenous Peoples: Appropriating Foucault's Critique of Knowledge/Power. Studies in History and Philosophy of Biological and Biomedical Sciences, 36568.

Taylor, D. R. F. (1997). Cybercartography: Theory and Practice, Volume 4. In Modern Cartography Series, Amsterdam: Elsevier, 574.

Taylor, D. F. R. (2003). The Concept of Cybercartography. In M. P. Peterson (Ed) Maps and the Internet. Amsterdam: Elsevier, 405-420.

Taylor, D. R. F. (2005). Cybercartography: Theory and Practice, Volume 4 in Modern Cartography Series, Amsterdam: Elsevier, 574.

Taylor, D. F. R. (2009). Maps, Mapping and Society: Some New Directions. In Proceedings of 
Global Map Forum, Tskuba, Japan: Geographical Survey Institute, 32-35.

Taylor, D. F. R. and Pyne, S. (2010). The History and Development of the Theory and Practice of Cybercartography. International Journal of Digital Earth, 3(1), 1-14.

Taylor, D. F. R., Ed., and Lauriault, T. Assoc. Ed. (2014). Developments in the Theory and Practice of Cybercartography: Applications and Indigenous Mapping, Amsterdam: Elsevier

Tafoya, T. (1995). Finding Harmony: Balancing Traditional Values with Western Science in Therapy. Canadian Journal of Native Education, 7-27.

Thomas, R. A. (2005). Honouring the Oral Traditions of My Ancestors Through Storytelling. In L. A. Brown, \& S. Strega (Eds.), Research as Resistance: Critical, Indigenous and Anti-oppressive Approaches. Toronto: Canadian Scholars' Press.

Thoreau, H. D. (1906). Natural History in the Writings of Henry David Thoreau. Boston: Houghton Mifflin.

Thoreau, H. D. (1964). The Portable Thoreau Walden Pond, Essays, Poems, Journal. Bode, C. (Ed.) New York: The Viking Press.

Thumbadoo, R.V. (2005). Learning From a Kindergarten Dropout. Ottawa: Ottawa, Ontario: Circle of All Nations.

Thumbadoo, R.V. (2008). Learning from a Kindergarten Dropout Book Two. Ottawa, Ontario: Circle of All Nations.

Thumbadoo, R.V. (2008). Passionate Waters- Butterfly Kisses. Ottawa, Ontario: Circle of All Nations.

Thumbadoo, R.V. (2016). Audio interview with Prophecy-keeperz https://m.soundcloud.com/mackboogaloo/prophecy-keeperz-romola-thumbadoo-ep60the-legacy-of-william-commanda

Thumbadoo, R.V. (2015). Video of Romola V. Thumbadoo's presentation on the Asinabka Vision (Asinabka Sacred Chaudiere Site Timeline Development History) at the 2014 Pbil Ochs Festival, Ottawa, Ontario chevron-down

Thwaites, R. G. Ed. (1896-1901). The Jesuit Records and Allied Documents. Seventy-Three Volumes. Cleveland: The Burrows Brothers Co.

Tolman, E.C. (1948). Cognitive Maps in Rats and Men. Psychological Review, 55, 189 -208.

Tucker L. (2003). Mystery of the White Lions: Children of the Sun God. White River: Npvenvu Press.

Turnbull, D. (2007). Maps Narratives and Trails: Performativity, Hodology and Distributed 
Knowledges in Complex Adaptive Systems - an Approach to Emergent Mapping. Geographical Research. June 2007. 45(2) 140-149.

Timreck, T. (2010). Great Falls: Discovery, Destruction and Preservation in a Massachusetts Town Video Documentary. Great Falls, Massachusetts: www.hiddenlandscape.com.

Valentine, G. (2003). Geography and Ethics: in Pursuit of SocialJ; Ethics and Emotions in Geographies of Health and Disability Research. Progress in Human Geography. 27 (3), 375-80.

Valentine, G. (2004). Geography and Ethics: Questions of Considerability and Activism in Environmental Ethics. Progress in Human Geography, 28(2), 258-263.

Vastokas, J. M. and R. K. (1973). Sacred Art of the Algonkians: A Study of the Peterborough Petroglyphs. Peterborough, Ontario: Mansard Press.

Vidal de La Blache, P. (2010). In Encyclopædia Britannica. Retrieved October 20, 2010, from Encyclopædia Britannica Online: http://www.britannica.com/EBchecked/topic/627886/Paul-Vidal-de-la-Blache

Watts, M. (2000a). Colonialism. The Dictionary of Human Geography. $4^{\text {th }}$ Ed. Johnston, R. J., Gregory, D., Pratt, G., and Watts, M. (Eds.). Malden: Blackwell Publishing, 93-95.

Watts, M. (2000b) Political Ecology. The Dictionary of Human Geography. $4^{\text {th }}$ Ed. Johnston, R. J., Gregory, D., Pratt, G., and Watts, M. (Eds.). Malden: Blackwell Publishing, 590-592.

Watts, M. (2001) The Holy Grail: In Pursuit of the Dissertation Proposal. Institute of International Studies, 1-12.

Waitt, G. (2010). Doing Foucauldian Discourse Analysis - Revealing Social Identities. In Hay, I. (Ed.), Qualitative Research Methods in Human Geography (3th ed.,) Don Mills, Ontario: Oxford University Press.

Whatmore, S., \& Thorne, L. (1998). Wild(er)ness: Reconfiguring the Geographies of Wildlife.Transactions of the Institute of British Geographers, 23(4), 435-454.

White, L. Jr. (1967). The Historical Roots of our Ecologic Crisis. Science, 155, 1203-1207.

Wildcat, D. (2001). The Schizophrenic Nature of Western Metaphysics. Power and Place:

Indian Education in America. Vine Deloria, Jr. and D. Wildcat (Eds.) Golden, Colorado:

Fulcrum, 47-55.

Wilson, Alexander. (1991). The Culture of Nature: North American Landscape from Disney to the Exxon Valdez: Toronto: Between the Lines Press. 
Wilson, S. (2008). Research Is Ceremony: Indigenous Research Methods. Black Point: Fernwood Publishing.

Wise, J. C. (1971). Deloria, Vine, Jr. (Ed.) The Red Man and the New World Drama. New York: The Macmillan Company.

Woodward K., Dixon, D.P., Jones, J.P.III. (2009). Poststructuralism/Poststructuralist Geographies, 396-407.

Woodward, D. and Lewis, G. M. (Eds.) (1998). The History of Cartography Volume Two, Book Three - Cartography in the Traditional African, American, Arctic, Australia, and Pacific Societies. Chicago: University of Chicago Press.

Wood, J. (2005). How Green is My Valley? Desktop Geographic Information Systems as a Community-based Participatory Mapping Tool. Royal Geographical Society (with The Institute of British Geographers), 37(2), 159-170.

Wright Carr-Harris, B. (1903). The White Chief of the Ottawa. Toronto: William Briggs.

Wright, R. (2009). What is America? Toronto: Vintage Canada Edition.

Wright, R. (2004). A Short History of Progress: CBC Massey Lectures. Toronto: House of Anansi Press Inc. 


\section{A Special Addendum to the Thesis Bibliography}

\section{William Commanda's Home Library - A partial compilation}

Arden, H. and Wall, S. (1990). Wisdom Keepers. Hillsboro, Oregon: Beyond Words Publishing Inc.

Asch, M. (ed.) (1997). Aboriginal and Treaty Rights in Canada: Essays on Law, Equality and Respect for Difference, Vancouver: UBC Press.

Bideaux, M. (1986). Relations-Jacques Cartier. Montréal: Les presses de l'université de Montréal.

Blomley, N. (1994). Law, Space and the Geographies of Power. New York: Guilford Press.

Bon, F.L. (1994). New England Indians: Biographies and Legends, Vol III. Burlington, Maine: Pride Publication Inc.

Bon, F.L. (1970). New England Indians: Biographies and Legends, Vol II. Burlington, Maine: Pride Publication Inc.

Bowen, E. (ed.) (1973). The Indians of Canada Text by Capps Benjamin. Alexandria, Virginia: Time Life Books Inc.

Burland, C. (1970). The People of the Ancient Americas. Toronto: Himalayan Publishing Group.

Burns, B. (1993). Sitting Bull and other Legendary Native American Chiefs. New Jersey: Random House Company.

Buttree, J. M. (1930) The Rhythm of the Red Man. United States: A. S. Barnes and Company. Central Cree and Ojibway Crafts Household Accessories: Bark. Ottawa: Indian and Northern Affairs

Calihoo, R. and Hunter, R. (1991). Occupied Canada. Toronto: McClelland and Stewart Inc.

Callwood, J. (1981). Portrait of Canada. New York: Double Day and Company Inc.

Cardinal, H. (1999). Unjust Society. Vancouver: Douglas and McIntyre Ltd.

Carter, S. (1990). Lost Harvests, Prairie Indian Reserve Farmers and Government Policy. Montreal: McGill-Queens University Press.

Colden, C. (1972). The History of the Five Indian Nations of Canada. Toronto: Coles Publishing Company.

Constable, G. (ed.) (1975). The Great Chiefs Text by Capps Benjamin. Alexandria Virginia: Time Life Books Inc.

Crump, J. (2003). The War of 1812 Against the States. Canmore, Ont: .Altitude Publishing. 
Davidson, R. (1993). Eagle of the Dawn. Vancouver: Douglas and McIntyre.

Davis, C. (1969). North American Indian. Toronto: The Hamlyn Publishing Group.

Dempsey, H. (1982). History in their Blood. Vancouver: Douglas and McIntyre Ltd.

Dickie D.J., Palk, H. and Woodley E.C. (1952). My Country's Story. Toronto: J. M. Dent and Sons.

Dumas, L. (1992). The Amerindians and Inuit in Today's Quebec. Quebec: Les publications du Quebec.

Eckert, A. W. (1993). A Sorrow in our Hearts. The Life of Tecumseh. New York: Bantam Books.

Frideres, J.S. (1983). Native People in Canada Contemporary Conflicts. Scarborough, Prentice Hall Canada Inc.

Garnier, K. (1990). Our Elders Speak. Burnaby, B.C.: Hemlock Printers Ltd.

Geggie, N. (2003). Wakefield Revisited. Chelsea, Quebec: Castenchel Editions.

Geographic Board of Canada (1971). Handbook of Indians of Canada. Toronto: Coles

Publishing Company.

Grant, B. (1961) Leather Braiding. Cambridge: Cornell Maritime Press Inc.

Harriot, T. (1972) A Brief and True Report of the New Found Land of Virginia. New York: Dover Publication.

Hawkins, M. (1983). Here to Stay. Ottawa: MOM Printing.

Hedgpeth, D. and Walt, R. (1993). The Art of Tom Lovell: An Invitation to History. New York: William Morrow and Company.

Helly, M. and Courgeon, R. (1996). Monteruma and the Aztecs. New York: Henry Holt and Company Inc.

Howley, J.P. (1980). The Beothucks or Red Indians. Toronto: House of Anansi.

Jenness, D. (1932). The Indians of Canada. Toronto: University of Toronto Press.

Johnson, S. (1994). The Book of Elders. New York: Harper Collins Publishers.

King, T. (2003). The Truth about Stories: A Native Narrative. Toronto: House of Anansi Press Inc.

Krotz, L. (1980). Urban Indians. Edmonton, Hurtig Publishers Ltd.

Johnston, B.H. (1988). Indian SchoolDays. Toronto: Key Porter Books Ltd. 
La Farge, O. (1956). A Pictorial History of the American Indian. New York: Crown Publishers.

La Farge, O. (1956). The American Indian. New York : Crown Publishers Inc.

Larouche, P. (1992). Montréal 1535 La redécouverte de Hochelaga. Outremont. Les éditions Villes Nouvelles-Villes.

Leach, G.P. and Humbert, G. J. (1989). Beedabbun-First Light of Dawn Anishinabe Spiritual Center, Espanola Ont. North Bay, Ontario: Tomiko Publications.

Lepage, P. (2009). Aboriginal Peoples Fact and Fiction. Quebec: Imprimerie Provinciale Inc.

McMaster, G. and Martin, Lee-Ann (ed.) (1992) Indigena Contemporary Native Perspectives.

Vancouver: Douglas and McIntyre Ltd.

Mails, T.E. and Evehema, D. (1995). Hotevilla: Hopi Shrine of the Covenant Microcosm of the World. New York: Marlow and Company.

Marist Brothers. (1923). Canada and the Province of Quebec. Granger Frères, Ltd.

Martyn, J.W. (1993). Peterborough Doctors: The Past is Simply a Beginning. Ontario: Gymel.

Mason, J. (ed.) 1974. The Sportsman Wilderness. New Jersey: Pound Books.

Masta, H.L. (1932). Abenaki Indian Legends Grammar and Place Names. Victoriaville:

L'imorimrire de Victoriaville.

Maxwell, J. A. (ed.) (1978). America's Fascinating Indian Heritage. New York: Reader's Digest Inc.

Moore, R.J. Jr. (1997). Native American, a Portrait. Don Mills, Ontario: General Publishing Company Ltd.

Nagelfell, K.(ed.) (1995). North American Indian Chiefs. Maine: JG Press Inc.

Paper, J. (1998). Offering Smoke Idaho: University of Idaho Press.

Pelletier, W. and Poole, T. (1973). No Foreign Land. New York: Random House.

Point, S.A. (2000). Coast Salish Artist. Vancouver: Douglas and McIntyre Ltd.

Pritchard, E. (1997). No Word for Time: The W ay of the Algonquin People. Hong Kong: East Asian Publishing Company.

Remini, R.V. (2001). Andrew Jackson and his Indian Wars. New York: Penguin Putman Inc. Reynolds, A. and Wright, L. (2000). Cycles: A View of Planet Earth. South A frica: Pondsview 
Publishing.

Rutledge, J.L. (1956) Century of Conflict. New New York: Double Day and Company Inc.

Sams, J. and Nitsch, T. (1991). Other Council Fires Were Here Before Ours. New York: Harper Collins Publishers.

Shell Canada Ltd. (1987) The Spirit Signs: Artistic Traditions of Canada's First People. Toronto: McClelland and Stewart Inc.

Stoutenburgh, J.L. Jr. (1960). Dictionary of the American Indian. New York: Random House Company.

Sugden, J. (1997). Tecumseh: A Life. New York: Henry Holt and Company Inc.

Sugden, J. (2000). Blue Jacket W arrior of the Shawnees. Nebraska: University of Nebraska Press.

Swanson, W. (2004). Why the West was Wild. Richmond Hill: Fire Fly Books.

Viola, H. J. (1990). After Columbus. New York: Crown Publishers Inc.

Walker, S.L. (1993). The South West. Bellmont Arizona: Canyonlands Publications.

Warner, J.A. (1975). The Life and Art of the North American Indian. Toronto: Himalayan Publishing Group.

Wells, H. G. (1956). The Outline of History I. New York: Garden City Books.

White, A. T. (1961). The American Indian. New York: American Heritage Publishing.

Wise, J. C. (1971). Ed. Deloria, Vine, Jr. The Red Man and the New World Drama. New York: The Macmillan Company.

2. William Commanda also compiled a large video/documentary collection. This is yet to be catalogued.

\section{The Following Books and Videos Document William Commanda's Work}

The Indian Crafts of William and Mary Commanda 1967 David Gidmark

Grandfather William Commanda A Few Reflections on the Very Special Occasion of your Ninetieth Birthday 2003 Circle of All Nations Colleagues

Learning from a Kindergarten Dropout 2005 R.V. Thumbadoo

Learning from a Kindergarten Dropout Book Two 2008 R.V. Thumbadoo

Passionate Waters Butterfly Kisses Kayak Photo Journal 2008 R.V. Thumbadoo

William Commanda Biography in Up the Gatineau Vol 40 historical publication, 2014

Ojijkwanong Encounter with an Algonquin Sage, Lucie Ouimet 2002 NFB documentary 
Circle of All Nations Gathering 2001 documentary

Good Enough for Two canoe making documentary, 2003 Valerie Pouyanne, Loon Productions The Awakening, 2007 Jeremy Wright

Mishomis William Commanda, 2008 Ironwood Log Project SunBow

Portage of Wisdom, 2009 Patrick Gravel

Inner Healing Indigenous Trees of Wisdom, 2014 Adrien Esposito documentry

In addition, since 2009, Circle of All Nations has also been a presence in social media via several facebook pages:

William Commanda

William Commanda Legacy Asinabka

Circle of All Nations

Romola's Photo Page Nature CAN TEACH

4. Sunbow, Ironwood Log, A Circle of All Nations Colleague compiled the following two lists of on-line references to Grandfather William Commanda after his death in 2011. Not all the links may be active, since they were created and shared by his widespread community friends in the cyberworld.

William Commanda films, videos, audio:

1976 Festival of American Folklife, Smithsonian:

http:/ / aifg.arizona.edu/film/1976-festival-american-folklife-5-algonkin

Cry of the Earth, UN, 1993:

http://www.youtube.com/watch?v $=$ MxdR4ZyOWPA\&feature $=$ youtu.be

CBC and other videos:

http://storify.com/cbcnews/william-commanda-19132011

l'UQO rend hommage à William Commanda:

http://www.youtube.com/watch?v $=$ qUhq8ToW7HM

William Commanda et la Prophétie des 7 Feux:

http://jean-louismaillard.skyrock.com/3145886230-William-Commanda-et-la-prophetie-des-7-feux.html

L'Appel du 7ème Feu:

http://www.medecinewakan.com/vid \%C3\%A9os.php

http://www.authorstream.com/Presentation/Mariediane-1482290-appel-du-7e-feu-selon-la-proph-tiewilliam-commandades-8-feux/

Dr Commanda Willis Scholarship 2005:

http://www.wansfilm.net/movies/dr-william-commanda-willis-scholarship.html

Mishomis William Commanda, Ironwood Log Project:

Presented at A Circle of All Nations in 2008:

http://www.isuma.tv/fr/ironwood-log-project/william-commanda

Asinabka online 2010:

http:/ / www.youtube.com/watch?v $=u F C 6 a S S m R s 0 \& f e a t u r e=y o u t u . b e$

NAAA Award 2010:

http://www.oqiton.com/william-commanda-video/

http://tv.powwows.com/video/2012/08/16/william-commanda-au-naaa-2010-musique-biographique-louis-amik/\#

Ojigkwanong - Encounter with an Algonquin Sage, NFB 2010: 
http:/ $/$ www3.nfb.ca/enclasse/doclens/visau/index.php? mode $=$ theme\&language $=$ english $\&$ theme $=30662 \&$ film $=50242 \&$ ex cerpt $=612175$

Great River Documentary, clip 2011:

http://www.youtube.com/watch?v=uGGdcr97Sdk\&feature=youtu.be http://geegeesgraffiti.blogspot.ca/2011/02/william-commanda-algonquin-elder.html http://lemaymedia.blogspot.ca/2010/04/valley-variety-william-commanda.html

Ojigkwanong - Rencontre avec un sage algonquin, ONF, 2010:

http:/ $/$ www3.onf.ca/enclasse $/$ doclens $/$ visau/index.php?excerpt $=612174 \&$ film $=50087 \&$ language $=$ french\&mode $=$ theme $\&$ theme $=30662$

Song performed by William Commanda:

http://www.museevirtuel-virtualmuseum.ca/sgc-cms/histoires_de_chez_nous-

community_memories/pm_v2.php?id=record_detail $\& \mathrm{fl}=0 \& \mathrm{lg}=$ Francais $\&$ ex $=00000369 \& \mathrm{rd}=156030$

Hommage à William Commanda 2011:

http://99mp3.org/play.php?id=qUhq8ToW7HM\&t=LUQO $\% 20$ rend $\% 20$ hommage $\% 20 \% \mathrm{C} 3 \%$ A0 $\% 20$ William $\% 20$ Comm $\underline{\text { anda }}$

http://99mp3.org/mp3-download-l-uqo-rend-hommage- $\% \mathrm{C} 3 \% \mathrm{~A} 0$-william-commanda.html

Portage des sagesses, 2011:

http://samuelallo.com/index.php?option=com_content\&view=article\&id=17\&Itemid=33

Portage of Wisdom, 2011:

http://ottawa.bibliocommons.com/item/show/709260026_william_commanda

William Commanda: Tribute to the legacy of a legendary Peacemaker.

Ironwood Log Project, released on Grandfather's 100th birthday,

https://www.youtube.com/watch?v=Psax2u_rDO4

\section{William Commanda Bibliography}

Image search:

https: $/ /$ www.google.ca/ search?q $=$ william + commanda\&newwindow $=1 \&$ safe $={ }_{\text {off }} \&$ client $=$ firefox-

a\&hs $=$ Ho\&rls $=$ org. mozilla:fr:official\&tbm $=$ isch\&tbo $=$ u\&source $=$ univ\&sa $=$ X\&ei $=$ hjJ4UtrQAsjhyQHE44Ew\&ved $=0$ CC8 QsAQ\&biw $=1024 \&$ bih $=579$

In memoriam, Flicker photos:

http://www.flickr.com/photos/gazetteuogazette/6006233776/in/photostream/

Photos 1980:

http://www.oqiton.com/my-teacher/

Photo:

http://www.erictemple.com/keyword/commanda\#!li=1417137580\&k=rn56CGD

Portrait:

http://www.zhibit.org/nicolemcgrath/studio-work-2008-2011/portrait-of-algonquin-elder-william-commanda

Out'Art, portrait:

http://www.outart.ca/francine_dugas.html

Wikimedia Commons:

http://commons.wikimedia.org/wiki/File:William_Commanda.jpg

Photobucket:

http://photobucket.com/images/Grandfather+William+Commanda,+ALGONQUIN/?page=1

Wikipedia:

http://en.wikipedia.org/wiki/William_Commanda 
Native American Encyclopedia:

http://nativeamericanencyclopedia.com/william-commanda/

Native leaders of Canada:

http://www.newfederation.org/Native_Leaders/Bios/Commanda.htm

Algonquin Chiefs of Kitigan Zibi:

http://kzadmin.com/Chiefs.aspx

Academic dictionaries:

http://en.academic.ru/dic.nsf/enwiki/6511113

William Commanda Legacy

https://www.facebook.com/pages/William-Commanda-Legacy/701369829887220? fref $=$ ts

Facebook, William Commanda:

https://www.facebook.com/pages/William-Commanda/124359520927182

Facebook, Circle of All Nations:

https://www.facebook.com/pages/Circle-of-All-Nations/127700713932537

A Circle of All Nations:

http://www.circleofallnations.ca/

http://www.angelfire.com/ns/circleofallnations/page2.html

William Commanda's blog:

http://circleofallnations.blogspot.ca/2006/02/photo-gallery.html

A mini-biography (pdf):

http://www.renaud.ca/public/Letters_of_Support/2011-01-05_GWC_MiniBio.pdf

Chronology of key activities, angel fire:

http://www.angelfire.com/ns/circleofallnations/V1.html

Conversation of Four Elements, Wisdom of the First Nations, Treaty Room, Washington DC 1994:

http://www.ratical.org/ratville/future/william.html

Letter to William Commanda about the wampum belts, 1994:

http://claudeaubinmetis.com/viewtopic.php?f $=18 \& \mathrm{t}=166$

Sunbow 5 Walk, Cry of the Earth, UN, Odyssey of the $8^{\text {th }}$ Fire 1995:

http://www.8thfire.net/Day_15.html

Sunbow 5 Walk 1995:

https://groups.google.com/forum/\#!topic/soc.culture.asian.american/8o79N5DF_kg

Sunbow 5 Walk:

http://www.hartford-hwp.com/archives/41/381.html

Elders without borders:

http://www.elderswithoutborders.org/main/

Grandmother Pa'Ris'Ha:

http://loaradionetwork.com/grandmother-PaRisHa.html

Harmony Award 2000:

http://www.harmony.ca/awards/harmony-award/2000-elder-william-commanda/

Praise a Worthy Man: Grandfather Commanda at age 90 (2003):

http://chiron-communications.com/chirons-cave/essays-observations-profiles/let-us-now-praise-a-worthy-man-

grandfather-william-commanda-at-age-90/

Queen's University visitorship 2004 (pdf):

http://www.queensu.ca/studentaffairs/fundsandawards/sutherland/DistinguishedVisitors.pdf 
Wolf Award 2005:

http://www.wolfproject.com/organizations.htm

University of Ottawa, Honorary Doctorates 2005:

http://www.president.uottawa.ca/doctorate-details_716.html

Aboriginal Affairs, Government of Canada, Dr. William Commanda Scholarship:

https://www.aadnc-aandc.gc.ca/eng/4078514441652/4078514441652

Dr William Commanda scholarship, Willis College (pdf):

http://www.williscollege.com/pdf/w_commanda_scholarship_application.pdf

Dr. William Commanda Willis Scholarship:

http://www.teknowave.ca/wcommanda/index.htm

Key to the City of Ottawa 2006 (pdf):

http://www.destinybydesign.ca/CANposters/2006GWCMidYr1to7.pdf

Address at the Public hearings regarding the Mega-Dump project, June 2007:

http://www.bape.gouv.qc.ca/sections/mandats/LET-danford-lake/documents/DM68.pdf

Grandfather turns 94, 2007, Odawa Native Friendship Center (pdf):

http://www.odawa.on.ca/posters/William_Commanda_94th_birthday.pdf

Manataka, Message from Grandfather 2008:

http://www.manataka.org/page1458.html

Democratic underground, Equinox 2008, Message from Grandfather:

http://www.democraticunderground.com/discuss/duboard.php?az=view_all\&address $=245 \times 76578$

Letter from Grandfather William to the $8^{\text {th }}$ Fire gathering 2008 (pdf):

http://www.sacredearthnetwork.org/ns-gathering/documents/WilliamCommandablessing.pdf

Event 2009, Odawa Native Friendship Center:

http:// forum.autochtones.ca/viewtopic.php?f=2\&t=4216

Turtle Island Native Network, Order of Canada 2009:

http:/ / www.turtleisland.org/discussion/viewtopic.php? $\mathrm{f}=22 \& \mathrm{t}=6668$

Metro, Commanda awarded the Order of Canada 2009:

http://metronews.ca/news/ottawa/91784/commanda-awarded-the-order-of-canada/

\section{National Aboriginal Acbievement Foundation 2010}

http://archive.is/lbAkd

NAAA Lifetime Achievement Award 2010 (pdf):

http://www.dannybeaton.ca/uploads/NAAA_Lifetime_Achievement_Award_Grandfather_William_Commanda.pdf

The vision for Asinabka 2010:

http://ottawa.ca/calendar/ottawa/citycouncil/occ/2010/11-19/cpsc/02-

$\% 20$ report $\% 20$ on $\% 20$ the $\% 20$ vision $\% 20$ for $\% 20$ the $\% 20$ Askiabka.htm

Algonquin words ignored, A Sacred Forest must be saved,

Letter from Grandfather to the Premier of Ontario, January 2011:

http://www.ottawasgreatforest.com/Site/Algonquin_Information.html

Prayer Vigil for the Earth, Seven Fires Prophecy:

http://www.oneprayer.org/Seven_Fires_Prophecy.html

Turtle Lodge, Learning from a Kindergarten Dropout:

http://www.turtlelodgetradingpost.ca/products/index.php?route=product/product\&product_id=109

Learning from a Kindergarten Dropout:

http://www.fncaringsociety.com/fr/publications/1282 
The awakening:

http://www.thealgonquinway.ca/French/resources-detail-f.php?book=123

Sacred Belt, A Vision for Canada, Canada Day 2012:

http://browse.feedreader.com/c/No_Sound_Mind/20540486

Interview with Annie Smith St-George:

http://montrealserai.com/2013/06/23/8392/

Miskojonia, Red Gold Feat, William Commanda:

http://www.amazon.com/Miskojonia-Gold-Feat-William-Commanda/dp/B005MVJCV0

Historical canoe builder:

http://www.wcbguild.com/history.html

Canadian Canoe Museum:

http://paddle.canoemuseum.ca/index.php/adopt-an-artifact/17-adopt-a-canoe/173-commanda-birch-bark-canoe-2001-2-1

Commanda and canoe man Kirk Wipper:

http://www.kirkwipper.ca/william-commanda-and-kirk-wipp/

Evan Pritchard with Chief Commanda:

http://ravenredbone.wordpress.com/tag/chief-william-commanda/

William Commanda, link, bark canoe:

http://www.oqiton.com/william-commanda-links/

William and Mary canoe:

http://www.janicetantonblog.com/in-the-studio/undercurrents-the-red-canoe-series/attachment/commandadetail

Ottawa Citizen, Commanda canoe restored 2013:

http://www.ottawacitizen.com/travel/Commanda+canoe+being+restored+life/8808353/story.html

The Indian crafts of William and Mary, David Gidmark:

http://www.amazon.ca/Indian-crafts-William-Mary-Commanda/dp/0811725499

http://books.google.ca/books/about/The_Indian_crafts_of_William_and_Mary_Co.html?id=k0uxAAAAIAAJ\&redir_esc 三y

http://www.barnesandnoble.com/w/the-indian-crafts-of-william-and-mary-commanda-davidgidmark/1000107395?ean=9780811725491

http://www.abebooks.fr/Indian-Crafts-William-Mary-Commanda-David/5091716991/bd

David Gidmark, learning to make a birchbark canoe:

http://books.google.ca/books?id=B5gMBu0TZXEC\&pg=PA206\&lpg=PA206\&dq=william + commanda\&source=bl\&ots $=\mathrm{Q} 0 \mathrm{U}-$

_p2PEy\&sig=Hy1mRO7OUn2AIK2NIPVhcLKg5iU\&hl=fr\&sa=X\&ei=Y514UqOGJeXo2AWUt4DICA\&ved=0CD4Q6

AEwAjigAQ\# $\#_{\mathrm{v}}=$ onepage $\& \mathrm{q}=$ william $\% 20$ commanda\& $\mathrm{f}=$ false

Paddle for Peace 2010 (pdf):

http://www.birchbarkcanoes.ca/media/paddle-for-peace.pdf

Paddle for Peace 2011, Greener Springs:

http://greenersprings.webs.com/forestshumanrights.htm

Paddle for Peace, Victoria Island 2013 (pdf):

http://www.spirit-wrestlers.com/Ottawa_Peace_Festival/2013/2013-Sept-21_Paddle-for-Peace.pdf

Grandfather Commanda's legacy, Paddle for Peace 2013:

http://allevents.in/la\%20victoria/grandfather-commandas-legacy-paddle-for-peace/639044586127467

Canoe trip to DC for water protection, October 2012:

http://www.centretownnews.ca/index.php?option=com_content\&task=view\&id=3364\&Itemid=94

Heritage canoe, May 2013: 
http://www.recorder.ca/2013/05/31/heritage-canoe-to-greet-tall-ships

Tribute canoe project:

http://djpaulcorby.blogspot.ca/2011/08/tribute-canoe-project-for-grandfather.html

Canadian Heritage, Government of Canada:

http://www.pch.gc.ca/eng/1379684534336/1379948913237

Douglas Cardinal, Architect:

http://www.djcarchitect.com/04press_articles.html

Spiritual gathering in Maniwaki:

http://www.earthdrum.com/a_spiritual_gathering.htm

Learning from Mother Earth:

http://www.thefreelibrary.com/Still+learning+from+Mother+Earth $\% 3 \mathrm{~A}+$ William+Commanda, + an + aboriginal...a0126583064

Experience project:

http://www.experienceproject.com/stories/Want-You-To-Know-About-Elders-Meditation/869484

Asinabka, William Commanda's passing (pdf):

http://asinabka.com/Archives/2011_William_Commanda_Passing.pdf

Elder Commanda, chronology:

http://www.flutrackers.com/forum/showthread.php?t=7154

Commanda's tipi on canvas:

http://karstaddailypaintings.blogspot.ca/2011/08/commandas-teepee-oil-on-canvas-5-x-7-in.html

The legacy of William Commanda, Ottawa Library 2012:

http://ottawastart.com/story/17885.php

Ottawa Peace Festival, photo exhibit on Grandfather, 2012:

http://mccottawa.ca/stories/news/ottawa-peace-festival

Ottawa Peace Festival, 2013:

http://ottawapeacefestival.blogspot.ca/2013/08/ottawa-peace-festival-2013.html

Commanda's legacy, SNAP:

http://snapqc.org/blog/william-commandas-legacy/

Sixth Annual Ottawa Peace Festival to honour visionaries:

http://www.ceasefire.ca/?p=12764

Native strength:

http://nativestrength.com/tag/william-commanda/

NCC leaders urge the federal government to rename a city landmark after W, Commanda:

http://www2.canada.com/ottawacitizen/news/city/story.html?id=f99d9c81-b6d1-4719-99b3-d2cd604dc3d1

Remembering William Commanda on National Aboriginal Day

http://rabble.ca/news/2012/06/remembering-william-commanda-national-aboriginal-day

Condolence from John Duncan, Government of Canada:

http://nouvelles.gc.ca/web/article-eng.do?nid $=614929$

CBC:

http://www.cbc.ca/news/canada/keeper-of-the-wampum-william-commanda-algonquin-elder-1.988042

CTV:

http://ottawa.ctvnews.ca/aboriginal-leader-commanda-dies-at-home-1.678791

Yahoo News:

http://ca.news.yahoo.com/algonquin-elder-william-commanda-dies-174659406.html 
APTN, An Algonquin man of wisdom:

http://aptn.ca/pages/news/2011/08/03/algonquin-elder-commanda-vision-inspiration/

AFN mourn the passing of Algonquin Elder:

http://www.newswire.ca/en/story/821201/afn-and-first-nations-mourn-the-passing-of-algonquin-elder-grandfatherwilliam-commanda-an-inspirational-leader-teacher-and-activist

Indigenous issues:

http://indigenouspeoplesissues.com/index.php?option=com_content\&view=article\&id=11614:ontario-anishinabek-

mourn-passing-of-grandfather-william-commanda\&catid=22:north-america-indigenous-peoples\&Itemid $=55$

Madjashin nin dada:

http://www.blogtopsites.com/outpost/ee0b5a2c216645c831580135b3a1ba90

National Post:

http://news.nationalpost.com/2011/08/03/algonquin-spiritual-leader-dead-at-97/

Ottawa Community News:

http://www.ottawacommunitynews.com/news-story/3796611-algonquin-and-spiritual-elder-dies-at-97/

Media coop, Celebrating Grandfather:

http://www.mediacoop.ca/story/celebrating-grandfather-commanda-morning-star/8220

Commanda passes, The Nation, Eeyou Itchee:

http://www.nationnews.ca/algonquin-elder-commanda-passes-away/

First Nations in BC:

http://fnbc.info/ir-anishinabek-mourn-passing-grandfather-william-commanda

North Bay, Nipissing, First Nations mourn loss:

http://www.northbaynipissing.com/2011/08/first-nations-mourn-loss-of-grandfather-william/

In memoriam, Black Ottawa:

http://blackottawa411.weebly.com/in-memoriam.html

Nigeria villages, Quality of a Welcomne:

http://nigeriavillagesquare.com/pius-adesanmi/elder-commanda-and-the-quality-of-a-welcome.html

Spirit of Change, Grandfather passes:

http://www.spiritofchange.org/editor-s-post/grandfather-william-commanda-passes

Art and Culture News, RIP:

http://apt613.ca/weekly-news-review-rip-william-commanda-30-years-of-the-underpass-and-more/

WCHA, Commanda travels on:

http:// forums.wcha.org/showthread.php?7982-Grandfather-William-Commanda-Travels-On

Keeper of the wampum:

http://article.wn.com/view/2011/08/04/Keeper_of_the_wampum_William_Commanda_Algonquin_elder/\#/related_ne ws

Prophecy of the Seven Fires, Turtle Lodge (pdf):

http://fner.files.wordpress.com/2011/11/theprophesyofthe_27_00.pdf

Sacred Instructions:

http://www.nativevillage.org/Inspiration-/Sacred\%20Instructions.htm

William Commanda on Sacred Places and the Land

http://www.lynngehl.com/2/post/2011/08/post-title-click-and-type-to-edit1.html

First Peoples voices, Native History:

http:// firstpeoplesvoices.com/called.htm

All One Now, interview 2006, chronology: 
http://www.allonenow.org/GrandfatherCommanda.html

Voice of the Taino:

http://uctp.blogspot.ca/2011/08/algonquin-elder-william-commanda-passes.html

Apoyo Bolivia:

http://grupoapoyo.org/basn/node/15752

Windspeaker, footprints:

http://www.ammsa.com/publications/windspeaker/william-commanda-footprints

Bonnechere Algonquin First Nation, Tribute:

http://www.bafn.ca/william.html

Indspire:

https://indspire.ca/laureates/william-commanda/

Organizing for Justice, Tribute:

https://organizingforjustice.ca/?p=623

Earth blog, Grandfather passes:

http://marecromwell.wordpress.com/2011/08/10/grandfather-william-commanda-passes-august-3-2011/

Wawatay News:

http://www.wawataynews.ca/archive/all/2011/8/4/first-nations-grieve-loss-william-commanda_21724

Reflections on the Outdoors Naturally:

http://reflectionsoutdoors.wordpress.com/2011/08/03/algonguin-elder-william-commanda-travels-on/

Newsvine, Commanda passes:

http://kavika.newsvine.com/_news/2011/08/04/7252062-spiritual-leader-and-elder-ojigkwanong-william-commandapasses-on

Native American Prophecy:

http://www.nativeamericanprophecy.com/prophecy-algonquin-william-commanda.htm

Sunray Peace Village:

http://sunraypeacevillage.blogspot.ca/2011/08/william-commanda-spiritual-leader-of.html

Ottawa Riverkeeper:

http://fr.ottawariverkeeper.ca/photos/1_ancien_william_commanda

Warrior Nation:

http://warriornation.ning.com/profiles/blogs/william-commanda-gift-to-the-algonquin-people-passes-into-spirit

Daily Observer, A page of history has closed:

http://www.thedailyobserver.ca/2011/08/04/a-page-of-our-history-has-closed-with-william-commanda

SooToday:

http://www.sootoday.com/content/news/full_story.asp?StoryNumber=53322

William Commanda Travels On:

http://www.network54.com/Forum/352882/thread/1312407907/last-1312421182/William+Commanda+Travels+On

Tribute, Kendal Vale Farm:

http://kendalvale.ca/cheese.html

Biography and facts:

http://www.whoislog.info/profile/william-commanda.html

Sweat: http://www.nowtoronto.com/news/story.cfm?content=173252

Quiz: http://barberouille.webs.com/quizpetittest.htm

Mini-bio: 
http://www.smoeye.com/monitor/586914/facebook/www.facebook.com/pages/William-Commanda/124359520927182

Anishinabek mourn passing of Grandfather (pdf):

http://www.anishinabek.ca/uploads/IRGWCommanda.pdf

Anishinabek mourn passing of Grandfather:

http://www.baytoday.ca/content/news/details.asp?c=43231

CPAWS, William Commanda's legacy:

http://cpaws.org/blog/william-commandas-legacy

The Globe and Mail:

http://www.theglobeandmail.com/news/national/algonquin-elder-william-commanda-was-honoured-for-efforts-to-bridgecultures/article589299/

IPSMO, In Commemoration of Grandfather William Commanda:

http://ipsmo.wordpress.com/2011/08/28/commemoration/

The Call of the Land, RIP Grandfather:

http://thecalloftheland.wordpress.com/2011/08/03/r-i-p-mishomis-grandfather-william-commanda/

Rural American Know-How RIP Grandfather:

www.grit.com/community/r-i-p-grandfather-william-commanda-97.aspx

Indian Time, William Commanda, 'Gift to the Algonquin People,' Passes into Spirit World:

http://www.indiantime.net/story/2011/08/11/reader-submission/william-commanda-gift-to-the-algonquin-peoplepasses-into-spirit-world/2855.html

Indian Country, William Commanda, 'Gift to the Algonquin People,' Passes into Spirit World:

http://indiancountrytodaymedianetwork.com/article/william-commanda- $\% 25 \mathrm{e} 2 \% 2580 \% 2598$ gift-to-the-algonquin-

people $\% 25 \mathrm{e} 2 \% 2580 \% 2599$-passes-into-spirit-world-45654

The Fifth Column, links to articles:

http://the5thc.blogspot.ca/2011/08/his-spirit-will-live-on-grandfather.html

Creating Hope for the Future:

http://hopeforthefuture.ca/2011/blue-post/william-commanda-algonquin-spiritual-leader-dead-97/

Green Living Ottawa, honouring Elder Commanda:

http://greenlivingottawa.com/2011/09/06/honouring-elder-william-commanda/

Masqua's Art, Seven Fires Prophecy:

http://masqua.ca/2013/02/26/william-commanda-and-the-seven-fires-prophesy/

Progressive bloggers:

http://www.progressivebloggers.ca/tag/william-commanda/

ATS, Seven Fires:

http://www.abovetopsecret.com/forum/thread181356/pg4

Short bio:

http://www.evi.com/q/william_commanda_biography

The soul of a Nation has died:

http://www.wabanaki.com/seven_fires_prophecy.htm

Seven Prophets Seven Fires, Wabanaki:

http://www.wabanaki.com/seven_fires_prophecy.htm

William Commanda quotes

7th Fire:

http://learningtogive.org/search/quotes/Display_Quotes.asp?author_id=130\&search_type=author 
Prophétie des 7 Feux, texte et vidéos:

http://www.enigmes.tv/2013/02/22/legendes/la-prophetie-des-7-feux-3283\#more-3283

Manataka, Message from Grandfather 2008:

http://www.manataka.org/page1458.html

Democratic underground, Equinox 2008, Message from Grandfather:

http://www.democraticunderground.com/discuss/duboard.php?az=view_all\&address $=245 \times 76578$

First Peoples worldwide:

http://firstpeoples.org/wp/tag/william-commanda/

Cheyenne gathering:

http://cheyennegathering.yuku.com/topic/1105

Medicine Wheel:

http://www.greatdreams.com/wheels/wheels.htm 